\title{
INVESTIGATION OF CUMULUS CELL FACTORS ASSOCIATED WITH EMBRYO DEVELOPMENT AND PREGNANCY OUTCOME IN HUMAN IVF
}

\section{JOZSEF EKART}

A thesis submitted to the Victoria University of Wellington in fulfilment of the requirements for the degree of Doctor of Philosophy in Cell Biology

Victoria University of Wellington 


\section{ABSTRACT}

Recent evidence suggests that the expression of some candidate genes in cumulus cells (CC) have the potential to serve as markers of oocyte quality. The aims of this study were: 1) to validate a multiplex quantitative polymerase chain reaction (QPCR) method to measure four genes simultaneously in extracts from individual $\mathrm{CC}$ and; 2) to investigate the relationships in individual cumulus-oocyte-complexes (COC) amongst the expression levels of a range of candidate genes $(\mathrm{N}=8)$ from individual $\mathrm{CC}$, the numbers of $\mathrm{CC}$ per $\mathrm{COC}$ and developmental indicators (good blastocyst development and live birth outcome) of the associated oocytes following in vitro fertilization (IVF) with intra-cytoplasmic sperm injection (ICSI).

Sixty eight women were recruited for this study following approval from NZ Multi-Regional Ethics Committee and classified into four research groups: young and healthy women $(<38$ years, $\mathrm{N}=25$ ), young women with polycystic ovarian syndrome ( $<38$ years, $\mathrm{N}=11$ ), young with diminished ovarian reserve ( $<38$ years, $N=12)$ and older and healthy women $(\geq 40$ years, $\mathrm{N}=20$ ). Following exogenous rFSH-assisted ovarian stimulation, $608 \mathrm{COC}$ were collected and subjected to ICSI. Oocyte and embryo quality, and pregnancy outcomes were recorded. Expression levels of the following candidate genes HAS2, FSHR, SLC2A4 (GLUT4), ALCAM, $S F R P 2, V C A N, N R P 1$ and $P R$ in CC extracts from individual COC were measured by TaqMan QPCR and normalized against the house-keeping gene, RPL19. The numbers of CC from individual COC were calculated from RPL19 expression levels plotted against a standard curve of $\mathrm{CC}$ number. These results were then assessed against the outcomes of the associated oocytes following ICSI.

$H A S 2, F S H R, A L C A M, V C A N, N R P 1$ and $P R$ mRNA were detectable in most samples (98.5\%) whereas those for $S L C 2 A 4$ and SFRP2 were generally undetectable. The minimum number of $\mathrm{CC}$ required for QPCR was estimated to be $\sim 70$. The mean levels of FSHR mRNA were up-regulated in young women with PCOS compared to those in the other two groups of women $<38 \mathrm{y}$. Expression levels of HAS2 across all four groups of women were correlated to both biological (age, basal serum FSH and serum AMH) and treatment (amount of rFSH used for stimulation, follicle numbers and $\mathrm{COC}$ retrieved) variables. Investigations related to oocyte development in young and healthy women showed that 1) mean mRNA levels of $V C A N, H A S 2$ and $P R$ were higher $(\mathrm{P}=0.002, \mathrm{P}<0.05, \mathrm{P}<0.05$, respectively) in CC associated with oocytes that resulted in good quality blastocysts and those of VCAN were higher 
$(\mathrm{P}<0.05)$ in $\mathrm{CC}$ associated with oocytes that resulted in a live birth, compared with those with developmental failure. However, the expression levels of all measurable candidate genes were highly variable for individual CC from COC from each woman. Indeed, $99.7 \%$ of individual COC were different in CC mRNA levels and cell composition. The application of a ranking method to score the relative CC mRNA levels of selected candidate genes from each COC recovered from individual women was evaluated. This approach demonstrated a predictive power of $80 \%$ efficiency for selecting good quality oocytes (at least one), whilst requiring the insemination of no more than three oocytes in any treatment cycle. Furthermore, this selection method resulted in a pregnancy and live birth rate of 60 and 52\% respectively ( $\mathrm{N}=25$ women). This outcome is similar to that achieved when all metaphase II (MII) oocytes are inseminated.

In conclusion, this study has validated a multiplex QPCR method to quantify the expression levels of four genes in $\mathrm{CC}$ of individual human COC simultaneously using as few as 70 cells. Moreover, that there is sufficient cDNA so that many more candidate genes can be measured in the same extract. From the knowledge of the mRNA levels of at least four genes, VCAN, $F S H R, H A S 2$ and $P R$ in $C C$, it is possible to improve upon existing biological indicators the potential to predict good blastocyst formation and a successful pregnancy outcome. It is concluded that the application of a multiplex QPCR approach to assess the expression levels of a limited number of marker genes in CC can be used to select the best oocytes for successful pregnancy outcomes. 


\section{ACKNOWLEDGEMENTS}

First and foremost, I would like to thank those women who consented to this study. Further, I would like to aknowledge the guidance and help of several individuals who generously and willingly contributed their expertise and knowledge for the completion of this thesis.

I offer my sincerest gratitude to my supervisors, Prof. Ken McNatty from Victoria University of Wellington, and Prof. John Hutton from University of Otago, Wellington. Words cannot describe how privileged and honoured I feel for having had the opportunity to work with such fantastic individuals. I would like to thank Ken for believing in me, encouraging me throughout the years, for his infinite knowledge, and for his kindness and patience whilst allowing me the room to work in my own way. I would like to thank John for believing in me since the very beginning, for his tremendous help in managing the women recruited for this study, for his friendship, and last but not least, for his sense of humour.

Dr Janet Pitman, my secondary supervisor from Victoria University of Wellington, for her invaluable expertise in the validation of the multiplex QPCR methodology and in the preparation of manuscripts. I would further like to thank her for her patience and unfailing support throughout the completion of this dissertation.

Dr Kevin Coetzee, my laboratory supervisor from Fertility Associates, for his help, encouragement and his great friendship throughout the study.

Fertility Associates Ltd, for their financial and strategic support, without which this study would not have been possible. I would also like to thank my embryologist colleagues from Fertility Associates Wellington and my colleagues from the Reproduction Team from Victoria University (especially Adrian Bibby) for their time, support and knowledge.

Last but not least, I would like to thank to my family, my girlfriend and my fellow students (especially Shalen) for their great friendship and great memories spent together. It has been a very rewarding opportunity to study at Victoria University of Wellington, an experience for which I will be eternally grateful. 


\section{PRESENTATIONS}

\section{2}

The Fertility Society of Australia $31^{\text {st }}$ Annual Scientific Meeting, Auckland, New Zealand Title: "Cumulus cell mRNA markers for identifying healthy oocytes"

American Society of Reproductive Medicine (ASRM) $68^{\text {th }}$ Annual Meeting, San Diego, USA Title: "The use of cumulus cell (CC) mRNA levels to predict blastocyst development and live birth outcomes in women undergoing intracytoplasmic sperm injection (ICSI) and single embryo transfer"

Controversies in Reproductive Medicine, Taupo, New Zealand

Title: "Cracking Good Eggs - An "in-gene-ious" method! Oocyte quality and the use of cumulus cell markers for the selection of oocytes with developmental potential in young and healthy, POF, PCOS and older women undergoing ICSI"

\section{1}

IVF Scientists Meeting, Auckland, New Zealand

Title: "The use of cumulus cells to predict embryological and pregnancy outcomes in women undergoing IVF"

\section{0}

The Fertility Society of Australia $29^{\text {th }}$ Annual Scientific Meeting, Adelaide, Australia Title: "Can cumulus cell gene expressions predict embryology outcome?"

\section{AWARDS}

\section{2}

ASRM CMC In-Training Travel Award, $68^{\text {th }}$ Annual Meeting of ASRM, San Diego, USA

\section{1}

Obex Award for Best Paper, IVF Scientists Meeting, Auckland, New Zealand 


\section{TABLE OF CONTENTS}

Content

Page number

ABSTRACT

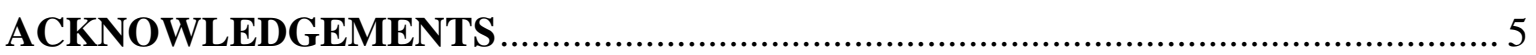

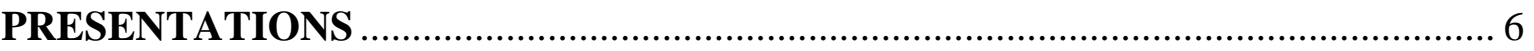

AWARDS

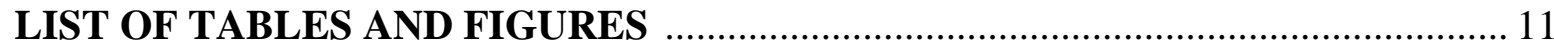

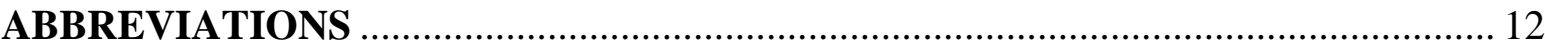

\section{Chapter 1 INTRODUCTION}

1.1 Short history of ART and the development of human IVF ................... 15

1.2 The origin of the female gametes ...................................................... 16

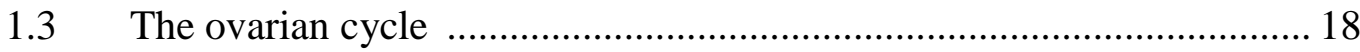

The hormonal regulation of folliculogenesis ...................................... 23

1.4 Ovarian stimulation in human IVF ................................................... 24

1.5 The oocyte's central role in follicular development ............................. 27

The role of oocyte secreted factors $(\mathrm{OSF})$............................................. 27

1.6 The outcome of human oocytes following oocyte collection: IVF,

pre-implantation genetic diagnosis and fertility preservation ................ 31

IVF treatment and associated therapies for increasing

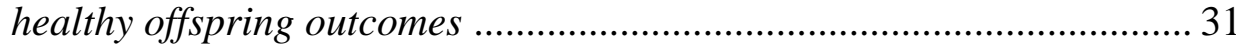

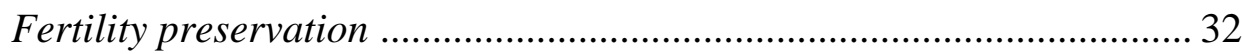

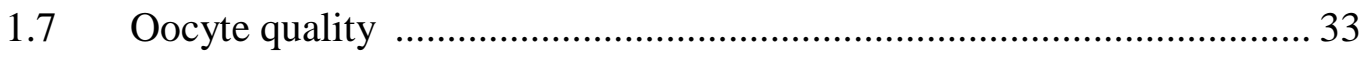

Factors associated with oocyte quality ................................................ 33

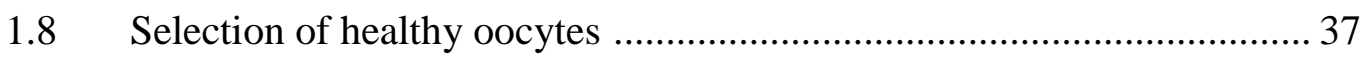

Oocyte selection according to morphological characteristics ................ 38

Oocyte selection according to COC morphology ....................................39

Oocyte selection according to cumulus cell gene expression ................. 39

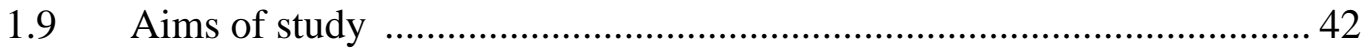




\section{Chapter 2 METHODOLOGY}

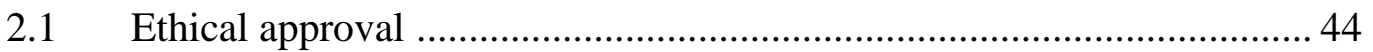

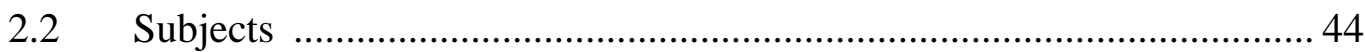

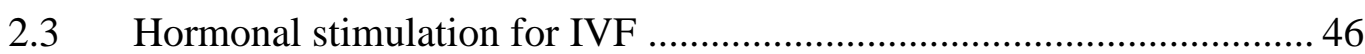

2.4 Embryological manipulation and CC collection .................................. 47

2.5 Pregnancy monitoring and live birth follow-up .................................. 50

2.6 Cell count and viability index for cumulus cells after OPU ..................50

2.7 RNA Extraction and cDNA Synthesis ............................................... 51

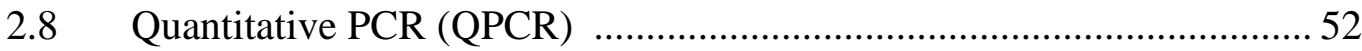

2.8.1 SYBR Green Method .......................................................................... 52

2.8.2 Multiplex (TaqMan) Method ............................................................ 53

2.8.3 Fluorescence Monitoring in Real-Time .......................................... 53

2.8.4 Primers and Probes ............................................................................. 54

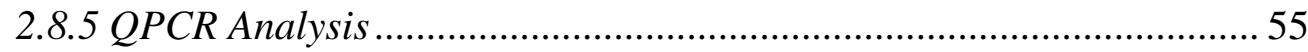

2.8.6 Confirmation of genes by DNA sequencing ...................................... 56

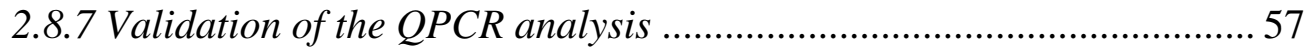

2.8.8 Quantification of gene expression by multiplex QPCR ...................... 58

\section{Chapter 3 VALIDATION OF RPL19 AS A HOUSEKEEPING GENE FOR HUMAN}

\section{CUMULUS CELLS}

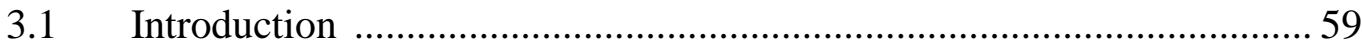

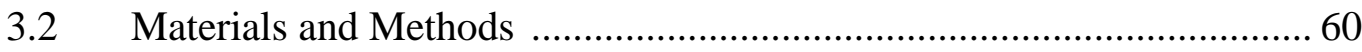

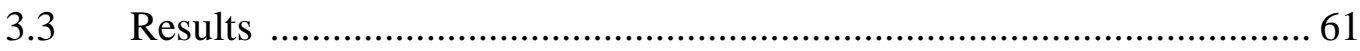

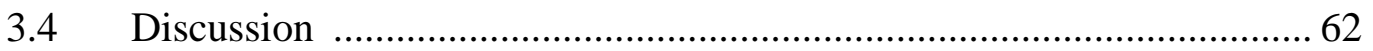

\section{Chapter 4 OOCYTE DEVELOPMENTAL INDICATORS AND RELATIONSHIPS} WITH CC NUMBERS

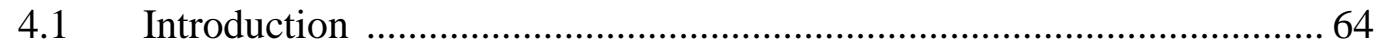

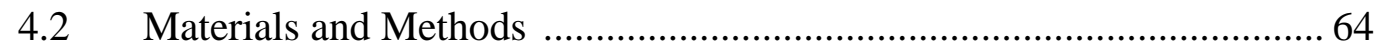

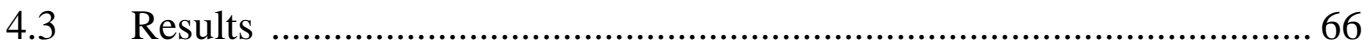

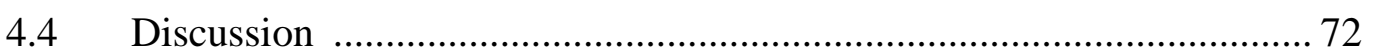


Chapter 5 EFFECT OF AGE, INFERTILITY BACKGROUND AND FSH

TREATMENT ON HAS2, FSHR, SLC2A4 (GLUT4) mRNA LEVELS IN

\section{CUMULUS CELLS}

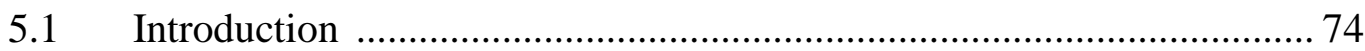

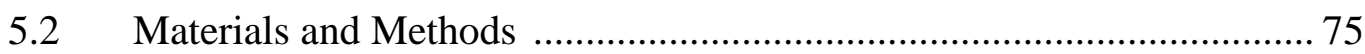

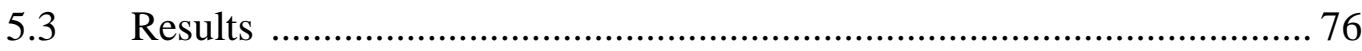

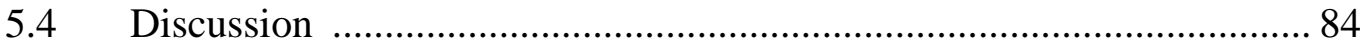

Chapter 6 MESSENGER RNA LEVELS IN CUMULUS CELLS: RELATIONSHIPS TO EMBRYOLOGICAL AND PREGNANCY OUTCOMES

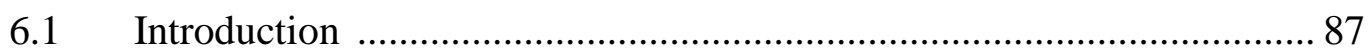

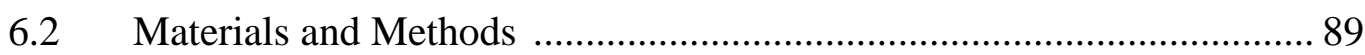

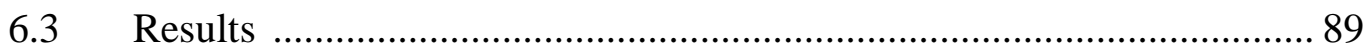

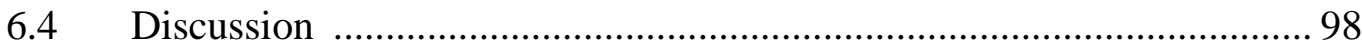

Chapter 7 RANKING OF INDIVIDUAL COC ACCORDING TO CC

GENE EXPRESSION LEVELS AND CC NUMBERS AND THE

SELECTION OF OOCYTES WITH HIGH DEVELOPMENTAL

POTENTIAL

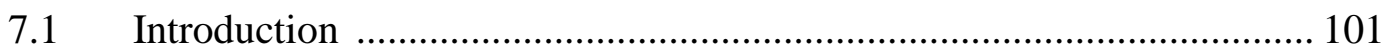

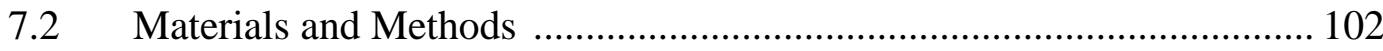

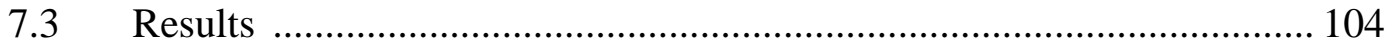

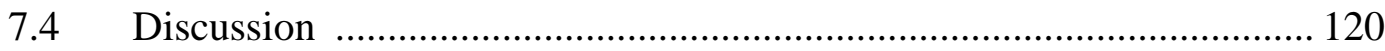

\section{Chapter 8 DISCUSSION}

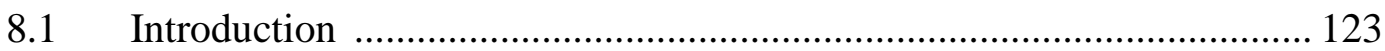

8.2 Validation of a multiplex QPCR method ............................................ 123

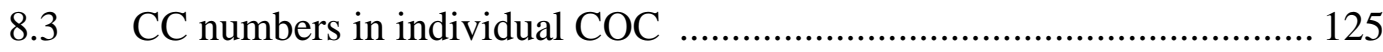

8.4 CC mRNA levels in individual COC ............................................... 125

$C C m R N A$ levels are affected by the endocrine status of the individual as well as by the exogenous rFSH treatment ........................ 126

CC mRNA levels are predictive of oocyte maturity .............................. 127

CC mRNA levels are predictive of blastocyst development and

live birth outcome 
Candidate genes recommended for future investigations 128

Candidate genes with poor potential for oocyte selection

8.5 The ranking of individual MII oocytes according to CC mRNA levels, and the application of this ranking system for oocyte selection

8.6 Investigations regarding the developmental synchrony of individual COC based on CC mRNA levels and CC numbers

8.7 Clinical implications of the findings presented in this study ................. 134

8.8 Conclusions

\section{APPENDICES}

Appendix 1 Patient Consent Form 137

Appendix 2.1 Long course stimulation with pill for IVF, protocol 5.85 138

Appendix 2.2 Microdose flare stimulation for IVF, protocol 5.9 142

Appendix 3.1 ICSI Protocol 145

Appendix 3.2 Fertilisation check 149

Appendix 3.3 Embryo grading and selection 151

Appendix 4 Tables for recording COC and oocyte morphological features 154

Appendix 5 Table 5C 155

Appendix 6 Figure $6 \mathrm{I}$ 156

Appendix 7 Figure 7C (full data set). 158 


\section{LIST OF TABLES AND FIGURES}

Tables

Page number

$2 \mathrm{~A}$ 54

$2 \mathrm{~B}$ 55

$2 \mathrm{C}$ 57

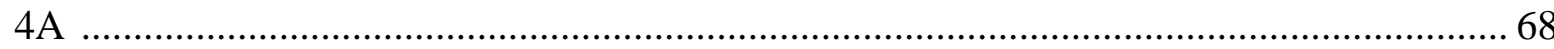

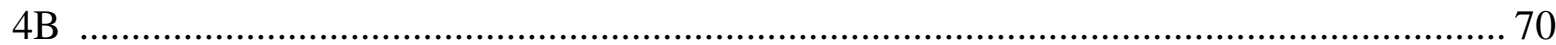

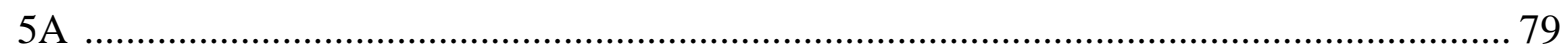

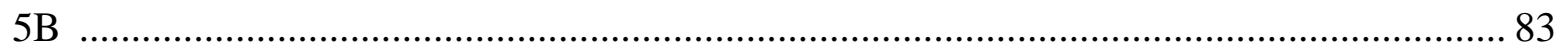

7 116

Figures

Page number

$1 \mathrm{~A}$

$1 \mathrm{~B}$

$1 \mathrm{C}$

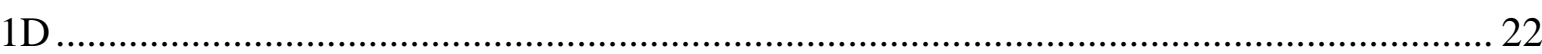

$1 \mathrm{E}$

3

5

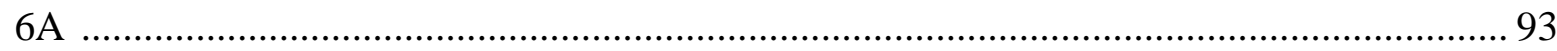

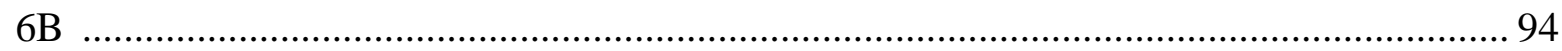

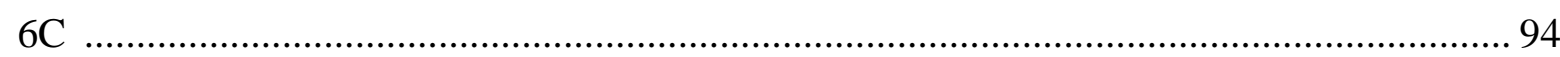

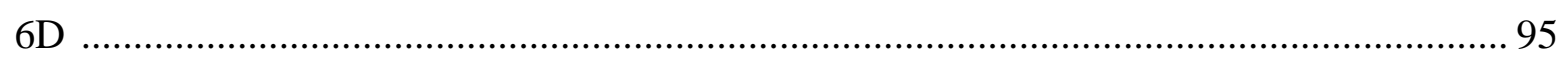

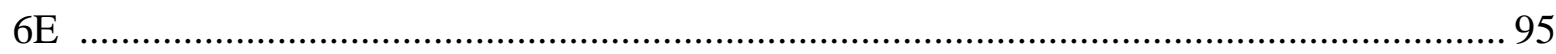

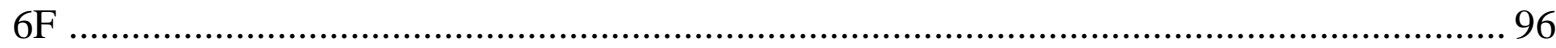

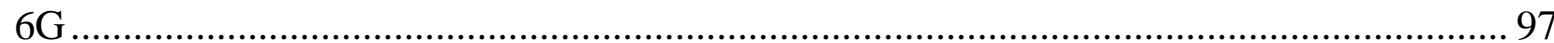

$6 \mathrm{H}$

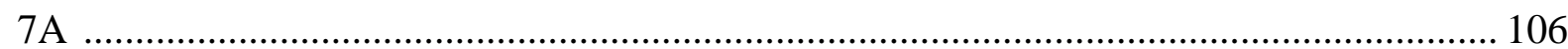

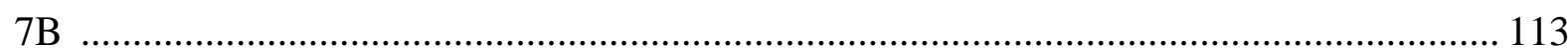

$7 \mathrm{C}$

7D 


\section{ABBREVIATIONS}

BMP6

BMP15

$\mathrm{CC}$

CL

$\mathrm{COC}$

CPAC

$\mathrm{C}_{\mathrm{T}}$

DET

EGF

FA

FAW

FGF

FHB

FSH

FSHR

GAPDH

GC

GDF9

GLUT4 (SLC2A4)

GnRH

$\mathrm{Gp}$

GREM1

GV

HAS2

hCG

HMG

ICM

ICSI

IVF

IVM

KL

LB
Bone morphogenetic protein 6

Bone morphogenetic protein 15

Cumulus cell

Corpus luteum

Cumulus-oocyte-complex

Clinical priority access criteria

Cycle Threshold

Double embryo transfer

Epidermal growth factor

Fertility Associates Ltd.

Fertility Associates Wellington

Fibroblast growth factor

Foetal heart beat

Follicle stimulating hormone

Follicle stimulating hormone receptor

Glyceraldehyde-3-phosphate dehydrogenase

Granulosa cell

Growth differentiation factor 9

Solute carrier 2, member 4

Gonadotrophin-releasing hormone

Group

Gremlin 1

Germinal vesicle

Hyaluronan synthase 2

Human chorionic gonadotrophin

Human menopausal gonadotrophin

Inner cell mass

Intra-cytoplasmic sperm injection

In vitro fertilization

In vitro maturation

Kit ligand

Live birth 
Metaphase I

MII

Metaphase II

NO

Negative outcome

NRP1

Neuropilin 1

$\mathrm{OCP}$

Oral contraceptive pill

OSF

Oocyte secreted factor

PCA

Principal component analysis

PCOS

Polycystic ovarian syndrome

PGD

Preimplantation genetic diagnosis

PR

Progesterone receptor

PTGS1

Prostaglandin-endoperoxide synthase 1

PTGS2

Prostaglandin-endoperoxide synthase 2

PTX3

Pentraxin 3

QPCR

Quantitative PCR

$\mathrm{rFSH}$

Recombinant FSH

RPL19

60S ribosomal protein L19

SET

Single embryo transfer

SFRP2

Secreted frizzled-related protein 2

SLC38A3

Solute carrier family 38

TCA

Tricarboxylic acid

TGF- $\beta$

Transforming growth factor- $\beta$

TNFAIP6

Tumour necrosis factor $\alpha$-induced protein 6

VCAN

Versican

$\mathrm{ZP}$

Zona pellucida 


\section{Chapter 1 INTRODUCTION}

\subsection{Short history of ART and the development of human IVF}

The process of reproduction has long fascinated mankind from both philosophical and scientific perspectives. The discovery of reproductive gametes was associated with the development of microscopy as an investigative tool. The first observation of the human male gamete (spermatozoa) dates back to 1677, when Antonj van Leeuwenhoek conveyed his observations in a letter to the Royal Society, published in the Philosophical Transactions in 1679. At that time Leeuwenhoek described the spermatozoa as 'animalcules' or 'spermatic worms'. On the other hand, female gametes were discovered much later. Although Niels Stensen in 1667 proposed that the 'female testes' contained oocytes, it wasn't until 1826, when Carl Ernst von Baer identified first the mammalian oocyte during his studies on the ovary of a dog. The first in vitro fertilization (IVF) attempt dates back to 1770, when Lazaro Spallanzani experimented with frogs, and discovered that oocytes would only develop in tadpoles after contact with semen (Clarke 2006). Later in the early nineteenth century, JeanLouis Prevost and Jean-Baptiste Dumas demonstrated that one spermatozoon can fertilize only one egg (Clarke 2006). The first report on human IVF was by Robert Edwards and colleagues in 1969, when they observed pronuclear formation following fertilization of oocytes (Edwards et al. 1969). In 1970, these researchers reported that they had obtained embryonic development to the 16-cell stage (Edwards et al. 1970). In 1973, a team from Monash University, Australia reported the first IVF pregnancy (De Kretzer et al. 1973). However, it wasn't until 1978 when the first live birth resulting from IVF was reported by Steptoe and Edwards, following the birth of Louise Brown on $25^{\text {th }}$ July 1978 (Steptoe and Edwards 1978; Clarke 2006). On $4^{\text {th }}$ October 2010, the Nobel Prize in Physiology or Medicine was awarded to Robert Edwards for his pioneering work on IVF (Kirby 2010; Watts 2010).

Human IVF can be described as a medical laboratory technique by which oocytes (eggs) are fertilized by sperm under artificial conditions, outside the body. A human IVF procedure involves the following stepwise events: ovarian stimulation, oocyte retrieval, oocyte fertilization, embryo transfer and the support of implantation and pregnancy (Smulders et al. 2010). IVF has been developed and improved upon over many years of research with the intention of helping infertile couples to have a healthy offspring and establish a family. 


\subsection{The origin of the female gametes}

The formation of female gametes is associated with the development of the ovaries in the foetus. The ovary is a multi-compartmental organ with two key functions: 1) generation of fertilizable, developmentally competent female gametes (oocytes) and; 2) the secretion of steroid hormones required for preparation of the reproductive tract for fertilization of the oocyte and subsequent establishment of pregnancy (Knight and Glister 2006). Around the fifth month of gestation in humans, the ovaries contain peak numbers (ca. 6-7 million) of female germ cells (i.e. oogonia) (Baker 1963), the precursor cells for the development of oocytes. At this stage of gestation, oogonia enter meiosis which is the process by which a single oocyte and the polar bodies form (Ohno et al. 1962). During the transition through meiosis to prophase, most (>70\%) germ cells die by apoptosis, leaving between 1-2 million oocytes at the prophase stage in the first meiotic division. These surviving oocytes with an associated layer of somatic cells known as granulosa cells constitute what is often referred to as the primordial follicle pool or "ovarian reserve" (see reviews by van Wagenen and Simpson 1965; Baker 1972; Rabinovici and Jaffe 1990; McNatty et al. 2000). As primordial follicles form during foetal life, they start to leave the primordial pool asynchronously and begin to grow towards ovulation (Peters et al. 1975; Byskov et al. 2005). Due to inadequate hormonal support before puberty, the fate of these growing follicles is atresia, resulting in a decline in follicles numbers to approximately 3-400,000 at puberty. Thereafter during the reproductive life in women, only about 400 will eventually complete development, and lead to oocytes being released at ovulation. At the time of menopause, $\leq 1000$ oocytes remain (Baker 1963; Peters 1976; Maltaris et al. 2009). Nevertheless, from the time follicles form in foetal life, the initiation of growth of follicles from the primordial pool towards ovulation is a process that continues throughout life, regardless of reproductive state (i.e. puberty, pregnancy etc.) until the pool becomes exhausted around the time of menopause (Peters et al. 1975).

As discussed, oogonia that enter meiotic prophase are termed oocytes. During their development, oocytes undergo two meiotic cell divisions. Each meiotic division is divided into four phases: prophase, metaphase, anaphase and telophase. The first meiotic prophase in oocytes is unique in that it lasts much longer compared to that in any other cell. Whilst prophase is completed within hours in any other cell, it lasts for weeks, months or years in the oocyte. The various stages of meiotic prophase are defined by the homologous recombination 
of the DNA and the distinct arrangement of the chromosomes, and therefore are morphologically distinguishable. They can be divided into the transitory phase (leptotene, zygotene and pachytene) and the stationary phase (diplotene). The stages are of different duration and become progressively longer as prophase progresses: leptotene is shortest and diplotene the longest. As the cells enter diplotene they begin the stationary phase in which the oocytes remain until shortly before ovulation. The first meiotic division is reductional, i.e. the diploid ( $2 \mathrm{~N}, 46$ chromosomes or 23 pairs of chromosomes) primary oocyte divides into two haploid (N, 23 chromosomes, each consisting two sister chromatids) cells, namely the secondary oocyte and the first polar body. The secondary oocyte begins the second meiotic division but is arrested during a stage called metaphase II. The process by which the oocyte is released from the ovary is called ovulation. If fertilization occurs, the secondary oocyte completes the second meiotic division by halving its genetic material to 23 chromosomes (single chromatids) and releasing the second polar body containing the other pair of the 23 chromosomes. Then the 23 chromosomes from the nucleus of the spermatozoa will join the 23 chromosomes of the oocyte to form a zygote and complete fertilization (Figure 1A) (Ohno et al. 1962; Peters and McNatty 1980). 


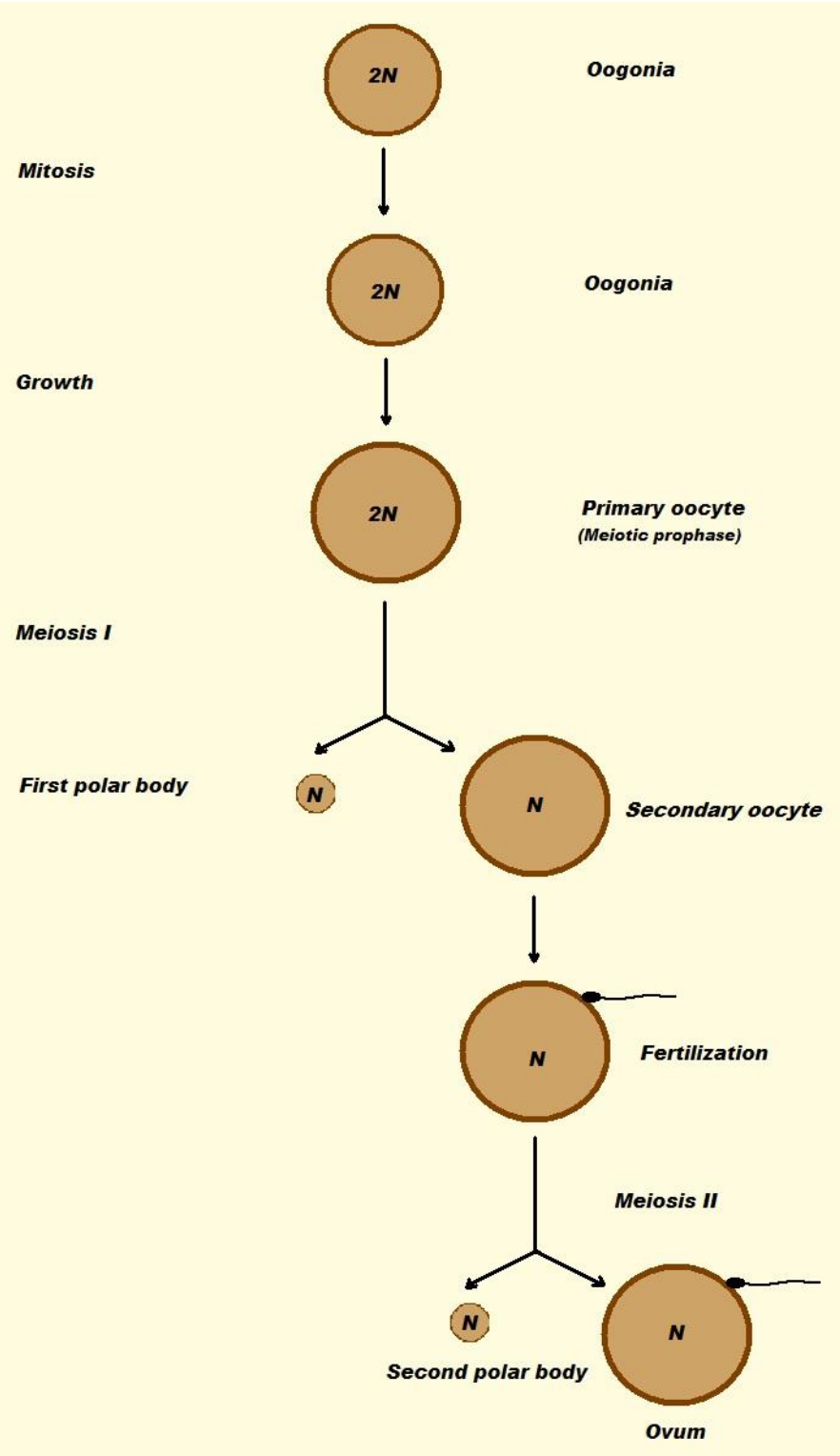

Figure 1A: Diagram showing the process of oogenesis in mammals and humans. $\mathrm{N}=$ number of chromosomes (i.e. 23 chromosomes in humans).

\subsection{The ovarian cycle}

The ovarian cycle is a term used to describe the cyclical functions of the ovary during sequential ovulatory intervals under the control of the hypothalamic-pituitary axis. In women, this first begins at puberty (11-13 years) and ends at menopause (45-55 years). Normally, each cycle lasts 28 days and is divided in two phases of similar duration. The follicular (or proliferative) phase begins on the first day of menses and ends with ovulation. The luteal phase (or secretory phase) is the latter phase of the menstrual cycle and begins after ovulation 
with the formation of the corpus luteum and ends with either a pregnancy or luteolysis and the initiation of a follicular phase (Chabbert Buffet et al. 1998). Most follicles within the ovary are present as primordial structures (about 30-60 $\mu \mathrm{m}$ in diameter) consisting of a primary oocyte encompassed by a single layer of flattened granulosa cells, and separated from the stromal tissue by the basal lamina membrane (Oktem and Oktay 2008). Whilst the level of functional activity in primordial follicles is low, over 500 genes related to housekeeping functions and DNA repair are activated (McNatty et al. 2006; Gougeon 2010). Nevertheless these follicles can sense their microenvironment as they express numerous receptors for growth factors and some components of post-receptor signalling pathways (Eppig 2001; Skinner 2005; McNatty et al. 2006; Gougeon 2010). The process of activation of primordial follicles for growth still remains to be elucidated. However, there is compelling evidence that the signalling between the oocyte and surrounding granulosa cells and interstitium are important for normal follicular recruitment and development (Knight and Glister 2006; Franks et al. 2008). Primordial follicles that have initiated growth undergo a sequential series of morphological changes. During the first step, the oocyte begins to enlarge in diameter, and the surrounding granulosa cells become cuboidal in shape and thereafter increase in number to form a primary follicle (Gougeon and Chainy 1987; Lundy et al. 1999; Braw-Tal 2002). During this time, the oocyte genome is further activated to include rudimentary paracrine signalling pathways that are vital for communication between the follicular somatic cells and the oocyte (McNatty et al. 2006; Gougeon 2010). Whilst these primary follicles develop receptors to follicle stimulating hormone (FSH), they remain essentially gonadotrophin-independent until antrum formation. After the primary stage of growth, a glycoprotein matrix called the zona pellucida (ZP) forms around the oocyte, separating it from the granulosa cells. Granulosa cells continue to proliferate further with the formation of several concentric layers around the primary oocyte (Knight and Glister 2006). As soon as the oocyte is surrounded by more than one-two layers of granulosa cells, the follicle is commonly referred to as a secondary follicle (Eppig 2001). The diameter of a follicle increases from $40-60 \mu \mathrm{m}$ at the primary stage to $120-150 \mu \mathrm{m}$ at the late pre-antral stage (Gougeon and Chainy 1987; Gougeon 1989). New evidence supports the theory that the transition from primary to secondary stage and the growth to the preantral/early antral follicle stage is regulated by local factors, most notably by members of the transforming growth factor- $\beta$ (TGF- $\beta$ ) super-family. As the multiple concentric layers of granulosa cells develop, fluid-filled spaces form amongst the granulosa cells, and stromal cells immediately outside the basal lamina differentiate into the theca layer around the follicle (Knight and Glister 
2006). This thecal layer is intermingled amongst a blood capillary network that increases in prominence as follicular growth continues. With increasing growth, the fluid-filled spaces amongst the granulosa cells coalesce to form an antrum thereby displacing the oocyte to a more eccentric location. The newly-formed antral follicle is marked by a relatively large oocyte around $85 \%$ of its final diameter and surrounded by the ZP, approximately 8-9 layers of FSH-responsive GC, a basal lamina, an LH-responsive theca interna, a capillary network, and a theca externa (Figure 1B).

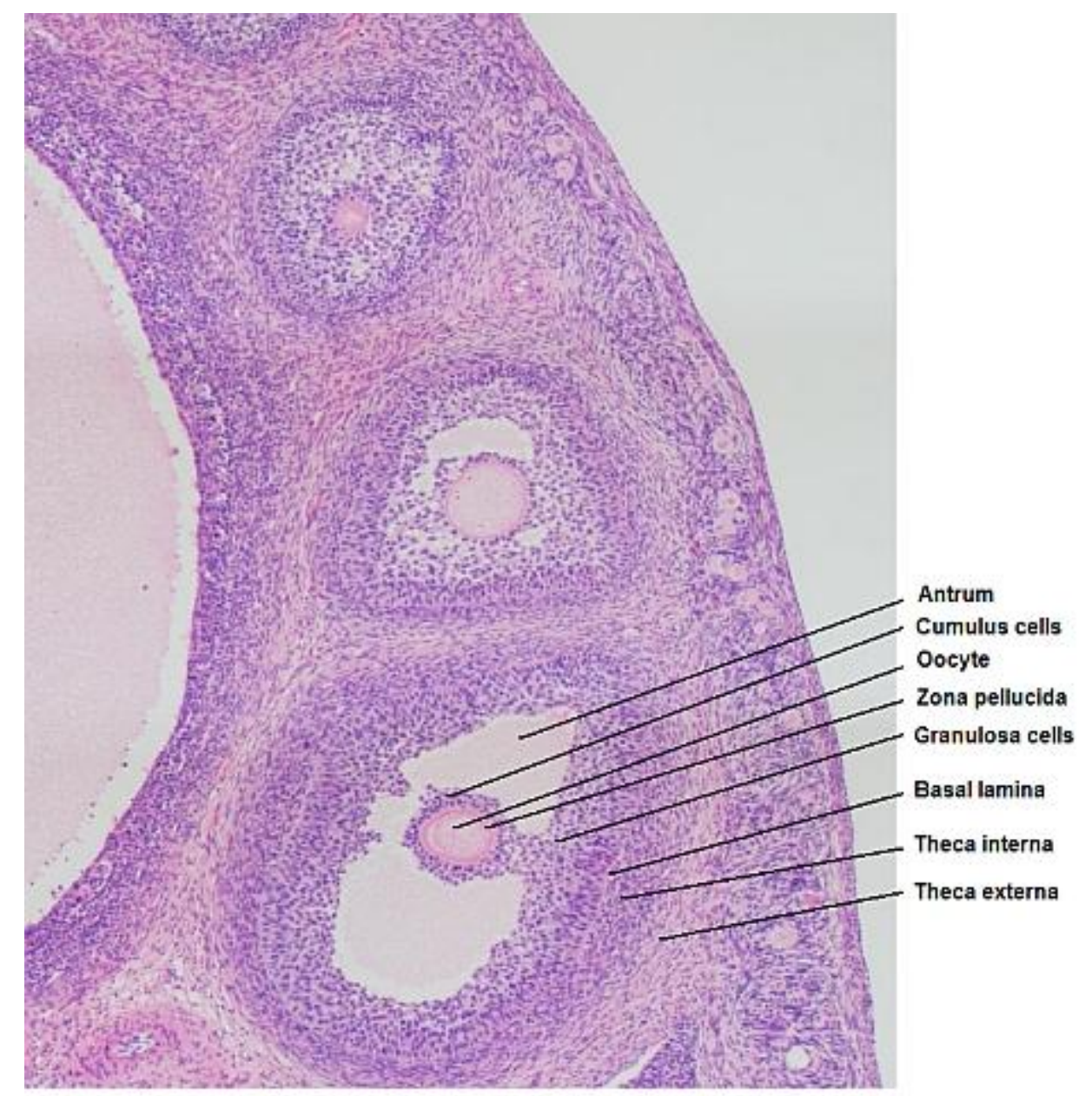

Figure 1B: Section through ovary of a sheep showing three antral follicles of different sizes. Image supplied by Prof Ken McNatty.

Growth beyond the small-antral stages of development becomes critically dependent on the gonadotrophins, FSH and LH with an increase in the rate of follicular growth (Gougeon 1998). Under the control of the oocyte-secreted factors, the GCs immediately adjacent to the oocyte differentiate into cumulus cells (CC), and develop a different functional phenotype 
compared to the more distantly-located (mural) granulosa cells (GC) that will line the basement membrane (Li et al. 2000). Differences in gene expression between the CC and GC populations likely reflect their different roles during follicular maturation (Gilchrist et al. 2008). The CC are clustered in multiple layers around the oocyte (see Figure 1C). Their major role is to support oocyte development with an essential role in nurturing oocyte growth and metabolism by providing nutrients such as amino acids and substrates for energy production (Sugiura et al. 2005). In contrast, the murally-located GC play a major role in follicular steroidogenesis by co-operating with the theca interna to secrete oestradiol (Armstrong and Dorrington 1977; McNatty et al. 1981a). Follicular growth after the antral stage is characterized by further proliferation of GC and theca cells, increased vascularisation around the theca interna and oocyte growth (Oktem and Urman 2010). In women, the development of large antral follicles from $\sim 4 \mathrm{~mm}$ diameter is critically dependent on FSH support together with, as yet, an undetermined level of oocyte growth factor support. Although each small antral follicle has the potential to reach full maturation, only those with GC having the greatest sensitivity to FSH will develop further (McNatty et al. 1981b). As the FSH concentrations gradually decline during the follicular phase, a single large follicle ( $>8 \mathrm{~mm}$ diameter) under the control of pulsatile $\mathrm{LH}$ secretion will gain dominance and reach a diameter of $15-25 \mathrm{~mm}$, and thereafter ovulate in response to the preovulatory LH surge (Figure 1D) (Fauser and Devroey 2003; Palermo 2007).

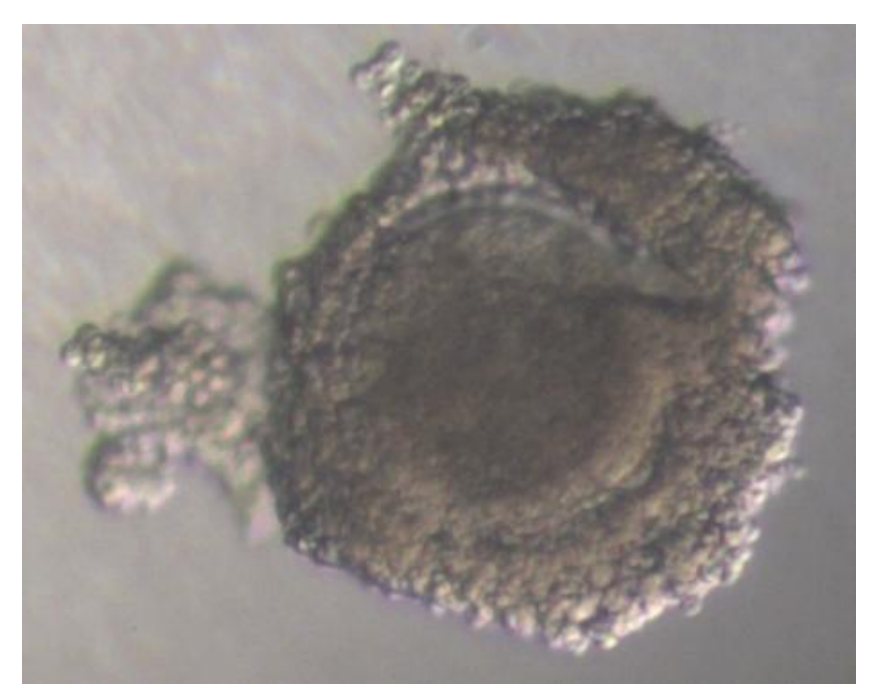

Figure 1C: High resolution image (200 x magnification) showing a sheep COC. The oocyte in the middle is surrounded by multiple layers of CC. Image supplied by Dr Janet Pitman. 


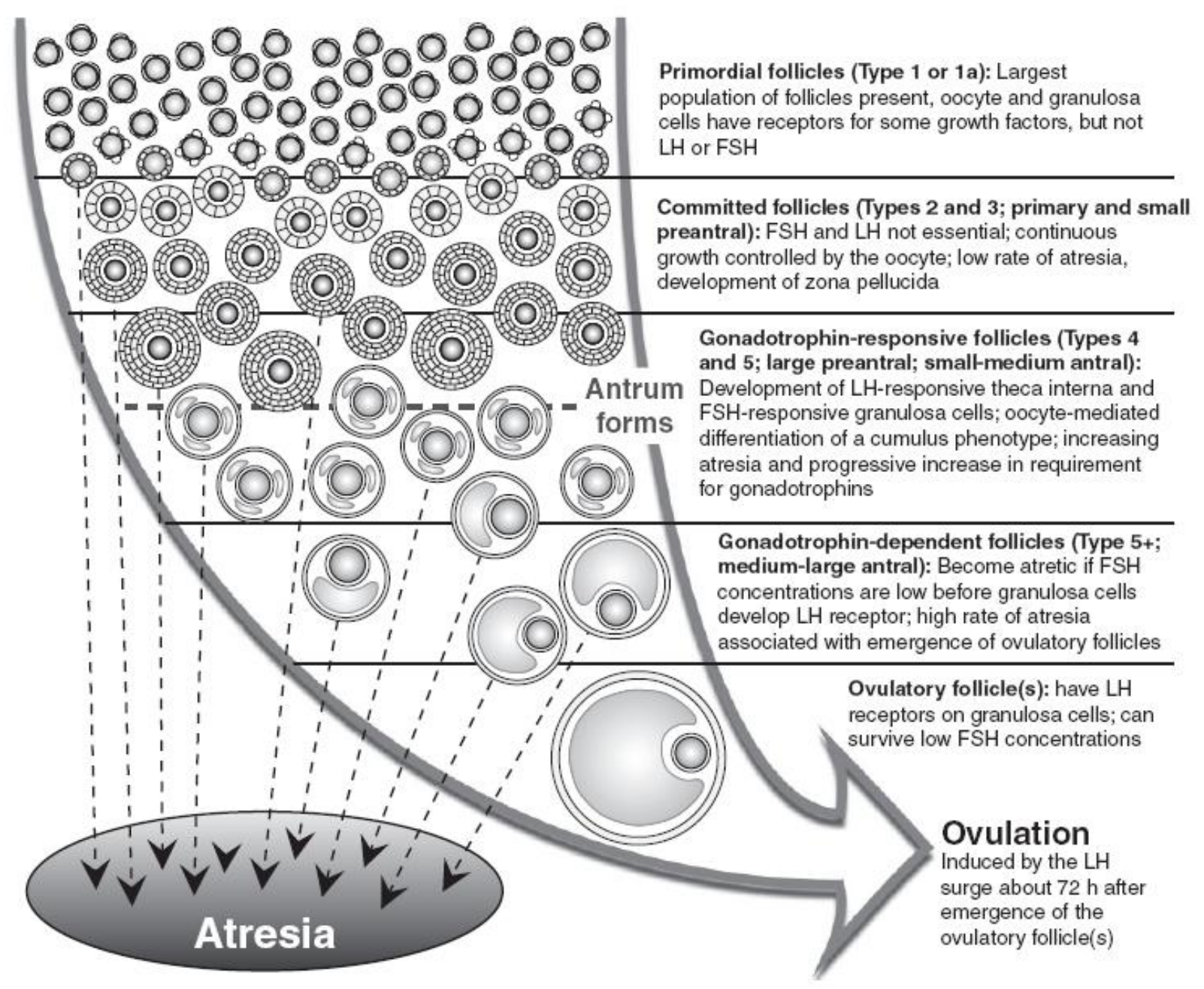

Figure 1D: Graphical representation of the process of folliculogenesis in the ewe developed by Scaramuzzi et al., 1993, 2011. The initiation of follicular growth from the pool of primordial follicles takes place in a continuous and asynchronous manner. Those follicles that start to grow, continue to grow without rest until they become atretic or ovulate. Permission to use this figure in the present thesis was granted by the authors and by CSIRO PUBLISHING (link to original paper: http://www.publish.csiro.au/nid/44/paper/RD09161.htm).

During ovulation, the mature follicle ruptures and the follicular fluid containing the oocyte and associated CC is released onto the ovarian surface from where it is swept into the fallopian tube by the fimbriae (Kolle et al. 2010). Once the egg is within the fallopian tubes, it migrates to the segment known as the ampulla, where the oocyte may be fertilized (Devoto et al. 2009). Thereafter, the fertilized egg (i.e. embryo) continues to travel towards the uterus. After approximately 5 days, the embryo reaches the uterine cavity, and about a day later, 
begins to implant in the anterior wall of the uterus, known as the fundus. It is at this site that the embryo it will continue to grow and develop (Croxatto 2002).

The preovulatory LH surge, which is responsible for the induction of ovulation, also results in the ruptured follicle undergoing a transformation to form a highly vascularised corpus luteum (CL). The CL is composed of luteal cells (formed from theca and granulosa) and nonsteroidogenic (endothelial, immune and fibroblast) cells. The major role of the CL in women is to secrete the steroid hormones (progesterone, androgens and oestradiol), which play a critical role in establishing endometrial receptivity for successful implantation and are essential for the maintenance of early pregnancy. If implantation takes place, the trophoblastic cells of the embryo secrete human chorionic gonadotrophin (hCG) that prevents the regression of the CL. Thereafter, the CL enlarges and remains capable of producing steroids throughout most of the pregnancy. On the other hand, if pregnancy does not occur, the CL undergoes a process of regression, known as luteolysis: this process results in the loss of both functional and structural integrity of the gland, shrinkage and eventually its disappearance. And as a consequence, a new menstrual cycle begins (Devoto et al. 2009).

\section{The hormonal regulation of the folliculogenesis}

Folliculogenesis during the menstrual cycle is regulated mainly by the endocrine system. In 1977 Andrew Schally shared the Nobel Prize with Roger Guillemin for the "discoveries concerning the peptide hormone production of the brain" for describing the neurohormone GnRH (Hodgen 1991). At the beginning of a new follicular phase, there is an increase in the frequency of pulsatile secretion of gonadotrophin-releasing hormone $(\mathrm{GnRH})$ from the hypothalamus. Thereafter, it binds to specific GnRH receptors on gonadotrophic cells in the anterior pituitary gland where it stimulates the synthesis, secretion and release of the gonadotrophins, FSH and LH. In turn, these two hormones stimulate the growth and maturation of antral follicle(s) to ovulation (Huirne et al. 2004).

FSH is a complex heterodimeric glycoprotein produced in the anterior lobe of the pituitary gland. The role of FSH during the antral stages of follicular development is critical (Huirne et al. 2004). Only follicles that respond, and gain increased sensitivity, to FSH will enter the final stages of follicular development and ovulate (Ross et al. 1970; Brown 1978; Macklon and Fauser 1998). Each growing follicle possesses a threshold requirement for stimulation by 
circulating FSH. This threshold needs to be passed to ensure ongoing follicular development. During a typical menstrual cycle, normally only one follicle will become responsive to FSH above this threshold (Brown 1978; McNatty et al. 1983). Other less developed antral follicles during this growth phase undergo atresia as the FSH levels start to fall due to rising oestradiol secretion from the most developed follicle (Huirne et al. 2004).

LH is also a heterodimeric glycoprotein secreted from the anterior pituitary gland. LH is the most critical hormone with respect to the timing of ovulation and the production of steroid hormones. The LH-stimulated androgen synthesis by the thecal cells, and the acquisition of $\mathrm{LH}$ receptors on the GC results in the aromatization of androgen to oestradiol and increasing amounts of oestradiol being produced in and secreted from the dominant follicle, that in turn triggers the preovulatory GnRH and LH surge. As a result, the oocyte resumes meiotic maturation and the dominant follicle ruptures to release the oocyte into the fallopian tube (Huirne et al. 2004).

\subsection{Ovarian stimulation in human IVF}

The first attempts at in vitro fertilization (IVF) therapies in the 1970s involved ovarian stimulation using human menopausal gonadotrophin (hMG). However this approach did not lead to successful pregnancies, presumably due to abnormal luteal function. These stimulation protocols were discarded temporarily, and new approaches were commenced through natural cycles. (Fauser and Devroey 2003) which resulted in the first successful IVF baby (Steptoe and Edwards 1978). Following this, pregnancies were reported using antioestrogen clomiphene citrate, hMG, urinary FSH and later recombinant FSH (rFSH) (Fauser and Devroey 2003). Over the years, the hormonal stimulation protocols have become more sophisticated, and by 1985, stimulated IVF cycles resulted in much higher success rates than for natural IVF cycles (Fishel et al. 1985). A recent review compared the success rates for natural IVF cycles with stimulated IVF cycles and reported that stimulated IVF cycles result in significantly higher pregnancy rates compared with natural IVF cycles in both young $(<35$ years of age) and older (35-38 years of age) women: the pregnancy rate per treatment initiated for young women was $45.4 \%$ in stimulated compared with $15 \%$ in natural cycles and for older women, it was $34 \%$ compared with $4 \%$ (Pelinck et al. 2002). 
Currently in IVF cycles, FSH is administered to stimulate the development of numerous preovulatory follicles to enhance the numbers of mature oocytes for recovery and thereby increase the likelihood of obtaining embryos suitable for intrauterine embryo transfer or cryopreservation (Huirne et al. 2004; Phillips et al. 2007). The application of high doses of exogenous gonadotrophins can result in prematurely raised oestrogen levels that in turn, can lead to a premature onset of the preovulatory LH surge. Therefore, a GnRH analogue is coadministered to reversibly inhibit the endogenous secretion of gonadotrophins (Smulders et al. 2010). Thereafter, exogenous LH or hCG is used to initiate the timing of ovulation. This latter approach has become the standard protocol for the induction of the final stages of oocyte maturation before oocyte retrieval (Shoham et al. 1995; Huirne et al. 2004). Without the use of GnRH analogues, LH surges occur in $~ 20 \%$ of stimulated IVF cycles which usually leads to cancellation of treatment (Edwards et al. 1996; Janssens et al. 2000).

There are two different kinds of GnRH analogues: agonists and antagonists. The difference lies in their mechanism of action. The GnRH agonists bind to the GnRH-receptors in the pituitary gland and initially stimulate an excessive release of gonadotrophins ("flare-up"). Subsequently, this leads to a decrease in the number of responsive GnRH receptors, which results in a reduced secretion of gonadotrophins. In the traditional protocol, a GnRH agonist is administered before commencing exogenous FSH and hCG treatments, thereby ensuring that the "flare-up" will be over before the gonadotrophin treatments. Conversely, a gonadotrophin antagonist may be used after exogenous gonadotrophin therapy because the antagonist binds in a competitive manner to block the receptor, thereby causing suppression of endogenous FSH and LH secretion (Tarlatzis et al. 1984). Not only do the GnRH analogues effectively prevent a premature LH-surge (Smulders et al. 2010), their combination with exogenous gonadotrophins improves the stimulation of large antral follicles, decreases treatment cancellation rates and overall, improves the efficacy of IVF treatment (Meldrum et al. 1989; Muasher 1992).

There are three main protocols involving GnRH agonist administration, namely the short, ultra-short and long protocols. In the short and ultra-short protocols, GnRH is administrated on Day one or two of the cycle for either three successive days (ultra-short protocol) or until $\mathrm{LH} / \mathrm{hCG}$ treatment (short protocol). In contrast, the long protocol involves the administration of a GnRH agonist for at least 14 to 18 days to achieve suppression of ovarian activity before commencing the administration of exogenous gonadotrophins. In the long protocol, the 
administration of the hormone agonist is continued until $\mathrm{LH} / \mathrm{hCG}$ treatment (Maheshwari et al. 2011). The long-term GnRH agonist protocol is the most commonly adopted protocol for assisted reproductive treatments worldwide (Huirne et al. 2007) due to better oocyte yield and pregnancy rate (Daya 2000; Huirne et al. 2007; Maheshwari et al. 2011).

In contrast, GnRH antagonists inhibit gonadotrophin release rapidly thereby allowing for their use at any time during the follicular phase. The large majority of protocols start the GnRH antagonist at Day six of a treatment cycle. The antagonist is commonly given by daily subcutaneous administration up to, and including, the day of hCG administration in the 'fixed time' protocol or depending on the size of the dominant follicle (in most cases, $\geq 14 \mathrm{~mm}$ ) in the 'flexible protocol' (Huirne et al. 2007; Al-Inany et al. 2011).

Often ovarian stimulation treatments include pre-treatment with oral contraceptives (OCP; consisting of oestrogen and progestogen) to facilitate the timing of egg collections, (Gonen et al. 1990), and to reduce the occurrence of large follicles before Day eight but increase the number of large follicles at the time of LH/HCG treatment. As a consequence, more oocytes are retrieved as compared with untreated women (Gonen et al. 1990; Huirne et al. 2006). The evidence also suggests that OCP pre-treatment decreases ovarian cyst formation and enables the duration of GnRH analogue treatment to be shortened without negatively affecting pregnancy rates (Biljan et al. 1998) or number of live births (Smulders et al. 2010).

In a natural menstrual cycle, the maturation of the endometrium is synchronized to the stage of follicular development and this allows for the appropriate timing of implantation of the embryo. However, hormonal treatments that lead to the growth of multiple large follicles commonly result in a suboptimal uterine environment. This has been attributed to insufficient progesterone levels during the luteal phase (Smitz et al. 1988; Balasch et al. 1991). The most common methods used to correct the deficient luteal phase are the administration of 1) progesterone (vaginally, orally or intramuscularly) alone, or in combination with oestrogen (orally) or, 2) hCG (intramuscularly or subcutaneously), in order to stimulate luteal progesterone secretion (Devroey et al. 2004; van der Linden et al. 2011). A 2008 survey revealed that vaginal progesterone is the most commonly-used method in IVF clinics around the world (Aboulghar et al. 2008) and is also the standard procedure in the protocols used by Fertility Associates (NZ). 


\subsection{The oocyte's central role in follicular development}

From recent studies, it is now evident that the oocyte does not play a passive role in the ovarian follicle, but is centrally involved in the regulation of folliculogenesis and thereby in its own development (Eppig 2001; Eppig et al. 2002). Mammalian oocytes grow and develop in an intimate and mutually-dependent relationship with adjacent follicular somatic cells (CC and mural GC). Thus, the differentiation of the $\mathrm{CC}$ is controlled by the oocyte itself and that this, in turn, affects oocyte development (Gilchrist et al. 2008). CC possess highly specialized trans-zonal cytoplasmic projections that penetrate through the zona pellucida to form gap junctions with the oocyte and results in a cumulus cell-oocyte complex (COC) (Albertini et al. 2001; Gilchrist et al. 2008). Oocyte-somatic cell communication is a bidirectional process involving gap-junctional and paracrine signalling (Eppig 2001). Specific oocyte-secreted factors (OSF) form the basis of this communication axis (Salustri et al. 1989; Buccione et al. 1990b; Eppig et al. 1993; Vanderhyden and Tonary 1995; Gilchrist et al. 2008). The most important OSF identified so far are growth-differentiation factor 9 (GDF9) and bone morphogenetic protein 15 (BMP15) which are members of the transforming growth factor $\beta$ (TGF $\beta$ ) super-family. There is very little information for OSF outside the TGF $\beta$ superfamily: one study however has reported that a critical interaction exists between BMP15 and the oocyte-secreted fibroblast growth $(F G F)$ factor 8B (Sugiura et al. 2007). These oocyte secreted factors function during at least two stages in the development of GC in mice: first, the differentiation of GC in preantral follicles to CC in antral follicles; and second, during the process of cumulus expansion initiated by the LH surge. In turn, appropriate development of $\mathrm{CC}$ promotes the development and function of the oocyte. If production of normal levels of BMP15 and GDF9 is curtailed, development and function of both the CC and the oocyte are impaired (Su et al. 2004).

\section{The role of oocyte secreted factors $(\mathrm{OSF})$}

The evidence suggests that oocytes control the rate of follicular development. Eppig et al. (2002) tested this hypothesis by separating mid-sized oocytes from secondary follicles and transferring them to primordial follicles. As a result of the oocyte transfer, the rate of follicular development was doubled and the differentiation of somatic cells was observed. Moreover, the oocytes recovered from these follicles were competent to undergo fertilization 
and embryonic development. This demonstrated that the rate of follicular development is based on the developmental program intrinsic to the oocyte (Eppig et al. 2002).

Studies in mice show that oocytes also influence follicular development by regulating kit ligand (KL) secretion. KL is produced by pre-antral GC and promotes oocyte growth through the Kit receptor located on the oocyte (Packer et al. 1994). In vivo, oocyte-regulated expression of $K L$ mRNA is related to the stage of oocyte development. Growing oocytes stimulate, whereas fully grown oocytes suppress, GC expression of $K L$ mRNA (Joyce et al. 1999). Further evidence suggests that the level of $K L$ mRNA expression is controlled by both paracrine and hormonal factors. Moreover, the overall expression and the relative abundance of KL isoforms are influenced by GDF9, BMP15 and FSH at various stages of follicular development (Thomas and Vanderhyden 2006).

The oocyte actively directs the extent of differentiation of CC and mural GC phenotypes most likely via a concentration gradient of OSF (Hussein et al. 2005). During follicular growth the mural GC are inhibited from luteinisation and progesterone synthesis by the OSF however they maintain their steroidogenic activity of synthesizing oestradiol from precursor steroids under the influence of FSH (el-Fouly et al. 1970; Vanderhyden et al. 1993; Vanderhyden and Tonary 1995; Eppig et al. 1997b). In the absence of OSF, the default pathway of GC differentiation is toward a luteal (progesterone-secreting) phenotype (Eppig et al. 1997a; Li et al. 2000; Gilchrist et al. 2008). Elimination of oocyte paracrine signalling either by physical removal of the oocyte or by blocking the signalling pathway through which GDF9 and BMP15 act (i.e. SMADs) in CC, causes CC to lose their distinctive phenotype and to function more like mural GC. However, CC characteristics can be fully restored by treating these cells with OSF, demonstrating also, that the oocyte actively abrogates FSH-induced luteinisation of CC (Gilchrist et al. 2008). Further evidence suggests that in mouse follicles, OSF interact with oestradiol to promote the development and function of CC (Sugiura et al. 2010). COC cultured in vitro rapidly lose their ability to maintain oocyte meiotic arrest unless cultured in oestradiol-containing media, indicating the importance that oestradiol may have in maintaining a CC phenotype and oocyte meiotic arrest in Graafian follicles in mouse ovaries in vivo (Zhang et al. 2011).

During the final stages of follicular development just before ovulation, the COC undergoes mucification and expansion (Figure 1E). This process is initiated by gonadotrophins or 
epidermal growth factor (EGF)-like peptides and is regulated by OSF that enables the CC to synthesize extracellular matrix (ECM) molecules in response to the gonadotrophin/EGF signal (Dekel and Kraicer 1978; Eppig 1979; Salustri et al. 1989; Buccione et al. 1990b; Eppig et al. 1993). CC expansion is essential for ovulation and successful fertilization. The failure to synthesize components of the cumulus matrix results in suboptimal cumulus expansion leading to reduced fertility or sterility (Russell and Robker 2007; Kolle et al. 2010).

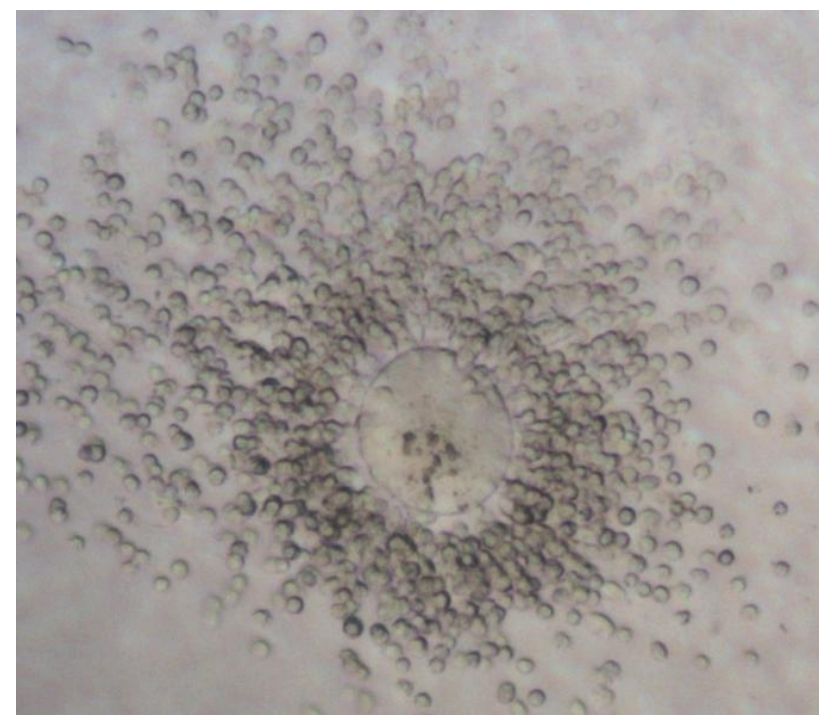

Figure 1E: High resolution image (200 x magnification) showing an expanded mouse COC. The oocyte in the middle is surrounded by multiple layers of CC. Image supplied by Dr Janet Pitman.

OSF also actively prevents CC death. In antral follicles undergoing atresia, the COC is the last compartment of the ovarian follicle to undergo apoptosis (Yang and Rajamahendran 2000), most likely by establishing a morphogenic gradient of OSF, particularly BMP15 and BMP6 (Hussein et al. 2005). Under in vitro experimental conditions, the prevalence of apoptosis in COC is the lowest in immediate proximity of the oocyte and highest in the most outer layers. FSH also prevents apoptosis in CC and GC, and its actions are additive to those of OSF. It was revealed that oocytes prevent apoptosis within $\mathrm{CC}$ by altering the ratio of BAX to BCL-2 in favour of cell survival (Hussein et al. 2005).

Oocytes regulate metabolic cooperativity with CC and GC (Sugiura et al, 2005). In the mouse at least, glycolysis in oocytes is lacking and is dependent on the provisions of glycolysis 
products such as pyruvate from the $\mathrm{CC}$ as an energy source for growth and resumption of meiosis (Biggers et al. 1967; Donahue and Stern 1968). In turn, oocytes regulate the extent of glycolysis and the tricarboxylic acid (TCA) cycle in GC/CC relative to the stage of growth and development of the oocyte. Therefore, this indicates a metabolic cooperativity between oocytes and CC/GC whereby oocytes require energy substrates from the CC/GC and in turn, the OSF regulate somatic cell function and in turn, follicular development (Sugiura et al. 2005) in a developmentally-coordinated manner.

Paracrine factors from fully grown oocytes also stimulate the acquisition of amino acids from CC (Eppig et al. 2005). Certain amino acids (e.g. L-alanine) required for protein synthesis are supplied to the oocyte from the CC via gap junctions (Buccione et al. 1990a). In the mouse, the expression of amino acid transporters, such as solute carrier family 38 (Slc38a3) mRNA in $\mathrm{CC}$ is regulated by the oocytes according to the stage of oocyte development. Slc38a3 is a sodium-coupled neutral amino acid transporter and transporters from this family exhibit a preference for L-glutamate, L-histidine and L-alanine.

Recent studies of genetic mutations in sheep also highlight the importance of OSF in regulating ovarian follicular development and ovulation rate. Alterations to the concentrations of GDF9 and BMP15 protein in vivo leads to significant changes in ovulation rate in sheep (Galloway et al. 2000; McNatty et al. 2003). These findings, together with immunization results with BMP15 and GDF9 peptides, demonstrate that by altering the bioavailability of GDF9 and BMP15 in vivo, it is possible by exogenous means to enhance ovulation rate in sheep, cattle and mice (Juengel et al. 2002; Juengel et al. 2009; McIntosh et al. 2012) and increase lamb production or alternatively induce infertility in sheep (McNatty et al. 2004).

The importance of OSF on oocyte quality was tested under in vitro maturation (IVM) conditions. When bovine COC were co-cultured with denuded oocytes or were cultured in media pre-conditioned with OSF, a $\sim 20 \%$ increase ( $\sim 60 \%$ compared to $\sim 40 \%$ ) in blastocyst formation rate was observed. Moreover, the addition of OSF to the culture media also improved subsequent embryo quality as evidenced by total cell and trophectoderm cell numbers (Hussein et al. 2006). Similarly in mice, oocytes from COC treated with OSF (GDF9) during IVM, resulted in blastocysts with significantly increased total cell and inner cell mass (ICM) cell numbers. Although blastocyst formation rates were similar, the 
percentage of hatching blastocyst per cleaved embryo was significantly increased in culture media supplemented with exogenous GDF9. Whilst the addition of GDF9 to COC cultured in vitro had no effect on implantation rate, the embryos derived from the GDF9-treated group had almost doubled the rate of foetal survival compared to controls (Yeo et al. 2008). Therefore, under in vivo conditions, the reduction of biologically active GDF9 or BMP15 both increases ovulation rate, oocyte yield and lambs born whereas under in vitro conditions, supplementary OSF increased the numbers of blastocysts recovered following IVM of oocytes. This represents a paradox that remains to be resolved.

\subsection{The outcome of human oocytes following oocyte collection: IVF, pre- implantation genetic diagnosis and fertility preservation}

The main reasons why women undergo ovarian-stimulation and oocyte collection are: 1) they are unable to conceive naturally; 2) are at a high risk of transmitting genetic defects to their offspring and; 3) to preserve their fertility. In the first two scenarios, the most common treatment is to recover COC via a transvaginal retrieval method followed by IVF on the same day, whilst in the third case, oocytes are cryopreserved after COC retrieval and IVF usually takes place months or years later.

\section{IVF treatment and associated therapies for increasing healthy offspring outcomes}

Based on the most commonly used definition of infertility (lack of pregnancy after one year of unprotected regular intercourse), 10-15\% of reproductive age couples are diagnosed with infertility (Forti and Krausz 1998). For these couples, IVF offers the best chance of having a healthy offspring (Reindollar et al. 2010). Over the last decade the annual increase in ART services has been estimated to be $5-10 \%$ in developed countries (Chambers et al. 2009). The increasing demand for assisted reproduction in Western countries can be attributed to various factors such as the tendency of delaying childbearing, an increase in the effectiveness of ART, an increased awareness for these treatments and in some cases demographic strategies aimed at reducing declining fertility rates (Forti and Krausz 1998; Ziebe and Devroey 2008). Legislation, public funding and treatment costs vary significantly amongst countries (Collins 2002; Chambers et al. 2009). The utilization of ART services depends highly on availability, cost and consumer price, as IVF and intra-cytoplasmic sperm injection (ICSI) treatments are expensive technologies that employ highly skilled professionals from multiple fields of 
practice and involve sophisticated laboratory facilities (Collins 2002; Chambers et al. 2009). It was reported that even in developed countries, treatment utilization rates are strongly correlated to low treatment costs or generous public funding (Chambers et al. 2009).

The average chance of conception for normally fertile couple having regular, unprotected intercourse is approximately $25 \%$ during each menstrual cycle. In most couples, conception occurs within a year of unprotected intercourse. If pregnancy has not been achieved within this period, an underlying pathological condition is sometimes at cause (De Sutter 2006). The causes of infertility can be divided if four major categories: 1) the female factor (i.e. ovulatory disorders, tubal disease and endometriosis) affecting approximately $35 \%$ of cases ; 2) male factor (i.e. low sperm concentrations, poor sperm motility and/or morphology) present in $30 \%$ of couples; 3) combined factors present in $20 \%$ of couples; and; 4) unexplained infertility in about $15 \%$ of the cases (Forti and Krausz 1998). In these instances, couples have little hope of having children by natural conception and they form the base of people seeking help in the form of assisted reproduction.

Another reason why couples seek IVF treatment is to assess the genetic condition of their embryos and to select only unaffected embryos for replacement (Basille et al. 2009). The majority of couples undergoing preimplantation genetic diagnosis (PGD) treatment are usually those with a high probability of conceiving a child with an incurable disease. Most of the time, these couples have a traumatic obstetrical history: birth of a child with genetic disease or previous termination of pregnancy after chorionic villus sampling or amniocentesis. Secondly, they are couples with genetic disease, either known from family history or discovered during infertility investigations (Basille et al. 2009).

\section{Fertility preservation}

The most common reasons why women seek fertility preservation are due to the need for cancer therapy and for social reasons (Paynter 2000; Goold and Savulescu 2009). Currently, oocyte cryopreservation is the best option available for women to preserve their fertility. This method facilitates the long-term storage of gametes and allows greater flexibility in fertility services (Paynter 2000). Cryopreservation below $-130{ }^{\circ} \mathrm{C}$ allows virtually indefinite storage of cells and tissues (liquid nitrogen at a temperature of $-196^{\circ} \mathrm{C}$ is the usual holding medium) (Paynter 2000). By 2009, cryopreservation allowed for around 4,300 oocytes to be collected 
from which more than 80 children thus far have been born (Maltaris et al. 2009). Several recent studies confirmed that oocytes frozen by current cryopreservation methodologies have similar success rates to those from fresh IVF cycles or frozen embryos replacements (Noyes et al. 2010). Moreover, it was reported that the mean birth weight and the incidence of congenital anomalies from children born from vitrified oocytes are similar to those of spontaneous conceptions in fertile women or infertile women undergoing IVF treatment (Chian et al. 2008).

\section{$1.7 \quad$ Oocyte quality}

Development of the human embryo begins with the fusion of spermatozoon and oocyte, the epigenetic reprogramming of the pronuclei of the gamete and a series of cleavage divisions that culminate with the activation of the embryonic genome (Wong et al. 2010). The oocyte occupies a central role in the development of the embryo. This role is acquired progressively during oogenesis, through a range of cellular and molecular attributes that provide the oocyte with the ability to complete meiosis, ensure normal fertilization, undergo preimplantation development and accomplish successful implantation as a consequence of the action of stored maternal mRNAs and proteins (Coticchio et al. 2004). The finding that blastocyst development can be predicted before embryonic genome activation suggests the critical role that oocytes and maternal transcripts have in the successful outcome of an embryo (Wong et al. 2010). Utilization of the most competent oocytes during IVF is crucial to ensure the derivation of high-quality embryos and successful pregnancy. This is especially the case for human IVF given the very low $(<7 \%)$ oocyte to live baby rate ( $\mathrm{Li}$ et al. 2008; Patrizio and Sakkas 2009).

\section{Factors associated with oocyte quality}

\section{Antral follicle count}

The antral follicle count refers to the number of antral follicles in the ovary that measure 2-5 $\mathrm{mm}$ in diameter at the beginning of the menstrual cycle (day one-two) as determined from an ultrasound scan. There is a highly significant correlation between the antral follicle count and the ovarian response to exogenous gonadotrophin stimulation with respect to the number of oocytes retrieved and the utilization rate of embryos in the same cycle (Chang et al. 1998). Conversely, a decreasing antral follicle count correlates with decreasing pregnancy rate. 
Patients who have a low antral follicle count require more gonadotrophins for ovarian stimulation, which increases costs. Moreover, IVF treatments in these women result in higher treatment cancellation rates and lower pregnancy outcomes (Chang et al. 1998; De Sutter and Dhont 2003; Saldeen et al. 2007).

\section{$B M I$}

Offering patients elective, publicly funded procedures in New Zealand is done according to clinical priority access criteria (CPAC) which was introduced in the mid-1990s for several procedures (Hadorn and Holmes 1997). In 2000, a new section covering assisted reproduction was added to the CPAC with the objective of providing a mechanism for rationing limited public funds. The seven criteria developed for CPAC included some of the most common factors known to influence oocyte quality and pregnancy outcome, such as women's age $(<40$ years), BMI ( $\left.\leq 32 \mathrm{~kg} / \mathrm{m}^{2}\right)$ and basal serum FSH level ( $<15 \mathrm{IU} / \mathrm{mL}$ ) (Elective Services 2011). A 2008/2009 survey by the New Zealand Ministry of Health based on the 2007 World Health Organization (WHO) criteria concluded that in females of 15 years and older, $1.5 \%$ were underweight, $37.9 \%$ were normal weight, $32.8 \%$ were overweight and $27.8 \%$ were obese. Moreover, the prevalence of obesity in 19-30 year old and 31-50 year old women is $25.1 \%$ and $27.9 \%$, respectively (University of Otago and Ministry of Health 2011). Studies that investigated the effect on increased BMI on delivery rate revealed that women with a BMI of $>32 \mathrm{~kg} / \mathrm{m}^{2}$ had a $38 \%$ birth rate, whilst those with a BMI of $<32 \mathrm{~kg} / \mathrm{m}^{2}$ had a $52 \%$ birth rate following a treatment-related pregnancy or spontaneous pregnancy (Gillett et al. 2006). Further, clinical trials revealed that treatment cancellation, poor response and gonadotrophin use per cycle rose with increasing BMI. More importantly, the pregnancy rate amongst obese women was lower compared with normal weight women following IVF treatment (Orvieto et al. 2009; Pinborg et al. 2011).

Age

Previous studies showed that with increasing patient age, there is a highly correlated decrease in antral follicle count (Chang et al. 1998). Moreover, genetic studies confirmed that genomic abnormality rates in oocytes increase with age: embryos from women 37 years and older have a higher incidence of chromosomal abnormalities (Gianaroli et al. 1999; Wells and Delhanty 2000). Human IVF studies have confirmed that the pregnancy rate following IVF is significantly lower in women $\geq 40$ years of age, decreases sharply in women $>42$ and is essentially absent in women $>45$ years of age (Lass et al. 1998). Other studies investigating 
the links between oocyte development and live births reveal that while women $\leq 37$ years of age have an oocyte to baby rate of 4.3-4.5\% following IVF, their 41-42 year old counterparts have significantly less at $1 \%$ (Patrizio and Sakkas 2009). The low reproductive potential of aging oocytes was further confirmed by the high embryo wastage rate, where $95 \%$ of the embryos transferred in women over 40 years of age failed to result in a live birth (Bromer et al. 2009).

\section{Basal serum FSH levels}

The rise of basal serum FSH levels begins on average a decade or more before menopause begins. A significant progressive increase in FSH levels as early as age 29-30 years can be detected in women with regular ovulatory cycles. This increase continues throughout the $30 \mathrm{~s}$ and becomes more marked in the early 40s (Ahmed Ebbiary et al. 1994). Increased basal serum FSH levels are regarded as a basic endocrine marker of reproductive ageing. There is a high correlation of antral follicle count and serum FSH levels at Day 3 of the menstrual cycle due to antral follicles being the main source of inhibin-B secretion and its subsequent negative feedback effects on the pituitary gland. As a consequence, a decrease in the number of antral follicles leads to increases in the serum FSH levels (Cahill et al. 1994; Groome et al. 1996).

\section{Serum Anti-Müllerian hormone (AMH) levels}

Anti-Müllerian hormone (AMH) is a member of the TGF-beta super-family. It is synthesized by GC in women, and by Sertoli cells in the testes of men (Vigier et al. 1984; Josso 1990). During foetal development, its principal function is to induce regression of the Müllerian ducts during male sex differentiation; however in women it appears to play an important role in folliculogenesis (Durlinger et al. 2002). During follicular development, expression of AMH is low in primary follicles and high in secondary, and small antral follicles $(\leq 4 \mathrm{~mm}$ in diameter) but levels gradually decline again in larger antral follicles (4-8 mm) (Weenen et al. 2004). During in vitro culture of human ovarian tissue, the presence of AMH in the media is significantly correlated with the proportion of follicles that have initiated growth (i.e. primary and secondary follicles). This finding suggests that $\mathrm{AMH}$ is a functional marker for early growing follicles (Schmidt et al. 2005). Moreover, since AMH is produced only by growing ovarian follicles, and the number of growing follicles is proportional to the size of the primordial pool, it was proposed that $\mathrm{AMH}$ could be used as a measure of the size of the ovarian reserve (van Rooij et al. 2002). Indeed, following IVF treatment it was observed that 
there is a highly significant correlation between serum AMH levels and ovarian reserve. Low baseline serum AMH concentrations were associated with a low number of oocytes retrieved following IVF treatment that was consistent with, and indicative of, a diminished ovarian reserve. Indeed the AMH test was found to have a predictive performance comparable with that of an antral follicle count. The testing of serum AMH levels during human IVF procedures as a predictor of ongoing pregnancy however appears to be somewhat limited since AMH levels do not assess oocyte quality (van Rooij et al. 2002).

\section{PCOS}

Polycystic ovarian syndrome is the most common endocrine disorder, affecting approximately 5-7\% of women of reproductive age (Knochenhauer et al. 1998; Asuncion et al. 2000). In 2003, the Rotterdam consensus established the most recent diagnostic criteria. According to this consensus, women are diagnosed with PCOS, if they are affected by at least two of the following three features: 1) oligo/anovulation; 2) hyperandrogenism characterized clinically by hirsutism, acne, and androgen-dependent alopecia and elevated serum concentrations of androgens, particularly testosterone and androstenedione (Goldzieher and Green 1962) and; 3) polycystic ovaries (Rotterdam ESHRE/ASRM consensus 2004). PCOS is typically associated with hyper-secretion of LH and androgens but normal or low serum concentrations of FSH (Yen et al. 1970; Holte et al. 1994). Moreover, insulin resistance and hyperinsulinaemia are conditions frequently associated with PCOS (Legro et al. 1999). Previous studies suggested that women with PCOS may be subfertile and oocyte developmental potential could be compromised. Indeed, insulin-mediated glucose metabolism is abnormal and lactate production is impaired in granulosa-lutein cells following IVF of oocytes from women with PCOS (Barbieri et al. 1988). Moreover the enhanced response to LH-stimulated progesterone production adds further evidence to the hypothesis that raised insulin, LH and androgen concentrations in women with PCOS are all involved in premature differentiation and luteinisation of GC and possibly the arrest of follicle growth (Willis et al. 1998; Rice et al. 2005). Indeed, morphologically indistinguishable MII oocytes from PCOS and healthy women have been reported to exhibit different gene expression profiles, including genes involved in the meiotic or mitotic cell cycle and maternally linked genes (i.e. NLR family, pyrin domain containing 5 (NALRP5) gene, required for early embryogenesis) (Wood et al. 2007). 
The majority of clinical studies investigating pregnancy rates in women with PCOS report similar results compared to healthy women following multiple-embryo replacements (Esinler et al. 2005; Sahu et al. 2008; Swanton et al. 2010; Mellembakken et al. 2011). However in IVF treatments where more than 20 oocytes were collected, the clinical and ongoing pregnancy rates were significantly reduced in women with PCOS (Weghofer et al. 2007). In addition, the clinical abortion rate/ pregnancy loss in these women were significantly higher compared with reproductively-normal women, indicating that oocyte or/and uterine quality may be impaired (Sagle et al. 1988; Carmina and Lobo 1999; Ludwig et al. 1999).

\subsection{Selection of healthy oocytes}

Non-invasive selection of developmentally competent human oocytes would represent a very significant technological advance. A reliable selection method could enhance the efficiency of human assisted reproduction and would be particularly relevant in countries where legal, social or religious factors restrict the number of oocytes that can be inseminated or embryos selected (Rienzi et al. 2011). Furthermore, the use of reliable oocyte quality markers would allow better planning of assisted reproductive treatments for women where cryopreservation of embryos is not a possible option (i.e. women undergoing cancer therapy or single women) and the collection and cryopreservation of mature oocytes gives the best chance for establishing a family in the future (Paynter 2000; Goold and Savulescu 2009). The selection and the cryopreservation of eggs would also alleviate many of the ethical concerns associated with embryo preservation (Goold and Savulescu 2009).

There is very limited information about the financial cost of an IVF cycle. Most of the data published on financial implications of IVF are patient related (Bouwmans et al. 2008), however it is equally important for cost-effectiveness from the perspective of the provider. A reliable selection for good quality oocytes for insemination has the potential of maximizing time and financial spending of clinics for one cycle. It is not unreasonable to think, that in the future with the selection of oocytes, follicle aspirations, inseminations and oocyte transfers could be programmed for the same day, thus organizations no longer requiring facilities and culture related expenses (i.e. media) for embryo culture. Furthermore, it would allow for the maximizing of patient numbers in accessing treatments and minimizing staff involved in care and administration of treatment. A further benefit of early oocyte or embryo selection would be to minimize the potential harmful effects (epigenetic reprogramming) of extended in vitro 
culture on the long-term health and physiology of the child born (Le Bouc et al. 2010; Calle et al. 2012).

\section{Oocyte selection according to morphological characteristics}

Morphological assessment of embryos at the preimplantation stage is a key element of the laboratory work in human assisted reproduction. Routine evaluations that follow internationally-acknowledged criteria are performed at predetermined checkpoints by microscopy. The current view of most human embryologists is that all MII oocytes that meet the basic criteria (about $85 \%$ of oocytes collected) should be subjected to fertilization procedures. The morphological evaluation of oocytes by light microscopy is rather more inexact compared with that for embryos. Nevertheless, even embryo selection based on morphological and developmental characteristics is a superficial method, as seemingly morphologically 'normal', but intrinsically handicapped, embryos are routinely cultured and transferred resulting in compromised in vitro development, low pregnancy rates, abortions and further negative consequences (Rienzi et al. 2011).

The application of exogenous ovarian treatments in human reproduction further complicates the situation. In contrast to the in vivo process, where oocyte maturation occurs as the result of a long and meticulous natural selection procedure, common ovarian stimulation procedures override this process allowing for the seemingly successful maturation of oocytes but with inherently compromised quality. These are often destined for fertilization failure, compromised embryo development or long-term consequences in vivo (Rienzi et al. 2011).

Amongst human embryologists, it is widely accepted that routine morphological investigations are simply unable to predict developmental competence and fertilization potential of the oocyte. Correlations between the morphological characteristics of the COC, structure of the zona pellucida, size of perivitelline space, morphology of the first polar body, shape of the oocyte and appearance of the ooplasm with that of oocyte developmental competence revealed inconclusive and often contradicting results (Rienzi et al. 2011). The most promising morphological assessment appeared to be visualization of the meiotic spindle by polarized light microscopy (Petersen et al. 2009). However, this technique offers limited selection potential as meiotic spindles can be imaged in about $90 \%$ of MII oocytes collected (Rienzi et al. 2003) increasing the efficiency of selection by only $10 \%$. Also, this technique 
requires strict implementation of protocols due to parameters such as time of analysis, oocyte rotation, temperature and other environmental conditions (e.g. $\mathrm{pH}$ ) compromising spindle visualization (Petersen et al. 2009).

\section{Oocyte selection according to COC morphology}

It was proposed that morphological grading of the degree of expansion of the $\mathrm{CC}$ mass could be related to developmental potential of associated oocytes. However, studies that investigated the degree of expansion in relation to oocyte maturity, fertilization, embryo cleavage and pregnancy outcome concluded that there was no or little association between the degree of expansion of the CC mass and maturity of the oocyte. Although, it appeared that women who had a higher proportion of expanded COC had a better pregnancy outcome, COC grading performed poorly and was deemed unsuitable as a marker for the selection of single good quality oocyte with high chance in pregnancy (Ng et al. 1999; Rattanachaiyanont et al. 1999).

Similarly, authors who investigated the incidence of apoptosis in $\mathrm{CC}$ in relation to oocyte quality, showed that the proportion of apoptosis in $\mathrm{CC}$ is relatively similar across samples, and is not correlated to blastocyst development and/or pregnancy/live birth outcome (Lee et al. 2001; Host et al. 2002; Moffatt et al. 2002; Corn et al. 2005). It can be concluded that morphological evaluation of CC apoptosis provides limited prognosis on oocyte quality and is an unreliable marker in human IVF.

\section{Oocyte selection according to cumulus cell gene expression}

The need to develop objective and reliable non-invasive predictors of oocyte developmental competence has led researchers to investigate $\mathrm{CC}$ as potential biomarkers of oocyte quality. As discussed previously, the developmental competence of an oocyte is closely associated with follicular somatic cell development, in which the oocyte plays an active role in regulating the surrounding $\mathrm{CC}$. In return, $\mathrm{CC}$ provide essential support for the development and maturation of oocytes. The identification of key molecules and signalling pathways within the COC regulatory loop could provide new insights into the mechanism underlying oocyte development and novel molecular predicators for the selection of high quality oocytes (Li et al. 2008). 
In the late 1980s and early 1990s, new polymerase chain reaction (PCR) technologies emerged, that allowed the relative and absolute quantification of nucleic acids (Wang et al. 1989; Noonan et al. 1990). Early publications demonstrated that the quantitative capability of PCR was similar to classical methods such as Northern blot or slot blot analyses (Murphy et al. 1990; Noonan et al. 1990). Moreover, quantitative PCR (QPCR) has been shown to correlate with in-situ hybridization results, where equivalent proportionality in the relative amounts of RNA was obtained by both methods (Park and Mayo 1991). Indeed, if optimization and validation of the method is done properly, QPCR is a very powerful quantitative tool (Ferre 1992).

In 2004, McKenzie et al. proposed for the first time, that gene expression levels in human CC measured by QPCR could potentially act as quality markers for the associated oocytes. For the first time, individual human $\mathrm{CC}$ samples were subjected to mRNA extraction using sensitive commercial RNA isolation kits with the intention to quantify selected target genes using QPCR methods (McKenzie et al. 2004). Further investigations by QPCR and microarray technologies have revealed that gene expression in $\mathrm{CC}$, and specifically transcripts related to the down-stream effects of OSF, in particular GDF9 and BMP15 mRNA, are correlated with oocyte development (Hasegawa et al. 2005; Zhang et al. 2005; Cillo et al. 2007; Feuerstein et al. 2007; Assou et al. 2008; Hamel et al. 2008; van Montfoort et al. 2008; Anderson et al. 2009; Adriaenssens et al. 2010; Assidi et al. 2011; Gebhardt et al. 2011; Wathlet et al. 2011).

The first genes that have been investigated were not surprisingly, genes responsible for the synthesis of the COC extracellular matrix proteins, hyaluronan synthase 2 (HAS2), pentraxin 3 (PTX3), tumour necrosis factor $\alpha$-induced protein 6 (TNFAIPO) and genes involved in cumulus cell signalling such as gremlin 1 (GREM1) and prostaglandin-endoperoxide synthase 2 (PTGS2) (McKenzie et al. 2004). Other CC genes in subsequent studies showed some correlation to positive outcomes are those involved in steroidogenesis (3-beta-hydroxysteroid dehydrogenase $1(3 \beta H S D 1)$ and ferrodoxin 1 ( $F D X 1)$ involved in progesterone synthesis, cytochrome $\mathrm{P} 450$ aromatase (CYP19A1) responsible of metabolizing androgen into oestradiol-17ß) (Hamel et al. 2008), in neuronal traffic through the cytoskeleton (neuropilin 1 $(N R P 1))$ and cell cycle and signalling (ubiquilin 1 (UBQLN1) and cyclin D2 (CCND2)) (van Montfoort et al. 2008; Assidi et al. 2011). 
Currently, many more genes have been investigated, such as those involved in the secretion of the extracellular matrix (versican) of the COC, cumulus cell signalling (for example, amphiregulin, connexin 43, aryl hydrocarbon receptor, steroid sulfatase, steroidogenic acute regulatory protein, syndecan 4 and calmodulin 2) and metabolic pathways (for example, stearoyl-CoA desaturase 1 and 5, aldolase A, lactate dehydrogenase A, phosphofructokinase and pyruvate kinase) (Feuerstein et al. 2007; Adriaenssens et al. 2010; Gebhardt et al. 2011; Wathlet et al. 2011).

Although the above mentioned studies similarly suggested that possible links between CC gene expression and embryological outcome exist, the results are variable and more in-depth studies were required (Zhang et al. 2005; Cillo et al. 2007; Feuerstein et al. 2007; Assou et al. 2008; Anderson et al. 2009). A possible reason for these variable results could be due to biological and treatment variables. The first study to question the relationship between biological variables and CC gene expression ( $P R$ gene) was in 2005, when the effect of follicular characteristics such as size and steroid concentrations in follicular fluid were investigated (Hasegawa et al. 2005). Although no significant associations were found, later studies revealed that other biological variables such as age may alter gene expression in both oocytes (Grondahl et al. 2010) and CC (Adriaenssens et al. 2010). It was further suggested that BMI, serum FSH concentrations, the gonadotrophin preparation used for ovarian stimulation and serum oestradiol concentrations at the time of the ovulation trigger, also alter the expression levels of certain genes in CC (Adriaenssens et al. 2010). Indeed, recent studies have confirmed that increased basal serum FSH levels do indeed affect the levels of gene expression in COC and that it might be one of the possible causes behind the reduced fertility potential in young women with diminished ovarian reserve (Greenseid et al. 2011; Jindal et al. 2012).

Most importantly however, none of these studies have identified a reliable assessment system or molecular marker capable of efficiently selecting from a total pool of MII oocytes, those that will go on to form successful pregnancies. Whilst the above mentioned studies carried out their investigations using SYBR Green or TaqMan singleplex QPCR, recent investigations revealed that single oocytes and individual $\mathrm{CC}$ masses provide sufficient mRNA for quantification by multiplex QPCR (Crawford and McNatty 2012; Crawford \& McNatty; unpublished results). The multiplex QPCR method has the advantage of 
simultaneously measuring multiple genes in a single reaction, saving time and maximizing the use of minimal amounts of template available. However this method has yet to be tested in human CC studies.

\subsection{Aims of study}

The objectives of this research programme were to:

1. Test the feasibility of multiplex QPCR technology to measure the expression of numerous candidate genes in individual CC masses during collection of COC for IVF. This includes the validation of an appropriate housekeeping gene for human follicular tissues. In this context, the housekeeping properties of two widely used reference genes were investigated: ribosomal RNA $18 S$ and 60S ribosomal protein L19 (RPL19).

2. Investigate $\mathrm{COC}$ morphology and $\mathrm{CC}$ numbers in relation to embryo development and pregnancy outcome in women undergoing IVF treatment with ICSI.

3. Assess the expression levels of candidate genes in $\mathrm{CC}$ in relation to embryo development and pregnancy outcome in women undergoing IVF treatment with ICSI.

To fulfil the aims of Objective 3, eight CC-derived candidate genes were selected as potential oocyte quality markers on the basis that: several had previously shown promise in human $\mathrm{CC}$ studies (McKenzie et al. 2004; Cillo et al. 2007; Assidi et al. 2011; Gebhardt et al. 2011; Wathlet et al. 2011) or; were genes that might be influenced by the oocyte during the final steps of folliculogenesis and ovulation (Otsuka et al. 2005; Hernandez-Gonzalez et al. 2006; Robker et al. 2009). The eight candidate genes selected for this study were:

hyaluronan synthase 2 (HAS2) which is known to be critical for the formation and expansion of the CC mass and previously shown to be correlated with early embryological development (McKenzie et al. 2004; Cillo et al. 2007) and to have a positive correlation with age (Adriaenssens et al. 2010);

follicle stimulating hormone receptor $(F S H R)$ in which expression levels have been shown to be regulated by OSF which in turn are thought to mediate the actions of FSH on follicular cells (Otsuka et al. 2005); 
solute carrier 2, member 4 (SLC2A4, also known as GLUT4) which is associated with energy metabolism in COC and is previously reported to be one of the glucose transporters in CC (Roberts et al. 2004);

versican $(V C A N)$ is a major component of the COC extracellular matrix, previously reported as one of the most promising oocyte quality markers in CC (Adriaenssens et al. 2010; Gebhardt et al. 2011; Wathlet et al. 2011);

activated leukocyte cell adhesion molecule (ALCAM) mediates homophilic (ALCAM ALCAM) and heterophilic (ALCAM-CD6) cell to cell interaction, is known to be involved in the initial step of human embryo implantation (Fujiwara et al. 2003), expressed in CC and is a potential oocyte quality marker (Adriaenssens et al. 2010; Wathlet et al. 2011);

secreted frizzled-related protein 2 (SFRP2) is a soluble modulator of Wnt signalling, and has been reported to be expressed in mice CC at peak levels in ovulated COC (Hernandez-Gonzalez et al. 2006);

progesterone receptor $(P R)$ is expressed in the dominant follicle and the active corpus luteum of women and is essential for a successful implantation although little is known about the role of this gene during the peri-conception period (Robker et al. 2009) and;

neuropilin 1 (NRPl) is a membrane-bound co-receptor to tyrosine kinase receptor for vascular endothelial growth factor and members of the semaphorin family proteins. Neuropilin 1 is involved in the angiogenic and neuronal traffic through the cytoskeleton, in cell assembly and interaction and more importantly in vesicle guidance and transport in follicles following oestradiol induction. Moreover, in a preliminary study it was reported that up-regulation of $N R P 1$ expression in CC may potentially indicate an oocyte with a positive pregnancy outcome (Assidi et al. 2011). 


\section{Chapter 2 METHODOLOGY}

\subsection{Ethical approval}

In New Zealand, health and disability research involving humans requires ethical approval. The review of health and disability research is carried out by seven Health and Disability Ethics Committees. The Multi-region Ethics Committee considers applications for research that is to be carried out in more than one of the four Ethics Committee regions (http://www.ethicscommittees.health.govt.nz/).

Fertility Associates clinics operate in four different locations: Wellington, Christchurch, Auckland and Hamilton. For the studies reported herein, ethical approval was requested for Fertility Associates Wellington clinic, and granted by the Multi-region Ethics Committee on 17 November 2008 until November 2013 (Permit number MEC/08/10/121). All persons (women and partners) that were participated in this study provided informed consent (Appendix 1).

\subsection{Subjects}

The subjects of this study were women undergoing IVF treatment. Patient enrolment was undertaken by clinicians at Fertility Associates under the management of Prof. John Hutton. For this study, four different groups were established. The groups were identified according to the likely causes of infertility.

All Groups (except Group 4) were to be aged $\leq 37$ years;

All Groups (except Group 2) were to have a BMI of $<28$, and no evidence of androgenicity

\section{Group 1: Young and healthy women}

Period Cycle

Reason for Infertility

Day 2 FSH (Basal E2)

FSH stimulation
Regular 26-32 days

Male Factor or Tubal Disease

$<9 \mathrm{iu} / 1$

stimulation protocol: '5.85' (see below)

daily dose of FSH: 150IU 
IVF treatment (cycle) number: 1 or 2 (women who qualified for the above criteria, but had two or more previous IVF treatments were not enrolled).

\section{Group 2: Young women with PolyCystic Ovarian Syndrome (PCOS)}

The diagnosis of PCOS was based on the 2003 Rotterdam ESHRE/ASRM consensus (2004). Patients were enrolled in this group if they were diagnosed by the presence of at least two of the following conditions: oligo/anovulation, hyperandogenism and polycystic ovaries.

Period Cycle

Medical Disease

Reason for Infertility

Day 2 FSH (Basal E2)

FSH stimulation

IVF treatment number:
Irregular/unpredictable

None indicated

PCOS (but can also include male factor/icsi)

$<9 \mathrm{iu} / 1$

Stimulation protocol: ' 5.85 ' (see below);

Daily dose of FSH: 150IU, or preferably less

1 or 2 (women who qualified for the above criteria, but had two or more previous IVF treatments were not enrolled).

\section{Group 3: Young women with diminished ovarian reserve}

Reason for Infertility Low antral follicle count (but can also include male factor/ICSI)

Day 2 FSH (Basal E2) >9 iu/l

Antral Follicle Count $\quad<$ or $=4$ each ovary

FSH stimulation Stimulation protocol: '5.9' or '5.85' (see below)

Daily dose of FSH: $225 \mathrm{IU}$ or $300 \mathrm{IU}$

IVF treatment number: $\quad 1$ or 2 (women who qualified for the above criteria, but had two or more previous IVF treatments were not enrolled).

\section{Group 4: Older and healthy women}

Age

Period Cycle

Reason for Infertility

Day 2 FSH (Basal E2)

Antral Follicle Count

FSH stimulation $\geq 40$ years

Recorded - not restricted

Not endometriosis or PCOS

Recorded - not restricted

Recorded - not restricted but not PCOS

Stimulation protocol: '5.9' or '5.85' (see below) 
Daily dose of FSH: 225IU or 300IU

IVF treatment number: $\quad$ Not restricted

The following background information was recorded from women undergoing treatment:

Blood and scan results: FSH (IU/L), AMH (ng/mL), LH (basal) (IU/L), testosterone (for PCOS patients), oestradiol (pmol/L) levels during stimulation.

Physical condition: height, weight, BMI $\left(\mathrm{kg} / \mathrm{m}^{2}\right)$.

Other drug treatments: diabetes, asthma, blood pressure, other.

Lifestyle factors: smoker/ non-smoker.

Ethnicity: Maori, Pacifica, Caucasian, Asian, Indian, other.

Family History: genetic background, occupation.

\subsection{Hormonal stimulation for IVF}

The hormonal stimulation of women undergoing IVF treatment was carried out according to Fertility Associate's stimulation protocols: a) the "down regulation protocol (5.85)" and b) the "micro-dose flare protocol (5.9)". These protocols are based on the two most commonlyused hyper-stimulation methods in the world. In the period of 2007-2010 at Fertility Associates Wellington $\sim 60 \%$ and $\sim 30 \%$ of women underwent stimulation using the "5.85" and "5.9" protocol respectively. In 2011, about 44 and $40 \%$ of women underwent IVF procedures with the use the down-regulation protocol and the micro-dose flare protocol, respectively.

The long agonist protocol (“5.85") used was based on that described by Damario et al. (1997). This protocol employs pre-treatment with an oral contraceptive pill (Levlen® ED or Microgynon ${ }^{\circledR} 30$ ED, Bayer HealthCare, Auckland, New Zealand) for 25 days followed by the administration of a GnRH agonist, which is overlapped with the final 5 days of oral contraceptive. Patients begin taking the OCP following onset of a spontaneous menstrual period or progestin-induced withdrawal bleed. The Fertility Associate's long protocol recommends the pre-treatment with OCP for at least 21 days, where GnRH agonist (Buserelin, Suprafact, Sanofi-Aventis, Paris, France) treatment (400 $\mu \mathrm{g}$ subcutaneous/day) starts from the $15^{\text {th }}$ day of the OCP, and overlaps the OCP treatment for 7 days. Gonadotrophin treatment (rFSH, Puregon, Merck \& Co./MSD, New Jersey, USA) is initiated 
in daily doses (75-300 IU, depending on the woman and her predicted ovarian response) after 14 days of GnRH agonist. Monitoring of serum oestradiol levels and follicular development by ultrasound commence on day 7 of gonadotrophin treatment. The timing of hCG administration was based on the extent of follicular development and serum oestradiol levels. Ovulation was initiated using choriogonadotrophin alpha $(250 \mu \mathrm{g}$ Ovidrel, Merck KGaA, Darmstadt, Germany) when at least three follicles had reached $\geq 18 \mathrm{~mm}$ diameter, or after two follicles had reached $\geq 20 \mathrm{~mm}$ (Appendix 2.1).

The "micro-dose flare" protocol ("5.9") was based on that described by Scott and Navot (1994) (Appendix 2.2). The "5.9" protocol employs pre-treatment with OCP for 21 to 28 days, followed by the subcutaneous administration of $50 \mu \mathrm{g}$ GnRH agonist (Buserelin) every 12 hours for three days after discontinuing the OCP. On the third day of administration of GnRH agonist, ovarian stimulation is augmented with 225-300IU exogenous rFSH. Monitoring of serum oestradiol levels and follicular development by ultrasound commenced on Day 4 or Day 6 depending on the daily dose of rFSH stimulation (225IU or 300IU, respectively). The initiation of ovulation was done similarly to that in the long protocol, when at least three follicles had reached $\geq 18 \mathrm{~mm}$ diameter, or after two follicles had reached $\geq 20$ mm (Appendix 2.2).

\subsection{Embryological manipulations and CC collection}

Oocytes and embryos were handled and cultured according to Fertility Associate's protocols (Appendix 3.1-3.3), with the exception that throughout the entire in vitro culture, oocytes and embryos were cultured individually.

In brief, at 36 hours following the administration of an ovulation trigger (i.e. hCG; $250 \mathrm{IU}$, Ovidrel, Merck KGaA, Darmstadt, Germany), the women underwent ultrasound guided transvaginal oocyte retrieval. Follicular fluid containing the COC was aspirated and collected into $14 \mathrm{ml}$ tubes (Cat. No. 352057, BD Falcon, NJ, USA), before being transferred to IVF culture dishes (Cat. No. 353652, BD Falcon) incubating on a heated stage $\left(37^{\circ} \mathrm{C}\right)$ of a stereo microscope. The COC were recovered into 1-well IVF culture dishes (Cat. No. 353653, BD Falcon) containing $1 \mathrm{ml}$ G-MOPS Plus media (Vitrolife, Göteborg, Sweden) and rinsed. Following rinsing in G-MOPS Plus media, COC were transferred to individual culture wells 
(Cat. No. 144444, Nalge Nunc International, Rochester, NY, USA) containing 0.5 ml G-IVF Plus culture media (Vitrolife) under paraffin oil (OVOIL, Vitrolife) until denudation.

At 38 hours post-hCG treatment, the morphological characteristics of the CC were recorded (Appendix 4). Following scoring, the individual COC were exposed to $0.5 \mathrm{ml}$ (10 IU) of hyaluronidase solution (HYASE-10x in G-Mops Plus media, Vitrolife) in 4-well dishes (\#144444, Nunc) for several seconds before being transferred to $0.5 \mathrm{ml}$ G-MOPS Plus media. With the use of a denudation pipette (Cat. No. 14302, Vitrolife), the CC were mechanically dissociated from the oocyte (Appendix 3.1). Each denuded oocyte was then transferred into individual $15 \mu \mathrm{L}$ culture drops (G-IVF Plus) under paraffin oil (OVOIL) in IVF culture dishes (BD Falcon). After denudation, the morphological characteristics of the oocyte were recorded (Appendix 4).

Both residual solutions (G-MOPS Plus media and HYASE solution) containing the dissociated CC for each individual oocyte were pooled in a $1.5 \mathrm{ml}$ tube (\#2230-00, SSI, Lodi, CA 95240 USA) and centrifuged at $894 g$ for 1 minute. Most of the supernatant was removed and the pellet containing the CC was snap-frozen in liquid nitrogen for total RNA extraction.

The oocytes were subjected to intra-cytoplasmic sperm injection (ICSI) at 39 hours post-hCG treatment (Appendix 3.1) and cultured individually in a $15 \mu \mathrm{L}$ micro-drop culture media under paraffin oil (OVOIL) in IVF culture dishes (\#353652, BD Falcon). Embryos were cultured in sequential culture media (G5 Series, Vitrolife) in $37^{\circ} \mathrm{C}$ incubators (MINC; COOK, Brisbane, Australia) under $5 \% \mathrm{O}_{2}$ and $6 \% \mathrm{CO}_{2}$ culture conditions according to both the manufacturer's and Fertility Associate's protocols. Embryo transfer was carried out according to Fertility Associate's embryo transfer protocol. In brief, embryos selected for transfer were first moved into a designated transfer dish (Cat. No. 353653, BD) in $50 \mu 1$ Embryo Glue media (Vitrolife) under paraffin oil (Vitrolife). Embryos were then aspirated with $\sim 20 \mu \mathrm{l}$ transfer media into embryo transfer catheters (G20683, Sydney IVF catheter, COOK, Brisbane, Australia or Labotect, Labor-Technik-Göttingen GmbH, Göttingen, Germany) and were handed to the clinician, who introduced the catheter content (fluid with embryo) into the lower third portion of the uterine cavity of the patient.

All manipulations (except ICSI) were performed with the aid of a stereo-microscope (SZ61, Olympus, Olympus Corporation, Shinjuku, Tokyo, Japan) fitted with a heated stage set at 37 
${ }^{\circ} \mathrm{C}$. The ICSI procedure was performed using an inverted microscope at $37{ }^{\circ} \mathrm{C}$ (TE300, Nikon, Tokyo, Japan). Manipulations of COC were carried out using sterile Pasteur pipettes (Cat. No. PP-5.75-90, Charlottesville, VA, USA) and denuded oocytes and embryos were handled with micro-pipettes (Cat. No. 40270570, LabServ, Thermo Fisher Scientific, Waltham, MA, USA) fitted with sterile tips (Cat. No. T-200-C-R-S, Axygen, Union City, CA, USA).

The morphological appearances of COC, oocytes and embryos were recorded from the day of COC collection (Day 0) until Day 6 of embryo culture. As previously mentioned, the CC from each COC was graded according to morphological appearance under a stereomicroscope. For statistical purposes, COC graded as 0 and 1 were classified "light', where 0 represents a light shaded CC mass with no abnormalities and 1 represents a light shaded small CC mass ("ball like"). Those COC graded as 2 and 3 were classified as "dark", where grade 2 stands for dark shaded CC masses and 3 stands for a CC mass with a light shaded base incorporating large dark patches. Oocytes were classified according to meiotic stage; i.e. GV, MI, MII or abnormal (Appendix 4). Morphologically, germinal vesicle (GV) oocytes are characterised by the presence of the nucleus with a single large nucleolus (germinal vesicle), eccentrically located in the ooplasm. Methaphase I (MI) oocytes are those oocytes that have undergone germinal vesicle breakdown (display no GV) and display no polar body. Methaphase II (MII oocytes) are characterised by the presence of the first polar body in the perliviteline space (Mandelbaum 2000). Embryos were assessed at 18 hours post-ICSI for fertilization, 25 hours for early cleavage, 42 and 66 hours for cleavage stage and 114 and 138 hours for blastocyst development (Appendix 3.2 and Appendix 3.3). Cleavage-stage embryos were graded according to widely used selection criteria such as cell number and morphological appearance (Cummins et al. 1986; Sakkas et al. 1998). Embryos were considered top quality if they presented early cleavage, divided to 4 cells on day 2 and reached at least 7 cells on day 3 of in vitro culture. Blastocyst development was assessed on Day 5 and Day 6 of culture. Blastocysts were given a numerical score from 1 to 6 representing their degree of expansion and hatching status: (1) early blastocyst with the blastocoel being less than half the volume of the embryo; (2) blastocyst with the blastocoel being greater than or equal to half of the volume of the embryo; (3) full blastocyst with the blastocoel completely filling the embryo; (4) expanded blastocyst with the blastocoel volume being larger than that of the early embryo and the zona is thinning; (5) hatching blastocyst with the trophectoderm starting to herniate though the zona and; (6) hatched blastocyst where 
the blastocyst has completely escaped from the zona. Furthermore, blastocysts were graded according to the morphological appearance of their inner cell mass (ICM): (A) tightly packed with many cells; (B) loosely grouped with several cells and; (C) very few cells. Similarly, the trophectoderm was graded as: (A) many cells forming a tightly knit epithelium; (B) few cells and; (C) very few cells forming a loose epithelium (Gardner and Sakkas 2003). Blastocysts were selected for transfer or freezing if they were graded at least 1 for expansion, B for ICM and B for trophectoderm.

\subsection{Pregnancy monitoring and live birth follow-up}

The recording of pregnancies and live births was carried out according to Fertility Associate's and the Australia and New Zealand Assisted Reproduction Database (ANZARD) requirements (http://www.preru.unsw.edu.au/preruweb.nsf/page/ANZARD).

In brief, pregnancy was determined initially from plasma hCG and progesterone levels on Day 14 after egg collection followed by a second measurement of these parameters on Day 18. A positive pregnancy was recorded if the hCG level at Day 14 was $\geq 18 \mathrm{iu} / 1$. Depending on the result of Day 18 progesterone levels, luteal support was stopped, reduced or continued until $\sim$ Week 8, when the presence of yolk sac and foetal heart beat (FHB) was measured by ultrasound scan. Positive implantation was considered if a FHB was present. Following birth, the gestation period, weight and gender of the live born baby was recorded for this study.

\subsection{Cell count and viability index for cumulus cells after OPU}

Following collection and re-suspension of single CC masses in $50 \mu$ l of G-MOPS Plus culture media, total CC numbers were counted using a haemocytometer under an inverted microscope at 400x magnifications. The total number of CC in the cell suspension (as an indication of CC mass) was calculated according to the formula:

Total number of CC in one COC $=\operatorname{Navg} \mathbf{x}$ DF $\mathbf{x} \mathrm{SV}$

Key: Navg = average number of cells in one large square of the haemocytometer $\mathrm{DF}=$ dilution factor (if suspension was further diluted)

$\mathrm{SV}=$ cell suspension volume $(50 \mu \mathrm{l})$ 
For assessment of cell viability, an equal amount of $0.4 \%$ Trypan blue stain solution (Ajax Finechem Pty Ltd, Auckland, New Zealand) was added to the cell suspension. Dead cells incorporate Trypan blue and appear blue under the microscope, whilst intact (viable) cells are able to exclude Trypan blue and remain transparent. Cell viability was calculated according to the formula:

$\%$ viable cells $=\mathrm{N}$ viable cells $/$ Total $\mathrm{N}$ of cells $\mathbf{x} 100$

\subsection{RNA Extraction and cDNA Synthesis}

Total RNA was extracted from individual CC masses using the ArrayPure ${ }^{\mathrm{TM}}$ Nano-scale RNA purification kit (EPICENTRE Biotechnologies, Cat. No. MPS04050, Wisconsin, USA) and stock solutions of $70 \%$ ethanol and isopropanol (Global Science, Cat. No. APPA39281000PE, New Zealand). For the preparation of $70 \%$ ethanol, $100 \%$ analytical grade ethanol (Sigma-Aldrich, Cat. No. 24106) was diluted to $70 \%$ in Diethylpyrocarbonate (DEPC) water and was stored at room temperature. DEPC water was prepared according to the formula: $1 \mathrm{ml}$ of DEPC (Sigma-Aldrich, Cat. No. 40718) was dissolved in 1L of distilled water and stirred overnight in the fume hood and sterilized under standard autoclave conditions. The RNA purification procedure involved adding $30 \mu \mathrm{l}$ of nano-scale lysis solution containing $1 \mu$ l Proteinase $\mathrm{K}$ to the tube containing the captured cell pellet. The contents were mixed thoroughly by vortexing followed by 15 minutes incubation at $65^{\circ} \mathrm{C}$. Thereafter, the samples were placed on ice for five minutes, followed by the addition of $18 \mu 1$ of $5 \mathrm{M}$ ammonium acetate solution (MPC Protein Precipitation Reagent). In the next step, the lysed samples were vortexed vigorously, and centrifuged for seven minutes at $12,000 \mathrm{~g}$ to pellet the debris. The supernatant was transferred to a clean microcentrifuge tube and total RNA was precipitated by the addition of $50 \mu 1$ of isopropanol, mixing by vortexing and then pelleting by centrifugation at $4^{\circ} \mathrm{C}$ for five minutes at $12,000 \mathrm{~g}$. Following the removal of the residual isopropanol and air drying the pellet, contaminating DNA was removed by resuspending the pellet in $20 \mu \mathrm{l}$ DNase solution (prepared by the addition of $0.5 \mu 1$ of RNaseFree DNase I to $20 \mu \mathrm{l}$ of $1 \mathrm{X}$ DNase Buffer). The tubes containing the samples were incubated at $37^{\circ} \mathrm{C}$ for 30 minutes. In the next step, $20 \mu \mathrm{l}$ of $2 \mathrm{X}$ nano-scale lysis solution was added to the samples and mixed by vortexing. Contaminating proteins were removed again by treating the samples with $20 \mu \mathrm{l}$ of MPC Protein Precipitation Reagent, vortexing, cooling (five 
minutes on ice) and centrifuging at $4^{\circ} \mathrm{C}$ for five minutes at $12,000 \mathrm{~g}$. Following the transfer of the supernatant to a clean microcentrifuge tube, total RNA was precipitated by the addition of $50 \mu \mathrm{l}$ of isopropanol and pelleted by centrifugation at $4^{\circ} \mathrm{C}$ for five minutes at $12,000 \mathrm{~g}$. The isopropanol was aspirated and the RNA pellet was rinsed with $70 \%$ ethanol. After a final centrifugation step and aspiration of the ethanol, the pellet was air dried and re-suspended in $10 \mu \mathrm{l}$ of ultra-pure $\mathrm{H}_{2} \mathrm{O}$. Total RNA samples were either stored at $-80^{\circ} \mathrm{C}$ until further use, or used immediately for cDNA synthesis.

Following extraction, the total RNA samples were reverse-transcribed to cDNA using the SuperScript ${ }^{\circledR}$ VILO $^{\text {TM }}$ cDNA Synthesis Kit (Cat. No. 11754-050, Invitrogen, CA, USA). This kit provides the high-temperature capability of SuperScript ${ }^{\circledR}$ III Reverse Transcriptase in an optimized format for generating first-strand cDNA for use in QPCR. This formulation is reported to enhance the efficiency of cDNA synthesis and can be used with very low to very high amounts of total RNA (up to $2.5 \mu \mathrm{g}$ total RNA in a $20-\mu 1$ reaction), giving a linear response in message abundance as measured by QPCR.

For a single reaction, the following components were combined in a tube on ice: $4 \mu \mathrm{l}$ of $5 \mathrm{x}$ VILO $^{\mathrm{TM}}$ reaction Mix, $2 \mu \mathrm{l}$ of $10 \mathrm{x}$ SuperScript ${ }^{\circledR}$ enzyme Mix, $10 \mu \mathrm{l}$ of RNA (up to $2.5 \mu \mathrm{g}$ ) and $4 \mu \mathrm{l}$ of ultra-pure $\mathrm{H}_{2} \mathrm{O}$. The contents of tube were mixed by brief vortexing. Thereafter, the samples were incubated for ten minutes at $25^{\circ} \mathrm{C}$, one hour at $42{ }^{\circ} \mathrm{C}$ and five minutes at 85 ${ }^{\circ} \mathrm{C}$. Following the incubation, the cDNA samples were stored at $-80^{\circ} \mathrm{C}$ until QPCR analysis.

\subsection{Quantitative PCR (QPCR)}

For primer optimization and validation of the housekeeper gene, QPCR using SYBR green chemistry was used. For probe optimization and quantification of candidate/housekeeper genes, QPCR using TaqMan chemistry was used (see page 54 for more details). The reaction kits for both methods came from Agilent Technologies, USA (Cat. No. 600548 and 600553 , respectively).

\subsubsection{SYBR Green Method}

This method uses a DNA dye, SYBR® Green I, which in an unbound state exhibits little fluorescence. However, when SYBR® Green I binds to double-stranded (ds) DNA, it 
exhibits significantly increased fluorescence signal. Thus during the denaturation step when all DNA becomes single-stranded, the dye produces little fluorescence. In contrast, during the annealing step when primers attach to the target sequence and dsDNA is synthesized, the SYBR Green I dye binds to the dsDNA and exhibits a strong fluorescent signal. This fluorescence signal is then monitored in a linear fashion as the PCR product is amplified over a range of PCR cycles. Therefore the cycle at which the intensity of the fluorescence signal becomes significantly higher than the background (i.e. cycle threshold, $\mathrm{C}_{\mathrm{T}}$ ), will depend on the initial concentration of the gene target present (Brilliant SYBR® Green QPCR, Instruction Manual, Agilent Technologies).

\subsubsection{Multiplex (TaqMan) Method}

The multiplex QPCR method allows multiple genes to be amplified simultaneously in the same reaction vessel. This method utilizes a probe-based chemistry in which each probe has a spectrally different fluorophore and quencher attached. The TaqMan probes used in this reaction are linear, with the fluorophore usually at the 5' end of the probe and the quencher is either internal or at the 3 ' end. In the intact form (either hybridized or not with the target) the probe exhibits no fluorescent signal. However, in the annealing step after the probe hybridizes to the target, the DNA polymerase separates the fluorophore from the quencher resulting in the emittance of fluorescence. As with the SYBR green chemistry, this fluorescence signal is then monitored in a linear fashion as the PCR product is amplified over a range of PCR cycles. Therefore the cycle at which the intensity of the fluorescence signal becomes significantly higher than the background (i.e. $\mathrm{C}_{\mathrm{T}}$ ), will depend on the initial concentration of the gene target present (Brilliant Multiplex QPCR Master Mix, Instruction Manual, Agilent Technologies).

\subsubsection{Fluorescence Monitoring in Real-Time}

The change of fluorescence signal is monitored in real-time over the course of the PCR reaction. The results are displayed as an amplification plot. This information is then used to quantify the initial target copy number, which is calculated from values provided by a threshold cycle $\left(\mathrm{C}_{\mathrm{T}}\right) . \mathrm{C}_{\mathrm{T}}$ is defined as the cycle at which fluorescence is determined to be statistically significant above background, and it is inversely proportional to the log of initial copy number. The more template that is initially present, the fewer the number of cycles it 
takes to reach the point where the fluorescence signal is detectable above background. Quantification based on $\mathrm{C}_{\mathrm{T}}$ has the advantage over endpoint quantification in that measurements are taken during the exponential phase of the PCR amplification, when the reaction efficiency is not yet influenced by limiting reagents. The more template that is initially present in the reaction, the lower the $\mathrm{C}_{\mathrm{T}}$ value (Brilliant Multiplex QPCR Master Mix - Instruction Manual).

\subsubsection{Primers and Probes}

All primers and TaqMan probes (Table 2A) were designed using the computer package 'Beacon Designer' (Premier Biosoft International, CA, USA) based on sequences obtained through NCBI and were obtained from Invitrogen and Sigma-Proligo (TaqMan probes;

Proligo-France SAS 1, Paris, France or Proligo-Singapore Pte Ltd, Helios, Singapore), respectively.

Table 2A: Nucleotide (nt) sequences for primers and TaqMan probes for QPCR including the size of the resultant amplicon in regards to number of nt for human ALCAM (NM_001627.3), SFRP2 (NM_003013.2), NRP1 (NM_003873.5), PR (NM_001202474.1), VCAN (NM_004385.4), HAS2 (NM_005328), FSHR (NM_000145), GLUT4 (NM_001042), RPL19 (NM_000981) and 18S (NR_003286) mRNA. F=5'; R=3'.

\begin{tabular}{|c|c|c|c|}
\hline Gene & Taqman probe (5'-3') & Primers (5'-3') & $\begin{array}{l}\text { Size } \\
(n t)\end{array}$ \\
\hline ALCAM & (HEX) TTCCTGCCGTCTGCTCTTCTGCCTCTT (BHQ1) & $\begin{array}{l}\text { F-CACCAAGAAGGAGGAGGAATATG } \\
\text { R-GCAAGGTATGATAATGGTATCTCCA }\end{array}$ & 149 \\
\hline SFRP2 & (ROX)AAACAACAAACAACAACCAACCAGACCCAAGT(BHQ2) & $\begin{array}{l}\text { F- GCCCACCCGAATCTTGTAGAAA } \\
\text { R-CAACCTCAGTGGGAAGTGAAAATC }\end{array}$ & 173 \\
\hline NRP1 & (ROX)CATCTTCCAGTCCGAGCCGTTGTTG(BHQ2) & $\begin{array}{l}\text { F- GCACCTCATTCCTACATCA } \\
\text { R- CTTGCGTTTGCTGTCATC }\end{array}$ & 183 \\
\hline PR & (HEX)CACTTTCTTCTCCCATGCTTTATCTCCATCAAT (BHQ1) & $\begin{array}{l}\text { F- GTGGTCAATAGTGTTTGCTG } \\
\text { R-GTAGGAAGCAAAGGTGGAA }\end{array}$ & 193 \\
\hline VCAN & (6FAM)TCCCATTCGCAGCCTTTAGCATCAT(BHQ1) & $\begin{array}{l}\text { F- ATGGCTTTTCCTGGACAG } \\
\text { R- GGAGTCCTTAGTTCCATCAG }\end{array}$ & 130 \\
\hline HAS2 & (HEX)AACTGCCCGCCACCGACCCTCC(BHQ1) & $\begin{array}{l}\text { F-GGTCGTCTCAAATTCATCTGATCTC } \\
\text { R-GGATACATAGAAACCTCTCACAATGC }\end{array}$ & 145 \\
\hline FSHR & (6FAM)TCTGCTGTAGCTGGACTCATTGTCTTCTGCC(BHQ1) & $\begin{array}{l}\text { F-AAGTTGATTATATGACTCAGGCTAGG } \\
\text { R-AACTCAGTGTACGTCATGTCAAATC }\end{array}$ & 100 \\
\hline GLUT4 & (ROX)CCCGCCCTCGCACGTCACTCCG(BHQ2) & $\begin{array}{l}\text { F-TCTCCGGGTCCTTGGCTTG } \\
\text { R-GCGAAGATGAAAGAACCGATCC }\end{array}$ & 137 \\
\hline RPL19 & (CY3)CCAATGCCAACTCCCGTCAGCAGATCCG(BHQ3) & $\begin{array}{l}\text { F-GACCCCAATGAGACCAATGAAATC } \\
\text { R-GGAATGGACCGTCACAGGCTTG }\end{array}$ & 105 \\
\hline $18 \mathrm{~S}$ & & $\begin{array}{l}\text { F-GCCGCTAGAGGTGAAATTCTTG } \\
\text { R-CGGAACTACGACGGTATCTGATC }\end{array}$ & 128 \\
\hline
\end{tabular}


Optimum primer and probe concentrations were obtained by titrating concentrations of forward and reverse primers and TaqMan probes from 50nM to $300 \mathrm{nM}$. The optimal concentrations were deemed to be that which gave the lowest $C_{T}$ value. The optimum primer and probe concentrations are shown in Table 2B.

Table 2B: Final concentrations of primers and TaqMan probes in QPCR reactions for human ALCAM, SFRP2, NRP1, PR, VCAN, HAS2, FSHR, GLUT4, RPL19 and $18 S$.

\begin{tabular}{lccc}
\hline & \multicolumn{2}{c}{ Primers $(\mathbf{n M})$} & $\begin{array}{c}\text { Taqman } \\
\text { probe }\end{array}$ \\
\hline Gene & Forward & Reverse & (nM) \\
\hline ALCAM & 300 & 200 & 50 \\
SFRP2 & 200 & 300 & 50 \\
NRP1 & 200 & 300 & 100 \\
PR & 300 & 300 & 50 \\
VCAN & 200 & 300 & 50 \\
HAS2 & 300 & 200 & 50 \\
FSHR & 100 & 100 & 50 \\
GLUT4 & 100 & 100 & 100 \\
RPL19 & 100 & 100 & 50 \\
ISS & 300 & 200 & - \\
\hline
\end{tabular}

\subsubsection{QPCR Analysis}

The Corbett Rotor-Gene 6000 Series Software 1.7 was used to analyze all QPCR results. The optimal amount of cDNA template per QPCR reaction was deemed to be that resulted in a $C_{T}$ value within the measurable range (15-29), therefore $1.04 \mu \mathrm{l}$ of original template was used for each reaction.

Quantification of gene mRNA levels using the SYBR Green method was done according to manufacturer's instructions. In brief, for each cDNA sample, a singleplex reaction mix was prepared in $0.2 \mathrm{~mL}$ microtubes containing forward and reverse primers at optimized concentrations (Table 2B), $26 \mu 1$ of 2 x Brilliant $^{\circledR}$ Green QPCR Master Mix (Cat. No. 600548, Agilent Technologies CA, USA), $1.04 \mu \mathrm{l}$ of cDNA template and an appropriate aliquot of Ultra-Pure $\mathrm{H}_{2} \mathrm{O}$ (Invitrogen) to a total volume of $52 \mu$ l. After thorough vortexing, aliquots of $25 \mu \mathrm{l}$ were transferred in duplicate to $0.1 \mathrm{ml}$ strip-tubes and capped (Corbett Research; NSW, Australia 2137). The amplification reaction was run using a Rotor-Gene ${ }^{\mathrm{TM}} 6000$ Rotary 
Analyzer (Corbett Research) under the following conditions: 1 cycle of $95^{\circ} \mathrm{C}$ for $10 \mathrm{~min} ; 40$ cycles of $95^{\circ} \mathrm{C}$ for $15 \mathrm{sec}$ and $58-60^{\circ} \mathrm{C}$ (depending on the gene) for $60 \mathrm{sec}$.

Quantification of mRNA levels using the quadriplex TaqMan method was undertaken according to the manufacturer's protocols. In brief, the reaction mixes were prepared containing forward and reverse primers and TaqMan probes for one housekeeping (RPL19) and up to three genes of interest at optimized concentrations (Table 2B), $26 \mu \mathrm{l}$ of $2 \mathrm{x}$ Brilliant $^{\circledR}$ Multiplex QPCR Master Mix (Cat. No. 600553, Agilent Technologies), $1.04 \mu 1$ of neat cDNA and an aliquot of Ultra-Pure $\mathrm{H}_{2} \mathrm{O}$ to a total volume to $52 \mu \mathrm{l}$. After vortexing, aliquots of $25 \mu \mathrm{l}$ were transferred in duplicate to $0.1 \mathrm{ml}$ strip-tubes and capped. The amplification reaction was run using the Rotor-Gene ${ }^{\mathrm{TM}} 6000$ Rotary Analyzer under the conditions described above.

Controls included samples that underwent reverse transcription PCR with the exclusion of Superscript III/RNaseout enzyme mix to check the effectiveness of DNase treatment, and reactions that omitted addition of the template.

\subsubsection{Confirmation of genes by DNA sequencing}

Genes of interest were amplified by SYBR Green QPCR using the method described above. Thereafter, the amplification product was purified using the PureLink ${ }^{\mathrm{TM}}$ PCR Micro kit (Cat. No. K310010, Life Technologies Corporation, NY, USA) or the MinElute PCR purification kit (Cat. No. 28704, Qiagen, Hilden, Germany), following the manufacturer's protocols.

In short, the QPCR product was treated with a 4-5x volume of binding buffer and vortexed thoroughly. The entire volume was transferred to a column containing a silica membrane for binding the DNA, and the column was placed in a collection tube, to collect the eluate. The sample was then centrifuged for one minute at $10,000 \mathrm{~g}$ and the eluate was discarded. Thereafter, the column was treated with 650-750 $\mu$ l wash buffer and the tube was centrifuged again for one minute at 10,000 $\mathrm{g}$. Again, the eluate was discarded and the column was centrifuged again to remove any residual washing buffer. Subsequently, the column was moved to a new collection tube and $10 \mu \mathrm{l}$ elution buffer or ultra-pure $\mathrm{H}_{2} \mathrm{O}$ was added to the silica membrane of the column. Thereafter, the sample was incubated for 1 minute at room 
temperature and centrifuged for one minute at $10,000 \mathrm{~g}$ to collect the purified DNA into the elution tube. Samples were stored at $-80^{\circ} \mathrm{C}$ until further use.

After purification, the purified QPCR product was sent for sequencing to the Waikato DNA Sequencing Facility (Department of Biological Sciences, University of Waikato, Hamilton, New Zealand).

Results were matched against gene sequences provided by NCBI and in the case of a positive match, genes were confirmed.

\subsubsection{Validation of the QPCR analysis}

After the conditions for the QPCR reactions were optimized, the amplification efficiency for each gene was tested. Serial dilutions (1:1-1:32) of at least two samples were made to validate the amplification efficiency for each gene. A reaction efficiency of $\geq 80 \%$ was considered satisfactory (the reaction efficiencies for each gene are listed in Table 2C). This included the calculation of the line of best fit (slope \pm 0.1 ) for each gene when $C_{T}$ values were plotted against the log of input RNA or the log of total cell number in each CC mass. Additionally, $\mathrm{C}_{\mathrm{T}}$ values $(\leq 0.4$ cycles different) for identical samples for all mRNA transcripts were compared between results from singleplex and multiplex reactions.

Table 2C: Table of QPCR efficiency for each candidate gene in multiplex reactions.

\begin{tabular}{llc}
\hline & Gene & Efficiency (\%) \\
\hline Reaction 1 & HAS2 & 83 \\
& FSHR & 91 \\
& GLUT4 & 87 \\
& RPL19 & 93 \\
\hline Reaction 2 & ALCAM & 91 \\
& SFRP2 & 88 \\
& RPL19 & 93 \\
\hline Reaction 3 & NRP1 & 80 \\
& PR & 80 \\
& VCAN & 84 \\
& RPL19 & 81
\end{tabular}




\subsubsection{Quantification of gene expression by multiplex QPCR}

Expression levels of the candidate genes in each sample were calculated by the $2^{-\left(\Delta \Delta \mathrm{C}_{\mathrm{T}}\right)}$ method (Livak and Schmittgen 2001). Expression levels were standardised against a calibrator sample and normalized with respect to the house-keeping gene, RPL19. Results are therefore presented as fold-changes. Thus for each CC-specific gene, the $\mathrm{C}_{\mathrm{T}}$ value for $R P L 19$ expression for each sample was subtracted from the $\mathrm{C}_{\mathrm{T}}$ value for the gene of interest resulting in a $\Delta \mathrm{C}_{\mathrm{T}}$ value. Then the calibrator's $\Delta \mathrm{C}_{\mathrm{T}}$ value was subtracted from the $\Delta \mathrm{C}_{\mathrm{T}}$ value of a specific CC sample resulting in a $\Delta \Delta \mathrm{C}_{\mathrm{T}}$ value. To convert these values to linear volumes (i.e. fold change) for analyses, the following equation was performed: $2^{-(\Delta \Delta C}$ )

The standard errors of the mean (SEM) were calculated by the formula $\left.2^{-(\Delta \Delta C}{ }_{T}^{ \pm S E M}\right)$.

The ratio of mRNA expression between genes was calculated using the formula, $2^{-\left(1 \text { st gene } \mathrm{C}_{\mathrm{T}}\right.}{ }^{-}$ 2nd gene $\mathrm{C}_{\mathrm{T}}$, resulting in a relative fold difference. Correlations between two genes of interest were undertaken using 2-tailed Pearson's R test and the SPSS statistical package (PASW Statistics 18, IBM, New York, USA). 


\section{Chapter 3 VALIDATION OF RPL19 AS A HOUSEKEEPING GENE FOR HUMAN CUMULUS CELLS}

\subsection{Introduction}

Quantitative PCR is an invaluable technique used for the measurement and comparison of target genes in biological samples. Moreover, in small biological samples and with respect to genes expressed with low abundances, QPCR represents the only technique sensitive enough to measure mRNA expression reliably (Huggett et al. 2005). The accurate measurement of target mRNAs and normalization across data sets is strongly influenced by the choice of reference genes (housekeeping genes). In gene expression analyses, the abundance of the target transcript is normalized against the housekeeping gene, whilst comparing multiple samples is done by comparing the ratios of normalized expression levels for the gene of interest in each sample against a constant sample (calibrator). Ideally, the expression of the housekeeping gene should be constant in all samples and independent of different experimental conditions (i.e. cause of ovarian infertility in women undergoing IVF) (Livak and Schmittgen 2001; Quackenbush 2002; Dheda et al. 2004). In the process of normalization of the target genes with respect to the housekeeping gene, it is important to assess the integrity and quality of cells that are subjected to analysis (Huggett et al. 2005). The negative effects of cell apoptosis on DNA integrity and the precision of gene expression analysis are well documented in post-mortem tissue studies (Koppelkamm et al. 2011). In this context it is useful to know that the selected housekeeping gene correlates to the cell numbers from the specific sample (Huggett et al. 2005).

In recent years, many papers have been published where classical housekeeping genes were chosen without appropriate validation. Reports that have investigated the stability of housekeeping genes in multiple tissues and samples have indicated significant variability in the expression of some putative housekeeping genes suggesting that great care is needed when selecting a reference gene for a tissue/cell-specific study (Chari et al. 2010). Indeed, it has been proposed that authors need to demonstrate the suitability of the reference gene of choice for the experiment in question (Huggett et al. 2005; Chari et al. 2010; Fu et al. 2010; Frota et al. 2011). The majority of the studies investigating gene expression levels in human CC samples have used ribosomal 18S RNA or glyceraldehyde-3-phosphate dehydrogenase $(G A P D H)$ as the housekeeping gene (Hasegawa et al. 2005; Zhang et al. 2005; Feuerstein et 
al. 2007; Hamel et al. 2008; van Montfoort et al. 2008; Anderson et al. 2009; Gebhardt et al. 2011). Researchers investigating the housekeeping properties of GAPDH in ovarian samples have reported its high variability, and inappropriateness as a reference gene in some instances (Fu et al. 2010; Frota et al. 2011). Ribosomal 18S RNA is recognised widely as a good housekeeping gene, however its abundance is one of the highest amongst the housekeeping genes, making it unsuitable when studying genes expressed with low abundances (Feuerstein et al. 2007; Fu et al. 2010). Moreover, $18 S$ RNA is reported to have low expression stability in human ovarian cancer and in caprine ovaries (Fu et al. 2010; Frota et al. 2011). On the other hand, the 60S ribosomal protein L19 (RPL19) gene is expressed in a similar abundance to that of many of the target genes in CC and was one of the more stable housekeeping genes following studies in caprine ovaries and human lung tissues (Feuerstein et al. 2007; Chari et al. 2010; Frota et al. 2011). However, there appears to have been no studies that have investigated the relationship between the number of CC and RPL19 expression levels.

The aim of this study was to verify a) the quality of CC samples collected for mRNA extraction as measured by $\mathrm{CC}$ viability, and b) the housekeeping properties of RPL19 and $18 S$ in human CC samples.

\subsection{Materials and Methods}

Following the removal of the multiple layers of CC (i.e. the CC mass), the oocytes underwent IVF and embryological procedures as described in general methodology. Cell viability was determined from 59 isolated individual CC masses. CC numbers were determined from a further 21 individual CC masses. The numbers of CC in each CC mass were counted using a haemocytometer under an inverted microscope at 400x magnification, and the viability of the cells was assessed using a $0.4 \%$ (w/v) Trypan blue stain solution (Chapter 2). The 59 samples collected for the assessment of viability were used only for validation of the denuding method and were discarded after vibility assessment. For validation of the housekeeper gene RPL19, expression levels in a total of 21 individual CC masses from three women were compared to that of another common housekeeper gene $(18 S)$ and to CC number. CC masses were collected and re-suspended in $50 \mu \mathrm{l}$ of G-MOPS Plus media (Vitrolife). From this suspension, an aliquot of $20 \mu \mathrm{l}$ was used for determining total CC number per mass and the remaining $30 \mu \mathrm{l}$ of cell suspension were used for quantifying the expression levels of RPL19 and 18s mRNA. 
QPCR methods:

Total RNA was extracted and cDNA was synthesized using methods described in general methodology (see Chapter 2). Quantification of RPL19 and $18 S$ mRNA levels was undertaken using the Brilliant SYBR® Green QPCR Master Mix kit (Stratagene, La Jolla, CA, USA) and 2x Brilliant ${ }^{\circledR}$ Multiplex QPCR Master Mix kit (Stratagen) according to manufacturer's instructions and methods described in Chapter 2. In short, for each cDNA sample, a singleplex reaction mix was prepared in $0.2 \mathrm{~mL}$ microtubes each containing forward and reverse primers for $18 S$ or RPL19 at optimized concentrations (Table 2B), $26 \mu 1$ of 2x Brilliant ${ }^{\circledR}$ Green QPCR Master Mix, $1.04 \mu \mathrm{l}$ of diluted cDNA (1:50 for RPL19 and 1:500 dilution for 18S) and an appropriate aliquot of Ultra-Pure $\mathrm{H}_{2} \mathrm{O}$ (Invitrogen) to a total volume of $52 \mu \mathrm{l}$. After thorough vortexing, aliquots of $25 \mu \mathrm{l}$ were transferred, in duplicate, into $0.1 \mathrm{ml}$ tubes and capped (Corbett Research). The amplification reaction was run on a Rotor-GeneTM 6000 Rotary Analyzer (Corbett Research) using the following conditions: 1 cycle of $95^{\circ} \mathrm{C}$ for $10 \mathrm{~min} ; 40$ cycles of $95^{\circ} \mathrm{C}$ for $15 \mathrm{sec}$ and $60^{\circ} \mathrm{C}$ for $60 \mathrm{sec}$. For quantification of RPL19 mRNA levels by the TaqMan method, reaction mixes were prepared containing forward and reverse primers and TaqMan probes at optimised concentrations (Table 2B), 26 $\mu 1$ of 2x Brilliant ${ }^{\circledR}$ Multiplex QPCR Master Mix (Stratagen), $1.04 \mu 1$ of neat cDNA and an aliquot of Ultra-Pure $\mathrm{H} 2 \mathrm{O}$ to adjust the total volume to $52 \mu \mathrm{l}$. After vortexing, aliquots of 25 $\mu 1$ were transferred, in duplicate, to $0.1 \mathrm{ml}$ tubes and capped (Corbett Research). The amplification reaction was run on the Rotor-GeneTM 6000 as described above. Finally, RPL19 and $18 S$ cycle threshold $\left(\mathrm{C}_{\mathrm{T}}\right)$ values were plotted against log of total cell numbers in each associated CC mass.

\subsection{Results}

The viability of CC after denudation was $86.4 \pm 1.3 \%$ (mean \pm S.E.M; $\mathrm{N}=59$ ). Of the $21 \mathrm{CC}$ masses collected for QPCR validation, 20 had detectable levels of $18 S$ and RPL19 mRNA. The mean \pm SEM number of CC per mass (cells counted by haemocytometer) was $19680 \pm$ 2744 (range 2650 - 44900). These samples were only used for validation purposes. The relationship between CC numbers and expression levels of RPL19 and 18S are shown in Figure 3. The slopes and correlation efficiencies for the lines of best fit were similar for the $\mathrm{C}_{\mathrm{T}}$ values for $18 \mathrm{~S}$ using the SYBR green QPCR method, and for RPL19 using the SYBR green and TaqMan QPCR methods when plotted against the log number of CC. 


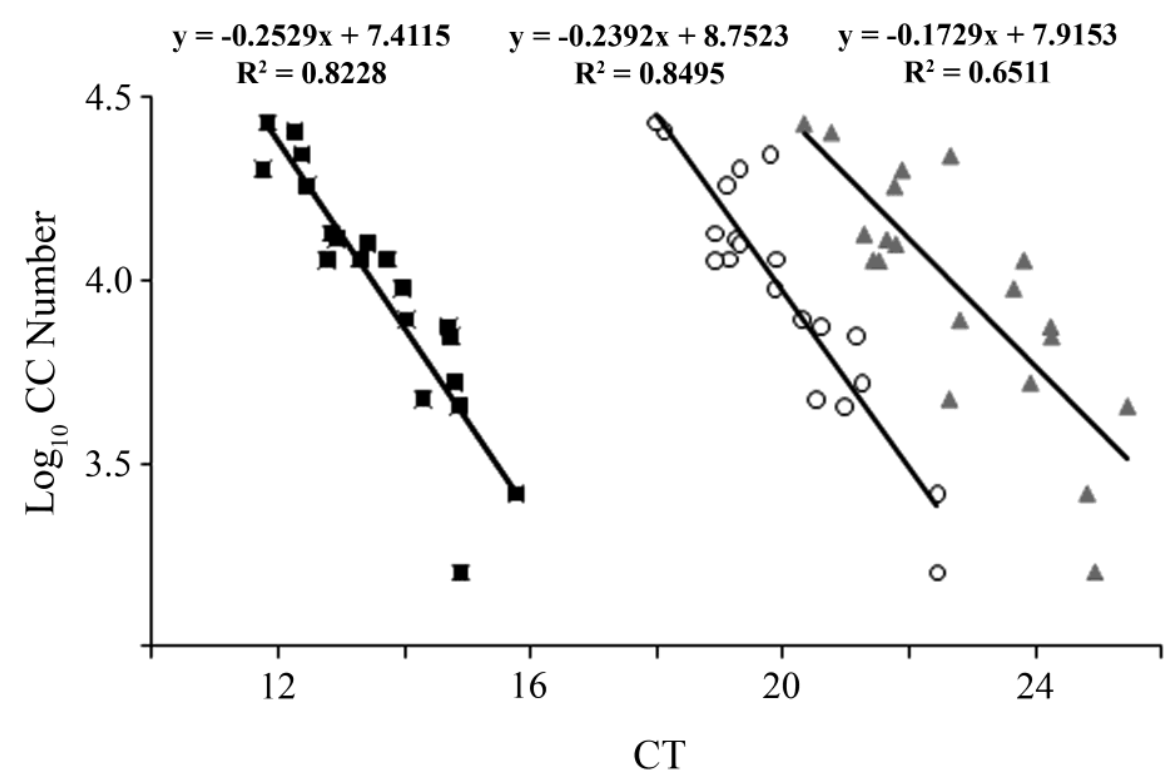

Figure 3: Linear regressions of $\log _{10}$ Cumulus Cell (CC) number versus Cycle Threshold $\left(\mathrm{C}_{\mathrm{T}}\right)$ value for the housekeeping genes RPL19 and 18S. Comparisons were made of $\log _{10} \mathrm{CC}$ number against 18S using SYBR Green QPCR (匹), RPL19 using TaqMan QPCR (०) and RPL19 using SYBR green QPCR ( $\mathbf{\Delta})$.

\subsection{Discussion}

The current study has provided the first evidence that RPL19 is an appropriate housekeeper gene for studies of gene expression in human CC. A strong positive correlation $(\mathrm{P}<0.0001)$ was found between RPL19 expression levels and the number of CC. Moreover, the regression equation of $\log \mathrm{CC}$ number $=-0.2392 *(R P L 19 \mathrm{Ct}$ value $)+8.7523$ (Figure 4$)$ that resulted from this preliminary study will permit the total number of $\mathrm{CC}$ in each $\mathrm{CC}$ mass collected to be estimated from RPL19 levels.

A previous report that investigated the feasibility of RPL19 as a housekeeping gene in CC collected from patients following IVF treatment found that the expression of RPL19 and 18S in $\mathrm{CC}$ were similar in relation to oocyte maturity, and that expression of $18 S$ was more abundant than that of RPL19 (Feuerstein et al. 2007). Indeed several studies have confirmed that many genes expressed in cumulus cells (i.e. GREM1, BDNF, TNFAIP6, PTX3, ALCAM, $S D C 4$ and $C A L M 2$ ) can have similar expression levels in $\mathrm{CC}$ from mature as well as immature oocytes (McKenzie et al. 2004; Anderson et al. 2009; Adriaenssens et al. 2010; 
Wathlet et al. 2011). Unlike previous studies, the expression of the RPL19 housekeeping gene in this study was compared to $\mathrm{CC}$ number, and a strong linear relationship was observed. Importantly, the results from this study indicate that it is possible to isolate CC of high viability, that provide an appropriate RNA template for the measurement of relative levels of candidate gene expression with respect to RPL19. 


\section{Chapter 4 OOCYTE DEVELOPMENTAL INDICATORS AND RELATIONSHIPS WITH CC NUMBERS}

\subsection{Introduction}

Previous studies that investigated the degree of expansion of the COC ( $\mathrm{Ng}$ et al. 1999; Rattanachaiyanont et al. 1999) and the incidence of apoptosis in CC (Lee et al. 2001; Host et al. 2002; Moffatt et al. 2002; Corn et al. 2005) in relation to oocyte quality, concluded that morphological evaluation of the COC provides limited prognosis on oocyte quality and is an unreliable marker in human IVF procedures.

However, there is limited information available on the total number of CC in a CC mass after ovulation, or after oocyte collection for human IVF. To the best of our knowledge, no study has investigated how CC numbers correlate with oocyte quality. The aims of the present study were to determine the numbers of $\mathrm{CC}$ in $\mathrm{COC}$ from each of the four experimental groups (Gp 1, <38yr old and healthy; Gp 2, <38yr old with PCOS; Gp 3, <38yr old with diminished ovarian reserve and; Gp 4, $\geq 40 \mathrm{yr}$ old and healthy) and to examine possible associations between $\mathrm{CC}$ number and indicators of oocyte development (embryo cleavage and pregnancy outcome).

\subsection{Materials and Methods}

In total, 68 women were investigated for embryological and pregnancy outcomes. They were classified into the four research groups described above: Group 1 (Gp 1, N=25); Gp 2 $(\mathrm{N}=11)$; Gp $3(\mathrm{~N}=12)$ and; Gp $4(\mathrm{~N}=20)$. Hormonal stimulation, oocyte collection, embryo culture and transfer and luteal support were all performed as described in the general methodology (Chapter 2). CC counts were performed as described in Chapter 2 and 3. CC numbers in single CC masses were estimated from RPL19 mRNA levels (see Chapter 3) using the following equation:

$\log \mathrm{CC}$ number $=-0.2392 *(R P L 19 \mathrm{Ct}$ value $)+8.7523($ Figure 3$)$. 


\section{Statistical analyses}

Indicators of oocyte development were established in accordance with internationally recognized embryological guidelines (ALPHA and ESHRE 2011), FAW protocols (Appendices 4.4 and 4.5) and with respect to recent reports to allow easy comparability between observations (Adriaenssens et al. 2010; Wathlet et al. 2011). Key embryological and pregnancy outcomes are reported as percentages. The comparisons of indicators of oocyte development between the four groups of women are represented as proportions and were analysed using the Chi-Square test and the SPSS statistical package (PASW Statistics 18, IBM, New York, USA).

Statistical differences for mean numbers of CC associated with oocytes from different study groups or developmental potential were calculated by one or two-way ANOVA using the SPSS statistical package (PASW Statistics 18, IBM, New York, USA). In regards to oocyte developmental potential, statistical differences were investigated for $\mathrm{CC}$ numbers for the following developmental stages:

1) COC grade (light or dark);

2) Oocyte maturity (MII or MI+GV, for description see Chapter Methodology);

3) Fertilization rate (2PN; fertilized oocyte) or failed fertilization (FF), including abnormal fertilization);

4) Good embryonic development on Day 3 ( $\geq 7$ cell or $\leq 6$ cell, including FF at fertilization);

5) Blastocyst development (good quality blastocyst used for replacement or frozen for subsequent use and Day 3 embryos that progressed to pregnancy compared to a negative outcome, i.e. embryos that failed to reach blastocyst stage, including those with FF));

6) Pregnancy rate (oocytes that resulted in positive serum hCG level at Day 14 compared with a negative outcome that included unsuitable embryos or those that resulted in negative pregnancy and oocytes with FF) and;

8) Live birth outcome (oocytes that resulted in a live birth compared with a negative outcomes that included unsuitable embryos or those that resulted in negative pregnancy and oocytes with FF) (see Table 4B).

All comparisons were made relative to the total number of MII oocytes available after the denuding step (total N=608; Gp 1, N=270; Gp 2, N=128; Gp 3, N=49; Gp 4, N=161). 


\subsection{Results}

\section{Oocyte developmental indicators}

A total of $608 \mathrm{COC}$ were collected for this study. The respective numbers of COC collected in Gp 1, 2, 3 and 4 were, 270, 128, 49 and 161, respectively.

\section{Morphological characteristics of the Cumulus Cell-Oocyte-Complexes}

The proportion of $\mathrm{COC}$ that were light-shaded compared to those with a dark appearance under the stereo-microscope was highest from women in $\mathrm{Gp} 2$, followed by that from women in Gp 1, and were lowest in COC taken from women in Gp 3 and 4 (see Table 4A).

\section{Oocyte maturity}

Differences in the proportion of MII oocytes reached statistical values only in the comparison of young and healthy (Gp 1) and older and healthy women (Gp 4), where the proportion of MII oocytes in young women was significantly higher $(\mathrm{P}<0.05)$ (see Table $4 \mathrm{~A})$.

\section{Fertilization}

The fertilization rates in Gp 1, Gp 2 and Gp 4 were similar. The fertilization rate in young women with diminished ovarian reserve $(\mathrm{Gp} 3)$ was significantly lower $(\mathrm{P}<0.05)$ compared to other young women (Gp 1 and $\mathrm{Gp} 2$ ) and was similar to older women ( $\mathrm{P}=0.074)$ (see Table4A).

\section{Day 3 embryonic development}

Good embryonic development on Day 3 was similar in embryos from women in Gp 1 and 2, and each was significantly higher compared to that from women in $\mathrm{Gp} 3$ (Gp 1: $\mathrm{P}<0.01, \mathrm{Gp}$ 2: $\mathrm{P}<0.005)$ and $4(\mathrm{Gp} \mathrm{1:} \mathrm{P}<0.05, \mathrm{Gp} 2: \mathrm{P}<0.005)$. The Day 3 embryo cleavage rate was similar in women in Gp 3 and Gp 4 (see Table 4A).

\section{Blastocyst development}

Blastocyst development tended to be higher in Gp 1 women compared to Gp 2 women $(\mathrm{P}=0.051)$ and was significantly higher $(\mathrm{P}=0.015)$ compared to $\mathrm{Gp} 3$ women., There were no differences in blastocyst development in women from $\mathrm{Gp} 2$ and $3(\mathrm{P}=0.176)$. Due to double 
embryo transfers and to low blastocyst formation rate in older women, the developmental potential in single oocytes was not possible (see Table 4A).

\section{Pregnancy rate}

All women in Gp 1 and 2 underwent single embryo transfer (SET), whilst 9 women in Gp 3 underwent SET and one woman underwent a double embryo transfer (DET). Oocytes retrieved from two women in Gp 3 failed to undergo normal fertilization and thus no embryo was transferred. The majority of older ( $\mathrm{Gp} \mathrm{4})$ women underwent DET. The pregnancy rate as determined by circulating hCG levels and presence of a foetal heart beat (FHB) was significantly higher in young and healthy women (Gp 1) compared to that in women with PCOS (Gp2: hCG, P<0.05; FHB, P<0.01), and diminished ovarian reserve (Gp 3: hCG, $\mathrm{P}=0.001$; FHB, $\mathrm{P}<0.0001$ ) and those of older women (Gp 4: hCG, $\mathrm{P}=0.001$; FHB, $\mathrm{P}<0.005$ ). The pregnancy rate in women in Gp 2, 3 and 4 were similar (see Table 4A).

\section{Live birth outcome}

Following embryo transfer, 39.7\% (27/68) of treatments (oocyte collections) resulted in at least one healthy live born child. All but one young women had singleton deliveries (one women in Gp 1 had identical twins), whilst from the four older women, three had singletons and one had non identical twins. The rate of successful IVF treatments measured by the delivery of a healthy live born child was significantly higher in young and healthy women (Gp 1) compared to young women with PCOS background (Gp 2; $\mathrm{P}<0.01$ ), young women with diminished ovarian reserve $(\mathrm{Gp} \mathrm{3}$; $\mathrm{P}<0.0001)$ and older and healthy women $(\mathrm{Gp} 4$; $\mathrm{P}<0.0001$ ). The live birth rates in Gp 2, Gp 3 and Gp 4 were similar (see Table 4A). 
Table 4A: Summary of the indicators of oocyte development in the four study groups. Different letters ( $a, b$ and $c$ ) not shared by variables across the rows denote significant differences between Gps 1-4 (COC, $\mathrm{P}<0.005$; Genomic maturity, $\mathrm{P}<0.05$; Fertilization, $\mathrm{P}<0.05$; Early development, $\mathrm{P}<0.05$; Blastocyst development, $\mathrm{P}<0.05$; hCG, $\mathrm{P}<0.05$; FHB, $\mathrm{P}<0.01$; Live birth, $\mathrm{P}<0.01$. Indicators for COC, oocyte maturity and embryo development are relative to total COC collected. Indicators for pregnancy outcome are relative to the number of women (follicle aspirations). Abbreviations: n/a = not applicable, as number of blastocysts for Gp 4 could not be calculated due to double emvryo transfers.

\begin{tabular}{|c|c|c|c|c|c|}
\hline Indicators & Unit & Gp 1 & Gp 2 & Gp 3 & Gp 4 \\
\hline \multicolumn{6}{|l|}{ Oocyte (Day 0) } \\
\hline COC (light) & $\begin{array}{c}\% \\
(\#)\end{array}$ & $\begin{array}{l}83.3^{\mathbf{b}} \\
(225 / 270)\end{array}$ & $\begin{array}{c}93.8^{\mathbf{a}} \\
(120 / 128)\end{array}$ & $\begin{array}{l}61.2^{\mathbf{c}} \\
(30 / 49)\end{array}$ & $\begin{array}{c}70.8^{\mathbf{c}} \\
(114 / 161)\end{array}$ \\
\hline $\begin{array}{l}\text { Genomic maturity } \\
\text { (MII) }\end{array}$ & $\begin{array}{c}\% \\
(\#)\end{array}$ & $\begin{array}{c}85.9^{\mathbf{a}} \\
(232 / 270)\end{array}$ & $\begin{array}{l}84.4^{\mathbf{a b}} \\
(108 / 128)\end{array}$ & $\begin{array}{c}77.6^{\mathbf{a b}} \\
(38 / 49)\end{array}$ & $\begin{array}{c}77.6^{\mathbf{b}} \\
(125 / 161)\end{array}$ \\
\hline \multicolumn{6}{|l|}{ Embryo (Day $1-6$ ) } \\
\hline Fertilization $(2 \mathrm{PN})$ & $\begin{array}{c}\% \\
(\#)\end{array}$ & $\begin{array}{c}80.6^{\mathbf{a}} \\
(187 / 232)\end{array}$ & $\begin{array}{l}80.6^{\mathbf{a}} \\
(87 / 108)\end{array}$ & $\begin{array}{l}63.2^{\mathbf{b}} \\
(24 / 38)\end{array}$ & $\begin{array}{l}76.8^{\mathbf{a b}} \\
(96 / 125)\end{array}$ \\
\hline $\begin{array}{l}\text { Early stage } \\
(\geq 7 \text { cell })\end{array}$ & $\begin{array}{c}\% \\
(\#)\end{array}$ & $\begin{array}{c}60.3^{\mathbf{a}} \\
(140 / 232)\end{array}$ & $\begin{array}{l}68.5^{\mathbf{a}} \\
(74 / 108)\end{array}$ & $\begin{array}{l}36.8^{\mathbf{b}} \\
(14 / 38)\end{array}$ & $\begin{array}{l}48.8^{\mathbf{b}} \\
(61 / 125)\end{array}$ \\
\hline Blastocyst & $\begin{array}{c}\% \\
(\#)\end{array}$ & $\begin{array}{c}24.1^{\mathbf{a}} \\
(56 / 232)\end{array}$ & $\begin{array}{l}15.7^{\mathbf{a b}} \\
(17 / 108)\end{array}$ & $\begin{array}{l}7.9^{\mathbf{b}} \\
(3 / 38)\end{array}$ & $\mathrm{n} / \mathrm{a}$ \\
\hline \multicolumn{6}{|l|}{ Pregnancy } \\
\hline hCG (Day 14) & $\begin{array}{c}\% \\
(\#)\end{array}$ & $\begin{array}{l}84.0^{\mathbf{a}} \\
(21 / 25)\end{array}$ & $\begin{array}{l}45.5^{\mathbf{b}} \\
(5 / 11)\end{array}$ & $\begin{array}{c}25.0 \mathbf{b} \\
(3 / 12)\end{array}$ & $\begin{array}{l}35.0^{\mathbf{b}} \\
(7 / 20)\end{array}$ \\
\hline FHB (Week 8) & $\begin{array}{c}\% \\
(\#)\end{array}$ & $\begin{array}{l}76.0^{\mathbf{a}} \\
(19 / 25)\end{array}$ & $\begin{array}{l}27.3^{\mathbf{b}} \\
(3 / 11)\end{array}$ & $\begin{array}{l}8.3^{\mathbf{b}} \\
(1 / 12)\end{array}$ & $\begin{array}{c}30.0 \\
(6 / 20)\end{array}$ \\
\hline Live birth & $\begin{array}{c}\% \\
(\#)\end{array}$ & $\begin{array}{l}76.0^{\mathbf{a}} \\
(19 / 25)\end{array}$ & $\begin{array}{l}27.3^{\mathbf{b}} \\
(3 / 11)\end{array}$ & $\begin{array}{l}8.3^{\mathbf{b}} \\
(1 / 12)\end{array}$ & $\begin{array}{l}20.0^{\mathbf{b}} \\
(4 / 20)\end{array}$ \\
\hline
\end{tabular}




\section{Relationships between CC numbers and oocyte developmental indicators}

Of the 608 CC samples collected, 603 (99.2\%) had detectable levels of RPL19 mRNA (Gp 1: 266/270, Gp 2: 127/128, Gp 3: 49/49, Gp 4: 161/161). The mean \pm SEM number of CC per $\mathrm{CC}$ mass calculated (using the regression equation of log $\mathrm{CC}$ number $=-0.2392 *(R P L 19 \mathrm{Ct}$ value) +8.7523 (derived from Figure 3) from RPL19 mRNA values was $28872 \pm 808$ (Gp 1: $28817 \pm 1279$, Gp 2: $32675 \pm 868$, Gp 3: $26834 \pm 2545$, Gp 4: $26584 \pm 1548)$. From the 603 $\mathrm{CC}$ masses analyised in this study, $503(83.4 \%)$ had a $\mathrm{C}_{\mathrm{T}}$ value for RPL19 that was within the range of the regression equation established based on $\mathrm{CC}$ numbers counted $(\mathrm{N}=20$, Chapter 3). The minimum number of $\mathrm{CC}$ required for a detectable $R P L 19$ level $\left(\mathrm{C}_{\mathrm{T}}=28.99\right)$ was estimated to be 66. For the estimation of the minimum number of $\mathrm{CC}$ required for the measurement of candidate genes using the housekeeping gene RPL19 in the multiplex TaqMan method, a $\mathrm{C}_{\mathrm{T}}$ value of 28.99 was chosen; this value is in agreement with previous reports and manufacturers recommendations, where mRNA levels associated with a $\mathrm{C}_{\mathrm{T}}<29$ are considered in the measurable range, and those with a $C_{T}>29$ are regarded as unmeasurable and no different from background fluorescence (Kuo et al. 2011).

Mean number of CC per CC mass was significantly higher in Gp 2 compared with Gp 4 $(\mathrm{P}<0.01)$, but not higher compared to $\mathrm{Gp} 1(\mathrm{P}=0.076)$ or $\mathrm{Gp} 3(\mathrm{P}=0.058)$. No differences were noted between Gp 1, Gp 3 and Gp 4 (Table 4B).

\section{CC numbers related to COC morphology}

COC graded as light had fewer (Gp 1, Gp 2, Gp 4: $\mathrm{P}<0.0001, \mathrm{Gp} 3: \mathrm{P}=0.001)$ CC compared to those that were graded as dark (Table 4B).

\section{CC numbers related to oocyte maturity}

COC associated with mature (i.e. MII stage) oocytes had fewer $(\mathrm{P}<0.01) \mathrm{CC}$ compared to those associated with an immature (GV and MI stage) oocyte from women in Gp 1 and 4, whilst there was no significant difference in the same analysis between women in Gp 2 and 3 (Table 4B; data from abnormal oocytes were excluded).

\section{CC numbers related to day 1-6 embryological development}

There were no significant differences in $\mathrm{CC}$ numbers of $\mathrm{COC}$ with respect to fertilization success or Day 3 of embryo development in the four groups (Table 4B). Similarly, there were 
no differences in CC numbers in relation to blastocyst outcome in Gp 1, 2 and 3 (Table 4B). Due to DET and to low blastocyst formation rate in older women, single oocyte developmental potential in relation to the above criteria was not possible.

\section{CC numbers related to pregnancy and live birth outcome}

Comparisons for pregnancy and live birth outcomes were only carried out in Gp 1 women and there was no significant effect of $\mathrm{CC}$ number on positive outcome (Table 4B). It was not possible to use data from Gp 2 and 3 women due to low numbers of patients. As DET procedures were used in Gp 4 women, results associated with specific embryos that resulted in pregnancy and blastocyst outcomes were not possible.

Table 4B: Summary of the relationship between CC numbers and oocyte developmental indicators in the four study groups. Different letters ( $a, b$ and $c)$ not shared by variables across the rows denote significant differences between $\mathrm{CC}$ numbers (mean $\pm \mathrm{SEM}$ ) of individual COC between Gps 1-4 and; between CC numbers (mean \pm SEM) of oocytes associated with positive and negative outcomes in each study group in relation to oocyte developmental indicators (\# CC Gps 1-4, P<0.01; Day 0: COC, $\mathrm{P}=0.001$; Oocyte genomic maturity, $\mathrm{P}<0.01$; Day 1-6: Fertilization, not significant (NS), Day 3 embryonic development, NS, Blastocyst development, NS; Pregnancy, NS, Live birth outcome, NS; all indicators are relative to the total number of MII oocytes collected, with measurable RPL19 levels in associated CC). Abbreviations: $\mathrm{NO}=$ negative outcome that included unsuitable embryos or those that resulted in failed pregnancies or oocytes that failed to fertilize , $\mathrm{n} / \mathrm{a}=$ not applicable due to low numbers of women in Gp 2 and 3 and due to DET procedures in Gp 4. 


\section{Indicators}

Gp 1

Gp 2

Gp 3

Gp 4

\begin{tabular}{|c|c|c|c|c|c|c|c|c|}
\hline $\begin{array}{c}\text { \# CC } \\
{[\mathrm{mean} \pm \mathrm{SEM}]}\end{array}$ & \multicolumn{2}{|c|}{$28817 \pm 1280^{a b}$} & \multicolumn{2}{|c|}{$32675 \pm 869^{a}$} & \multicolumn{2}{|c|}{$26834 \pm 2546^{a b}$} & \multicolumn{2}{|c|}{$26585 \pm 1548^{b}$} \\
\hline \multicolumn{9}{|l|}{ Day 0} \\
\hline $\mathrm{COC}$ & Light & Dark & Light & Dark & Light & Dark & Light & Dark \\
\hline $\begin{array}{l}\# \mathrm{CC} \\
{[\mathrm{mean} \pm \mathrm{SEM}]}\end{array}$ & $\begin{array}{c}25273^{b} \\
\pm \\
1013\end{array}$ & $\begin{array}{c}46700^{a} \\
\pm \\
5049\end{array}$ & $\begin{array}{c}30885^{b} \\
\pm \\
1516\end{array}$ & $\begin{array}{c}59309^{\mathbf{a}} \\
\stackrel{ \pm}{8305}\end{array}$ & $\begin{array}{c}20493^{b} \\
\pm \\
1891\end{array}$ & $\begin{array}{c}36847^{\mathbf{a}} \\
\pm \\
5136\end{array}$ & $\begin{array}{c}22206^{b} \\
\pm \\
1434\end{array}$ & $\begin{array}{c}37206^{a} \\
\pm \\
3584\end{array}$ \\
\hline $\begin{array}{l}\text { Oocyte } \\
\text { maturity }\end{array}$ & MII & $\mathrm{GV}+\mathrm{MI}$ & MII & $\mathrm{GV}+\mathrm{MI}$ & MII & $\mathrm{GV}+\mathrm{MI}$ & MII & $\mathrm{GV}+\mathrm{MI}$ \\
\hline $\begin{array}{l}\text { \# CC } \\
{[\text { mean } \pm \text { SEM }]}\end{array}$ & $\begin{array}{c}27614^{b} \\
\pm \\
1156\end{array}$ & $\begin{array}{l}38500^{a} \\
\pm \\
6520\end{array}$ & $\begin{array}{c}32242 \\
\pm \\
1723\end{array}$ & $\begin{array}{c}32321 \\
\pm \\
4752\end{array}$ & $\begin{array}{c}27694 \\
\pm \\
3124\end{array}$ & $\begin{array}{c}24726 \\
\pm \\
5646\end{array}$ & $\begin{array}{c}25293^{b} \\
\pm \\
1559\end{array}$ & $\begin{array}{c}36992^{a} \\
\stackrel{ \pm}{5178}\end{array}$ \\
\hline \multicolumn{9}{|l|}{ Day 1-6 } \\
\hline Fertilization & $2 \mathrm{PN}$ & $\mathrm{FF}$ & $2 \mathrm{PN}$ & $\mathrm{FF}$ & $2 \mathrm{PN}$ & $\mathrm{FF}$ & $2 \mathrm{PN}$ & $\mathrm{FF}$ \\
\hline $\begin{array}{l}\text { \# CC } \\
{[\text { mean } \pm \text { SEM] }}\end{array}$ & $\begin{array}{c}28429 \\
\pm \\
1339\end{array}$ & $\begin{array}{c}24209 \\
\pm \\
2084\end{array}$ & $\begin{array}{c}32393 \\
\pm \\
1888\end{array}$ & $\begin{array}{c}31625 \\
\pm \\
4259\end{array}$ & $\begin{array}{c}25680 \\
\pm \\
3593\end{array}$ & $\begin{array}{c}31147 \\
\pm \\
5898\end{array}$ & $\begin{array}{c}25631 \\
\pm \\
1886\end{array}$ & $\begin{array}{c}24175 \\
\pm \\
2525\end{array}$ \\
\hline Day 3 cleavage & $\geq 7$ cell & $\leq 6$ cell & $\geq 7$ cell & $\leq 6$ cell & $\geq 7$ cell & $\leq 6$ cell & $\geq 7$ cell & $\leq 6$ cell \\
\hline $\begin{array}{l}\text { \# CC } \\
{[\text { mean } \pm \text { SEM] }}\end{array}$ & $\begin{array}{c}28387 \\
\pm \\
1573\end{array}$ & $\begin{array}{c}26452 \\
\pm \\
1671\end{array}$ & $\begin{array}{c}31886 \\
\pm \\
1948\end{array}$ & $\begin{array}{c}33006 \\
\pm \\
3496\end{array}$ & $\begin{array}{c}23912 \\
\pm \\
3785\end{array}$ & $\begin{array}{c}29901 \\
\pm \\
4422\end{array}$ & $\begin{array}{c}22505 \\
\pm \\
1925\end{array}$ & $\begin{array}{c}27951 \\
\pm \\
2397\end{array}$ \\
\hline Blastocyst & Good & NO & Good & NO & Good & NO & Good & NO \\
\hline $\begin{array}{l}\# \mathrm{CC} \\
{[\mathrm{mean} \pm \mathrm{SEM}]}\end{array}$ & $\begin{array}{c}29519 \\
\pm \\
2070\end{array}$ & $\begin{array}{c}27038 \\
\pm \\
1370\end{array}$ & $\begin{array}{c}28719 \\
\pm \\
3769\end{array}$ & $\begin{array}{c}32908 \\
\pm \\
1921\end{array}$ & $\begin{array}{c}22847 \\
\pm \\
4766\end{array}$ & $\begin{array}{c}28110 \\
\pm \\
3369\end{array}$ & $\mathrm{n} / \mathrm{a}$ & $\mathrm{n} / \mathrm{a}$ \\
\hline \multicolumn{9}{|l|}{ Pregnancy } \\
\hline hCG (Day 14) & $\geq 18 \mathrm{iu} / 1$ & NO & $\geq 18 \mathrm{iu} / 1$ & $\mathrm{NO}$ & $\geq 18 \mathrm{iu} / 1$ & NO & $\geq 18 \mathrm{iu} / 1$ & NO \\
\hline $\begin{array}{l}\# \mathrm{CC} \\
{[\mathrm{mean} \pm \mathrm{SEM}]}\end{array}$ & $\begin{array}{c}31857 \\
\pm \\
3636\end{array}$ & $\begin{array}{c}27038 \\
\pm \\
1370\end{array}$ & $\mathrm{n} / \mathrm{a}$ & $\mathrm{n} / \mathrm{a}$ & $\mathrm{n} / \mathrm{a}$ & $\mathrm{n} / \mathrm{a}$ & $\mathrm{n} / \mathrm{a}$ & $\mathrm{n} / \mathrm{a}$ \\
\hline Live birth & LB & NO & LB & $\mathrm{NO}$ & LB & NO & LB & NO \\
\hline $\begin{array}{l}\# \mathrm{CC} \\
{[\mathrm{mean} \pm \mathrm{SEM}]}\end{array}$ & $\begin{array}{c}30861 \\
\pm \\
3951\end{array}$ & $\begin{array}{c}27038 \\
\pm \\
1370\end{array}$ & $\mathrm{n} / \mathrm{a}$ & $\mathrm{n} / \mathrm{a}$ & $\mathrm{n} / \mathrm{a}$ & $\mathrm{n} / \mathrm{a}$ & $\mathrm{n} / \mathrm{a}$ & $\mathrm{n} / \mathrm{a}$ \\
\hline
\end{tabular}




\subsection{Discussion}

The major findings of this study were: 1) that as few as $\sim 70 \mathrm{CC}$ are needed to accurately measure the expression levels of multiple genes by multiplex QPCR using RPL19 as a housekeeping gene and; 2) that the mean \pm SEM number of CC present in individual COC recovered for IVF treatment was $28872 \pm 808$. Previously, in a much smaller study, the mean $\mathrm{CC}$ number in a single $\mathrm{COC}$ was reported to be 11500 (Feuerstein et al. 2007). The differences in $\mathrm{CC}$ numbers reported in the present study compared to that by Feuerstein et al. (2007) are likely to be due to 1) the significantly higher number of individual CC masses analysed in the present study, and/or 2) the differences in the follicular aspiration/oocyte denudation protocols (i.e. pressure of the aspiration pump), that could potentially have detrimental effects on the integrity of the outer layers of the CC mass, and therefore result in a higher cell loss (Larsen et al. 1986).

In regards to the minimal number $(\sim 70)$ of CC needed for reliable QPCR measurements, it has to be noted, that these CC numbers have been validated for RPL19 and genes with similar abundance in CC. It is possible, that for the measurement of more lowly expressed genes, a higher number of CC would be needed.

After morphological analyses of individual COC, it became evident that the shade grading (light to dark) of CC masses is related to cell number. Interestingly, CC masses that contained fewer cells were associated with mature oocytes in healthy patients. However, the relationship between cell number/CC mass and oocyte maturation may be associated with the breakdown of gap junctions in COC containing mature oocytes and thus fewer CC are still associated with the oocyte at collection: this breakdown of gap junctions occurs at the time of CC expansion (Gilula et al. 1978; Larsen et al. 1986). Therefore, the mechanical action of removing $\mathrm{COC}$ from those follicles containing a mature oocyte may result in $\mathrm{CC}$ loss. The finding that individual COC collected from women with PCOS tended to have a higher number of $\mathrm{CC}$ indicates a likely functional difference in $\mathrm{CC}$ of these women after the ovulation trigger. Previous studies have reported that morphologically indistinguishable MII oocytes from PCOS and healthy women exhibit different gene expression profiles (Wood et al. 2007) and our finding perhaps adds further evidence to the hypothesis that communication between the oocyte and the follicular environment is impaired or altered in women with PCOS (Willis et al. 1998; Rice et al. 2005). Nevertheless, the numbers of CC per CC mass 
were no different for those that were associated with fertilization, good embryo development and blastocyst and pregnancy outcome compared to those that were associated with negative outcomes in the four groups. These findings add further evidence to the hypothesis, that morphological characteristics of CC are poor markers for oocyte quality.

The pregnancy and live birth rates achieved by the SET procedures in young women, and the DET procedures in older women in this study were more than comparable with current international standards (Esinler et al. 2005; Klipstein et al. 2005; Saldeen et al. 2007; Sahu et al. 2008; Zander-Fox et al. 2011). Oocyte quality as measured by blastocyst development and pregnancy outcome (live birth) was highest in young and healthy women, and was significantly different to values in women with PCOS, diminished ovarian reserve or older women. These embryological results support the notion that when searching for molecular markers in CC for oocyte developmental potential, it becomes important to account for both age and reproductive health. In this context, the women recruited into Group 1 (i.e. healthy young women) of this study are a key subpopulation for identifying molecular markers to determine oocyte health based on blastocyst and pregnancy outcomes. 


\section{Chapter 5 EFFECT OF AGE, INFERTILITY BACKGROUND AND FSH TREATMENT ON HAS2, FSHR, SLC2A4 (GLUT4) mRNA LEVELS IN CUMULUS CELLS}

\subsection{Introduction}

There have been reports describing the effects of age, BMI, antral follicle count, etc on the success of IVF treatments in women (Chang, Chiang et al. 1998; Gianaroli, Magli et al. 1999; Saldeen, Kallen et al. 2007; Orvieto, Meltcer et al. 2009). It has been reported that factors such as: 1) age (especially $>37$ years) is highly correlated with an increased incidence of embryonic chromosomal abnormalities (Gianaroli, Magli et al. 1999; Wells and Delhanty 2000) and negatively correlated with pregnancy and live birth rate (Lass, Croucher et al. 1998); 2) high BMI (>32 kg/m2) is positively correlated with cancellation rate, poor responses and the doses of gonadotrophins used per treatment cycle (Orvieto, Meltcer et al. 2009; Pinborg, Gaarslev et al. 2011) and negatively correlated with pregnancy (Gillett, Putt et al. 2006); 3) increased basal serum FSH and decreased antral follicle count is positively correlated with treatment cancellation rates and negatively with pregnancy outcome (Toner, Philput et al. 1991; Chang, Chiang et al. 1998); 4) reduced serum AMH is indicative of a poor response and diminished ovarian reserve (van Rooij, Broekmans et al. 2002) and; 5) the presence of PCOS is indicative of compromised oocyte development (Wood, Dumesic et al. 2007).

Previous studies have revealed that some biological and/or treatment variables can influence the expression levels of certain genes in CC. For example, it was proposed that age may alter gene expression in oocytes (Grondahl, Yding Andersen et al. 2010) and in CC (Adriaenssens, Wathlet et al. 2010). It was further suggested that BMI, serum FSH concentrations, the type of gonadotrophin used for ovarian stimulation and serum oestradiol concentrations at the time of the ovulation trigger, can also alter the expression levels of certain genes in CC (Adriaenssens, Wathlet et al. 2010). Indeed, recent studies have confirmed that increased basal serum FSH levels affect the levels of gene expression in COC and that it might be one of the possible causes behind the reduced fertility potential in young women with diminished ovarian reserve (Greenseid, Jindal et al. 2011; Jindal, Greenseid et al. 2012). 
The aims of this study were to investigate the influence of biological variables such as age, basal serum levels of FSH and AMH, BMI, PCOS and different gonadotrophin treatments (e.g. amount of rFSH and duration) on expression levels of candidate genes in CC isolated from individual COC recovered from women with different infertility backgrounds (see general methodology for details). For this specific study, the following three genes were selected:

hyaluronidase synthase 2 (HAS2) is one of the key components in the extracelular matrix with an important role in cell proliferation (Toole 1997), cell migration (Turley 1992), tissue remodeling and wound healing (Kuroda et al. 2001) that is also expressed at peak levels in CC of pre-ovulatory follicles (Pienimaki et al. 2001). Indeed, the expression of HAS2 is critical for the formation and expansion of the CC mass and previously shown to have a positive correlation with age (Adriaenssens et al. 2010);

follicle stimulating hormone receptor (FSHR) is expressed by granulosa cells (in the ovary) and by Sertoli cells (in the testis) and is required for the correct functioning of both female and male gonads (Griswold et al. 1995). In the ovary FSHR expression levels have been shown to be regulated by oocyte secreted factors which in turn are thought to mediate the actions of FSH on follicular cells (Otsuka et al. 2005) and;

solute carrier 2, member 4 (SLC2A4, also known as GLUT4) is an insulin dependent glucose transporter protein with a role in glucose uptake, a process that is fundamental for any mammalian cell (Kaestner et al. 1990). In the COC, the oocytes have a poor capacity to utilize glucose and rely on $\mathrm{CC}$ to supply metabolic intermediates for energy production (Sutton et al. 2003). Furthermore, previously it has been reported that GLUT4 is one of the glucose transporters expressed in CC and its role may be critical in the correct development of the oocyte (Roberts et al. 2004).

\subsection{Materials and Methods}

In total, 68 women were allocated into four study groups as previously described (see Chapter 2 and 4). Hormonal stimulation, COC collection, embryo culture and transfer and luteal support were all performed as described in the General Methodology (Chapter 2 and 4, Appendix 2.1-2.2, 3.1-3.3). For quantification of target mRNA levels in individual CC 
masses, quadriplex reaction mixes were prepared containing forward and reverse primers and TaqMan probes for the candidate genes (HAS2, FSHR, GLUT4) and a housekeeping gene (RPL19) at optimized concentrations. A detailed description of the QPCR methodology is presented in General Methodology (Chapter 2). Gene expression levels of the candidate genes in each sample are presented as fold-changes as previously described.

\section{Statistical analyses}

Gene expression levels are reported as mean fold change \pm S.E.M, whilst biological and treatment variables are reported as mean \pm S.E.M. Data were analysed by either one-or twoway ANOVA using the SPSS statistical package (PASW Statistics 18, IBM, New York, USA). Mean \pm S.E.M. gene expression levels were calculated for each woman from mRNA levels of individual COC and their relationship with the following variables were calculated: 1) biological variables: age, BMI, ethnicity, basal serum FSH, and where possible AMH, levels and; 2) treatment variables: daily doses of FSH used for ovarian stimulation, length of ovarian stimulation (days of stimulation with FSH), number of $\geq 14 \mathrm{~mm}$ follicles at ovarian follicle aspiration, serum oestradiol levels on the day of ovulation trigger and number of COC collected. All correlations between gene expression levels, biological and treatment variables were undertaken using 2-tailed Pearson's R test. For group comparisons of the proportions of samples with measurable GLUT4 gene expression levels, a Chi-Square test was performed (SPSS statistical package, IBM).

\section{$5.3 \quad$ Results}

\section{Biological variables: comparison of age, BMI, serum FSH and AMH levels between study groups}

The results of these studies are summarized in Table 5A.

Age

Amongst the young women in Gp 1-3, the mean age was significantly higher $(\mathrm{P}<0.05)$ for those in Gp 3 (i.e. those with diminished ovarian reserve) compared to those in Gp 1 (healthy) and Gp 2 (PCOS). There were no age differences between Gp 1 and Gp 2. 
One of the conditions of enrolment for inclusion into Gp 1, Gp 3 and Gp 4 was a BMI of less than $<28 \mathrm{~kg} / \mathrm{m}^{2}$. However, for women with PCOS (Gp 2), a BMI equal to or higher than 28 was accepted, according to FA clinicians' recommendations. However, no statistical differences with respect to BMI were noted between the four study groups.

\section{Serum Anti-Müllerian hormone (AMH) levels}

Serum AMH levels were measured (as requested by FA clinicians) in $48.5 \%$ (33/68) of women enrolled for this study. The respective proportions for Gp 1-4 were 40\% (10/25), $36.4 \%$ (4/11), 91.7\% (11/12) and 40\% (8/20). Serum AMH levels were significantly higher in Gp 2, compared to Gp1, (P=0.001) Gp $3(\mathrm{P}<0.0001)$ and $\mathrm{Gp} 4(\mathrm{P}<0.0001)$ women. Gp 1 women had higher serum AMH levels than that of Gp 3 and 4 women $(\mathrm{P}<0.0001)$, whilst there were no differences between women from Gp 3 and $\mathrm{Gp} 4$.

\section{Basal serum FSH}

Basal serum FSH levels were significantly higher in women from Gp 3 compared to that in Gp $1(\mathrm{P}<0.0001)$, Gp $2(\mathrm{P}<0.0001)$ and Gp $4(\mathrm{P}<0.005)$. Women in Gp 4 had higher serum FSH levels than those in Gp 1 or $2(\mathrm{P}<0.0001)$, whilst there were no differences between $\mathrm{Gp}$ 1 and Gp 2 women.

\section{Treatment variables: amount of rFSH, serum oestradiol levels, follicle number and the number of COC collected}

These results are also summarized in Table 5A.

\section{Exogenous rFSH treatment}

There were no differences in the duration of ovarian stimulation with exogenous rFSH between the four treatment groups (Table 5A). There were also no differences in the amount of exogenous rFSH used each day for ovarian stimulation for women in Gp 3 and 4. However the mean daily dose of $\mathrm{rFSH}$ administered to these groups were significantly higher $(\mathrm{P}<0.0001)$ compared to that administered to $\mathrm{Gp} 1$ and 2. Moreover, the mean daily doses of $\mathrm{rFSH}$ used in Gp 2 women were lower $(\mathrm{P}<0.0001)$ than those used in women from Gp 1. 
Serum oestradiol levels

The mean serum oestradiol levels measured on the day of the ovulation trigger were the highest in women from Gp 1 and 2, and lowest in women from Gp 3 (Gp 2, P=0.004; Gp 1, $\mathrm{P}=0.016$ ) with the levels in Gp 4 women being intermediate.

\section{Numbers of large $(\geq 14 \mathrm{~mm})$ follicles and COC collected}

The mean number of $\geq 14 \mathrm{~mm}$ follicles on the day of the ovulation trigger and the mean number of COC collected following transvaginal follicle aspirations were highest in women from $\mathrm{Gp} 1$ and 2. The women in both these groups had significantly higher numbers of follicles present allowing for more COC to be collected, compared to that in Gp 4 (follicles, $\mathrm{P}<0.0001$, COC, $\mathrm{P}<0.05$ ) and Gp 3 (follicles and COC, $\mathrm{P}<0.0001$ ) women. Women in Gp 4 had significantly higher number of follicles $(\mathrm{P}<0.05)$ and subsequently COC collected $(\mathrm{P}<0.005)$ compared to that in Gp 3 women. 
Table 5A: Summary of the biological and treatment variables and CC gene expression levels in the four study groups.

\begin{tabular}{|c|c|c|c|c|c|}
\hline Variable & Unit & $\begin{array}{c}\text { Gp 1 } \\
{[\text { mean } \pm \text { SEM] }}\end{array}$ & $\begin{array}{c}\text { Gp } 2 \\
{[\text { mean } \pm \text { SEM] }}\end{array}$ & $\begin{array}{c}\text { Gp 3 } \\
{[\text { mean } \pm \text { SEM] }}\end{array}$ & $\begin{array}{c}\text { Gp } 4 \\
{[\text { mean } \pm \text { SEM] }}\end{array}$ \\
\hline \multicolumn{6}{|l|}{ Biological } \\
\hline Age & Year & $32.1 \pm 0.7^{\mathbf{c}}$ & $30.6 \pm 1.0^{\mathbf{c}}$ & $34.4 \pm 0.7^{\mathbf{b}}$ & $41.7 \pm 0.5^{\mathbf{a}}$ \\
\hline BMI & $\mathrm{kg} / \mathrm{m}^{2}$ & $24.3 \pm 0.6$ & $25.5 \pm 1.3$ & $25.7 \pm 1.0$ & $24.7 \pm 0.6$ \\
\hline AMH & $\mathrm{ng} / \mathrm{mL}$ & $2.9 \pm 0.3^{\mathbf{b}}$ & $7.7 \pm 1.8^{\mathbf{a}}$ & $0.4 \pm 0.1^{\mathbf{c}}$ & $0.5 \pm 0.1^{\mathbf{c}}$ \\
\hline Basal FSH & $\mathrm{IU} / \mathrm{L}$ & $5.6 \pm 0.3^{\mathbf{c}}$ & $4.8 \pm 0.5^{\mathbf{c}}$ & $11.2 \pm 0.6^{\mathbf{a}}$ & $8.6 \pm 0.5^{\mathbf{b}}$ \\
\hline \multicolumn{6}{|l|}{ Treatment } \\
\hline rFSH (days) & $\#$ & $11.0 \pm 0.3$ & $11.6 \pm 0.5$ & $11.4 \pm 0.5$ & $11.4 \pm 0.3$ \\
\hline rFSH (daily) & IU & $147 \pm 3.5^{\mathbf{b}}$ & $100 \pm 7.1^{\text {c }}$ & $293 \pm 6.2^{\mathbf{a}}$ & $279 \pm 8^{\mathbf{a}}$ \\
\hline $\mathrm{E}^{2}$ trigger & $\mathrm{pmol} / \mathrm{L}$ & $7343 \pm 622^{a}$ & $8242 \pm 534^{\mathbf{a}}$ & $4529 \pm 967^{\mathbf{b}}$ & $6486 \pm 682^{\mathbf{a b}}$ \\
\hline$\geq 14$ mm follicles & $\#$ & $11.0 \pm 0.5^{\mathbf{a}}$ & $11.6 \pm 1.2^{\mathbf{a}}$ & $4.3 \pm 0.6^{\mathbf{c}}$ & $6.5 \pm 0.7^{\mathbf{b}}$ \\
\hline COC collected & $\#$ & $11.0 \pm 0.8^{\mathbf{a}}$ & $11.6 \pm 1.1^{\mathbf{a}}$ & $4.1 \pm 0.7^{\mathbf{c}}$ & $8.1 \pm 0.8^{\mathbf{b}}$ \\
\hline \multicolumn{6}{|l|}{$\mathrm{CC}$ gene expression } \\
\hline$H A S 2$ & $\begin{array}{l}\text { Arbitrary } \\
\text { value }\end{array}$ & $2.87 \pm 0.15^{\mathbf{b}}$ & $2.18 \pm 0.15^{\mathbf{c}}$ & $3.99 \pm 0.30^{\mathbf{a}}$ & $4.24 \pm 0.22^{\mathbf{a}}$ \\
\hline FSHR & $\begin{array}{l}\text { Arbitrary } \\
\text { value }\end{array}$ & $0.55 \pm 0.02{ }^{\mathbf{c}}$ & $0.70 \pm 0.02^{\mathbf{a}}$ & $0.56 \pm 0.04^{\mathbf{b c}}$ & $0.64 \pm 0.02^{\mathbf{a b}}$ \\
\hline $\begin{array}{l}\text { CC with measurable } \\
\text { GLUT4 }\end{array}$ & $\%$ & $5.19^{\mathbf{b}}$ & $3.91^{\mathbf{b}}$ & $6.12^{\mathbf{a b}}$ & $14.91^{\mathbf{a}}$ \\
\hline
\end{tabular}

Different letters ( $a, b$ and $c$ ) not shared by variables across the rows denote significant differences between Gps 1-4 (age, P<0.05; BMI, not significant (NS); AMH, P<0.001; basal serum FSH, $\mathrm{P}<0.005$; days of $\mathrm{rFSH}$, NS; daily $\mathrm{rFSH}, \mathrm{P}<0.0001$; total $\mathrm{rFSH}, \mathrm{P}=0.001$; $\mathrm{E}^{2}$ trigger, $\mathrm{P}<0.05$; number of $\geq 14 \mathrm{~mm}$ follicles, $\mathrm{P}<0.05$; number of $\mathrm{COC}, \mathrm{P}<0.05$; HAS2 gene expression, $\mathrm{P}<0.005 ;$ FSHR gene expression, $\mathrm{P}<0.05$; \% measurable GLUT4, $\mathrm{P}=0.001$ ). 


\section{Gene expression levels and interrelationships}

Expression levels of HAS2 and FSHR mRNA were detectable in 99.2\% (603/608) of the CC samples. Overall, the relative mean levels of HAS2 were higher $(\mathrm{P}<0.0001)$ than those for FSHR mRNA. Furthermore, there was a significant $(\mathrm{P}<0.0001)$ overall linear correlation between the $\mathrm{C}_{\mathrm{T}}$ values for $H A S 2$ and FSHR in individual CC masses when the data for the

four treatment groups were pooled $\left(\mathrm{R}^{2}=0.6761\right)$. The respective $\mathrm{R}^{2}$ values for the individual groups were also highly correlated (Figure 5). 
1)

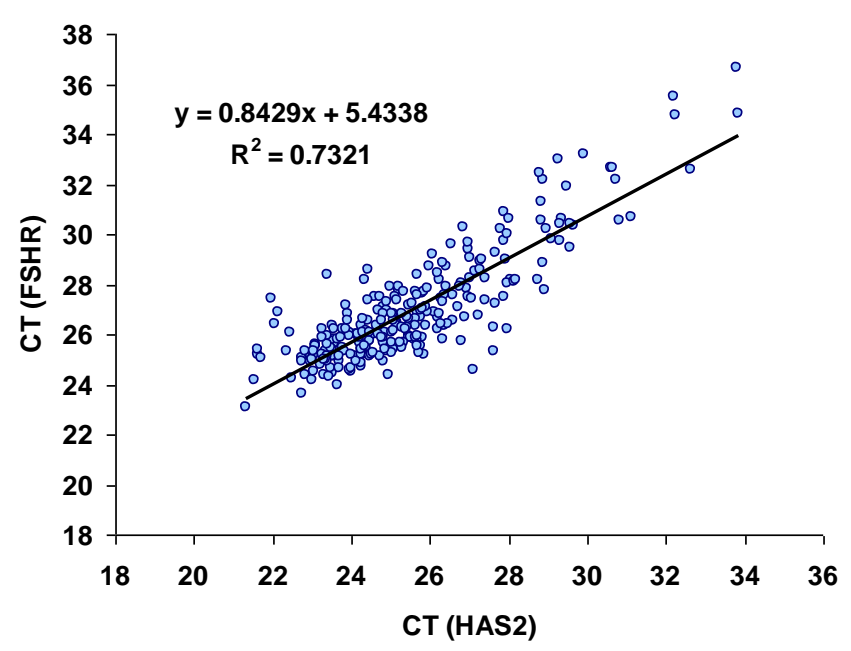

3)

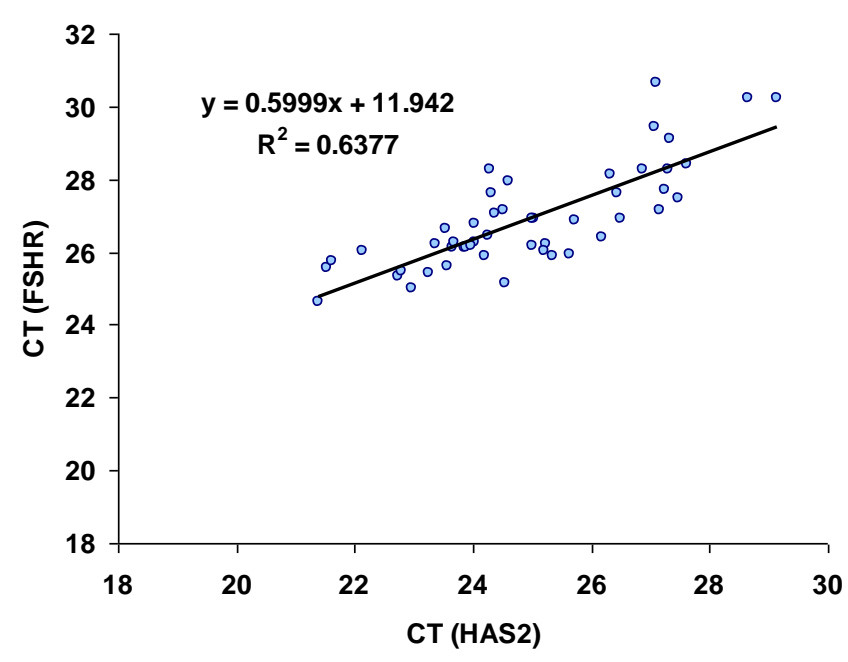

2)

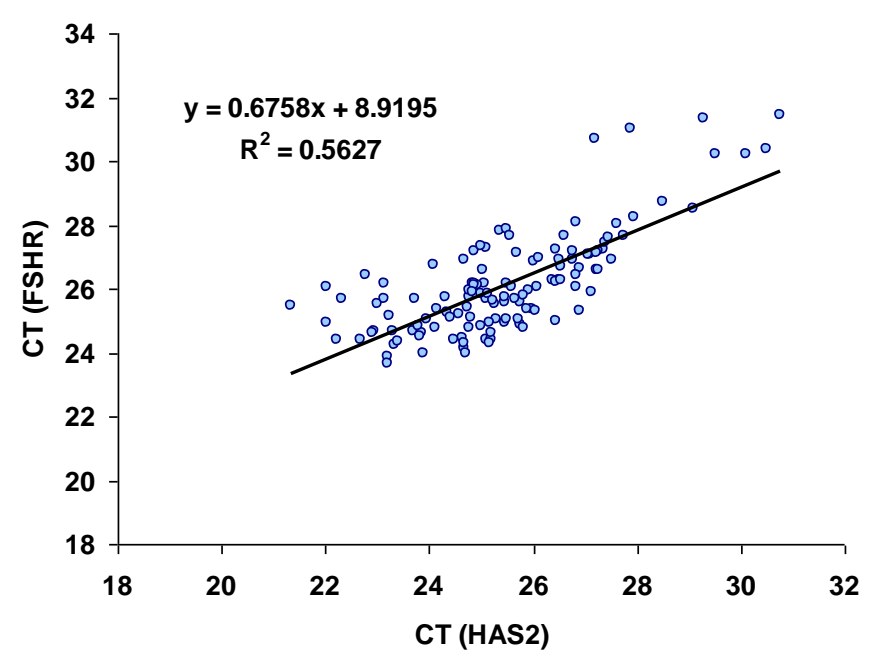

4)

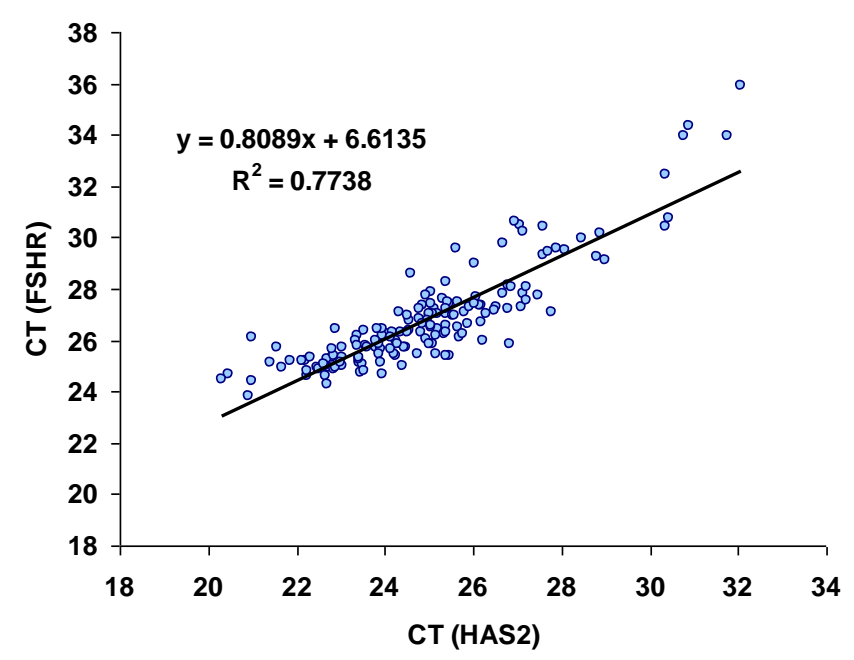

Figure 5: Linear regressions of Cycle Threshold $\left(\mathrm{C}_{\mathrm{T}}\right)$ values of HAS2 and FSHR mRNA in CC masses from women in Gp 1 (1), Gp 2 (2), Gp 3 (3), and Gp 4 (4).

Expression levels of HAS2 mRNA were not different in CC recovered from women in Gp 3 and 4 but both were significantly higher $(\mathrm{P}<0.005)$ compared to that from $\mathrm{Gp} 1$ and 2 . Furthermore, HAS2 expression levels were significantly higher $(\mathrm{P}=0.004)$ in $\mathrm{CC}$ recovered from women in Gp 1 compared to Gp 2 (Table 5A). Expression levels of FSHR were lowest in $\mathrm{CC}$ recovered from women in Gp 1 and 3, and highest in $\mathrm{CC}$ recovered from women in Gp 
2 and 4 (Table 5A). The mRNA expression levels of GLUT4 were detectable in only $7.6 \%$ of samples. The respective proportions in Gps 1-4 were 5.2\% (14/270), 3.9\% (5/128), 6.1\% (3/49) and 14.9\% (24/161). The proportion of measurable GLUT4 levels was highest in Gp4 (Table 5A). Differences in mean GLUT4 gene expression levels between the four groups were not analysed due to the low percentage of samples with detectable expression levels.

\section{Interrelationships amongst $\mathrm{CC}$ gene expression levels and biological and treatment variables}

\section{HAS2 gene expression levels}

There was a highly significant linear correlation between the HAS2 gene expression levels and basal serum FSH levels (Table 5B) and daily doses of rFSH (Table 5B) used for ovarian stimulations. There were highly significant linear correlations of HAS2 mRNA levels with patient age (Table 5B) and with serum AMH levels (Table 5B). Furthermore, there were highly significant linear correlations between age, basal FSH and AMH levels (Appendix 5). However, there was no significant correlation between HAS2 mRNA levels and BMI (Tabel $5 B)$. In regards to other treatment variables, there were highly significant linear correlations between HAS $2 \mathrm{mRNA}$ levels and the numbers of $\geq 14 \mathrm{~mm}$ follicles present on the day of the ovulation trigger, and also the mean number of COC collected following transvaginal follicle aspirations (Table 5B). However, there were no correlations between HAS2 mRNA levels and serum oestradiol levels (Table 5B). The amounts of $\mathrm{rFSH}$ used for ovarian stimulation in individual woman were highly correlated with oestradiol levels $(\mathrm{P}=0.001)$ and the numbers of $\geq 14 \mathrm{~mm}$ follicles $(\mathrm{P}<0.0001)$ measured on the day of ovulation trigger, as well as the number of COC collected $(\mathrm{P}<0.0001)$ following transvaginal follicle aspiration (Appendix 5). There was also a highly significant correlation between the numbers of follicles $\geq 14 \mathrm{~mm}$ and number of COC collected $(\mathrm{P}<0.0001)$, and with oestradiol levels measured on the day of ovulation trigger $(\mathrm{P}<0.0001)$ (Appendix 5). 
Table 5B: Summary showing the Pearson correlation coefficients and significance levels (P values) between HAS2 or FSHR mRNA levels and biological and treatment variables in the 68 women recruited in the four study groups. NS refers to no correlation $(\mathrm{P}>0.05)$.

\begin{tabular}{lcccc}
\hline Variable & \multicolumn{2}{c}{ HAS2 } & \multicolumn{2}{c}{ FSHR } \\
\hline & Pearson's R & Sig. (P value) & Pearson's R & Sig. (P value) \\
\hline Biological & & & & \\
Age & 0.324 & 0.007 & -0.029 & NS \\
BMI & 0.042 & NS & -0.072 & NS \\
AMH & -0.512 & 0.002 & 0.055 & NS \\
Basal FSH & 0.309 & 0.011 & -0.064 & NS \\
Treatment & & & & \\
rFSH (days) & -0.031 & NS & 0.080 & NS \\
rFSH (daily) & 0.453 & $<0.0001$ & 0.034 & NS \\
E ${ }^{2}$ trigger & -0.205 & NS & -0.178 & NS \\
$\geq 14$ mm & -0.375 & 0.002 & -0.016 & NS \\
follicles & -0.321 & 0.008 & 0.009 & NS \\
COC collected & & & &
\end{tabular}

FSHR gene expression levels

There were no significant linear correlations between FSHR mRNA levels in CC and biological or treatment variables (Table 5B).

\section{GLUT4 gene expression levels}

The proportion of samples with measurable GLUT4 expression was not correlated with patient age when the data were analysed for individuals (Pearson's $\mathrm{R}=0.222, \mathrm{P}=0.069$ ) but was significantly correlated when the data for each of the treatment groups were pooled 
(Pearson's $\mathrm{R}=0.987, \mathrm{P}<0.05$ ) (data not shown). With regards to GLUT4 expression and other biological and treatment variables, no other correlations were observed.

\subsection{Discussion}

Our data supports the concept that the ovarian and endocrine status, as well as rFSH treatment, seems to have profound effects on the levels of gene expression in $\mathrm{CC}$ (Adriaenssens et al. 2010). This interpretation is based on an examination of the levels of the two highly expressed genes (HAS2 and FSHR) and the abundance of a lowly-expressed gene (GLUT4) which, to varying degrees differed in individuals recruited in the four study groups.

The mean expression levels of HAS2 mRNA were the highest in women with diminished ovarian reserve (young and older) and the lowest in women with PCOS. On the other hand, expression levels of FSHR mRNA were the highest in young women with PCOS and lowest in young and healthy women. Furthermore, the proportion of CC masses with measurable GLUT4 levels was highest in women $\geq 40 \mathrm{yr}$ of age (Gp 4). Previous studies that have investigated CC and GC gene expression levels in relation to infertility reported that some genes (i.e. the IGF gene family, Gremlin 1) are impaired in young women with diminished ovarian reserve compared with those with a normal age-related reserve, highlighting the importance of ovarian factors (Greenseid et al. 2011; Jindal et al. 2012).

The mean expression levels of FSHR mRNA in individual CC masses were the highest in women with PCOS. This finding correlates well with previous reports, where it was noted that FSHR mRNA levels are higher in GC (pooled from several follicles) from women with PCOS compared with women with normal ovarian function and women with poor ovarian reserve (Almahbobi et al. 1996; Cai et al. 2007; Catteau-Jonard et al. 2008; GonzalezFernandez et al. 2010). However, in these studies, the FSHR mRNA expression levels in pooled GC were lower in women with poor ovarian reserve compared with control whereas in the present study no differences were found for individual CC with the same comparison.

The relationship analyses revealed that the HAS2 mRNA levels showed the strongest association with the exogenous rFSH used for ovarian stimulation. There is substantial evidence to show that HAS2 expression is markedly up-regulated in COC undergoing cumulus expansion and final oocyte maturation under the regulation of gonadotrophins 
(Kimura et al. 2002; Ochsner et al. 2003; Schoenfelder and Einspanier 2003; Su et al. 2003; Romero et al. 2011). Indeed, cumulus expansion requires gonadotrophin-dependent upregulation of several key mucification genes including PTGS2, PTX3, TNFAIP6 as well as HAS2 (Elvin et al. 1999; Fulop et al. 2003; Salustri et al. 2004). Therefore, the strong correlation between the mean HAS2 and FSHR expression levels in CC is not surprising and is consistent with the increased production of hyaluronic acid in COC in response to FSH (Eppig 1979; Salustri et al. 1989; Buccione et al. 1990b). Furthermore, HAS2 gene expression level was positively correlated with basal serum FSH and age, and negatively correlated with serum AMH levels, numbers of large follicles $(\geq 14 \mathrm{~mm})$ on the day of ovulation trigger and also with numbers of oocytes collected. The tight correlations between basal FSH levels with certain biological variables and treatments are consistent with previous reports. For example, previously it has been shown that basal serum FSH levels increase progressively with reproductive age (Ahmed Ebbiary et al. 1994) and the age-related increases in doses of gonadotrophins used for ovarian stimulation in IVF (Sharif et al. 1998). Moreover, basal FSH levels have been reported to be negatively correlated with serum AMH levels (Pigny et al. 2003), peak serum oestradiol levels (Hohmann et al. 2005), numbers of large follicles present on the ovaries and thus oocytes collected (Scott et al. 1989; Ebrahim et al. 1993). In regards to previous gene expression studies, HAS2 mRNA levels in CC have been reported to be positively correlated to age (Adriaenssens et al. 2010), however positive associations of HAS2 mRNA levels to other variables such as basal serum FSH concentrations, doses of rFSH used for stimulation, serum oestradiol levels, numbers of large follicles present and thus to numbers of oocytes collected have not been reported hitherto. The highly significant correlation between HAS2 expression levels, the amount of rFSH used for stimulation and the basal serum FSH concentrations, taking into account the role of FSH in regards to hyaluronic acid synthesis in CC, confirms that HAS2 gene expression levels in $\mathrm{CC}$ is primarily correlated to serum FSH levels. The influence of age may only be a secondary factor, as this correlation could be, at least partly, attributed to increased basal serum FSH levels associated to older women (Ahmed Ebbiary et al. 1994).

With regards to biological and treatment variables, the expression levels of FSHR mRNA in CC showed no relationship to any of the variables.

The finding that the proportion of measurable GLUT4 samples tended to be positively correlated with age is perhaps surprising as the risk of developing insulin resistance increases 
with age (Defronzo 1979): the abundance of GLUT 4 mRNA is tightly correlated with insulin responsiveness in porcine and human granulosa cells (Yan et al. 2009; Rice et al. 2011) and human adipocytes (Rosenbaum et al. 1993). Interestingly, expression levels of GLUT 1 and 3, and to a lesser extent GLUT 4, were similar in bovine follicles at all developmental stages, with the exception of dominant oestrogen-inactive follicles undergoing atresia whereby message levels were significantly higher and inversely proportional to glucose concentrations in follicular fluid (Nishimoto et al. 2006). Therefore, it is possible that a higher proportion of COC obtained from the older women in this study were from follicles beginning to undergo atresia.

In conclusion, the present study provides further evidence that the expression of some candidate genes in CC may be affected by various biological and/or treatment variables. Thus, in searching for gene expression markers in $\mathrm{CC}$ to determine the selection of oocytes for positive pregnancy outcomes these and other possible variables should always be taken in consideration. 


\section{Chapter 6 MESSENGER RNA LEVELS IN CUMULUS CELLS: RELATIONSHIPS TO EMBRYOLOGICAL AND PREGNANCY OUTCOMES}

\subsection{Introduction}

In recent years, several research groups have explored the possibility that gene expression activity in CC may provide a non-invasive method during IVF of selecting oocytes with a greater likelihood of a successful pregnancy outcome. The first genes investigated were genes responsible for the synthesis of the COC extracellular matrix proteins, hyaluronidase synthase 2 (HAS2), pentraxin 3 (PTX3), tumour necrosis factor $\alpha$-induced protein 6 (TNFAIP6) and those involved in cumulus cell signalling such as gremlin 1 (GREMI) and prostaglandinendoperoxide synthase 2 (PTGS2) (McKenzie, Pangas et al. 2004). Several subsequent studies investigated the same group of genes, however the results have proved to be inconclusive and variable (Zhang, Jafari et al. 2005; Cillo, Brevini et al. 2007; Anderson, Sciorio et al. 2009). Currently, many more genes have been investigated, such as those involved in the secretion of COC extracellular matrix (versican), cumulus cell signalling (amphiregulin, connexin 43, aryl hydrocarbon receptor, steroid sulfatase, steroidogenic acute regulatory protein, syndecan 4 , calmodulin 2 , etc), metabolic pathways (stearoyl-CoA desaturase 1 and 5, aldolase A, lactate dehydrogenase A, phosphofructokinase, pyruvate kinase, etc) (Feuerstein, Cadoret et al. 2007; Adriaenssens, Wathlet et al. 2010; Gebhardt, Feil et al. 2011; Wathlet, Adriaenssens et al. 2011). Although some of these studies showed that mRNA levels in $\mathrm{CC}$ have the potential to fulfil the criteria for oocyte quality markers in selected groups of oocytes, none have reported significant associations of gene expression levels to good blastocyst development and pregnancy outcome in relation to total number of oocytes available after follicular aspirations.

The aims of the present study were to investigate the abundance of and interrelationships amongst a selected number of CC expressed genes and to determine whether the mRNA expression levels of these genes were correlated to oocyte quality as measured by blastocyst development and pregnancy outcome after follicular aspirations in young and healthy women.

For these investigations the following six candidate genes were selected: 
hyaluronidase synthase 2 (HAS2) and follicle stimulating hormone receptor (FSHR) (see Introduction part of Chapter 5 for details);

versican $(V C A N)$ is a chondroitin sulfate proteoglycan, a major component of the COC extracellular matrix that binds to hyaluronan (Naso et al. 1994). Versican has major roles in the control of cellular growth, cell differentiation (Naso et al. 1994), cell adhesion, migration and regeneration (Lebaron 1996). Furthermore, it has previously been reported as one of the most promising oocyte quality markers in CC (Adriaenssens et al. 2010; Gebhardt et al. 2011; Wathlet et al. 2011);

activated leukocyte cell adhesion molecule (ALCAM), is a gene that belongs to the immunoglobulin family, which mediates homophilic (ALCAM - ALCAM) and heterophilic (ALCAM-CD6) cell to cell interaction (Bowen and Aruffo 1999). It is known to be involved in cell adhesion and/or signaling (Bowen and Aruffo 1999), cell migration (Sanders et al. 2011), in the formation and function of the lymphatic system (Iolyeva et al. 2012) and in the initial step of human embryo implantation (Fujiwara et al. 2003). More importantly it is expressed in $\mathrm{CC}$ and it has been proposed that could be a potential oocyte quality marker (Adriaenssens et al. 2010; Wathlet et al. 2011);

progesterone receptor $(P R)$, a gene expressed in humans in the dominant follicle following the initiation of the LH surge and continues to be expressed also in the active corpus luteum (Iwai et al. 1990). The expression of $P R$ is critical for follicular growth and development, luteinization and successful ovulation (Robker et al. 2009). It is also essential for a successful implantation although little is known about the role of this gene during the peri-conception period (Robker et al. 2009) and;

neuropilin 1 (NRPl) is a membrane-bound co-receptor to tyrosine kinase receptor for vascular endothelial growth factor and members of the semaphorin family proteins. Neuropilin 1 is involved in the angiogenic and neuronal traffic through the cytoskeleton, in cell assembly and interaction and more importantly in vesicle guidance and transport in follicles following oestradiol induction. Moreover, in a preliminary study it was reported that its up-regulation gene expression in CC may potentially indicate oocyte with positive pregnancy outcome (Assidi et al. 2011). 


\subsection{Materials and Methods}

In total, $270 \mathrm{COC}$ were collected from 25 young and healthy women (Gp 1). Hormonal stimulation, COC collection, embryo culture and transfer and luteal support were all performed as described in the General Methodology (Chapter 2 and 4, Appendix 2.1-2.2 and 3.1-3.3). For quantification of target mRNA levels in individual $\mathrm{CC}$ masses, multiplex reaction mixes were prepared containing forward and reverse primers and TaqMan probes for the candidate genes [HAS2, FSHR, GLUT4 (Reaction 1); SFRP2, ALCAM (Reaction 2); and $V C A N, P R$ and NRP1 (Reaction3)] and the housekeeping gene (RPL19) at optimized concentrations. A detailed description of the QPCR methodology including measurements of relative gene expression levels using the calibrator and RPL19 are presented and/or validated in General Methodology (Chapter 2) and Chapter 3.

\section{Statistical analyses}

Gene expression levels are reported as mean fold change \pm S.E.M. Data were subjected to either one-or two-way ANOVA using the SPSS statistical package (PASW Statistics 18, IBM, New York, USA). The expression levels of candidate genes were assessed in relation to oocyte developmental stages as described in Chapter 4 so that appropriate comparisons with published reports can be made (Adriaenssens et al. 2010; Wathlet et al. 2011). In brief, statistical differences were calculated for candidate mRNA levels in relation to 1) COC morphology, 2) oocyte maturity, 3) fertilization rate, 4) good embryonic development on day 3, 5) blastocyst development, 6) pregnancy rate and, 7) live birth outcome. All comparisons were made relative to the total number $(\mathrm{N}=270)$ of oocytes collected following follicle aspirations.

\subsection{Results}

\section{Gene expression levels in cumulus cells}

Expression levels of HAS2, FSHR, ALCAM, VCAN, NRP1 and PR mRNA were detectable in $98.5 \%(266 / 270)$ of samples, whilst the proportion of samples with detectable levels of GLUT4 and SFRP2 were low (5.2 and 0.4\% respectively). Overall, the relative expression levels of VCAN mRNA were the highest, followed by those of NRP1, HAS2, ALCAM, FSHR 
and $P R$ : the statistical differences were $V C A N$ vs $N R P 1, \mathrm{P}<0.0001 ; N R P 1$ vs $H A S 2$, $\mathrm{P}<0.0001 ; H A S 2$ vs $A L C A M, \mathrm{P}<0.05 ; A L C A M$ vs FSHR, $\mathrm{P}<0.0001$ and; FSHR vs $P R, \mathrm{NS})$. There were highly significant $(\mathrm{P}<0.0001)$ linear correlations in raw $\mathrm{C}_{\mathrm{T}}$ values between all six abundant candidate genes in individual CC masses (Figure 6A). The relationship between these six genes (normalized gene expression $\left(2^{\wedge}(-\Delta \Delta C T)\right.$ values)) was also verified using principal component analysis (PCA) using the software R (www.r-project.org/), where similar results were noted. Analysing the expression data of the six genes derived from the $227 \mathrm{COC}$, it was found that the first two principal components explained $67.7 \%$ of the total variance (Appendix 6). 
1)

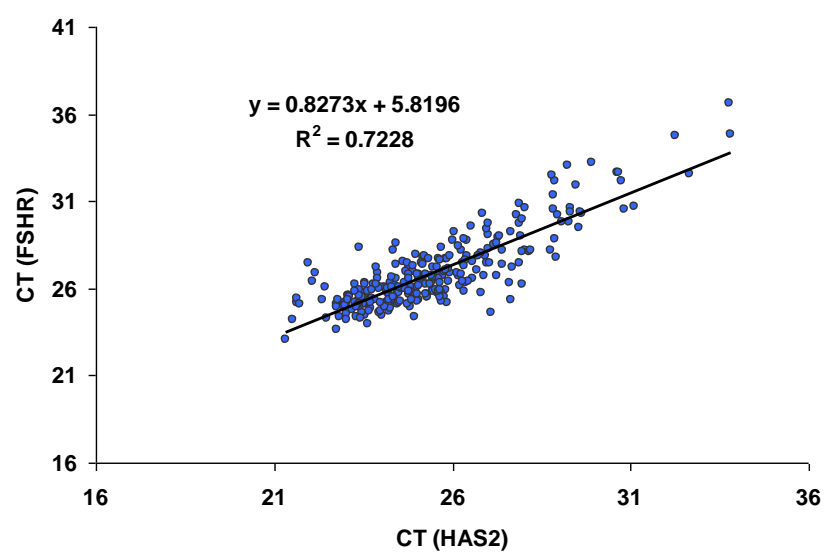

3)

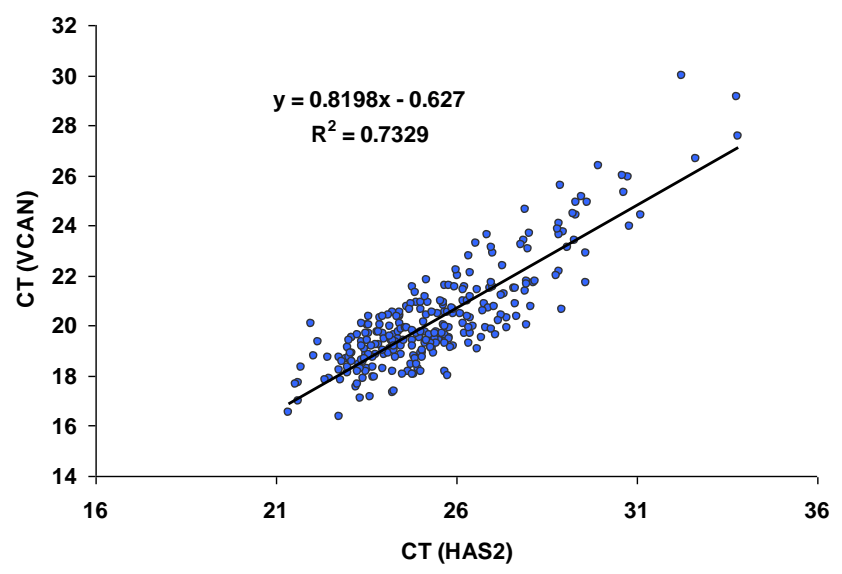

5)

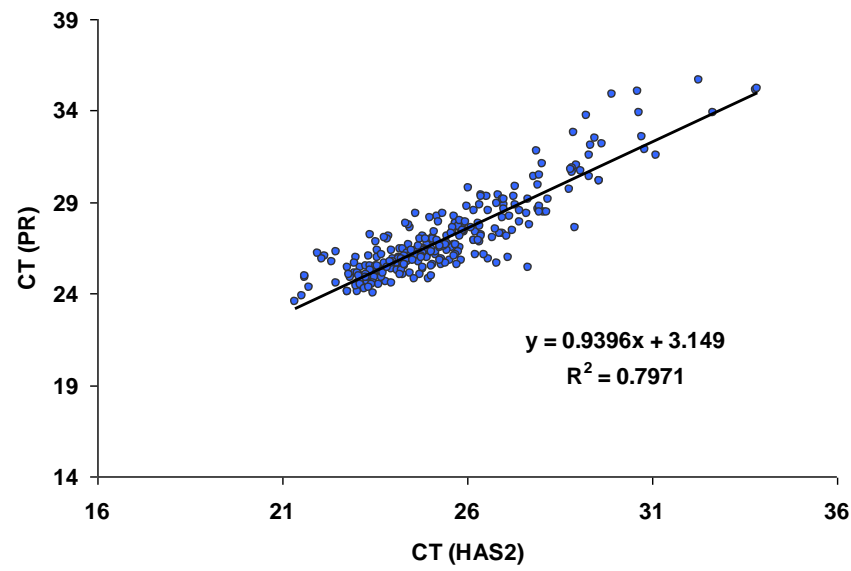

2)

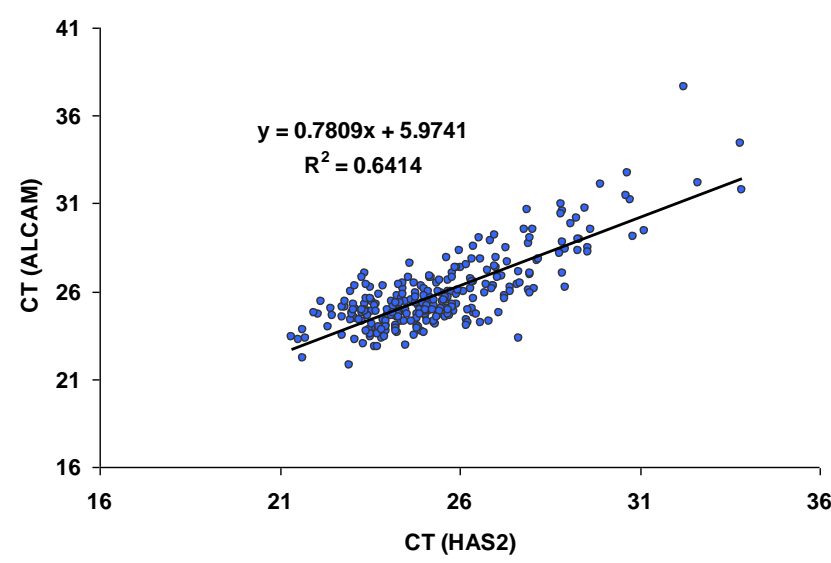

4)

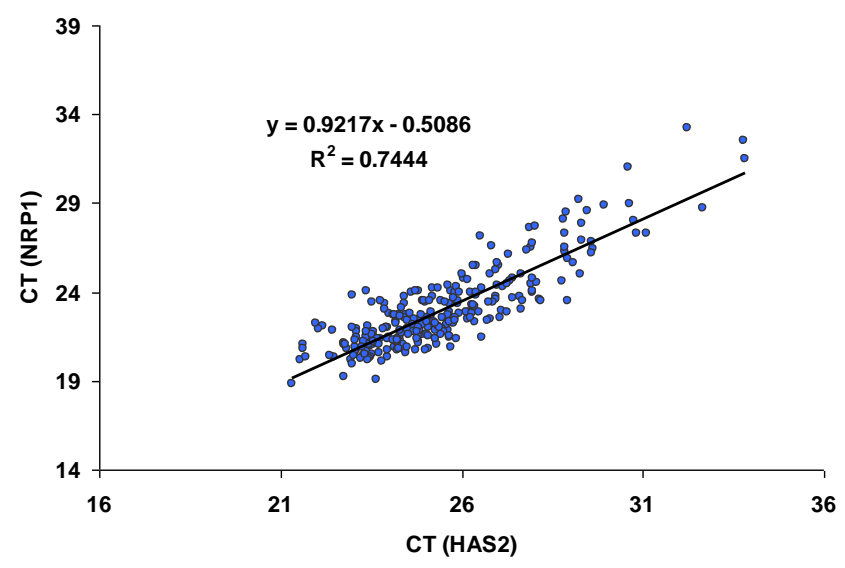

6)

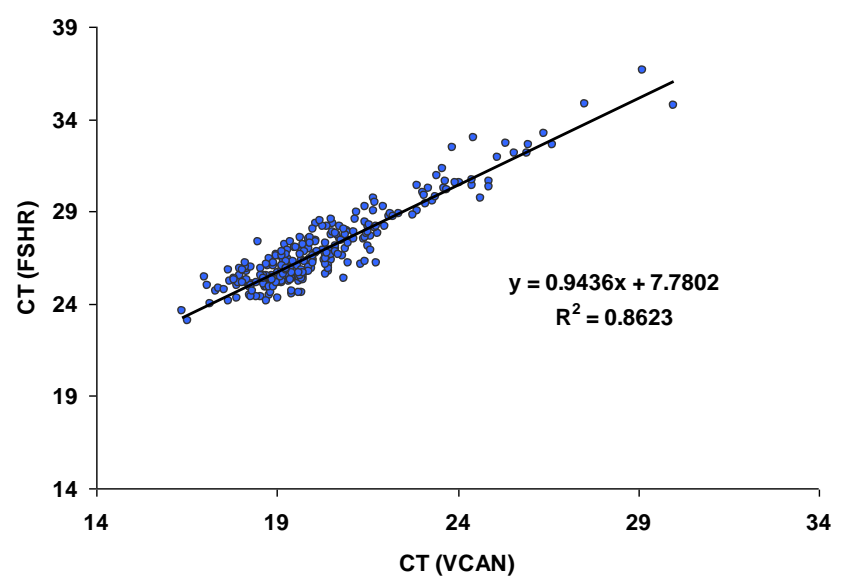


7)

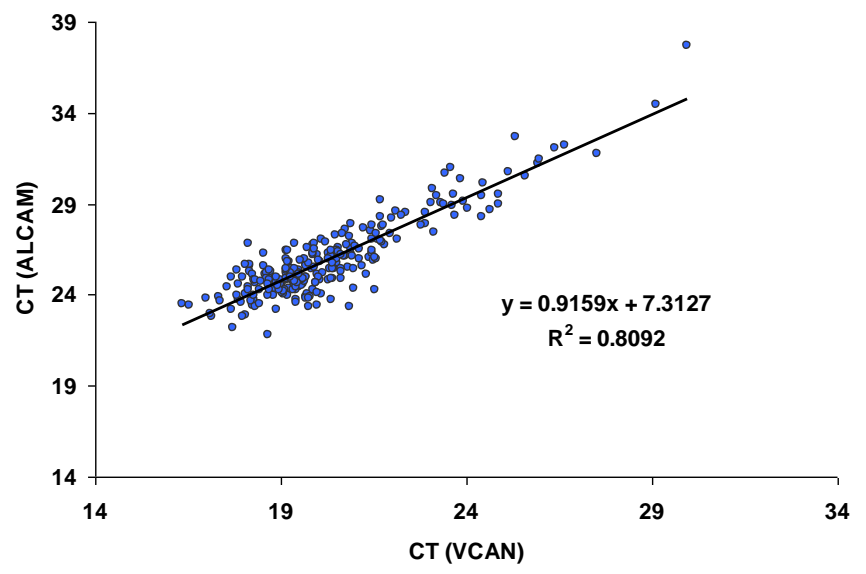

9)

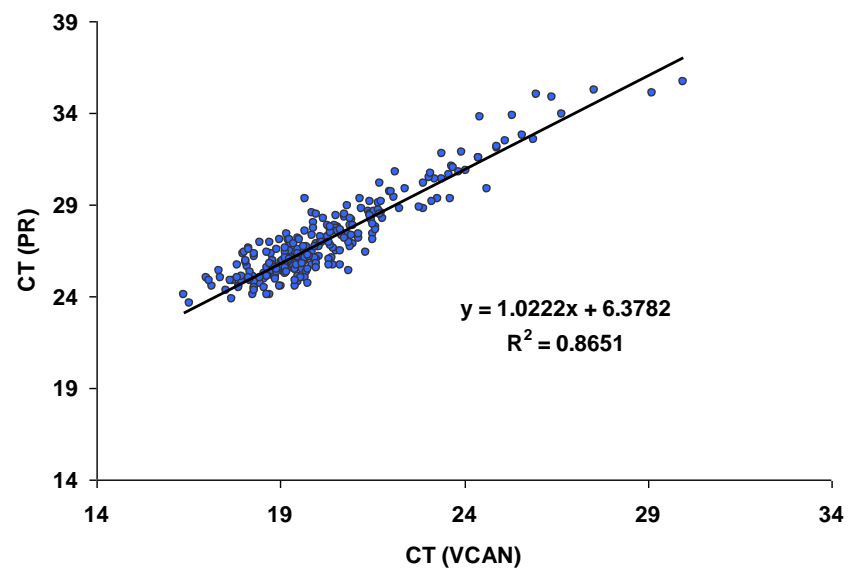

11)

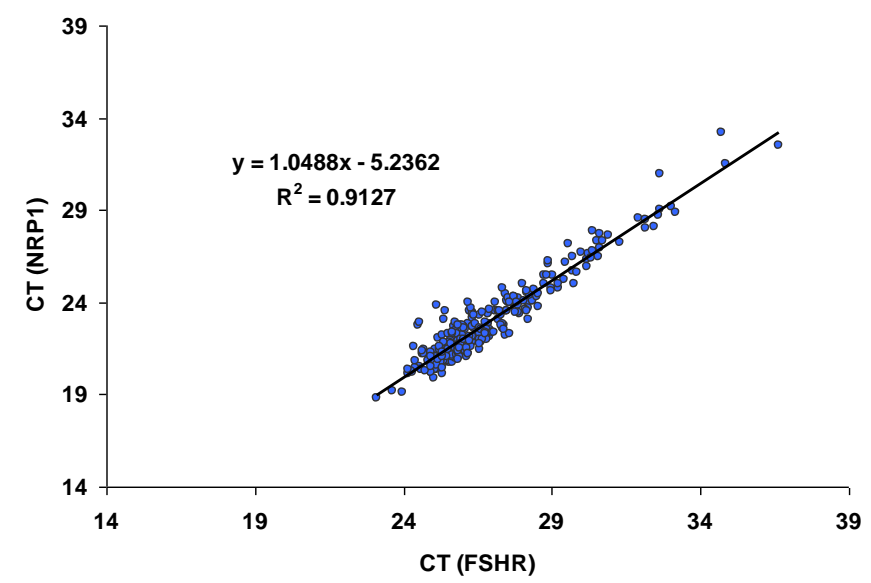

8)

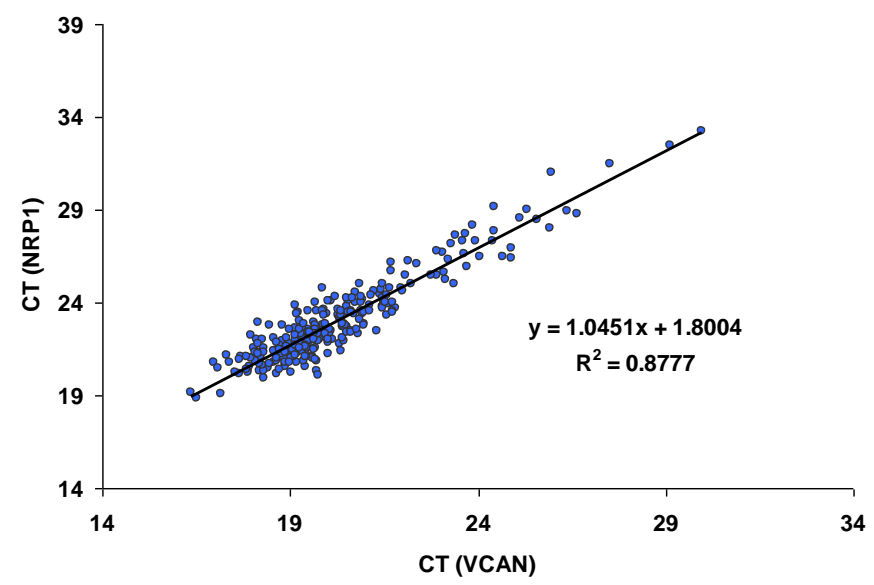

10)

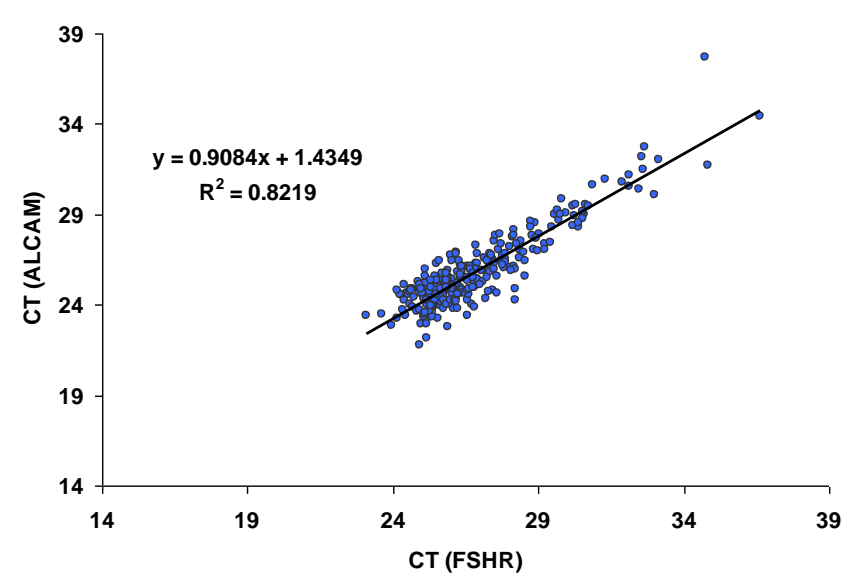

12)

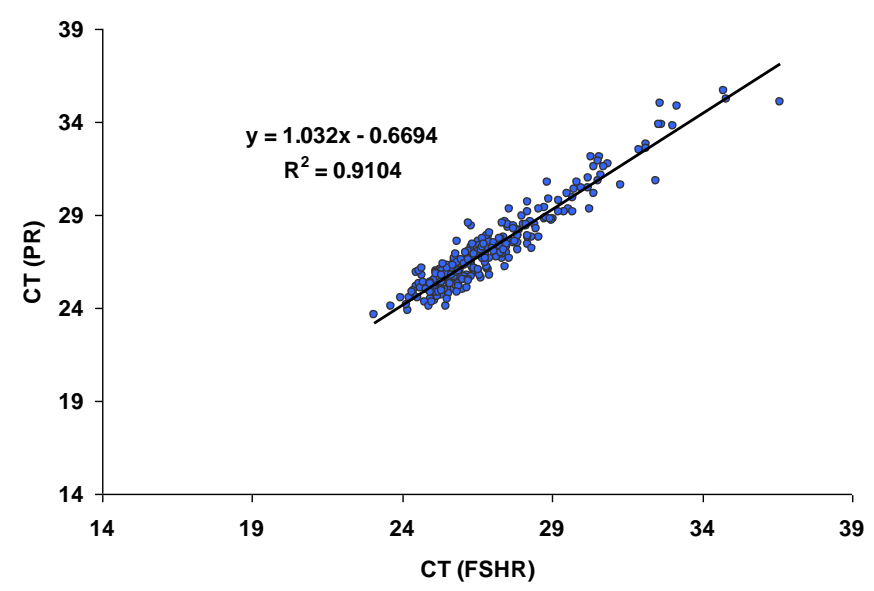



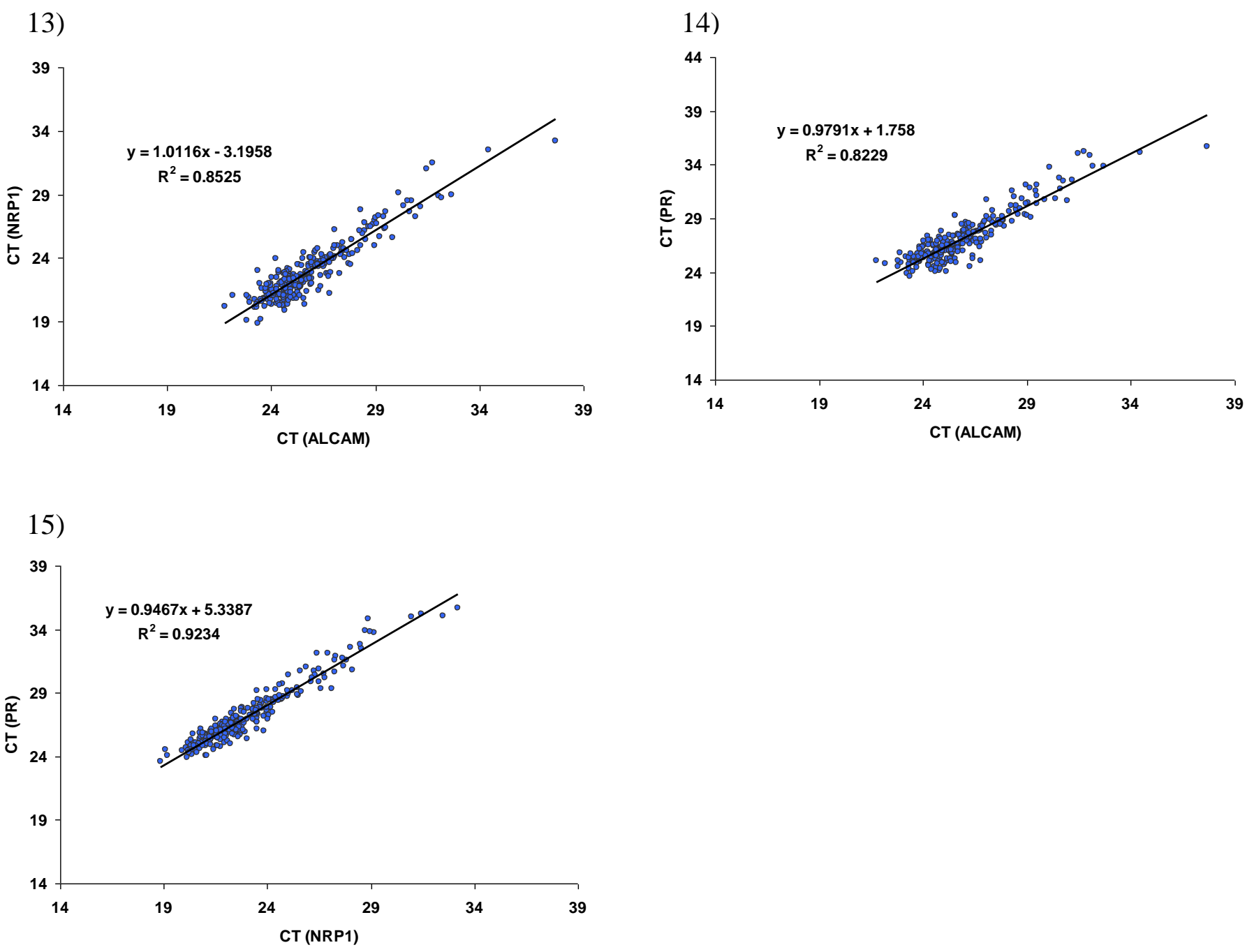

Figure 6A: Linear regressions of Cycle Threshold $\left(\mathrm{C}_{\mathrm{T}}\right)$ values of HAS2 and FSHR (1), HAS2 and ALCAM (2), HAS2 and VCAN (3), HAS2 and NRP1 (4), HAS2 and PR (5), VCAN and FSHR (6), VCAN and ALCAM (7), VCAN and NRPI (8), VCAN and PR (9), FSHR and ALCAM (10), FSHR and NRPI (11), FSHR and PR (12), ALCAM and NRP1 (13), ALCAM and $P R(14)$ and, NRP1 and $P R(15)$ mRNA in CC masses.

\section{Gene expression levels related to COC morphology}

Mean mRNA levels for ALCAM were significantly lower $(\mathrm{P}<0.05)$ in COC classified as light compared to those classified as dark, whilst the expressions of all other candidate genes were similar (Figure 6B). 


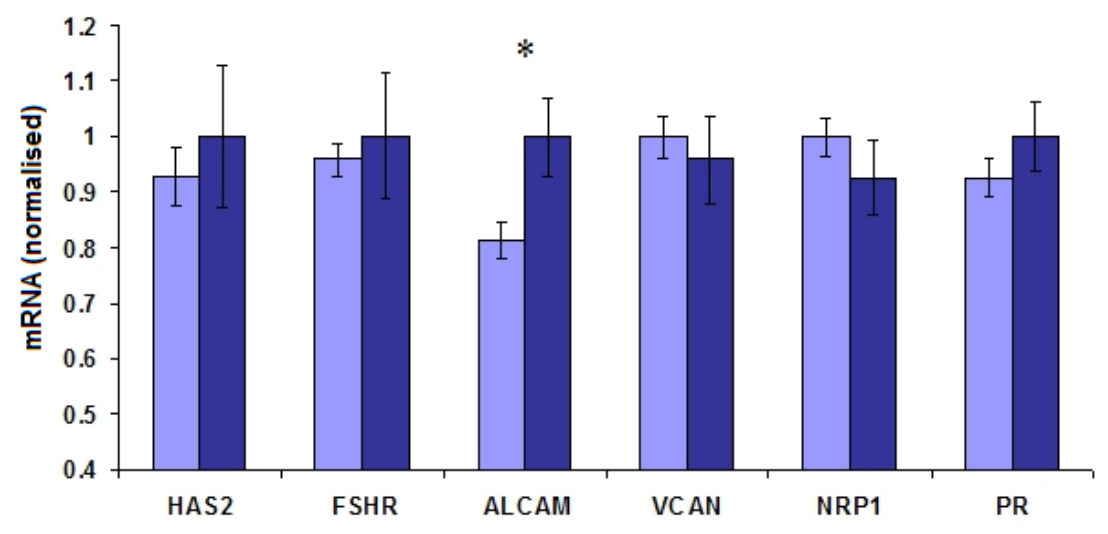

Figure 6B: Relative expression levels of candidate genes in CC associated with light (light blue histogram) and dark (dark blue histogram) COC. Values represent the means and SEM. $* \mathrm{P}<0.05, \mathrm{NS}$.

\section{Gene expression levels related to oocyte maturity}

Mean mRNA levels for VCAN and HAS2 were significantly lower (VCAN, $\mathrm{P}<0.0001$; HAS2, $\mathrm{P}<0.05)$, whilst those of $F S H R$ and $A L C A M$ tended to be lower $(\mathrm{P}=0.086, \mathrm{P}=0.054)$ in $\mathrm{CC}$ associated with oocytes that progressed to metaphase II (mature oocytes; MII) compared to those CC that remained immature (e.g. GV and MI). The mean NRPI and PR mRNA were not different in $\mathrm{CC}$ associated with mature or immature oocytes (Figure 6C).

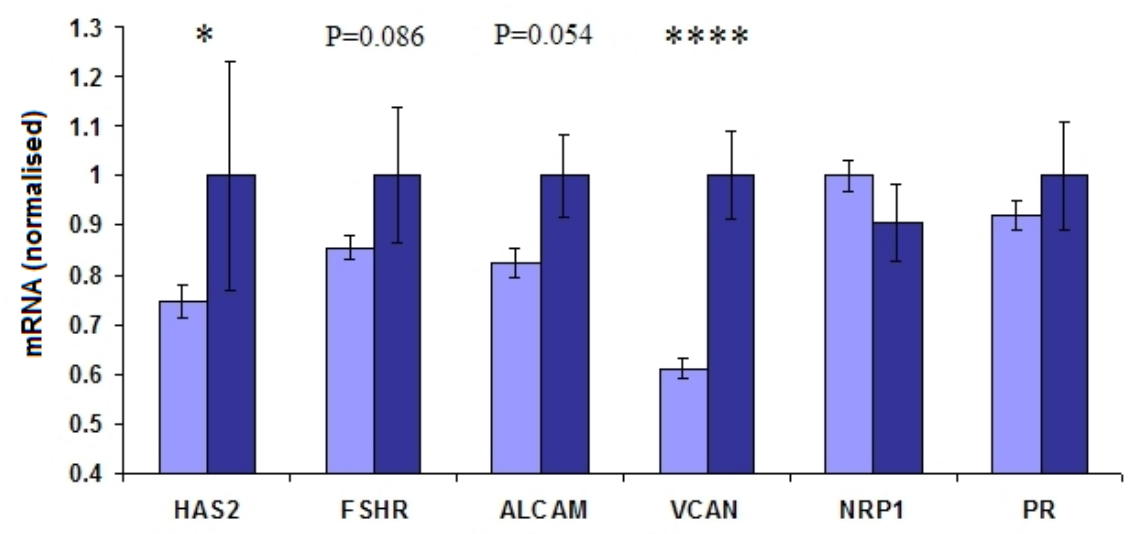

Figure 6C: Relative expression levels of candidate genes in CC associated with MII oocytes (light blue histogram) and with MI and GV oocytes (dark blue histogram). Values represent the means and SEM. $* * * * \mathrm{P}<0.0001, * \mathrm{P}<0.05$. 
Gene expression levels related to fertilization

Mean mRNA levels for all genes were similar in CC associated with oocytes that fertilized normally $(2 \mathrm{PN})$ compared with those that either failed to fertilise or were abnormal (i.e. 1PN, 3PN) (Figure 6D).

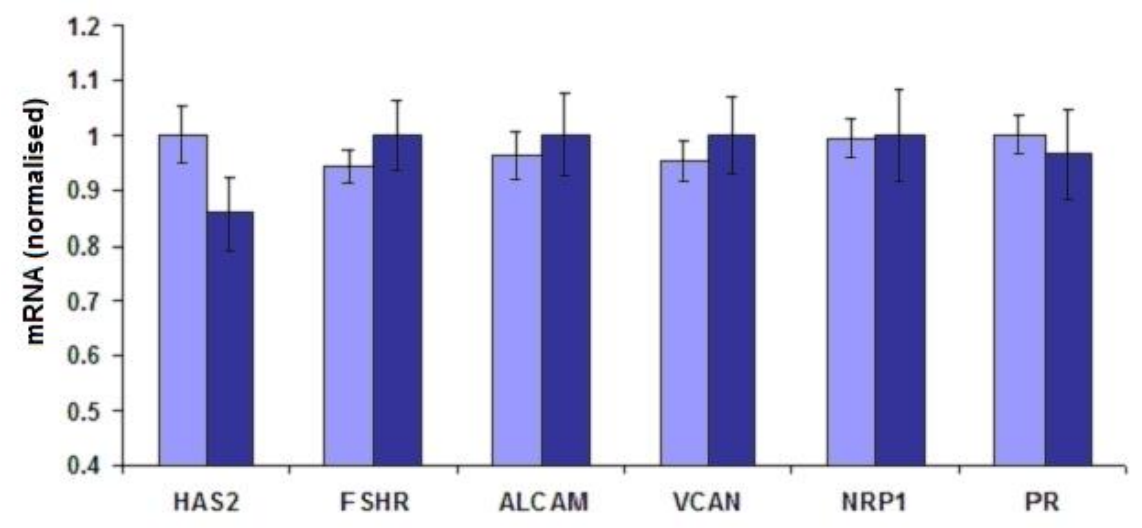

Figure 6D: Relative expression levels of candidate genes in CC associated with oocytes that fertilized normally (light blue histogram) and with oocytes that failed to fertilize or were abnormal (i.e. 1PN, 3PN) (dark blue histogram). NS.

Gene expression levels related to early embryonic development

Mean mRNA levels for all genes were similar in CC associated with oocytes that progressed to embryonic cleavage quickly ( $\geq 7$ cell on day 3 ) compared with those that resulted in slow embryonic cleavage ( $\leq 6$ cell on day 3 ) and arrested development (Figure $6 \mathrm{E}$ ).

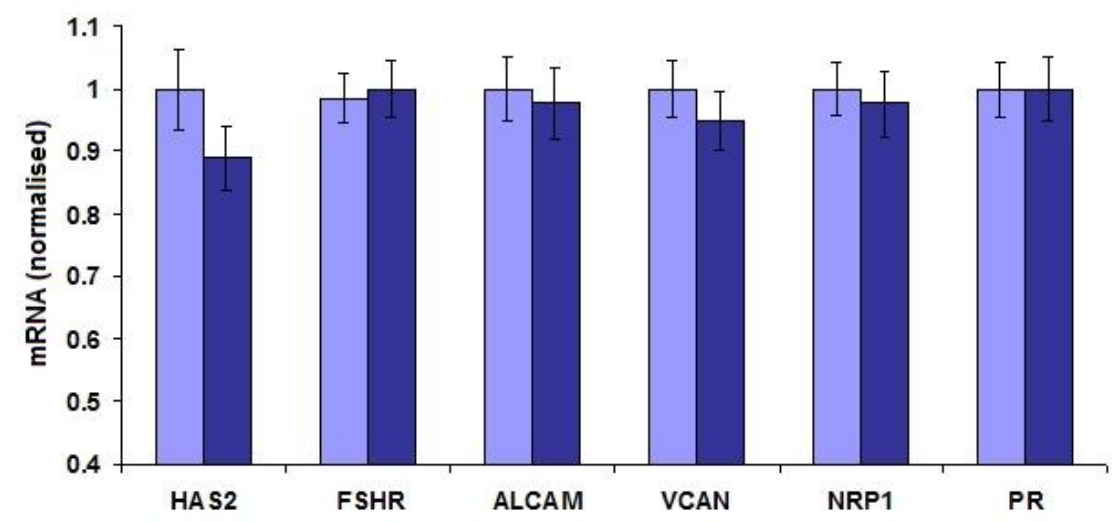

Figure 6E: Relative expression levels of candidate genes in CC associated with oocytes that progressed to embryonic cleavage quickly ( $\geq 7$ cell on day 3 ) (light blue histogram) and with 
oocytes that resulted in slow embryonic cleavage ( $\leq 6$ cell on day 3 ) and arrested development (dark blue histogram). NS.

\section{Gene expression levels related to blastocyst development}

Mean mRNA levels were significantly higher for HAS2 $(\mathrm{P}<0.05) ; V C A N(\mathrm{P}<0.005) ; P R$ $(\mathrm{P}<0.05)$ in $\mathrm{CC}$ associated with oocytes that progressed to a good quality blastocyst compared to $\mathrm{CC}$ associated with oocytes that failed to reach a viable blastocyst stage (Figure 6F). The expression levels for FSHR, ALCAM and NRPI did not differ in CC that formed good blastocysts or failed to reach that stage.

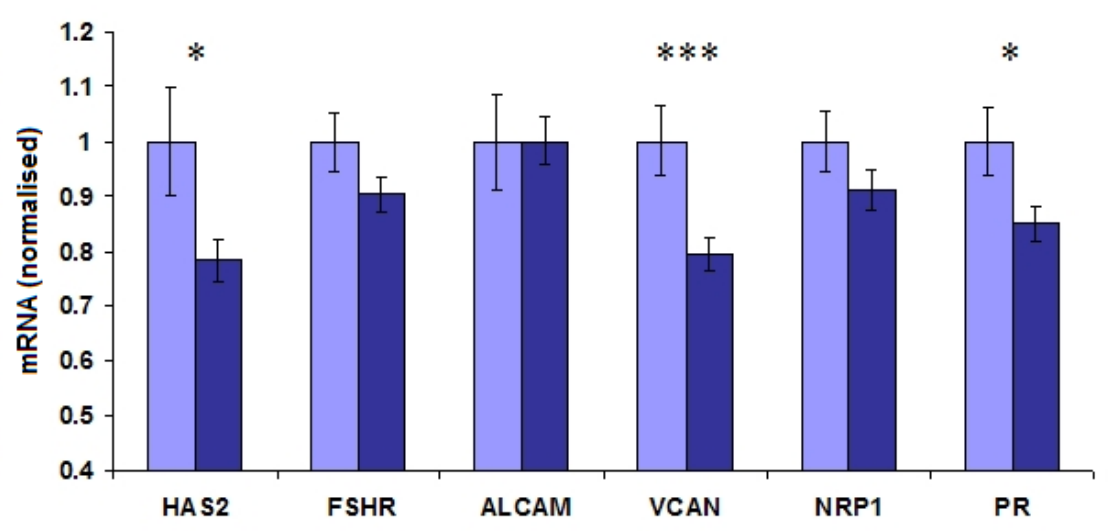

Figure 6F: Relative expression levels of candidate genes in $\mathrm{CC}$ associated with oocytes that progressed to good quality blastocyst (light blue histogram) and to oocytes with failed development (dark blue histogram). Values represent the means and SEM. $* * * \mathrm{P}<0.005$, $* \mathrm{P}<0.05, \mathrm{NS}$.

\section{Gene expression levels related to pregnancy outcome}

Mean mRNA levels for VCAN were significantly higher $(\mathrm{P}<0.05)$ and HAS2 tended to be higher $(\mathrm{P}=0.079)$ in $\mathrm{CC}$ associated with oocytes that progressed to a positive hCG level compared to levels in $\mathrm{CC}$ associated with negative outcome (data of $\mathrm{CC}$ associated with oocytes that progressed to good quality blastocysts and were frozen, were not included in this comparison). Mean mRNA levels for all other genes were not different for the same comparisons (Figure 6G). 


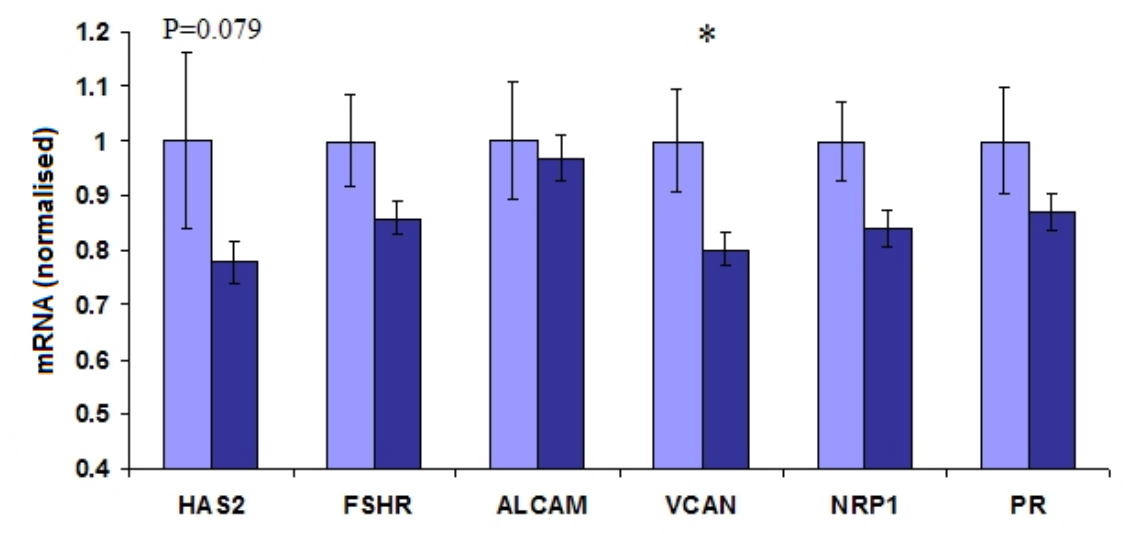

Figure 6G: Relative expression levels of candidate genes in CC associated with oocytes that progressed to positive pregnancy (light blue histogram) and with oocytes associates with failed development (dark blue histogram). Values represent the means and SEM. $* \mathrm{P}<0.05$, NS.

Gene expression levels related to live birth outcome

Mean mRNA levels for VCAN were significantly higher $(\mathrm{P}<0.05)$ in $\mathrm{CC}$ associated with oocytes that resulted in a healthy term live birth outcome compared to $\mathrm{CC}$ associated with negative outcome (data of CC associated with oocytes that progressed to good quality blastocysts and were frozen, were not included in this comparison). Mean mRNA levels for all other genes were similar in the same comparison (Figure $6 \mathrm{H}$ ).

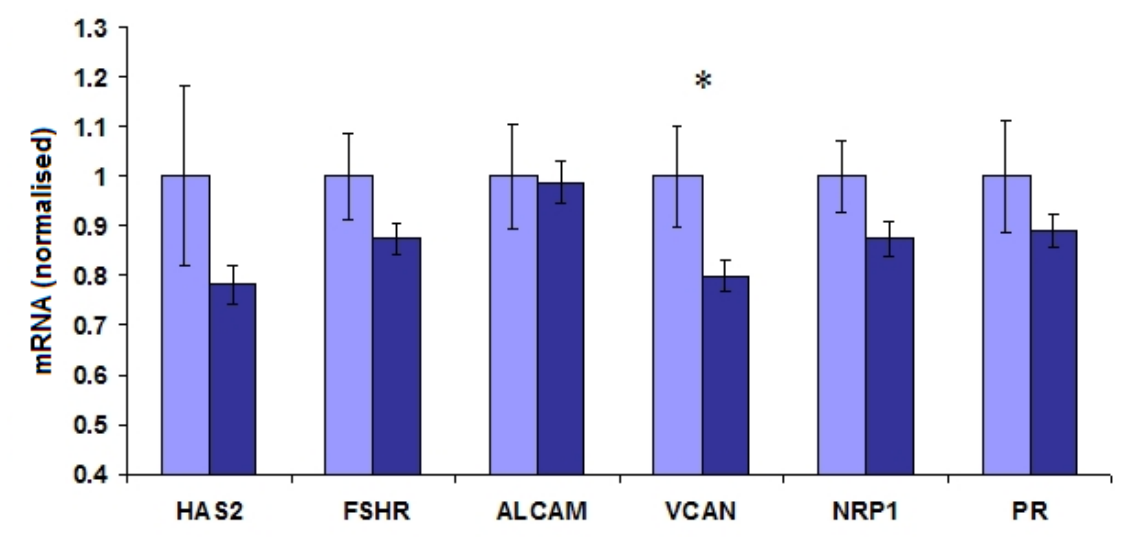

Figure 6H: Relative expression levels of candidate genes in $\mathrm{CC}$ associated with oocytes that resulted in a healthy term live birth (light blue histogram) and to oocytes associated with failed development (dark blue histogram). Values represent the means and SEM. ${ }^{*} \mathrm{P}<0.05$, NS. 


\subsection{Discussion}

The present study is the first to investigate the gene expression levels of multiple candidate genes in individual human CC samples in relation to blastocyst development and pregnancy outcome by multiplex TaqMan QPCR.

The major finding in this study was the highly significant increase in mRNA expression levels of HAS2, VCAN and $P R$ in $\mathrm{CC}$ from oocytes that developed into embryos that progressed to pregnancy and/or blastocysts that were selected for freezing compared to the expression levels in $\mathrm{CC}$ associated with oocytes with failed development. Moreover, the present study is the first to report a significant increase in mRNA expression levels of VCAN in $\mathrm{CC}$ from oocytes that resulted in a live birth relative to the total number of MII oocytes available after follicle aspiration.

From a review of twelve previous reports on the relationship between $\mathrm{CC}$ gene expression levels and human embryological and pregnancy outcomes in which numerous genes have been investigated (e.g. GREM1, HAS2, PTGS1, PTGS2, PTX3, TNFAIP6, PR, STAR, COX2, CX43, AREG, SCD1, SCD5, BCL2L11, PCK1, NFIB, ALCAM, SDC4, VCAN, CALM2), no previous study has found a statistically significant correlation between mRNA expression levels in $\mathrm{CC}$ and blastocyst and pregnancy outcome of the associated oocyte within the total number of MII oocytes available following oocyte retrieval (McKenzie et al. 2004; Hasegawa et al. 2005; Zhang et al. 2005; Cillo et al. 2007; Feuerstein et al. 2007; Assou et al. 2008; Hamel et al. 2008; van Montfoort et al. 2008; Anderson et al. 2009; Adriaenssens et al. 2010; Gebhardt et al. 2011; Wathlet et al. 2011).

In human IVF, where the majority of MII oocytes appear morphologically identical, the ideal set of oocyte quality markers would be those able to predict embryo potential from all MII oocytes available. Our study is the first to accurately determine the mRNA expression levels of HAS2, VCAN and $P R$ in individual human $\mathrm{CC}$ masses associated with oocytes that developed into good quality blastocysts (embryos that progressed to pregnancy and blastocyst frozen for subsequent use) following IVF with respect to the number of MII oocytes recovered. Previous QPCR studies reported (1) lower $(\mathrm{P}<0.05)$ mRNA expression levels for STAR, COX2, CX43, AREG, SCD1, SCD5 genes in CC associated with oocytes that developed to blastocyst compared with oocytes that did not become blastocyst (Day 5 or Day 
6 assessment) (Feuerstein et al. 2007) and (2) higher SDC4 ( $\mathrm{P}<0.05), A L C A M(\mathrm{P}<0.01)$ and lower VCAN ( $\mathrm{P}<0.01)$ levels of oocytes that developed to blastocyst on Day 5 (Wathlet et al. 2011). However, neither of these studies reported statistical differences in relation to the total number of MII oocytes recovered. Perhaps this may be the underlying reason why these authors found that higher ALCAM and lower VCAN mRNA levels in CC were associated with blastocyst development, whilst our results show that blastocyst formation is correlated to higher CC mRNA levels of VCAN, whilst mRNA levels of ALCAM are not correlated to good blastocyst development.

Similarly, no previous study has reported statistical differences in CC-derived gene expression levels in regards to pregnancy outcome when considering the total number of MII oocytes recovered. One previous study reported higher $(\mathrm{P}<0.01)$ levels of CC-derived PTX3 levels associated with 8-cell (Day 3) embryos that progressed to pregnancy compared with oocytes with failed fertilization, but no significant differences compared with 8-cell (Day 3) embryos with failed implantation (Zhang et al. 2005). Another study reported no significant differences in the expression levels of HAS2, BDNF, GREM1, PTGS2, TNFAIP6 and PTX3 genes, in relation to pregnancy. However, most of the women involved in the aforementioned study had double embryo-transfers thereby preventing a clear analysis of embryo outcome (Anderson et al. 2009). A recent study using antagonist rFSH, but not agonist HP-hMG, stimulated IVF cycles reported that CC mRNA levels of SDC4, VCAN ( $\mathrm{P}<0.001), A L C A M$, TRPM7, ITPKA $(\mathrm{P}<0.01)$, GREM1 and PTGS2 $(\mathrm{P}<0.05)$ were higher in CC associated with transferred embryos that progressed to pregnancy compared with $\mathrm{CC}$ associated with transferred embryos with failed implantation (Wathlet et al. 2011). In a similar analysis it was confirmed that VCAN and PTGS2 levels are higher in $\mathrm{CC}$ associated with transferred embryos that resulted in live births compared to $\mathrm{CC}$ associated with transferred embryos with failed implantation (Gebhardt et al. 2011). In the present study, comparing data obtained from the small number of oocytes that resulted in an embryo transfer and culminated into a live birth against the data obtained from the large number of oocytes that resulted in negative outcome, VCAN expression levels were significantly higher and HAS2 expression levels were more frequently higher in $\mathrm{CC}$ of oocytes associated with live birth (data from $\mathrm{CC}$ associated with oocytes that resulted in good quality blastocysts and were frozen for subsequent use were left out of this analysis, as outcome of those embryos is not known yet). 
It is evident that the expression levels of HAS2 and VCAN are good predicators of oocyte maturity. This is in line with previous reports by Adriaenssens et al (2010) and Wathlet et al (2011) who showed that VCAN expression in CC was negatively correlated with oocyte maturity. With respect to previous studies of HAS2 in relation of oocyte maturity, the results have generally been variable (McKenzie et al. 2004; Anderson et al. 2009; Adriaenssens et al. 2010). Overall, the mRNA levels of the eight genes presented in this study are poor predictors of successful fertilization and early embryo cleavage. These findings are consistent with those in previous reports (McKenzie et al. 2004; Hasegawa et al. 2005; Cillo et al. 2007; Anderson et al. 2009; Adriaenssens et al. 2010; Wathlet et al. 2011).

In conclusion, the results from this study suggest that the mRNA levels of VCAN, HAS2 and $P R$ in $\mathrm{CC}$ have the highest potential to serve as molecular markers for key indicators of oocyte development, namely blastocyst formation and live birth outcome. 


\section{Chapter 7 RANKING OF INDIVIDUAL COC ACCORDING TO CC GENE EXPRESSION LEVELS AND CC NUMBERS AND THE SELECTION OF OOCYTES WITH HIGH DEVELOPMENTAL POTENTIAL}

\subsection{Introduction}

Stimulated IVF cycles result in significantly higher pregnancy rates compared to natural IVF cycles (Pelinck et al. 2002), however live birth rates arising from the numbers of oocytes recovered is very low ( 7\%) (Li et al. 2008; Patrizio and Sakkas 2009). This is perhaps not surprising as most follicles within the ovary have different endocrine and nutrient microenvironments, as well as somatic cells at different stages of maturation (McNatty et al. 2010). Moreover, studies in sheep have shown that super-ovulation regimes do not effectively override the hierarchical pattern of follicular development, where most, if not all, antral follicles within an ovary respond individually and not identically to gonadotrophins (McNatty et al. 1990; McNatty et al. 1999; McNatty et al. 2010). Currently, there are no reliable noninvasive methods which enable the selection of good quality oocytes to be distinguished from poor quality oocytes following their recovery in stimulated IVF cycles (Rienzi et al. 2011).

Two important questions are still waiting to be answered for human gonadotrophin-stimulated IVF cycles. The first is "Can we develop reliable markers for the selection of good quality oocytes following the stimulation of multiple pre-ovulatory follicles?" and the second is "What is the level of synchronization of the MII oocytes collected following gonadotrophin stimulation?" To answer these questions, the mRNA levels of six candidate genes (HAS2, FSHR, ALCAM, VCAN, NRP1, PR) in CC, and numbers of CC from individual COC using multiplex QPCR techniques were assessed: (1) in relation to oocyte development (as indicated by blastocyst development and pregnancy) and; (2) the extent to which the mRNA levels in $\mathrm{CC}$ are consistently uniform amongst MII oocytes. 


\subsection{Materials and Methods}

From the 25 women recruited in Gp 1 in which COC were collected, the CC masses were removed from their associated oocytes and processed individually by TaqMan QPCR for expression levels of six candidate genes (HAS2, FSHR, ALCAM, VCAN, NRP1, PR). The expression levels of a housekeeper gene (RPL19) were also measured for correction of candidate gene expression levels and for determining the number of $\mathrm{CC}$ present for each $\mathrm{CC}$ mass. The methodologies including hormonal stimulations, COC collections, embryo culture and transfer, luteal support, calculation of $\mathrm{CC}$ numbers and quantification of target mRNA levels were all performed as previously described (Chapter 2-4 and 6, Appendix 2.1-2.2 and 3.1-3.3).

\section{Oocyte selection by random selection}

The probability of selecting at least one good quality oocyte (measured by good blastocyst development and pregnancy) from a random selection of one or three COC from the total number of MII oocytes recovered from one woman was calculated according to the formula:

$$
\mathrm{P}=1-\left(\mathrm{x}^{\mathrm{neg}} / \mathrm{x}^{\mathrm{tot}}\right)^{n}
$$

where $\mathrm{P}=$ probability, $\mathrm{x}^{\text {neg }}=$ number of oocytes with negative outcome, $\mathrm{x}^{\text {tot }}=$ number of total oocytes collected, $n=$ number of COC selected. From these probability values, the mean probability \pm SEM of selecting a good quality oocyte was calculated for Gp 1 women.

\section{Ranking analysis of CC numbers in individual COC}

The number of CC and 2xStDev were calculated from the repeated measurement $(\mathrm{N}=68)$ of the reference gene of the calibrator sample present in every QPCR run. Following the calculation of $\mathrm{CC}$ numbers for each $\mathrm{COC}$, these were ranked with 1 being indicated for the lowest and thereafter sequentially to the highest. Individual COC within each patient were considered statistically different from each other if the number of CC differed by 2xStDev. Samples that showed similar CC numbers with two or more separate ranks (i.e. 1 and 2), were grouped in the lower ranks (i.e. 1) to simplify the analysis.

The proportion of individual COC with different CC numbers (i.e. different ranks) was calculated for each woman and the mean proportion \pm SEM was then determined. 


\section{Ranking of gene expression in individual COC}

For the individual CC samples, the $2^{-\Delta \mathrm{CT}}$ values were calculated for each of the six candidate genes as described in the General Methodology (Chapter 2). Similarly, the $2 \mathrm{xStDev}$ of $2^{-\Delta \mathrm{CT}}$ values were calculated for each gene from the repeated measurement of the calibrator sample. Individual COC samples from each woman were then ranked from lowest to highest with respect to gene expression levels $\left(2^{-\Delta \mathrm{CT}}\right)$. The individual values within each patient were considered statistically different from one another if their expression values $\left(2^{-\triangle \mathrm{CT}}\right)$ differed by $2 \mathrm{xStDev}$. In each patient, the gene expression level with the lowest $2^{-\mathrm{CCT}}$ value was ranked 1 , whilst those with significantly higher $2^{-\Delta \mathrm{CT}}$ values were ranked 2 , and those with significantly higher $2^{-\Delta \mathrm{CT}}$ values compared with rank 2 were ranked 3 , etc. Samples that showed similar gene expression $\left(2^{-\Delta \mathrm{CT}}\right)$ values to two or more separate ranks (i.e. 1 and 2 ), were grouped in the lower ranks (i.e. 1) to simplify the analysis.

The proportion of individual COC with different gene expression levels (i.e. different ranks) was calculated for each gene within each patient and a mean proportion \pm SEM was calculated for the women in Gp 1. Finally, the mean proportion \pm SEM of COC showing significantly different expression levels for each of the genes was then determined.

\section{Ranking of individual COC for oocyte selection}

The COC from each patient were ranked according to $\mathrm{CC}$ numbers and mRNA expression levels for each of the six candidate genes. These COC ranks were then analysed for each individual parameter, or collectively for groups of parameters, in relation to the outcome of their associated oocyte (good blastocyst and live birth) to determine potential indicators of oocyte quality.

To determine the most successful method for selecting at least one good quality oocyte, all selection methods i.e. 1) using all COC recovered, 2) randomly selecting one or three of the recovered $\mathrm{COC}$ and 3) selecting one or three of the recovered $\mathrm{COC}$ according to ranking (see previous paragraph) were compared by one- and/or two-way ANOVA (SPSS).

To determine the level of heterogeneity of MII oocytes, the level of synchronization of the number of CC and levels of mRNA (HAS2, FSHR, ALCAM, VCAN, NRP1, PR) in individual COC was assessed. The level of heterogeneity of MII oocytes was further analysed in regards to the level of synchronization of the pre-ovulatory follicles ( $\geq 14 \mathrm{~mm}$ ). 


\subsection{Results}

\section{Follicle diameters on the day of hCG treatment}

From the $11 \pm 0.5$ follicles of $\geq 14 \mathrm{~mm}$ in size that were present on the day of administration of hCG, $5.0 \pm 0.5(43 \pm 3 \%)$ were of identical diameter (data not shown).

\section{Ranking analysis of individual COC}

Ranking analysis of individual COC based on CC numbers

Of the 232 oocytes that developed to the MII stage of maturation, $227(9.1 \pm 0.7 /$ patient, range: 3-15) were associated with CC samples that had measurable RPL19 levels from which CC numbers were estimated. Of these, $61 \pm 3 \%$ (5.2 \pm 0.3/patient) [range: $40 \%$ (Patient \#9) to $100 \%$ (Patient \#1) or 6/15 to 3/3] COC were significantly different in numbers of CC within the total number of COC for each woman (Figure 7A). 
1)

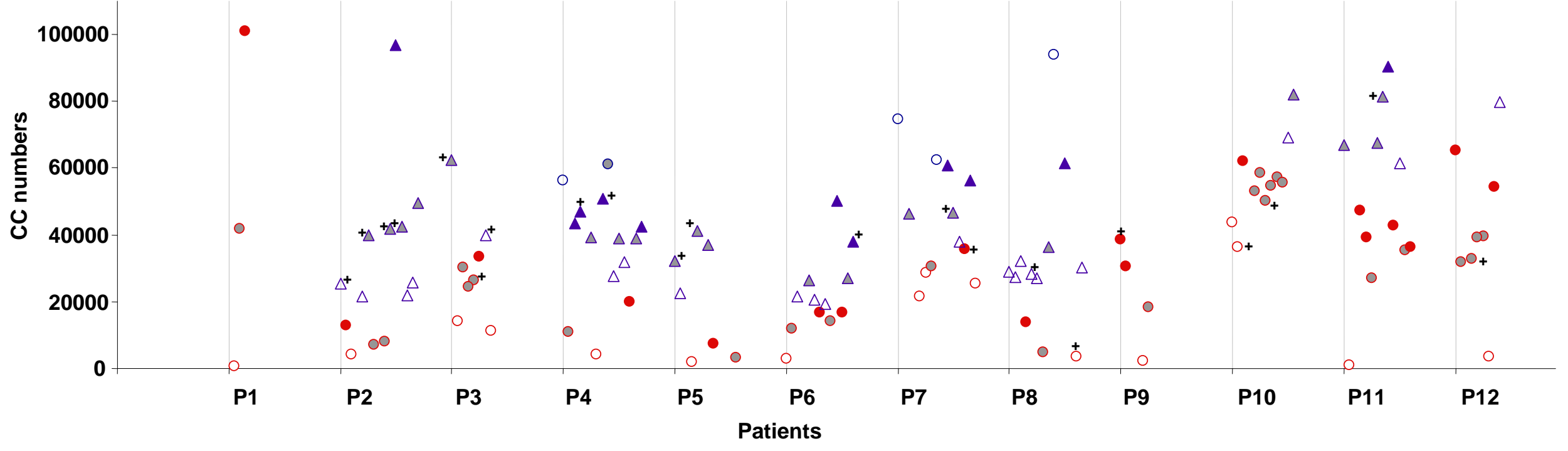

2)

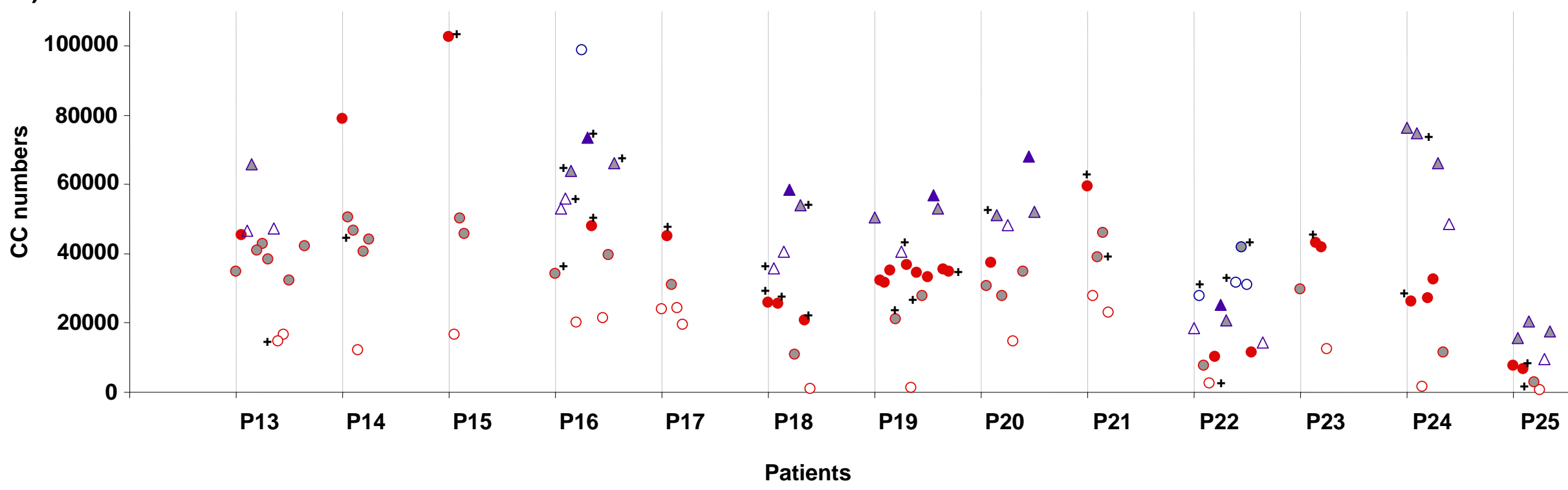


Figure 7A: Rankings of individual COC within each patient, in regards to estimated CC numbers for all women in this study. Each patient (P1-P25) is indicated on the $X$ axis and the individual COC belonging to each patient are separated by grey vertical lines. Individual COC are plotted from left to right according to their numerical order assigned at the time of egg collection, where COC \#1 is plotted on the left vertical line. “+” marks are associated to dots (oocytes) with a positive outcome (blastocyst development and/or positive pregnancy). Different dots denote different ranks $(\circ=1, \diamond=2, \bullet=3, \Delta=4, \Delta=5, \Delta=6, \circ=7, \diamond=8$ and, $\bullet=9)$.

\section{Ranking analysis of individual COC based on the expression of a single gene}

Of the 227 (9.1 \pm 0.7/patient, range: 3-15) MII oocytes associated with CC samples that had measurable levels of mRNA, the proportions of $\mathrm{CC}$ masses with different pattern of gene expression within the total number of $\mathrm{CC}$ masses per woman were: $55 \pm 2 \%[4.8 \pm 0.4$ /patient, range: $33 \%$ or $4 / 12$ (Patient \#7) to $83 \%$ or $5 / 6$ (Patient \#14)] for HAS2; $55 \pm 4 \%$ [4.8 \pm 0.4/patient, range: $20 \%$ or $1 / 5$ (Patient \#23) to $100 \%$ or $4 / 4$ (Patient \#9 and \#15)] for FSHR; $69 \pm$ $3 \%$ [5.9 $\pm 0.4 /$ patient, range: $38 \%$ or $3 / 8$ (Patient \#15) to $100 \%$ or $3 / 3$ (Patient \#1) and $4 / 4$ (Patient \#9 and \#15)] for ALCAM; $61 \pm 3 \%$ [5.2 \pm 0.3/patient, ranage: 33\% or 4/12 (Patient \#13) to $100 \%$ or $3 / 3$ (Patient \#1) and 4/4 (Patient \#9)] for VCAN: $56 \pm 3 \%$ [5.0 \pm 0.4/patient, range: $33 \%$ or 3/9 (Patient \#24) to $100 \%$ or 3/3 (Patient \#1)] for NRP1 and: $62 \pm 4 \%$ [5.2 \pm 0.4/patient, range: $36 \%$ or $4 / 11$ (Patient \#10) to $100 \%$ or $3 / 3$ (Patient \#1) and 4/4 (Patient \#9 and \#15)] for PR (Figure 7B). 


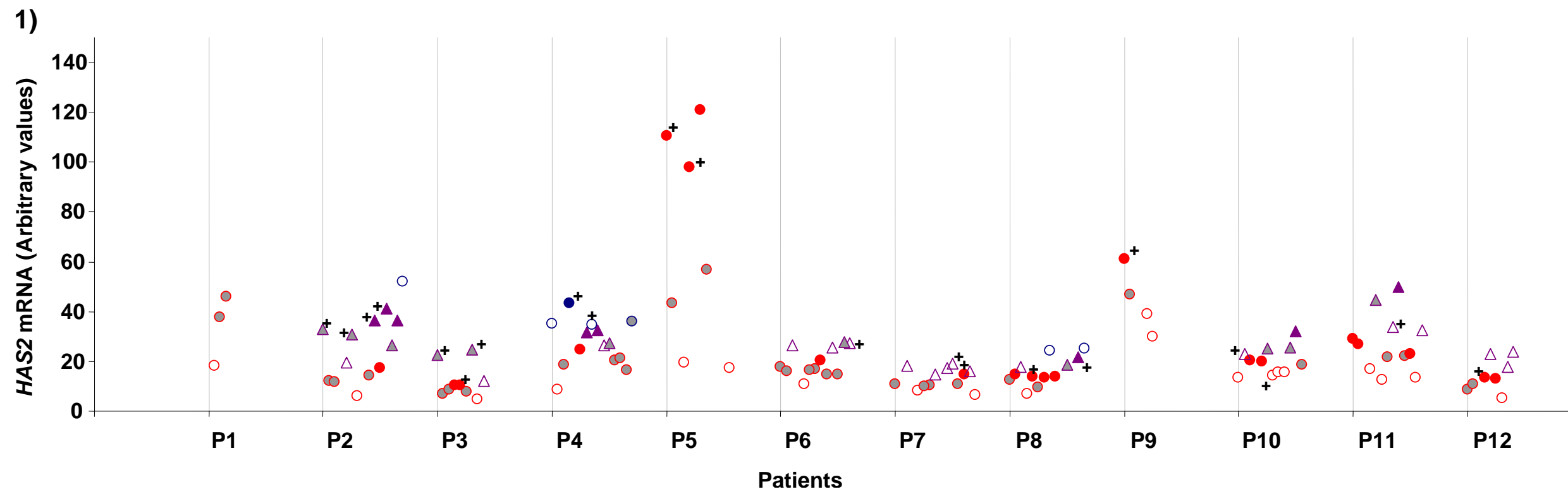

2)

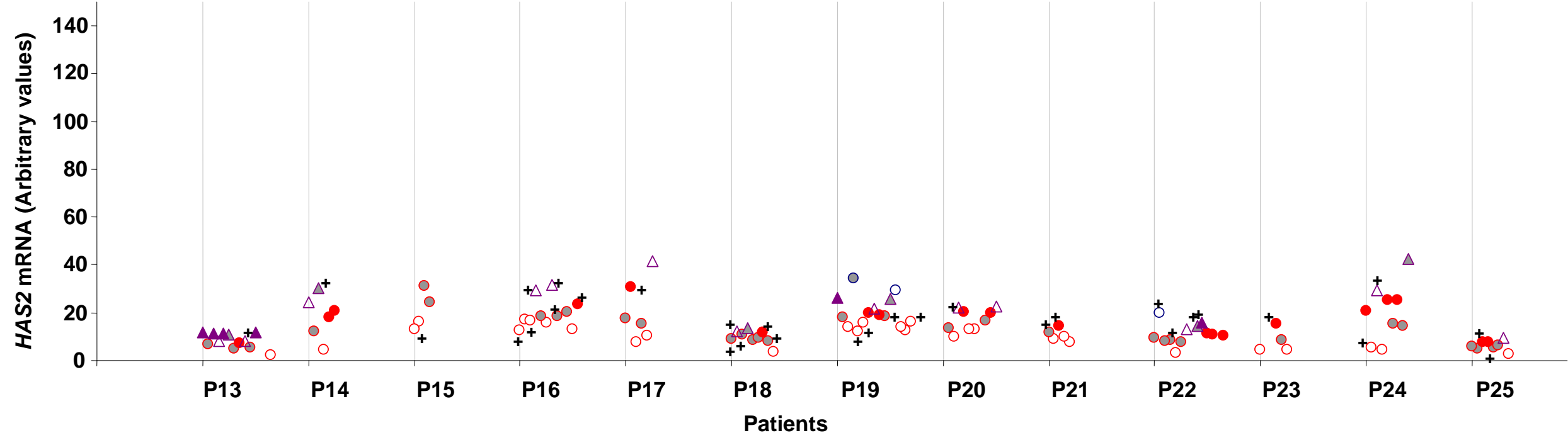


3)

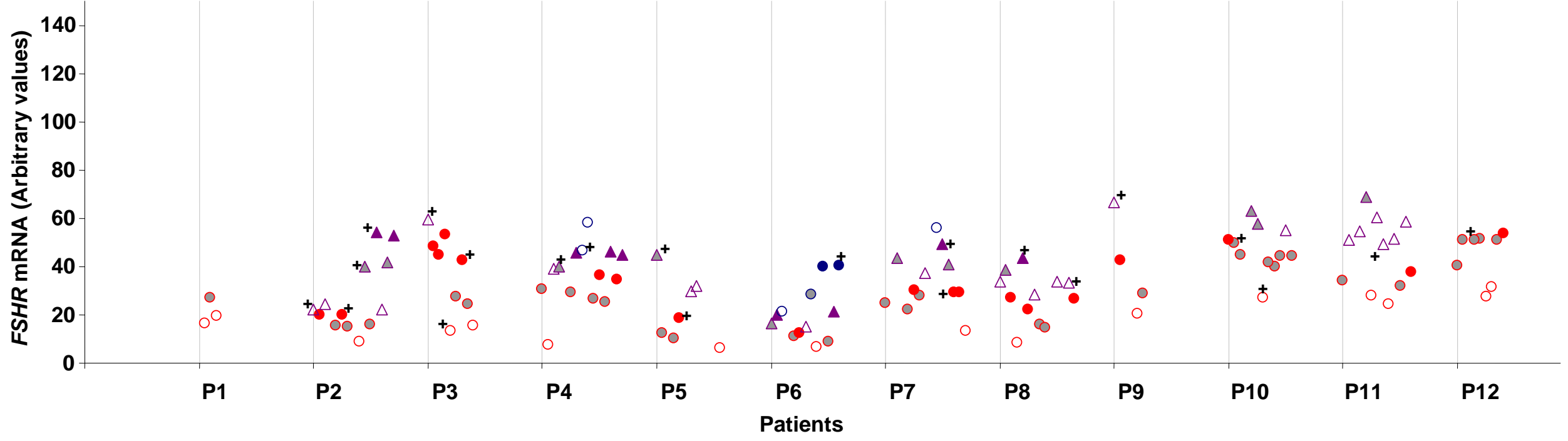

4)

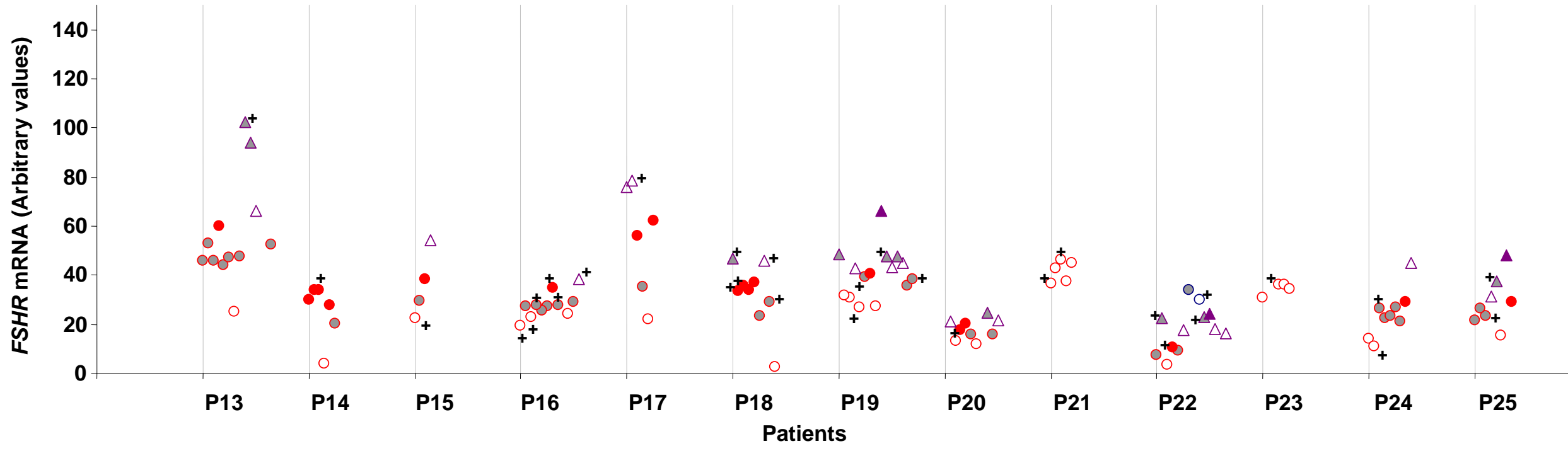


5)

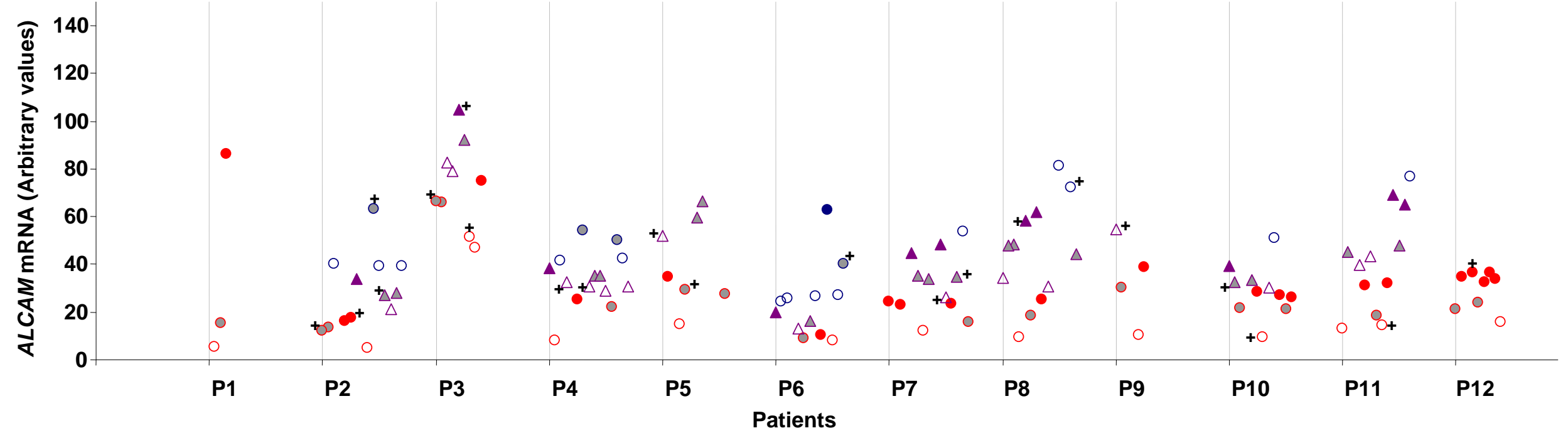

6)

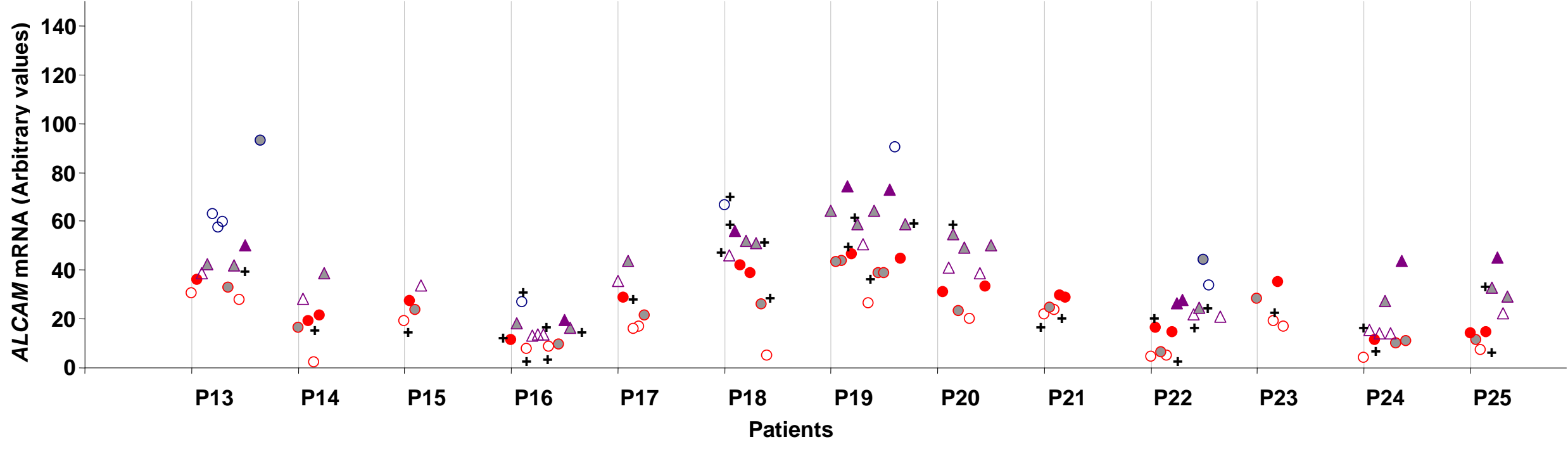


7)

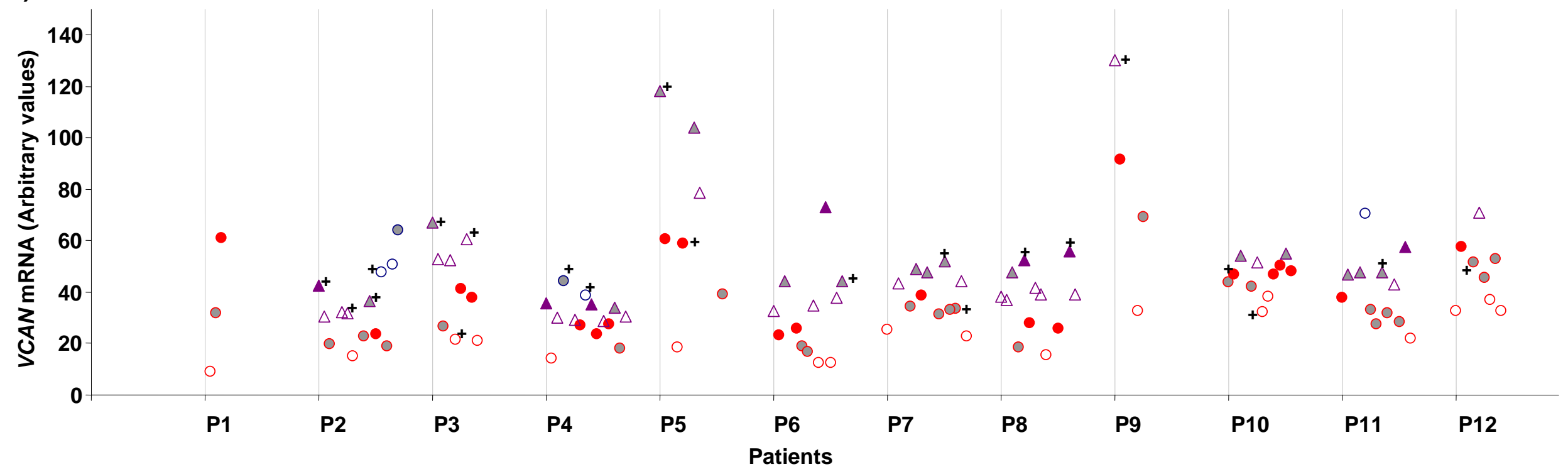

8)

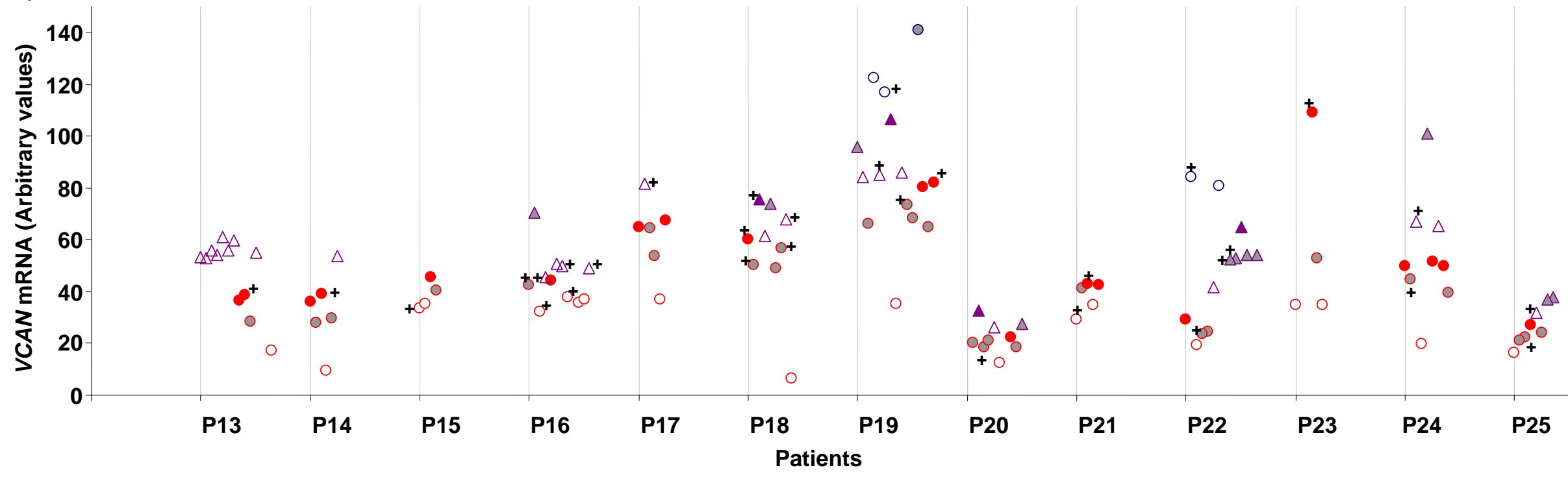


9)

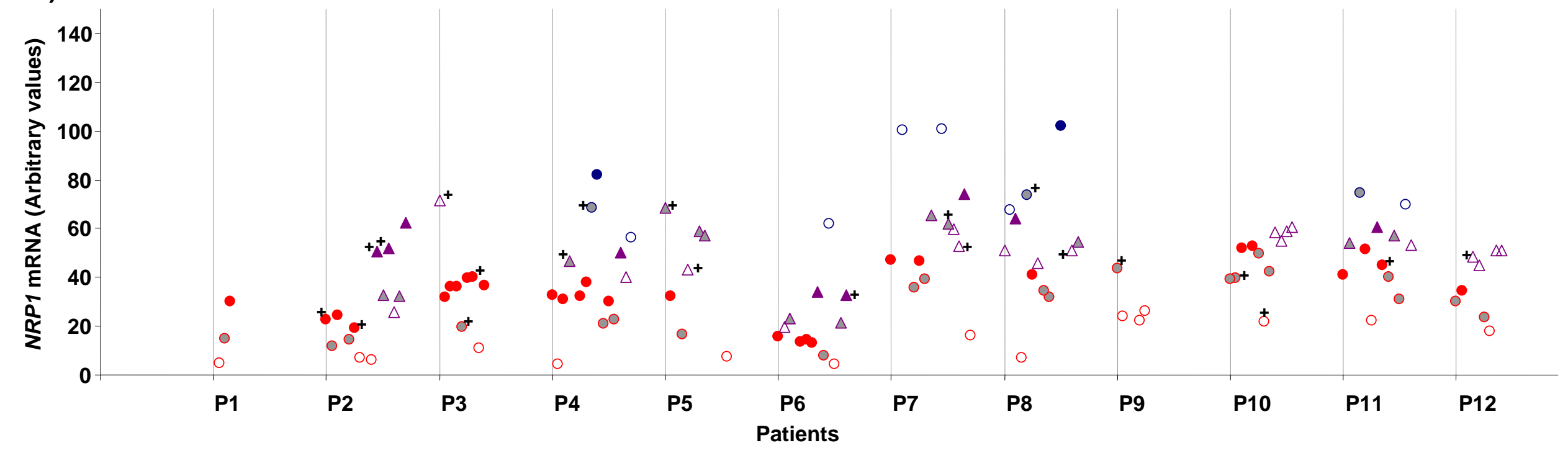

10)

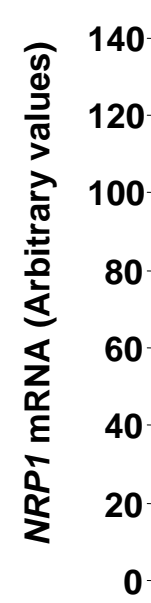

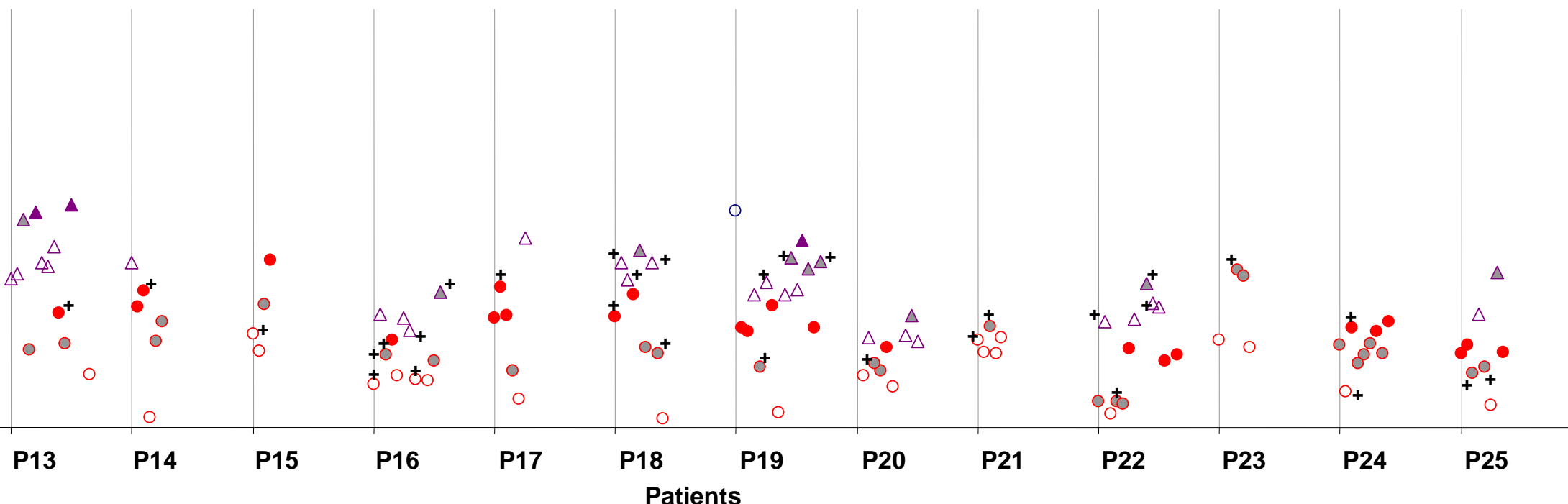


11)

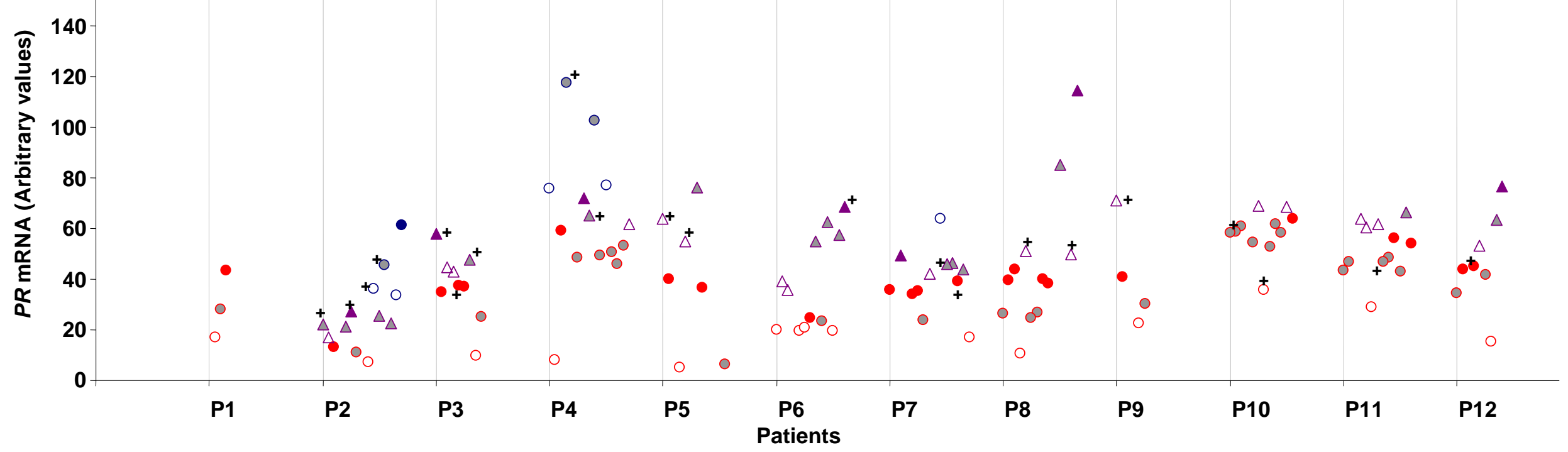

12)

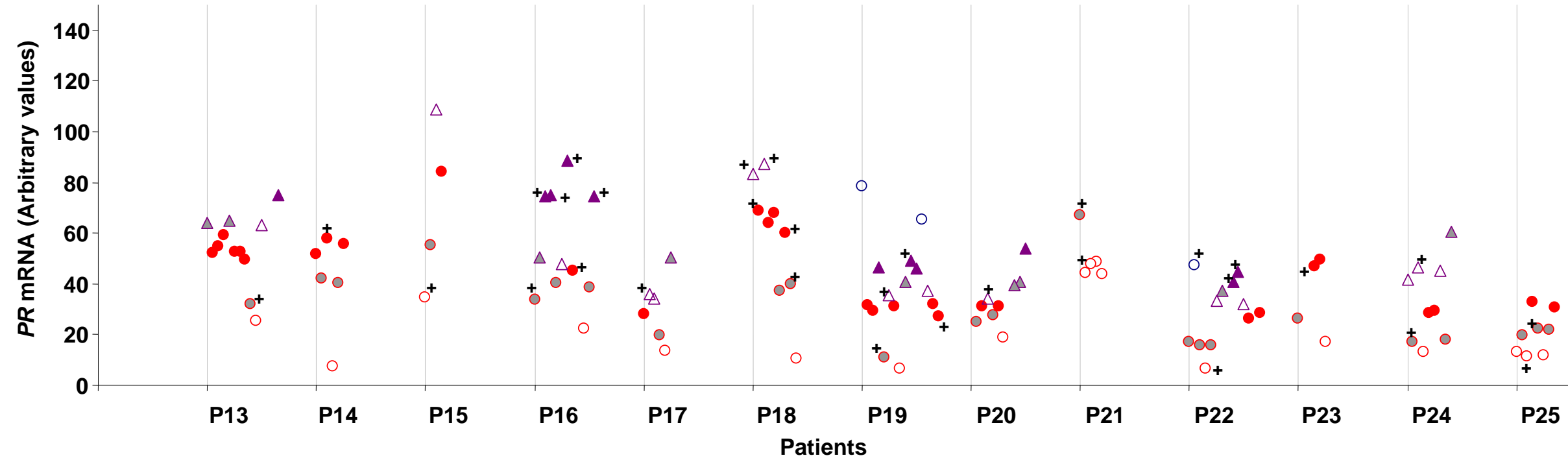


Figure 7B: Rankings of individual COC from each patient, with respect to the expression levels of six candidate genes. Each patient (P1-P25) is represented on the $\mathrm{X}$ axis, where all the COC for each patient are separated by the grey vertical lines. Individual COC are plotted from left to right according to their numerical order assigned at the time of egg collection, where COC \#1 is plotted on the left vertical line. "+" marks are associated to dots (oocytes) with a positive outcome (blastocyst development and/or positive pregnancy). Different dots denote different ranks $(\circ=1, \diamond=2, \bullet=3, \Delta=4, \Delta=5, \Delta=6, \circ=7, \diamond=8$ and, $\bullet=9)$. Gene expression is presented in $2^{-}$ ${ }^{\Delta \mathrm{CT}}$ values (HAS2: 2000x $2^{-\Delta \mathrm{CT}}$, FSHR: 10000x $2^{-\Delta \mathrm{CT}}$, ALCAM: 5000x $2^{-\Delta \mathrm{CT}}$, VCAN: 100x $2^{-\Delta \mathrm{CT}}$, NRP1: 500x $2^{-\Delta \mathrm{CT}}$ and, PR: $\left.10000 \times 2^{-\Delta \mathrm{CT}}\right)$.

\section{Ranking analysis of individual COC based on the expression of six genes}

The proportion of COC with significantly different expression levels for at least one of the six candidate genes was $99.7 \%$. Of the 227 COC analysed, only two (COC \#3 and \#14 from Patient \#19) shared the same gene expression levels for all six genes (although were ranked differently in the analyses according to FSHR and $A L C A M$ ). These two individual COC also shared the same number of $\mathrm{CC}$, and both were associated with oocytes with a negative outcome (Figure 7C and Appendix 7). 


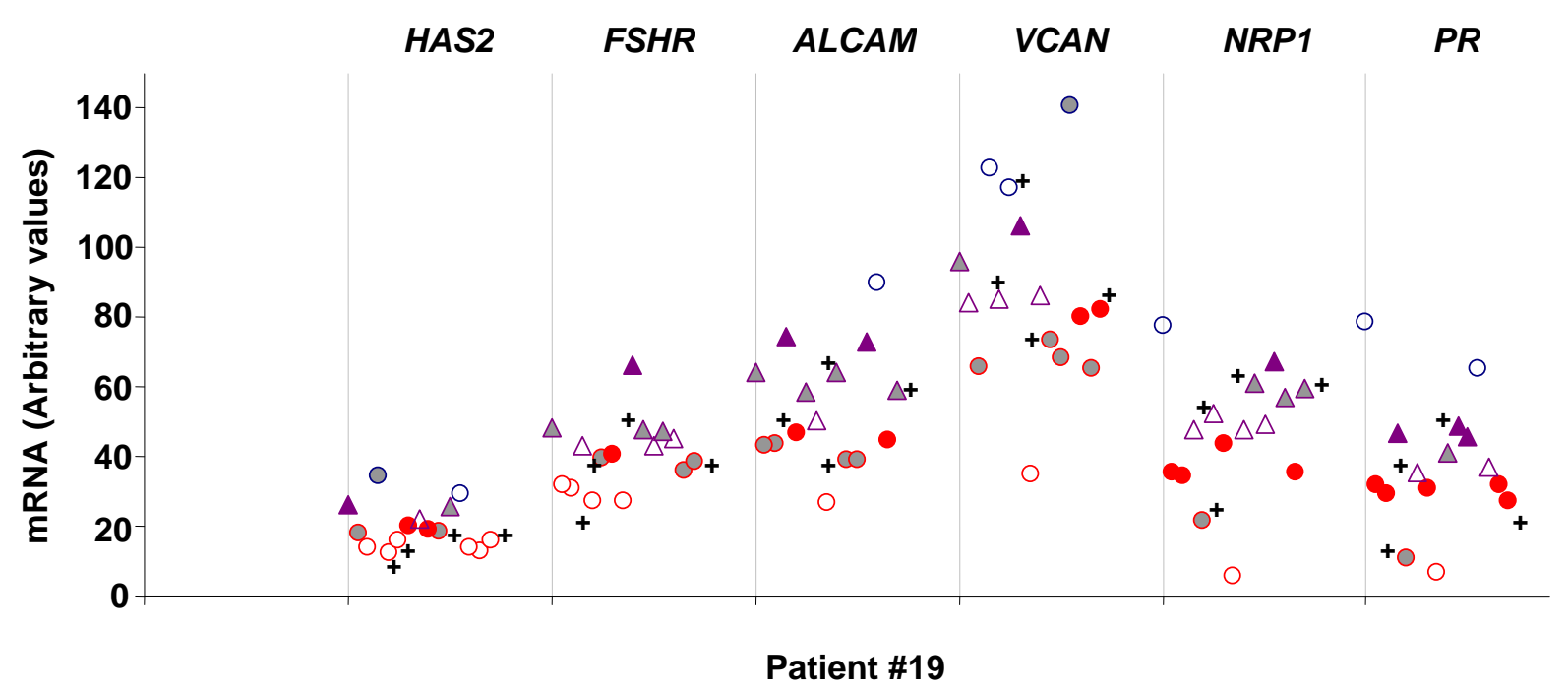

Figure 7C: Rankings of individual COC according to gene expression for patient \# 19. Individual $\mathrm{COC}$ are spread along the $\mathrm{X}$ axis for each candidate gene (HAS2, FSHR, ALCAM, VCAN, NRP1 and $P R$ ) from left to right according to their numerical order assigned at the time of egg collection. Expression results for each gene are separated by grey vertical lines. "+" marks are associated to dots (oocytes) with a positive outcome (blastocyst development and/or positive pregnancy). Different dots denote different ranks ( $\circ=1, \diamond=2, \bullet=3, \Delta=4, \Delta=5, \Delta=6, \circ=7, \circ=8$ and, $\bullet=9$ ). Gene expression is presented in $2^{-\Delta \mathrm{CT}}$ values (HAS2: 2000x $2^{-\triangle \mathrm{CT}}$, FSHR: 10000x $2^{-}$ ${ }^{\Delta \mathrm{CT}}$, ALCAM: 5000x $2^{-\Delta \mathrm{CT}}$, VCAN: 100x $2^{-\Delta \mathrm{CT}}$, NRP1: 500x $2^{-\Delta \mathrm{CT}}$ and, PR: 10000x $2^{-\Delta \mathrm{CT}}$ ) (data presented in this figure originates from figure 7B).

\section{Selection of good quality oocytes}

Following the insemination of all 227 MII oocytes, 96\% (24/25) of woman had at least one oocyte considered to be of good quality as measured by good blastocyst development and/or pregnancy.

\section{Random selection}

The random probability of selecting a good quality oocyte from selecting a single oocyte from each woman was $22.9 \pm 2.7 \%$ for the 25 women. The random probability of selecting at least one 
good quality oocyte from selecting three oocytes from each woman was $50.3 \pm 4.4 \%$ across the same group of women.

\section{The use of ranking analyses for oocyte selection}

The combination (sum) of the ranking of four genes (HAS2, FSHR, VCAN and PR) in individual CC masses provided the highest chance of selecting a single oocyte with good developmental potential (blastocyst and pregnancy) when the CC mass with the highest ranking was selected. In this analysis, there were six cases where the highest-ranking $\mathrm{CC}$ mass shared a similar rank to a second CC mass that was associated with a negative outcome, further selection was made between the equally ranked samples according to the VCAN rank. In any case in which the VCAN rank was also equal, then further selection was made according to the FSHR rank. This method resulted in a 52\% (13/25 women) success rate of picking a good quality oocyte by selecting a single COC (Table 7 and Figure 7D).

In the case of selecting three COC (highest three) by the ranking method above described (according to four genes), the efficiency of selecting at least one oocyte with good developmental potential was 76\% (19/25 women) (Figure 7D). However, the combination of the rankings for the two genes $H A S 2$ and FSHR, provided the highest efficiency $(80 \% ; 20 / 25)$ for selecting at least one good quality oocyte (Table 7 and Figure 7D). 
Table 7: Summary of the percentage ( $\mathrm{N}=25$ patients) success rate in selecting a single oocyte with good developmental potential using the ranking of various combinations of CC-derived expression levels of different candidates genes and estimated numbers of $\mathrm{CC}$. The first column represents the parameter that was used for ranking. The second column (1x COC) shows the success rate when selecting a single COC with the highest ranking value. The third column $\left(\right.$ Shared Rank ${ }^{1}$ ) shows the number of patients where at least two COC shared the same highest ranking. The fourth column (3x COC) represents the success rate when three COC are selected according to the three highest rankings. The fifth column (Shared Rank ${ }^{2}$ ) shows the number of patients where all good quality oocytes within the three highest ranking COC shared rankings with at least a fourth COC.

\begin{tabular}{lcccc}
\hline Ranking & 1x COC & $\begin{array}{c}\text { Shared } \\
\text { Rank }^{\mathbf{1}}\end{array}$ & $\mathbf{3 x}$ COC & $\begin{array}{c}\text { Shared } \\
\text { Rank }^{2}\end{array}$ \\
\hline HAS2 & $40 \%(10 / 25)$ & $4 / 10$ & $80 \%(20 / 25)$ & $4 / 20$ \\
FSHR & $56 \%(14 / 25)$ & $8 / 14$ & $84 \%(21 / 25)$ & $7 / 21$ \\
ALCAM & $28 \%(7 / 25)$ & $3 / 7$ & $60 \%(15 / 25)$ & $4 / 15$ \\
VCAN & $44 \%(11 / 25)$ & $4 / 11$ & $84 \%(21 / 25)$ & $4 / 21$ \\
NRP1 & $40 \%(10 / 25)$ & $5 / 10$ & $72 \%(18 / 25)$ & $4 / 18$ \\
PR & $40 \%(10 / 25)$ & $3 / 10$ & $68 \%(17 / 25)$ & $4 / 17$ \\
CC numbers & $32 \%(8 / 25)$ & $5 / 8$ & $68 \%(17 / 25)$ & $4 / 17$ \\
HAS $2+F S H R$ & $48 \%(12 / 25)$ & $5 / 12$ & $80 \%(20 / 25)$ & $0 / 20$ \\
HAS $2+F S H R+V C A N+P R$ & $56 \%(14 / 25)$ & $6 / 14$ & $76 \%(19 / 25)$ & $0 / 20$ \\
\hline
\end{tabular}


i)
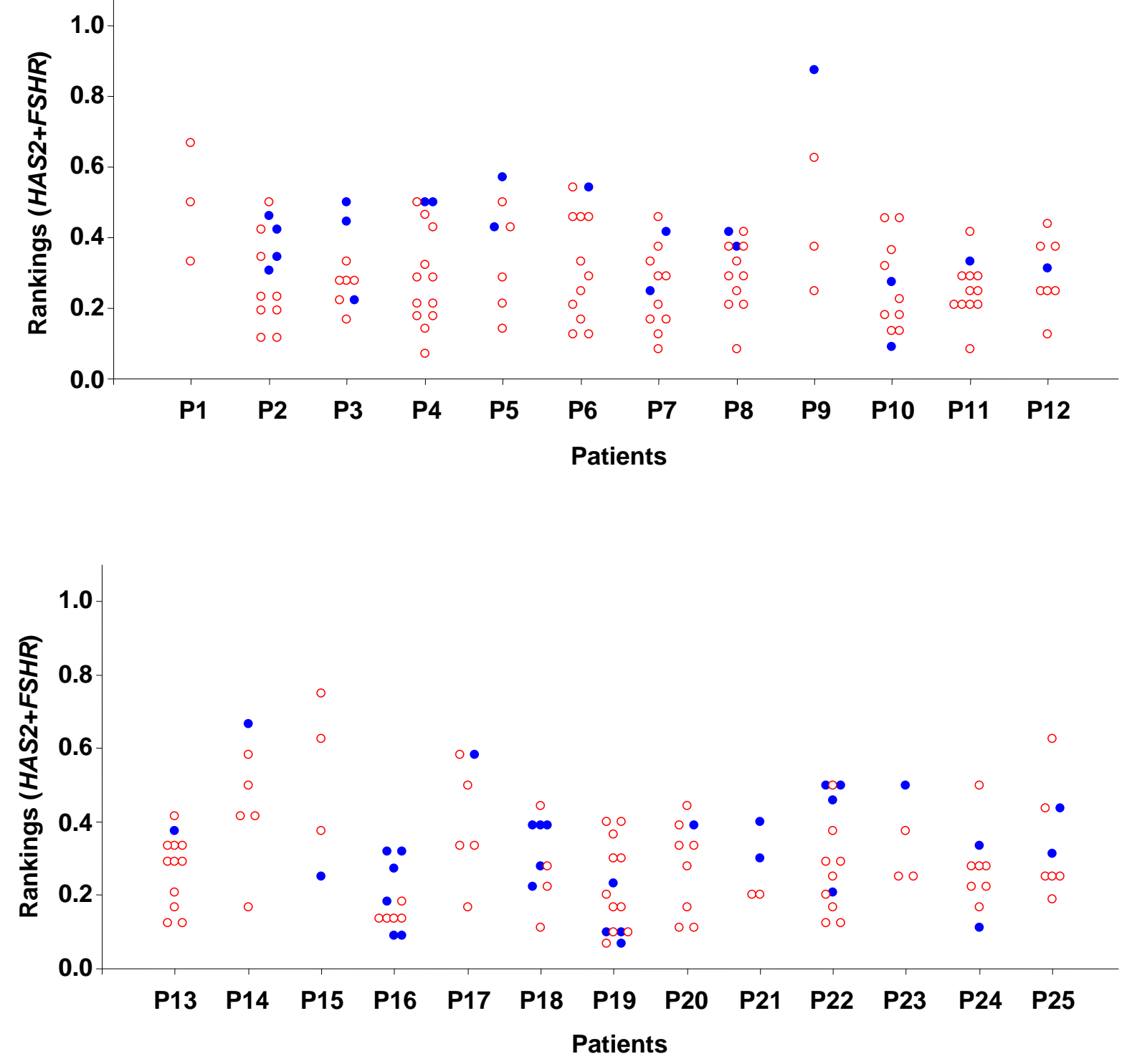

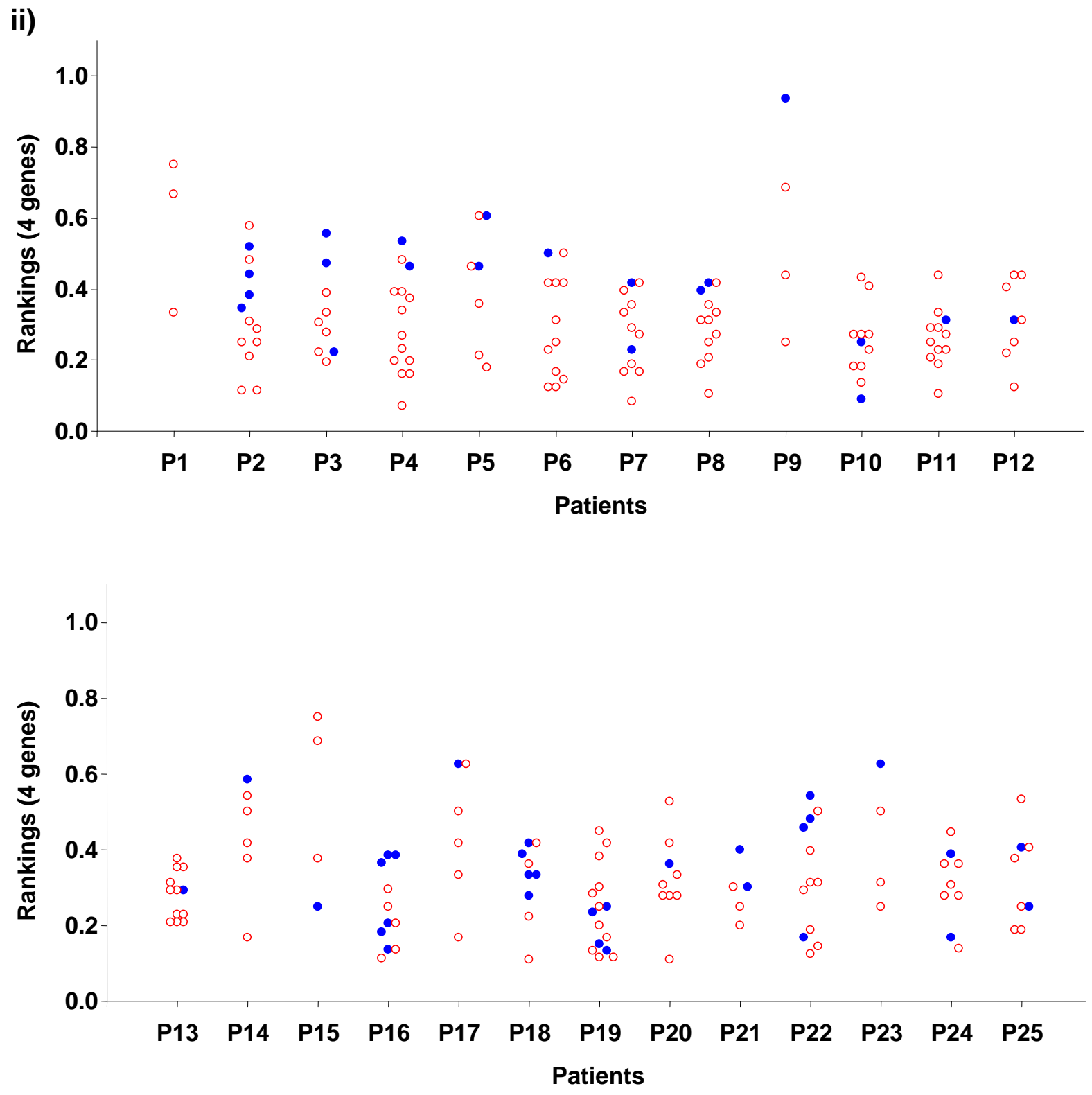

Figure 7D: The combined rankings of individual COC from 25 patients (P1-P25) according to mRNA levels of (i) HAS2 and FSHR or (ii) HAS2, FSHR, VCAN and PR. The blue circles represent those $\mathrm{COC}$ associated with oocytes that progressed to good quality blastocysts and/or pregnancy, whilst the red circles represent individual COC that were associated with oocytes with a negative outcome. The rankings are presented in proportions. To calculate the combined ranking value for individual $\mathrm{COC}$ within each patient 1) the rankings for individual COC for each gene was converted to proportions, by dividing the ranking number with the total number of COC 
within a patient and, 2) the ranking (in proportions) of each parameter was added and divided with the number of parameters taken in consideration for each individual COC.

\section{Oocyte selection efficiency}

The success rate $(96 \%)$ of recovering at least one good quality oocyte after follicle aspiration (i.e. when using all MII oocytes available) was significantly higher $(\mathrm{P}<0.0001)$ compared to that when a single oocyte was selected by the ranking method based on CC gene expression (52\%) or by random (23\%) and compared to that when three oocytes were randomly selected (50\%). However, when three oocytes were selected by the ranking method based on CC gene expression, the success rate of yielding at least one good quality embryo was similar that from using all 227 MII oocytes for injection (80\%; $\mathrm{P}=0.085)$. Selecting COC according to the ranking method provided a significantly higher chance of selecting at least one good quality oocyte compared to random selection (1x COC: $52 \%$ vs $23 \%, \mathrm{P}=0.008$; 3x COC: $80 \%$ vs 52\%, $\mathrm{P}=0.002$ ).

Oocyte selection based on the ranking method was further tested in women from Gp 2 and Gp 3 using the available HAS2 and FSHR expression data. However, the number of women recruited in Gp $2(\mathrm{~N}=11)$ and $\mathrm{Gp} 3(\mathrm{~N}=12)$ were significantly lower (less than half, $\mathrm{P}<0.0001)$ compared with those recruited in Gp $1(\mathrm{~N}=25)$. Furthermore in Gp 2 and Gp 3 the proportion of women with at least one good quality blastocyst available following treatment was further reduced [Gp 1: $63.6 \%$ (7/11), Gp 2: $25 \%$ (3/12)] compared to Gp 1 [96\% (24/25)]. For these reasons no statistical analyses were possible for the outcomes in Gp 2 and 3 women. Nevertheless, the efficiency of the ranking method was examined in Gp 2 but only in women who had at least one good quality blastocyst $(\mathrm{N}=7)$; the results were compared with those from Gp $1(\mathrm{~N}=24)$ women who had at least one good quality blastocyst available for treatment. In Gp 2 women (N=7), the selection of a single COC according to the ranking method provided a $28.9 \%$ chance of selecting an oocyte that resulted in a good quality blastocyst whereas random selection provided a $24.2 \%$ chance. The selection of three COC according to the ranking method provided an $85.7 \%$ chance of selecting an oocyte that resulted in a good quality blastocyst whereas random selection provided a $52.1 \%$ chance. The results (ranking compared to random: 1x COC: $28.9 \%$ vs $24.2 \%$, 3x COC: $85.7 \%$ vs $52.1 \%$ ) for the Gp 2 women while not reaching statistical significance with respect to the advantages in applying a ranking method of selection because of the lower 
numbers, they showed similar tendencies to those for the Gp 1 women ( $\mathrm{N}=24$, ranking compared to random: $1 \mathrm{x}$ COC: $54.2 \%$ vs $23.9 \%$, 3x COC $83.3 \%$ vs $52.4 \%$ ).

In $\mathrm{Gp} 3$ women there were too few data points and for this reason no meaningful results/conclusion were possible.

In the quest of identifying good quality oocytes further, a principal component analysis (PCA) was performed. However, no significantly different pattern of clustering was observed between oocytes associated with arrested development and those associated with good blastocyst development (Appendix 6).

\subsection{Discussion}

The most important finding of this study is that the measurement of candidate gene levels in CC and using a ranking analysis for individual COC provides a more reliable method for selecting good quality oocytes then by random selection. Moreover, using an mRNA-based ranking approach, it is possible to reduce the number of oocytes for ICSI to three. Not surprisingly, the most successful combinations of rankings for selecting good quality oocytes proved to be the combination of genes with the highest potential as molecular markers for blastocyst and pregnancy outcomes described in Chapter 6. The combination of the expression levels of four genes (HAS2, FSHR, VCAN and $P R$ ) provided the highest chance (52\%) for selecting a good quality oocyte when attempting to select only one oocyte for IVF. Although this method provided $76 \%$ efficiency for selecting at least one good quality oocyte when three oocytes were selected, the combination of the gene expression of two genes (HAS2 and FSHR) provided an even higher (80\%, $\mathrm{P}<0.05$ compared with selecting one oocyte using the ranking of four genes (52\%)) efficiency for the same analyses.

The selection potential of the ranking method using HAS2 and FSHR data was also tested in the much smaller groups of young women in Gp 2 and Gp 3 but not Gp 4 (due to all women having DET procedures). The differences in selection efficiency between ranking and random selections for Gp 2 showed similar trends to those in Gp 1 although the results did not reach statistical significance due to the lower number of women and COC available for analysis. These results 
however, are promising and show that the application of hierarchical ranking method may also be applicable to oocytes from young women with PCOS. For the Gp 3 women no meaningful conclusion can be drawn as there were too few data points: the good blastocyst rate was only $7.9 \%$ (3/38). For these reasons, the ranking method for oocyte selection described in this study would be recommended for investigation in future larger studies dealing with young women with PCOS and young women with diminished ovarian reserve.

As presented in Table 7, the selection of good quality oocytes using a single candidate gene approach can often reach very similar levels in comparison to selections based on multiple combinations. However, when using single genes, in the case of equally ranked COC, it is impossible to decide which COC should be selected. In contrast, when combinations of multiple genes were used, the number of COC sharing similar ranks was highly reduced (i.e. all patients who had good quality oocytes in the three highest ranking $\mathrm{COC}$, had at least one good quality oocyte that did not share similar rankings with a fourth $\mathrm{COC}$ within a patient), and even in the case of equally ranking COC (i.e. selecting the single highest ranking COC), differentiation between two COC could be made by adding more weight to one or two of the genes (i.e. VCAN and FSHR) selected in the prediction model. Further, we report that the ranking of individual $\mathrm{COC}$, according to $\mathrm{CC}$ numbers added no beneficial value to the selection efficiency, and again this highlighted the poor selection potential of morphological markers (Rienzi et al. 2011). The present study also reports that the principal component analysis conducted for the six genes in the 227 individual CC masses has not shown any clear differential clustering in regards to blastocyst development, and added no power to the selection of good quality oocytes. However, it is believed that PCA would likely be a very powerful analytical tool for examining large numbers of genes following DNA microarray or RNA-Seq as this could be used to select the most strongly associated genes for further ranking studies (Guo et al. 2010).

To the best of our knowledge, the present study is the first to investigate the uniformity of MII oocytes according to mRNA levels in associated $\mathrm{CC}$ and somatic cell composition of individual $\mathrm{COC}$ in relation to blastocyst development and pregnancy outcome. Most studies that investigate candidate mRNA levels in $\mathrm{CC}$ in relation to blastocyst development and pregnancy outcome report their findings as group means and, in the majority of cases, these outcomes are not relative 
to total number of MII oocytes retrieved (Feuerstein et al. 2007; Gebhardt et al. 2011; Wathlet et al. 2011). In this study, the results are reported for individual COC and relative to the total number of MII oocytes collected following follicle aspirations.

In stimulated IVF cycles, OCP and GnRH agonists are often administered to suppress gonadotrophin secretion and reduce the occurrence of large dominant follicles. This allows a cohort of small antral follicles to respond to exogenous rFSH treatment in a more co-coordinated manner, thereby enhancing the numbers of mature oocytes for recovery and facilitating the timing of egg collections (Gonen et al. 1990; Merton et al. 2003; Huirne et al. 2004; Huirne et al. 2006). However, despite a high degree of standardization and synchronization of the stimulated IVF cycles in the present study, the results from this paper demonstrate a high level of heterogeneity in $\mathrm{CC}$ cell numbers and gene expression levels notwithstanding the large number of oocytes progressing to MII. Following pre-treatment with OCP, down-regulation with GnRH agonist and stimulation with exogenous $\mathrm{rFSH}, 43 \%$ of pre-ovulatory follicles were of identical size on the day of administration of the ovulatory hCG. However, from the 227 MII oocytes collected, only two had similar CC numbers and shared similar mRNA levels in CC for all six candidate genes. Thus, it becomes evident now that the current ovarian gonadotrophin assisted stimulation regimens do not synchronize follicular development in humans (measured by mRNA levels and cell composition of individual COC). These results add further evidence to the findings that follicular recruitment and development is continuous and sequential (Peters et al. 1975), and that gonadotrophin assisted stimulation regimens do not override the hierarchical pattern of follicular development (first documented in sheep) and are unlikely to improve developmental synchrony of the follicles recruited (McNatty et al. 2010). 


\section{Chapter 8 DISCUSSION}

\subsection{Introduction}

The aim of every assisted reproduction treatment is to assist the birth of a single healthy infant (Land and Evers 2003). Although significant increases in pregnancy rates have been achieved (Gardner and Sakkas 2003), there are still substantial improvements which could be made. One such improvement would be the development of a reliable method for selecting oocytes with the best developmental potential to become a viable blastocyst and result in a positive pregnancy and a healthy baby (Rienzi et al. 2011).

In the present study, the main objective was to investigate the feasibility of CC markers for the selection of oocytes to develop into good quality blastocysts and/or positive pregnancy outcomes. To address this objective the relative expression levels of eight candidate genes and the CC compositions of individual COC were determined. These parameters then were compared with subsequent developmental indicators namely good blastocyst development on day 5/6 and pregnancy outcome (Day 14 serum $\mathrm{hCG} \geq 18 \mathrm{iu} / 1$ and live birth) after all MII oocytes were subjected to ICSI.

\subsection{Validation of a multiplex QPCR method}

The first major achievement of the present study was the validation of a multiplex QPCR method that allowed the simultaneous measurement of four genes (quadriplex QPCR) in CC of individual $\mathrm{COC}$ in a single reaction. To our knowledge, the present study is the first to investigate candidate mRNA levels in human CC from individual COC by a quadriplex TaqMan QPCR method (McKenzie et al. 2004; Feuerstein et al. 2007; Adriaenssens et al. 2010; Gebhardt et al. 2011; Wathlet et al. 2011). The validation and the use of the multiplex (triplex and quadriplex) QPCR methods in this paper have the advantage over singleplex QPCR reactions by increasing the efficiency by measuring the expression of multiple genes in a single reaction: this results in several advantages such as reducing the amount of time and labour, savings in reagents and maximizing the use of template required (Swango et al. 2007; Hudlow et al. 2008). In the present 
study, the measurements of eight candidate genes (HAS2, FSHR, SLC2A4 (or GLUT4), VCAN, $A L C A M, S F R P 2, P R$ and $N R P 1)$ ) are reported using approximately $7 \mu$ l (out of a total $20 \mu 1$ ) of available template. If all $20 \mu \mathrm{l}$ of the template was used, this would allow the measurement of at least 20 candidate genes in CC from individual COC.

The key aspects of the validation process of the present multiplex method were: 1) Verifying the quality of the $\mathrm{CC}$ samples by measuring the viability (86.4\%) of the $\mathrm{CC}$ after denudation, before freezing the samples $\left(-196^{\circ} \mathrm{C}\right)$ for mRNA extraction. This was a key issue considering the possible negative effects of enzyme treatment (hyaluronidase) and mechanical denudation (glass pipette) on viability of the CC. High cell viability was also important considering the well documented negative effects of cell apoptosis on DNA integrity that impact the appropriate quantification of genes in tissue studies (Koppelkamm et al. 2011). The aforementioned validation step had not previously been reported for expression studies on human CC (McKenzie et al. 2004; Feuerstein et al. 2007; Adriaenssens et al. 2010; Gebhardt et al. 2011; Wathlet et al. 2011). Another key validation step was that concerning the housekeeping gene, RPL19. Importantly, this gene was expressed in a similar range to many of the target genes in this study. The validation of the RPL19 gene was achieved by demonstrating a strong correlation between mRNA ( $\mathrm{C}_{\mathrm{T}}$ values) in $\mathrm{CC}$ and numbers of $\mathrm{CC}$ and also the mRNA levels of a widely recognized and highly abundant housekeeping gene, 18S. Given its abundance, $18 S$ was unsuitable for quantitation of genes in $\mathrm{CC}$ which were expressed at much lower levels.

A significant advantage in using the housekeeping gene (RPL19) was that it was possible to determine the $\mathrm{CC}$ numbers from individual COC (Chapter 3 and 4). Moreover, from using RPL19, it was determined that the minimal number of $\mathrm{CC}$ needed to accurately measure the expression levels of multiple genes by multiplex QPCR was $\sim 70$.

Whilst there are clear advantages of multiplex TaqMan QPCR over singleplex QPCR (SYBR Green or TaqMan) (Swango et al. 2007; Hudlow et al. 2008), the limitation of the multiplex TaqMan QPCR is that it only allows a maximum of four genes to be measured in a single reaction. Recently it was reported that the application of a GeXP genetic analytical system permits the quantitation of up to 35 genes simultaneously in a single reaction (Rai et al. 2009). 
Similarly to the TaqMan QPCR method, the GeXP system allows the analyses of target and housekeeping samples in the same experiment. A further advantage of this method is that the entire procedure, after RNA isolation can be completed in six hours (Rai et al. 2009). For these reasons, for future studies it is suggested that the application of the GeXP genetic analytical system would significantly increase the chances of identifying many more informative candidate genes in CC more rapidly.

\subsection{CC numbers in individual COC}

The validation of the housekeeping gene, RPL19, also permitted an investigation of the relationship between $\mathrm{CC}$ numbers and oocyte developmental indicators. Based on an analysis of 608 individual $\mathrm{CC}$ samples, the mean number of $\mathrm{CC}$ present in an individual COC recovered before IVF treatment was 28872; this number is much higher than 11500 which had been reported previously in a much smaller study (Feuerstein et al. 2007). Correlation analyses (see Chapter 4) showed that CC numbers in individual COC were found not to be related to good blastocyst development and/or pregnancy adding further evidence to the hypothesis, that morphological characteristics of individual COC are poor markers of oocyte quality ( $\mathrm{Ng}$ et al. 1999; Rattanachaiyanont et al. 1999; Lee et al. 2001; Moffatt et al. 2002; Corn et al. 2005).

\subsection{CC mRNA levels in individual COC}

In the present study, the mRNA levels of eight candidate genes were assessed in CC. Six of these genes (HAS2, FSHR, ALCAM, VCAN, NRP1 and PR) were detectable in $98.5 \%$ of the samples, whereas the expression levels of GLUT4 were low and those for SFRP2 were undetectable in individual CC masses. These findings are in agreement with previous reports, showing that most CC expressed genes can be quantified in single COC (McKenzie et al. 2004; Hasegawa et al. 2005; Adriaenssens et al. 2010; Assidi et al. 2011; Wathlet et al. 2011). Furthermore, using linear correlation analyses it was found that the mRNA levels $\left(\mathrm{C}_{\mathrm{T}}\right.$ values $)$ of the six abundant genes in individual CC masses were significantly correlated, where the relative expression levels of VCAN mRNA were the highest, followed by those of NRP1, HAS2, ALCAM, FSHR and PR. It has to be 
acknowledged however, that comparing relative expression levels ( $\mathrm{C}_{\mathrm{T}}$ values) by QPCR methods has its limitations, for instance it is known that the type of dye used, and the composition of the sequence can influence the sensitivity of the QPCR measurement (Gudnason et al. 2007)

CC mRNA levels in CC are affected by the endocrine status of the individual as well as by the exogenous rFSH treatment

In the process of identifying reliable oocyte quality markers, it was important to investigate the possible effects of biological and treatment variables on mRNA levels in the CC. For this study three candidate genes were selected (HAS2, FSHR and GLUT4) and were investigated in women $(\mathrm{N}=68)$ enrolled in the four study groups (young and healthy women, young women with PCOS, young women with diminished ovarian reserve and, older and healthy women).

The expression levels of these genes were investigated in relation to the infertility background of the women that had been recruited for these studies. It was found that the expression levels of FSHR in CC are significantly up-regulated in young women with PCOS compared with young and healthy women and young women with diminished ovarian reserve. This finding correlates well with other reports studying the expression of FSHR in pooled GC samples from women with PCOS, normal ovarian function and with poor ovarian reserve (Almahbobi et al. 1996; Cai et al. 2007; Catteau-Jonard et al. 2008; Gonzalez-Fernandez et al. 2010). Further, it was found that the expression levels of HAS2 were elevated in older women and in young women with diminished ovarian reserve, and were the lowest in women with PCOS. The measurement of mRNA levels of GLUT4 added little to these analyses, due to the low levels of expression in individual CC masses.

The expression levels of $H A S 2$ and FSHR in individual CC masses were also examined in relation to certain biological and treatment variables. It was found that FSHR mRNA levels were not correlated to any of the biological or treatment variables. On the other hand, the mRNA levels of HAS2 were correlated with age (a finding previously described by Adriaenssens et al. (2010)), basal serum FSH and AMH levels. However, HAS2 mRNA showed the strongest correlation with the amount of exogenous $\mathrm{rFSH}$ used for stimulation, and to the ovarian response (follicle numbers and COC collected). Taking into account the role of FSH in regards to hyaluronic acid 
synthesis, it is reasonable to assume that the HAS2 mRNA levels are primarily correlated to serum FSH levels, whilst the influence of age is, at best, a secondary factor. This is further supported by the finding that HAS2 mRNA levels in $\mathrm{CC}$ of young women with diminished ovarian reserve and older women were similar.

These investigations highlight the heterogeneity of $\mathrm{CC}$ expressed genes in relation to background characteristics. The expression of some genes (i.e. FSHR), are affected by the endocrine status of the individual, whilst others (i.e. HAS2), are affected primarily by the exogenous rFSH treatment.

\section{CC mRNA levels are predictive of oocyte maturity}

The present paper adds further evidence to the findings that HAS2 and VCAN are good predictors of oocyte maturity (McKenzie et al. 2004; Adriaenssens et al. 2010; Wathlet et al. 2011). Both of these genes were significantly up-regulated in $\mathrm{CC}$ associated with immature oocytes (GV and MI) compared with CC with MII oocytes. Studies in mice COC report that the expression of these genes is rapidly initiated after exposure to ovulatory hCG and then gradually decrease during and after ovulation (Adriaenssens et al. 2011). However, as evidenced in the present study, the expression of these genes is higher in $\mathrm{CC}$ associated with good quality oocytes compared with CC from oocytes with negative outcomes (Chapter 6). It is reasonable to believe that during ovulation, poor quality oocytes fail to maintain appropriate expression levels of these genes in CC which, in turn, leads to a low level of mucification of the CC mass.

\section{CC mRNA levels are predictive of blastocyst development and live birth outcome}

A significant finding reported in this study is that the mean mRNA levels of HAS2, VCAN and $P R$ are significantly increased in $\mathrm{CC}$ of oocytes associated with embryos that progressed to pregnancy and/or good quality blastocysts compared to the expression levels in CC associated with oocytes with failed development (Chapter 6). Furthermore, it was found that the mean mRNA levels of VCAN in CC of oocytes associated with live birth were significantly higher compared to those in $\mathrm{CC}$ associated with oocytes with failed development. A highly important aspect of these findings is that these results are relative to the total number of MII oocytes available after follicular aspiration. Previous studies that showed elevated expression levels of candidate genes in $\mathrm{CC}$ associated with blastocyst development and/or pregnancy outcome 
reported most of their findings for selected groups (i.e. positive and negative groups of embryos selected for transfer) and not relative to total MII oocytes retrieved (Feuerstein et al. 2007; Gebhardt et al. 2011; Wathlet et al. 2011). Whilst, the findings of the latter studies are informative, it would be of much greater value to identify good quality oocytes within the total number of MII oocytes available.

The present study further confirmed that blastocyst development and live birth outcome are the critical stages against which the value CC gene expression should be assessed (Gebhardt et al. 2011; Wathlet et al. 2011). Any earlier assessment of oocyte quality leads to variable and inconsistent results. However, for a better understanding in the $\mathrm{CC}$ gene functions it is proposed that future studies should report data on every possible embryological assessment from Day 0 to Day 6; these data would greatly assist in reducing the variability and enabling better comparisons to be made between published reports.

\section{Candidate genes recommended for future investigations}

As highlighted in the above section and in Chapter 6 and 7, the most informative genes used in this research experiment were $H A S 2, F S H R, V C A N$ and $P R$ and these are suggested for inclusion with other potential candidates in future investigations.

To our knowledge, the present study is the first to investigate hyaluronan synthase 2 (HAS2) mRNA levels in relation to blastocyst development and pregnancy outcome relative to total MII oocytes collected following follicular aspirations. HAS2 is one of the key proteins involved in the formation of the extracellular matrix and the expansion of the CC mass (Fulop et al. 1997; Salustri et al. 1999). Furthermore, HAS2 mRNA expression is induced by the ovulatory LH surge and it is regulated by OSF in the presence of FSH (Gui and Joyce 2005).

The present study examined the relationship between follicle stimulating hormone receptor $(F S H R)$ mRNA levels and oocyte developmental indicators. Although, the mean expression level of FSHR showed only a tendency towards higher levels in CC associated with good quality oocytes, the mRNA levels of individual COC provided new information when the levels in CC associated with MII oocytes within individual women were subjected to ranking analysis 
(Chapter 7). The finding that FSHR was one of the more informative genes in this analysis is not surprising considering that the acquisition of FSHR in CC is vital for the action of FSH and that this acquisition is regulated by OSF (Otsuka et al. 2005). Furthermore in a non-stimulated monoovulatory animal model (bovine) it was reported that FSHR mRNA levels in CC increased with follicle diameter (Caixeta et al. 2009) highlighting the critical role that FSHR have in the development of the dominant follicle.

Versican $(V C A N)$ is a hyaluronan binding factor involved in the formation of the extracellular matrix of the COC (Russell et al. 2003). In this research, the expression levels of VCAN were significantly higher in CC associated with oocytes that developed to blastocyst and pregnancy. More importantly, the mRNA levels of $V C A N$ were higher in $\mathrm{CC}$ associated with oocytes that resulted in live births. Previously, the expression levels of VCAN showed conflicting results, in one study they showed lower expression levels in CC associated with oocytes that progressed to blastocyst, but higher levels in $\mathrm{CC}$ associated with oocytes that progressed to pregnancy (Gebhardt et al. 2011; Wathlet et al. 2011). However in these reports the expression levels of VCAN in regards to blastocyst development were not investigated relative to total number of MII oocytes submitted for fertilization (Wathlet et al. 2011). Similarly, in relation to pregnancy, expression levels of VCAN were only investigated in embryos transferred and again not relative to total number of oocytes retrieved (Gebhardt et al. 2011; Wathlet et al. 2011). The findings presented in this research are related to all MII oocytes available for each woman, again highlight the importance of analysing the data in relation to total oocytes collected.

The finding that progesterone receptor $(P R)$ levels were significantly higher in CC associated with good quality oocytes is perhaps not surprising considering the well documented increase in PR expression levels in GC of pre-ovulatory large follicles following the pituitary LH surge (species: rat and mice) (Park and Mayo 1991; Kim et al. 2008; Robker et al. 2009). However, in the single study that investigated the $\mathrm{PR}$ expression levels in $\mathrm{CC}$ in relation to embryo development, the author's results were inconclusive (Hasegawa et al. 2005). However, it is important to note that these results were reported for early embryo cleavage. Therefore, this emphasizes once more that the critical stages for which CC gene expression should be compared to, are blastocyst development and pregnancy outcome. 
Based on evidence of previous reports (Gebhardt et al. 2011; Wathlet et al. 2011) and results presented in this study, for future interrogations it is recommended that these include other genes involved in the formation of the extracellular matrix of the COC, such as prostaglandinendoperoxide synthase 2 (PTGS2). Indeed the expression levels of PTGS2 were significantly higher in $\mathrm{CC}$ of oocytes that progressed to pregnancy/live birth compared to negative outcomes amongst oocytes that resulted in a transfer (Gebhardt et al. 2011; Wathlet et al. 2011). A further candidate gene recommended is syndecan 4 (SDC4), an anticoagulant heparan sulphate proteoglycan, that is synthesized in granulosa cells under the influence of gonadotrophins (Princivalle et al. 2001). Moreover, higher expression levels of SDC4 were reported in CC associated with positive outcomes (good blastocyst or pregnancy) compared to negative outcomes in human IVF cycles (Wathlet et al. 2011). The mRNA levels of these two genes in CC associated with blastocyst development and live birth outcome relative to all MII oocytes retrieved however still remains to be investigated.

\section{Candidate genes with poor potential for oocyte selection}

The present study confirmed when all MII oocytes retrieved are considered in the analysis the mRNA levels of GLUT4, ALCAM, SFRP2 and NRP1 in CC are poor predicators for oocyte development. Unlike in previous reports where it was reported that the mean expression levels of ALCAM (Adriaenssens et al. 2010; Wathlet et al. 2011) and NRP1 (Assidi et al. 2011) were higher in positive groups compared to negative groups, the present study found that the mean expression level of these genes are not indicative of good blastocyst development and/or pregnancy outcome. Whilst GLUT4 and SFRP2 are abundant genes in mice CC during the final steps of oocyte maturation (Roberts et al. 2004) and ovulation (Hernandez-Gonzalez et al. 2006), these genes were lowly expressed in individual COC in humans in the present study and thus cannot be investigated in regards to embryo development. 


\subsection{The ranking of individual MII oocytes according to CC mRNA levels, and the application of this ranking system for oocyte selection}

The most important finding of the present paper is that it demonstrated that the selection of good quality oocytes in stimulated IVF cycles is possible. This finding is based on the results of the ranking method (see Chapter 7) which showed that: 1) good quality oocytes (at least one) can be selected with an $80 \%$ efficiency, whilst reducing the number of oocytes required for insemination to no more than three (in the case of this study from 9.1 to 3 (67\% reduction)) and; 2) further evidenced that exogenous FSH treatment does not override the hierarchical pattern of follicular development (McNatty et al. 2010).

Based on present evidence, the ranking method described in this study is superior to any current morphological criteria for oocyte selection ( $\mathrm{Ng}$ et al. 1999; Rienzi et al. 2003; Rienzi et al. 2011), morphological evaluation of the COC (Ng et al. 1999; Rattanachaiyanont et al. 1999; Lee et al. 2001; Moffatt et al. 2002) or metabolomic profiling of the oocyte culture media (NelThemaat and Nagy 2011). Moreover this system offers a similar chance of identifying good quality oocytes (at least one) compared to classical treatments where all MII oocytes are used for fertilization.

As evidenced in Chapter 6, analysing mean mRNA levels in $\mathrm{CC}$ is a suitable method for identifying good candidate genes for oocyte selection. However, as presented in Chapter 7, for a successful implementation of an oocytes selection method, mRNA levels in CC must be interrogated for individual COC. Not surprisingly, the highest success rates for the selection of good quality oocytes (at least one) resulted following the various combinations of rankings of the four genes (HAS2, VCAN, $P R$ and FSHR) that showed higher mean mRNA levels for good blastocyst development and/or pregnancy across all samples.

In the ranking analyses, two scenarios were investigated: a) the selection of a single oocyte and, b) the selection of three oocytes. These numbers were chosen keeping in mind current ethical, legal, social and religious requirements for oocyte selection (Kufner et al. 2009; Mohler-Kuo et al. 2009; Levi Setti et al. 2011). Whilst the selection of a single oocyte according to the ranking 
method was significantly more successful than random selection, the selection of three oocytes by the ranking method provided the best chance of selecting good quality oocytes. Not only was this method significantly better than the random selection of three oocytes, it also provided a similar chance of selecting good quality oocytes compared to using all MII oocytes available after follicle aspiration. When all MII oocytes are used for treatment, as many as six good quality embryos may be produced with one to be transferred and five to be frozen, (see Chapter 7), showcasing the high probability of producing surplus embryos. The insemination of only three oocytes doesn't necessarily mean that the potential of those not inseminated have to be discarded as surplus oocytes can be cryopreserved, in the case that further siblings are required later (Ubaldi et al. 2010). Furthermore this method could be used to overcome the ethical and legal challenges associated with cryopreservation of surplus embryos which is common in treatments where all available oocytes are used for insemination (Fabbri et al. 1998; Bankowski et al. 2005).

An equally important aspect of the mRNA based ranking method is the high pregnancy rates achieved in young and healthy women. When all MII oocytes collected were taken in consideration a positive hCG pregnancy of $84 \%(21 / 25)$ and a live birth in $76 \%(19 / 25)$ of women was achieved following fresh SET. When the highest three ranked MII oocytes were selected using the sum of ranks for HAS2 and FSHR mRNA levels, a pregnancy rate of $60 \%$ $(15 / 25)$ and a live birth rate of $52 \%(13 / 25)$ was calculated. This rate was achieved by adding the cases where the three highest ranking oocytes included the oocyte that was selected for transfer and resulted in a live birth, with the cases where although the three highest ranking oocytes did not include the oocyte that progressed to live birth, included at least one good quality blastocyst that could have been selected for transfer. To calculate the potential success rate of these blastocyst Fertility Associates (Wellington clinic) fresh and frozen-thawed blastocyst SET pregnancy (fresh: 67\%, frozen: 43\%) and live birth (fresh: 42\%, frozen: 29\%) results were considered (year 2009-2011, unpublished data). These results (pregnancy rate of 60\% and live birth rate of 52\%) although seem lower, are similar (hCG pregnancy: $\mathrm{P}=0.061$, live birth: $\mathrm{P}=0.080$ ) to those achieved by using all MII oocytes for insemination. Nevertheless the success rates achieved by the ranking selection method are more than comparable with international standards that use all MII oocytes for inseminations (Gardner et al. 1998; Abdalla 2010; Levi Setti et al. 2011; Zander-Fox et al. 2011). 
The limitations of the ranking method presented in this paper lie in: 1) its relatively lengthy process (mRNA extraction $\sim 3$ hours, reverse-transcription $\sim 2$ hours, TaqMan QPCR method 2-4 hours, and the time for operator manipulations); and 2) it has only been validated for young and healthy women, thus there is no information on how biological (i.e. PCOS) and treatment variables impact on rankings Until these questions are answered, this selection method has the limitations that it can only be used in oocyte freeze cycles or to enhance embryo selection in fresh IVF cycles in young and healthy women. However, with the use of the GeXP genetic analytical system, these limitations associated to $\mathrm{CC}$ markers could be addressed and minimized. By further automation of the GeXP method, the time required for mRNA measurement could be shortened, and up to 35 genes could be simultaneously investigated (Rai et al. 2009) and thus possibly further increasing the efficiency of oocyte selection.

\subsection{Investigations regarding the developmental synchrony of individual COC based on CC mRNA levels and CC numbers}

After analysing individual MII oocytes by the ranking method it became evident that, although $43 \%$ of the pre-ovulatory follicles were identical in diameter on the day of ovulation trigger, only two of the $227 \mathrm{COC}(0.3 \%)$ shared an identical CC number and mRNA levels for all six genes (HAS2, FSHR, ALCAM, VCAN, NRP1 and PR). The level of heterogeneity for individual COC associated with MII oocytes for individual parameters were 55\% (HAS2), 55\% (FSHR), 69\% (ALCAM), 61\% (VCAN), 56\% (NRP1), 62\% (PR) and, 61\% for CC number).

These findings for CC parameters are in line with those reported by McNatty (McNatty 1978; McNatty and Baird 1978) in humans and McNatty et al. (2010) in sheep. These authors showed that few if any antral follicles share identical endocrine microenvironment (steroid and gonadotrophin concentrations in the follicular fluid) and somatic cell composition (granulosa cells) even when they are of equal size (McNatty 1978c; McNatty et al. 1981b; McNatty et al. 1984). Furthermore, evidence in mono-ovulatory animal models (sheep) show that there is a considerable heterogeneity with respect to responsiveness to gonadotrophins in individual follicles within an individual (McNatty et al. 2010). Taking in consideration the aforementioned 
findings, the very low birth rates $(\sim 7 \%)$ from oocytes collected in stimulated IVF cycles (Li et al. 2008; Patrizio and Sakkas 2009) and the results from the present study, it can be concluded superovulation regimens do not improve developmental synchrony of the follicles recruited/oocytes collected. In other words, the exogenous rFSH treatment does not override the hierarchical pattern of follicular development. The higher success rates obtained in stimulated IVF cycles compared to natural IVF cycles (Fishel et al. 1985; Pelinck et al. 2002) are likely attributed to the higher number of follicles recruited and triggered at any moment in time. These findings on $\mathrm{CC}$ gene expression raise the possibility that even when several oocytes go on to form blastocysts, the gene expression profiles in these embryos may be rather different. Moreover these findings confirm what is already acknowledged that current hormonal stimulation protocols are inefficient and that alternative follicular maturation methods are required to enhance the yields of viable oocytes.

\subsection{Clinical implications of the findings presented in this study}

The findings of the present paper may have important implications for the future management of IVF procedures. The ranking method presented in this thesis for the selection of good quality oocytes, could provide a starting point to address the ever-increasing demand for oocyte selection. This demand is driven by several ethical, legal, financial and health factors. The ethical demands are highlighted by the increasing numbers of surplus embryos following superstimulated IVF cycles (Bankowski et al. 2005). There are religious considerations especially in the case of those believers who place high moral values on the status of the embryo and for whom embryo preservation is not an option (Schotsmans 1998; Mohler-Kuo et al. 2009). In some countries (i.e. Germany, Switzerland and Italy) there are legal restrictions on the number of embryos (in most cases to three embryos) that can be generated in any one treatment cycle (Kufner et al. 2009; Mohler-Kuo et al. 2009; Levi Setti et al. 2011) and this can sometimes compromise pregnancy rates (Levi Setti et al. 2011). Oocyte selections paired with early stage (i.e. Day 1 or Day 2) embryo replacements could also minimize the possible negative effects of extended in vitro culture of embryos on the health of the children conceived (Nelissen et al. 2012). There are financial benefits from an improved oocyte selection method through increased efficiencies with management and decreased financial costs of treatment (see Chapter 1). There 
are also benefits in cases where women undergoing oocyte cryopreservation could be more appropriately advised of the quality and number of oocytes needed to be frozen in order to achieve a live birth. Finally, there are also benefits with respect to assisting SET procedures and minimizing the prevalence of multiple gestations (Bissonnette et al. 2007).

\subsection{Conclusions}

The main objective of the present thesis was to assess the feasibility of CC markers for the selection of good quality oocytes in super-stimulated IVF treatments in humans. From these investigations, three major milestones were achieved. Firstly, a multiplex QPCR technique was validated, that was capable of measuring simultaneously the mRNA levels of four genes in CC and permitting an accurate measure of $\mathrm{CC}$ numbers in individual COC. Secondly, the ranking method based on the three multiplex QPCR reactions described herein permitted the selection of good quality oocytes (at least one) with $80 \%$ efficiency, whilst reducing the number of oocytes required for insemination by $67 \%$ (to no more than three). Moreover, the efficiency of selecting good quality oocytes (at least one) with a live birth outcome achieved by the ranking method provided similar rates to fresh IVF treatments using all MII oocytes for insemination. Thirdly, the finding that $99.7 \%$ of individual COC were significantly different in CC mRNA levels and in cell composition added further evidence to the findings that super-ovulation regimens do not improve developmental synchrony of the follicles recruited and do not override the hierarchical pattern of follicular development (McNatty et al. 2010). 


\section{APPENDICES}

The protocols presented in appendices 2.1, 2.2, 3.1, 3.2 and 3.3 are the intellectual property of Fertility Associates (FA) Ltd. These protocols are published according to FA's formatting policies. Permission for the publication of these protocols in the present thesis was granted by Dr. John Peek, Group Operation Manager, Fertility Associates Ltd.

\section{APPENDIX 1}

\section{Patient Consent Form}

\section{Research about Proteins Secreted by the Egg into the Surrounding Cumulus Cells}

We,

and have been approached by the staff of Fertility Associates when we were advised of our ovulation "trigger" about a research study on cumulus cells.

We have been provided with the information sheet entitled "Research about Proteins secreted by the Egg in to the Surrounding Cumulus Cells" dated November 2008 which we have read, and understand and accept.

We have had all our questions since we were approached about the research satisfactorily addressed by our scientist (name). today.

We agree for cumulus cells that are removed from each egg prior to fertilization to be frozen and stored for subsequent analysis of proteins secreted by the egg rather than discarded. The samples will be stored using only our clinic number ID.

Woman's signature:

Partner's signature

Date 


\section{APPENDIX 2.1}

Title Long course stimulation with pill for IVF, protocol 5.85

Author Dr. John Peek, Fertility Associates Ltd.

Purpose Routine stimulation for IVF/ICSI treatment, enabling adjusting the week of egg collection to smooth clinic workload

Responsibility Patient's doctor, doctor on duty for the day, authorised nursing and laboratory staff

Definitions Items in bold italics are mandatory, other items are guidelines

\section{SUPPRESSION}

- Microgynon 30 preferably starting on $1^{\text {st }}$ day of period, but at least within 4 days of period

- 400 ug Buserelin sc / day, starting 14-28 days after starting Microgynon

- Stop Microgynon 5-7 days after starting Buserelin

\section{DOWN-REGULATION CHECK}

E2 to check down-regulated after >= 14 days Buserelin, usually on Monday or Tuesday:

- If $\mathrm{E} 2<200 \mathrm{pM}$, then down-regulated.

- If E2 200-300 pM, then check hCG. If not pregnant, do scan.

Consider down-regulated if all follicles $=<5 \mathrm{~mm}$ AND endometrial thickness $=<3 \mathrm{~mm}$

- If $\mathrm{E} 2>300 \mathrm{pM}$ then check hCG. If not pregnant, do scan. If follicle(s) $>=16 \mathrm{~mm}$ then trigger with 2500 iu hCG. E2 to recheck for down-regulation in 7 days (whether hCG trigger on not).

\section{STARTING GONAL F / PUREGON}

- For starting dose, see 'Guidelines for dose of Gonal F/Puregon in IVF/ICSI'

- Dose of Gonal F/Puregon must be signed off on the management plan by a doctor

- Start Gonal F / Puregon once patient down-regulated 
- Reduce Buserelin to $200 \mathrm{ug} / \mathrm{d}$

- Adjust starting day of Gonal F/Puregon according to the starting dose:

$$
\begin{array}{lll}
\text { Starting dose } & <150 \mathrm{iu} / \text { day } & \text { Thursday } \\
& 150-300 \mathrm{iu} / \text { day } & \text { Wednesday }
\end{array}
$$

\section{MONITORING AND CHANGING DOSE}

- Any changes in dose must be checked and signed by a doctor within 48 hours

First step:

- E2 Tuesday (= day 7 Gonal F/Puregon for average responder)

- If E2 $<300 \mathrm{pM}$ then increase Gonal F/Puregon dose by 75 IU, plan E2 Friday, E2 \& scan Monday

- If E2 300-3000 pM then E2, scan Thursday, Friday OR Saturday (depending on E2 level and convenience)

○ If $\mathrm{E} 2>=3000 \mathrm{pM}$ then scan next day and decide whether to continue

Further steps:

- E2 and scan every 2-3 days until triggered with hCG

- If E2 rise between day $\sim 7$ and day $\sim 10>=1$ day behind ideal line, then increase Gonal F/Puregon dose providing E2 on day $7>=500 \mathrm{pM}$ and not 1 or 2 dominant follicles

- E2 day of trigger

\section{STOPPING}

- Decision to stop or coast must be made by a doctor or confirmed with a doctor

- If E2 remains low, OR follicles do not grow at expected rate $(2-3 \mathrm{~mm} / \mathrm{day})$, then recommend stopping

- If $<3$ preovulatory follicles, then discuss whether to continue or stop with patient

- If projected E2 day of hCG would be > 12,000 pM, then discuss coasting with doctor

- If abdominal discomfort, then doctor to discuss stopping with patient 


\section{TRIGGER}

- Trigger ovulation with 250 ug Ovidrel and stop Buserelin, when:

○ 2 follicles $>=20 \mathrm{~mm}$, or 3 follicles $>=18 \mathrm{~mm}, \mathrm{OR}$

○ Coasted and E2 falls to $<10,000 \mathrm{pM}$

- If wanting to change trigger day to smooth workload, trigger day earlier rather than later where possible.

- Do not give hCG trigger if $\mathrm{E} 2>20,000 \mathrm{pM}$

- If the E2 rise is significantly steeper than the standard line on the tracking sheet in the days leading to trigger, then a doctor must make the decision whether or not to trigger.

- If E2 low for expected number of follicles, then consider repeating scan.

OPU (= luteal day 0$)$

- $36 \mathrm{~h}$ after hCG trigger

- Premedication of 2 Panadol tablets (500 mg each).

- Other medication requires doctor's prescription.

\section{EMBRYO REPLACEMENT}

- At least $45 \mathrm{~h}$ after insemination in IVF, $43 \mathrm{~h}$ after injection in ICSI insemination

- If E2 > 12,000 pM day of hCG trigger or >= 20 eggs at $\mathrm{OPU}$, then freeze all embryos

- If 15-20 eggs at $\mathrm{OPU}$, then doctor's discretion whether to freeze all embryos

\section{LUTEAL SUPPORT AND MONITORING}

- Utrogestan intravaginal $2 \times 100 \mathrm{mg} 3 x$ / day from luteal day 1

- HCG test luteal day14

- If hCG >= 5 iu/l day 14, then consider pregnant, and do E2, P4, hCG day 18

- HCG result day 18 :

O If P4 > $80 \mathrm{nM}$, reduce to $100 \mathrm{mg}$ Utrogestan $3 \mathrm{x} /$ day for 4 days, then stop.

○ If P4 $<80 \mathrm{nM}$, stay on Utrogestan until scan with foetal heart at 10 weeks, then reduce to $100 \mathrm{mg}$ Utrogestan $3 x$ / day for 4 days then stop.

- Further monitoring at patient's request as early pregnancy monitoring service OR continue monitoring if any concern about possible ectopic pregnancy 


\section{DRUG SUMMARY}

Day 1 (to 4)

Day 14-28

After 7 days Buserelin

After $>=14$ days Buserelin

Next Thursday (+/- 1 day)

After 7 days Gonal F dominant follicle

Every 2-3 days

Follicles $>=18-20 \mathrm{~mm}$
Start Microgynon 30

Start Buserelin

Stop Microgynon

E2 to check down regulation

Start Gonal F/Puregon

E2 to check response, increase dose if no response, no

E2, scan to decide when to trigger

Trigger 


\section{APPENDIX 2.2}

Title Microdose flare stimulation for IVF, protocol 5.9

Author Dr. John Peek, Fertility Associates Ltd.

Purpose Stimulation for IVF/ICSI treatment when poor response expected

Responsibility Patient's doctor, doctor on duty for the day, authorised nursing and laboratory staff

Definition Items in italics are mandatory, other items are guidelines

\section{ORAL CONTRACEPTIVE PILL}

- Start Microgynon 30, preferably on $1^{\text {st }}$ day of period, but at least within 4 days of period, and continue for at least 21 day, or shorter duration between 14 and 21 with a doctor's approval.

\section{STARTING STIMULATION}

- Dose of Gonal F/Puregon must be signed off on the management plan by a doctor

- Start 50 ug Buserelin sc twice daily (bid) third day after stopping Microgynon

OR $0.015 \mathrm{mg}$ (0.15 ml) Decapeptyl sc twice daily

- Start Gonal F / Puregon two days after starting Buserelin

- For starting dose, see 'Guidelines for dose of Gonal F/Puregon in IVF/ICSI'

- Adjust starting days according the starting dose of Gonal F/Puregon:

$\begin{array}{lll} & \underline{225 \mathrm{IU} / \text { day }} & \underline{\text { 300 IU / day }} \\ \text { Last day Microgynon pill } & \text { Sunday } & \text { Friday } \\ \text { Start Buserelin (= day 1 stimulation) } & \text { Wednesday } & \text { Monday } \\ \text { Start Gonal F/Puregon } & \text { Friday } & \text { Wednesday } \\ \text { First E2 test } & \text { Monday (day 6) } & \text { Monday (day 8) }\end{array}$

\section{MONITORING AND CHANGING DOSE}

- Any changes in dose must be checked and signed by a doctor within 48 hours 
- Normally dose not changed for poor response (expect OPU $~ 2$ days later than initially expected)

First step:

- $E 2$ on $6^{\text {th }}-8^{\text {th }}$ day of stimulation

- If $\mathrm{E} 2<300 \mathrm{pM}$ then discuss with doctor

○ If E2 300-3000 pM then E2, scan 1-3 days later depending on E2 level

○ If $\mathrm{E} 2>=3000 \mathrm{pM}$ then scan next day

Further steps:

- E2 and scan every 2-3 days until triggered with hCG

- If leading 2-3 follicles on a Friday scan $>=15 \mathrm{~mm}$ and no trigger decision made, then rescan on Sunday, not Monday

$\circ$ If leading 2-3 follicles on a Friday scan $=<14 \mathrm{~mm}$ and trigger decision not yet made, then E2 and scan on Monday

- If E2 rise between day $\sim 6$ and day $\sim 9>=1$ day behind ideal line, then increase Gonal F/Puregon dose providing E2 on day $7>=500 \mathrm{pM}$ and not 1 or 2 dominant follicles

- Always do E2 day of trigger when expected E2 >=10,000 pM, or > 20 follicles on scan

\section{STOPPING}

- Decision to stop or coast must be made by a doctor

- If E2 remains low, OR follicles do not grow at expected rate $(2-3 \mathrm{~mm} / \mathrm{day})$, then recommend stopping

- If $<3$ preovulatory follicles, then discuss whether to continue or stop with patient

- If projected E2 day of hCG would be > 12,000 pM, then discuss coasting with doctor

- If abdominal discomfort, then doctor to discuss stopping with patient

\section{TRIGGER}

- Trigger ovulation with $250 \mu \mathrm{g}$ Ovidrel and stop Buserelin, when:

○ 2 follicles $>=20 \mathrm{~mm}$, or 3 follicles $>=18 \mathrm{~mm}, \mathrm{OR}$

○ Coasted and E2 falls to $<10,000 \mathrm{pM}$

- If need to adjust trigger day to avoid a weekend, trigger 1 day earlier rather than 1 day later. 
- Do not give hCG trigger if E2 > 20,000 pM

- If the E2 rise is significantly steeper than the standard line on the tracking sheet in the days leading to trigger, then a doctor must make the decision whether or not to trigger.

OPU (= luteal day 0$)$

- $36 \mathrm{~h}$ after hCG trigger

- Premedication of 2 Panadol tablets (500 mg each).

- Other medication requires doctor's prescription.

\section{EMBRYO REPLACEMENT}

- At least $45 \mathrm{~h}$ after insemination in IVF, $43 \mathrm{~h}$ after injection in ICSI insemination

- If E2 > 12,000 pM day of hCG trigger or >=20 eggs at OPU, then freeze all embryos

- If 15-20 eggs at OPU, then doctor's discretion whether to freeze all embryos

\section{LUTEAL SUPPORT AND MONITORING}

- Utrogestan intravaginal $2 \times 100 \mathrm{mg} 3 x$ / day from luteal day 1

- HCG test luteal day14

- If hCG >= $5 \mathrm{iu} / \mathrm{l}$ day 14 , then consider pregnant, and do E2, P4, hCG day 18

- HCG result day 18:

- If $\mathrm{P} 4>80 \mathrm{nM}$, reduce to $100 \mathrm{mg}$ Utrogestan $3 \mathrm{x} /$ day for 4 days, then stop.

- If P4 $<80 \mathrm{nM}$, stay on Utrogestan until scan with foetal heart at 10 weeks, then reduce to $100 \mathrm{mg}$ Utrogestan $3 x$ / day for 4 days then stop.

- Further monitoring at patient's request as early pregnancy monitoring service OR continue monitoring if any concern about possible ectopic pregnancy. 


\section{APPENDIX 3.1}

\section{Title ICSI Protocol}

Author Dr. Bert Stewart, Fertility Associates Ltd.

Purpose To describe the method used for ICSI

Scope Laboratory staff

Responsibility Laboratory Team Leader

Materials

Equilibrated G-IVF+ in labelled Nunc 4-well dishes in $\mathrm{CO}_{2}$ incubator.

Equilibrated G-IVF+ in labelled organ culture dishes in $\mathrm{CO}_{2}$ incubator

HYASE diluted to 30IU/ml with G-MOPS+

OVOIL

$10 \%$ polyvinylpyrrolidone solution (ICSI, Vitrolife)

$5 \mathrm{ml}$ pipette (Falcon 357543 )

Nunc 4-well dishes (44444).

Petri dishes (Falcon 353652).

Fire polished, washed, sterilized Pasteur pipette.

Long drawn Pasteur pipette.

Denudation pipette.

ICSI injection pipette

Oocyte holding pipette.

\section{Method}

\subsection{Day before Procedure}


Set up G-IVF+ OPU, rinse and culture dishes according to Laboratory Protocol, 5828.21

\section{Procedure}

\subsection{Timing:}

Optimal injection time is $38-40 \mathrm{~h}$ post hCG (2-4 hours post OPU).

On the Day of the procedure, dishes should be set up for denudation of oocytes and the ICSI procedure itself.

\subsection{Hyaluronidase dishes}

One Hyaluronidase dish should be made for each 'batch' of oocytes to be injected. (see Denudation and Injection of Oocytes below)

3.1 Nunc 4-well dishes (44444) are labelled with the patient's name.

3.2 Lines are drawn on the base of the dish connecting wells 1 to 2,2 to 4 and 4 to 3 .

3.3 Well 1 is highlighted by drawing a ring around it.

$3.40 .5 \mathrm{ml}$ of HYASE solution (2.9ml G-MOPS+ added to $0.1 \mathrm{ml}$ HYASE concentrate) is placed in well 1.

$3.50 .5 \mathrm{ml} \mathrm{G-MOPS}+$ is placed in each of the other 3 wells.

3.6 All wells are covered with $0.4 \mathrm{ml}$ OVOIL.

3.7 The dish (denudation) is equilibrated to $37^{\circ} \mathrm{C}$ in air.

\subsection{ICSI dishes}

1 or 2 injection dishes should be set up depending on the number of oocytes, see Denudation and Injection of Oocytes below.

4.1 Using a micro-pipettor place a 5 $\mu 1$ drop of PVP in the centre of the Petri dish (353652).

4.2 Arrange sufficient drops of G-MOPS+ $(5 \mu \mathrm{l})$ around the central drop.

4.3 Cover with $4.5 \mathrm{ml}$ oil. 
4.4 Immediately suck out G-MOPS+ drops from under the oil and replace with $10 \mu \mathrm{G}$ MOPS+.

4.5 Leave the central $5 \mu 1$ PVP drop. Equilibrate to $37^{\circ} \mathrm{C}$ in air.

4.6 Set up manipulators ready for injection.

\section{Denudation and Injection of Oocytes}

To minimize stress to oocytes through exposure to sub-optimal conditions, total time out of the incubator should not exceed 30 minutes. Only remove as many oocytes from the OPU dish as can be processed through denudation and injection in this time. Number will vary according to ease of denudation and quality/quantity of sperm available for injection.

\subsection{Denudation of Oocytes:}

Oocytes must first be stripped of their cumulus and coronal cells to assess nuclear maturation and aid visualisation of the injection procedure. This is achieved through a combination of exposure to hyaluronidase and mechanical disruption. Hyaluronidase is a toxic enzyme, so oocytes should be exposed for as short a time as is possible. No more than one minute is recommended.

5.1 FA: The OPU dish containing the patient's oocytes is removed from the incubator along with the denudation dish and placed on the heated stage of a dissecting microscope.

OFS: The OPU dish containing the patient's oocytes is removed from the incubator along with the denudation dish and placed in the IVF chamber with the $\mathrm{CO} 2$ turned off.

Using a fire polished Pasteur, transfer oocytes to well 1 of the denudation dish. The OPU dish is immediately replaced in the $\mathrm{CO}_{2}$ incubator unless another is available.

5.2 With the Pasteur, gently aspirate the oocytes for 5-10 seconds (the aim is to remove the bulk of the cumulus cells. Hyaluronidase will not disrupt the junctions that hold the coronal cells to the zona pellucida). Using a long drawn Pasteur or flexipet (OFS), remove the oocytes from the hyaluronidase and rinse in well 2. Using a new long drawn Pasteur or flexipet (OFS), move the oocytes to well 4 and gently rinse. 
5.3 Assist removal of remaining cumulus/coronal cells by gently aspirating with a Cook Flexipet/Swemed Denudation Pipette, and transfer denuded oocytes into final well of clean GMOPS+. When all oocytes are denuded, place in $1^{\text {st }}$ injection dish.

\subsection{Sperm Injection Procedure}

6.1 Take patient's prepared sperm sample from incubator, consciously identifying patient's name and checking against Semen Preparation Sheet. Place in rack in workstation. Transcribe script from tube onto IVF tracking sheet.

6.2 Using a micropipettor and new sterile tip, add $\sim 1 \mu 1$ of prepared sperm sample to the centre of the PVP drop. (For prepared samples with less than 500,000 motile sperm per ml, 1-5 $\mu 1$ of sample should be added to a peripheral G-MOPS+ droplet to allow 'swim-out').

6.3 Inject oocytes as per normal practice.

6.4 Remove patient's 'post-ICSI rinse/culture dish' from $\mathrm{CO}_{2}$ incubator. Gently rinse and move oocytes through each of first row of rinse drops and finally hold in groups (up to 4 per microdrop) in the mid row.

6.4 If there are more oocytes to inject, return rinse dish to $\mathrm{Minc} / \mathrm{CO}_{2}$ incubator.

6.6 Repeat above stages 5.0-6.5 with new denudation dish until all oocytes are denuded. 


\section{APPENDIX 3.2}

Title $\quad$ Fertilisation Check

Author Dr. Bert Stewart, Fertility Associates Ltd.

Purpose To describe the process of checking fertilisation

Scope Laboratory staff

Responsibility Laboratory Team Leader

\section{Fertilisation Check (2006)}

All oocytes (IVF and ICSI) should be checked for the presence of pronuclei between 14 and 18 hours post insemination; optimal is $16 \mathrm{hrs}$.

\section{Materials}

Equilibrated G1v5 culture microdrops in labelled Petri dishes (Falcon 353652) in $\mathrm{CO}_{2}$ incubator, (prepared day before fertilisation check).

Oocyte denuding pipette and holder.

Long drawn Pasteur pipette.

\section{Afternoon before fertilisation check}

1.1 Check patient tracking sheet for expected number of embryos. Label lid and base of Petri dish with patient's first name, surname and file number. Label lid with D1 G1v5.)

1.2 Depending on number of inseminated/injected oocytes, make up to three rows of three microdrops $(20 \mu 1)$. Cover with $7 \mathrm{ml}$ OVOIL. Remove all drops and replace with $40 \mu 1$ fresh G1v5. Place in $\mathrm{CO}_{2}$ incubator overnight to equilibrate.

\section{Morning of fertilisation check}

3.1 Identify patient's tracking sheet and fill in Date, Time and hours after OPU on IVF sheet 2. 
3.2 Identify patient's culture dish containing oocytes in the incubator.

3.2.1 FAA, FAH, FAW: Place dish on heated stage of stereo microscope.

3.2.2 OFS: Place dish in IVF workstation.

3.3 For IVF: strip off adhering cumulus cells by gently drawing oocyte in and out of denudation pipette.

3.4 For IVF and ICSI, record: number of pronuclei, number of polar bodies, any other significant findings, e.g. vacuoles, uneven sized or separated pronuclei.

3.5 After all oocytes have been checked, remove patient's 'Rinse/culture" dish from $\mathrm{CO}_{2}$ incubator. First, move all normal 2PN zygotes into the first G1v5 rinse drop. Using a new, rinsed pipette, rinse through all three drops and group evenly (up to four per group) in the mid row of microdrops. Abnormal 2PN zygotes (separated or very uneven sized PN, vacuoles or large polar bodies) should be discarded; however, if retained, they should be rinsed as above and grouped together in a third row microdrop.

3.6 Oocytes with OPN or 1PN should be retained and re-examined at 22h post-insemination.

3.7 Oocytes with $>2 \mathrm{PN}$ should be discarded.

3.8 Return dish containing viable embryos to $\mathrm{CO}_{2}$ incubator. Check patient's consent before discarding non-viable oocytes/embryos.

3.9 Complete tracking sheets. 


\section{APPENDIX 3.3}

\section{Title Embryo grading and selection}

Author Dr. Bert Stewart, Fertility Associates Ltd.

Purpose To describe the steps for grading and selecting embryos

Scope Laboratory staff

Responsibility Laboratory Team Leader

\section{Embryo Grading and Selection (2005.1)}

Selection of the 'best' embryos for fresh replacement in an IVF cycle can have a major influence on the chance of conception. Embryos are assessed according to the rate and symmetry of their cleavage and translucency of their cytoplasm degree of fragmentation. It is also worth noting unusual characteristics that may impact on developmental potential.

Materials:

Stereo Microscope with heated stage (FAA, FAH, FAW), or in IVF workstation (OFS) ICSI microscope.

Washed, sterilised, finely drawn Pasteur- pipettes.

Patient's tracking sheets.

\section{Method:}

\section{Day 0. The oocyte}

In practice, assessment of abnormal oocyte phenotypes is limited to ICSI cycles where the denuded oocyte can be examined on the ICSI microscope. Features associated with poor developmental potential (on-going pregnancy, NOT embryo morphology) are;

- Very large first polar bodies,

- Cytoplasmic vacuoles (large or many),

- Very large perivitelline space.

Presence of any of these features at injection should be noted. 


\section{Day 1 morning}

A degree of negative selection can be made at the pronucleate stage.

Features visible on the stereo microscope and are indicative of extremely low implantation potential are:

- Pronuclei of obviously uneven size (smallest <50\% diameter of largest),

- Pronuclei not in contact,

At fertilisation check, the above poor prognosis features should be recorded.

The remaining 'normal' zygotes should be quickly checked on the ICSI microscope for:

- Multiple cytoplasmic vacuoles,

- Supernumerary (micro) pro-nuclei.

Any zygotes displaying these features should also be regarded as poor prognosis.

>2PN zygotes: these should be discarded.

$1 \mathrm{PN}$ zygotes and 0PN oocytes should be re-checked at $22 \mathrm{~h}$ and at $25 \mathrm{~h}$ post insemination. $2 \mathrm{PN}$ must be seen together at some point for these to be regarded as normally fertilised. If not, they should be discarded.

\section{Day 1 (25 hours post insemination/injection)}

All cultured embryos should be assessed for early cleavage or nuclear envelope breakdown at 25 hours post insemination.

3.1 Remove dish containing zygotes from $\mathrm{CO}_{2}$ incubator and place on heated stage of stereo microscope.

3.2 Score each zygote according to developmental stage, and record any untoward observations (e.g. fragmentation*).

3.3 Complete appropriate section of IVF sheet 2 in patient's tracking sheets.

*Some aspects are undoubtedly grossly abnormal, e.g. 3 or more cells. Others depend on degree, e.g. 10\% fragmentation may not be detrimental while $>30 \%$ probably is. 


\section{Day 2 (44h post insemination)}

On the ICSI microscope, check embryos for presence of multinucleated blastomeres. Each blastomere should have one visible nucleus (or no visible nucleus). The presence of 2 or more nuclei within a blastomere is abnormal, and greatly decreases the implantation potential of that embryo. Affected embryos should not be replaced (unless only embryos available) and should not be frozen. 4-cell embryos should be tetrahedral in orientation.

\section{Day 3 (68h post insemination)}

\section{Progressive development:}

Embryo cell number must increase between day 2 and day 3.

On ICSI microscope count cells and note any untoward features such as multi-nucleation vacuoles or highly asymmetrical cleavage.

Early Cleavers: Choose from the early cleavage group when possible (2>1) as long as they are $1^{\text {st }}$ or $2^{\text {nd }}$ choice stage appropriate.

Morphology: After initial selections above, chose embryos with highest morphological grades 5.

\section{Embryo Replacement (Day 3):}

The 'best' embryos for replacement should have none of the poor prognosis features described above at any stage then be chosen after consultation the 'embryo development' matrix available in each lab. 


\section{APPENDIX 4}

\section{Tables for recording COC and oocyte morphological features}

Cumulus mass morphological features

\begin{tabular}{|l|l|l|l|l|l|l|l|l|l|l|l|l|l|l|l|}
\hline Cumulus \# & 1 & 2 & 3 & 4 & 5 & 6 & 7 & 8 & 9 & 10 & 11 & 12 & 13 & 14 & 15 \\
\hline 0. No abnormalities & & & & & & & & & & & & & & & \\
\hline 1. Small Cumulus (Ball) & & & & & & & & & & & & & & & \\
\hline 2. Dark Cumulus & & & & & & & & & & & & & & & \\
\hline 3. Cumulus with dark patches & & & & & & & & & & & & & & & \\
\hline
\end{tabular}

\section{Oocyte morphological features}

\begin{tabular}{|l|l|l|l|l|l|l|l|l|l|l|l|l|l|l|l|}
\hline Oocyte \# & 1 & 2 & 3 & 4 & 5 & 6 & 7 & 8 & 9 & 10 & 11 & 12 & 13 & 14 & 15 \\
\hline 0. MII (mature) & & & & & & & & & & & & & & & \\
\hline 1. MI (immature) & & & & & & & & & & & & & & & \\
\hline 2. GV (immature) & & & & & & & & & & & & & & & \\
\hline 3. Abnormal & & & & & & & & & & & & & & & \\
\hline
\end{tabular}




\section{APPENDIX 5}

Table 5C: Summary showing the Pearson correlation coefficients and significance levels ( $\mathrm{P}$ values) between biological and treatment variables in the 68 women recruited in the four study groups.

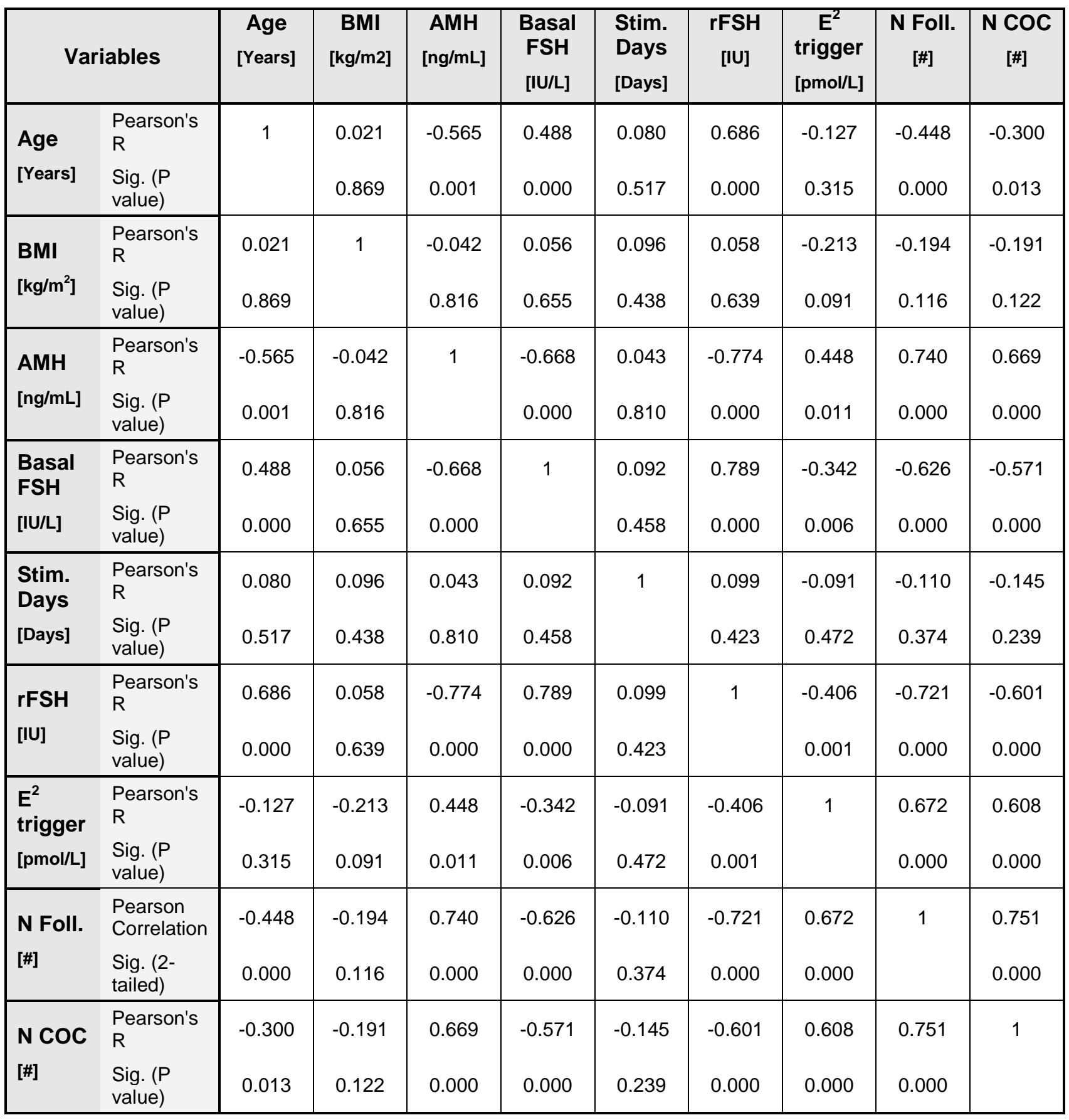




\section{APPENDIX 6}

A)

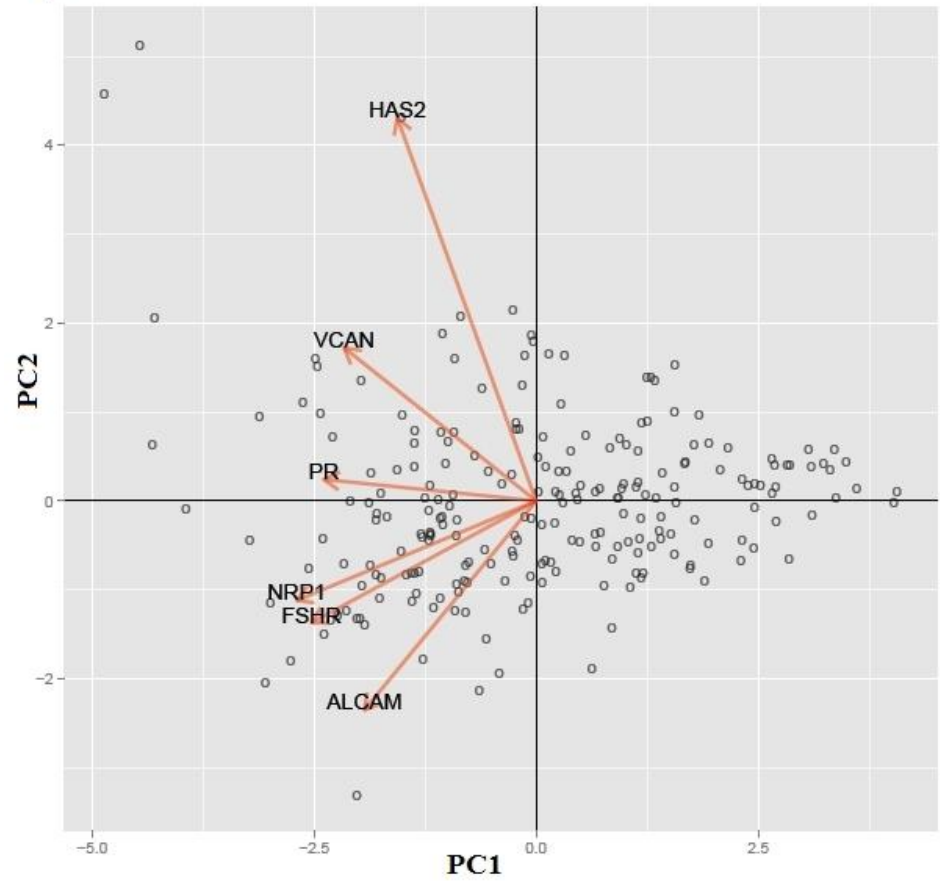

C)

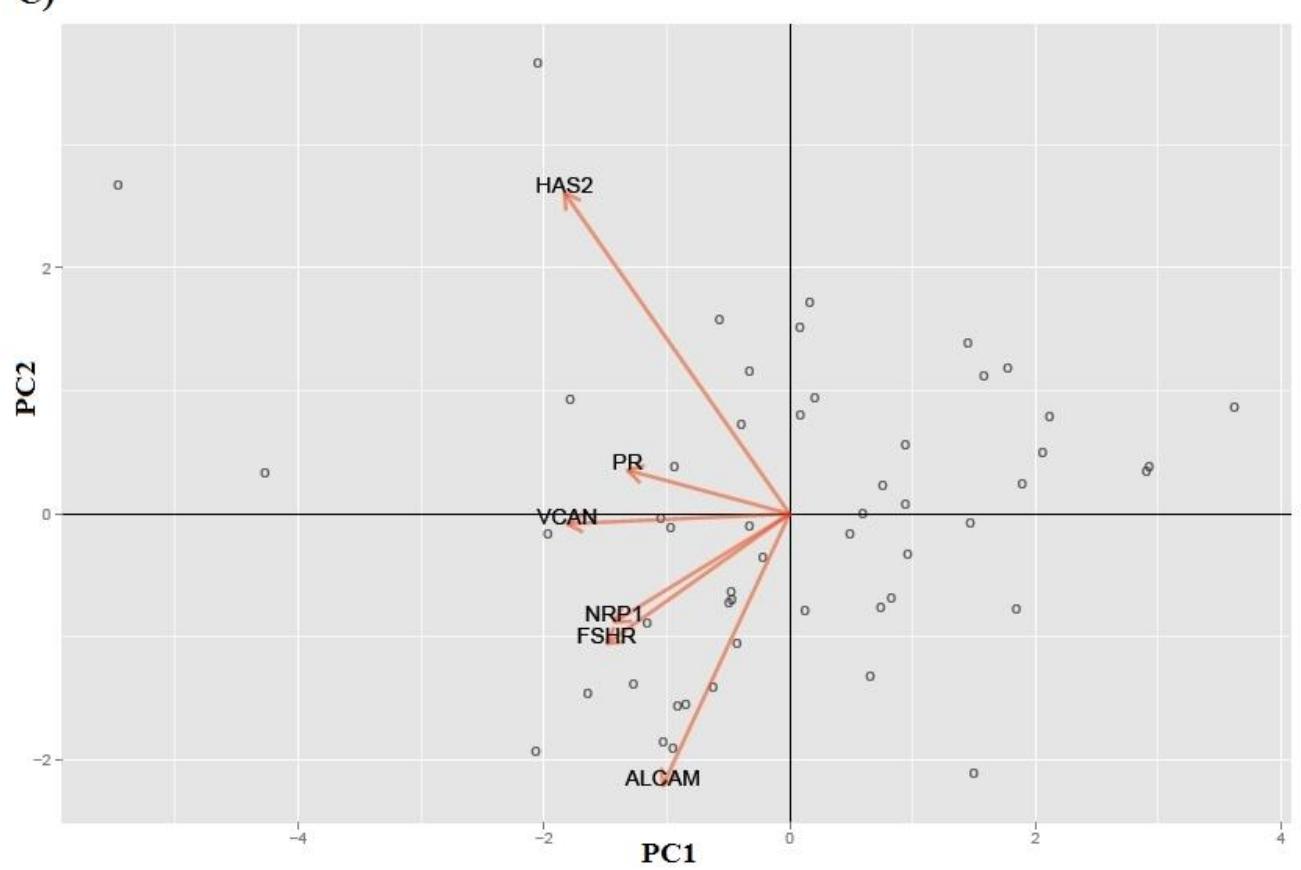

Figure 6I: Biplots of the six genes (HAS2, FSHR, ALCAM, VCAN, PR and NRP1) along the first and second principal component (PC1 and PC2). A) dots represent each $\mathrm{CC}$ mass $(\mathrm{N}=227)$ from Group 1 women. In this analysis PC1 accounted for 50.3\%, whilst PC2 accounted for $17.4 \%$ of 
the observed variance; B) dots represent each CC mass $(\mathrm{N}=175)$ associated with an oocyte with negative outcome (arrested embryo) from Group 1 women. In this analysis PC1 accounted for $53.2 \%$, and PC2 $15.4 \%$ of the observed variance; $\mathrm{C})$ dots represent each CC mass $(\mathrm{N}=52)$ associated with an oocyte that progressed to a good quality blastocyst from Group 1 women. In this analysis PC1 accounted for $42.5 \%$ and PC2 $22.1 \%$ of the observed variance. The closer the dots, the more similar the gene expression pattern is for individual $\mathrm{CC}$ masses according to the two principal components. The closer the arrows to each other, the more the gene expressions (normalized values) are correlated. 
Figure 7C (full data set)

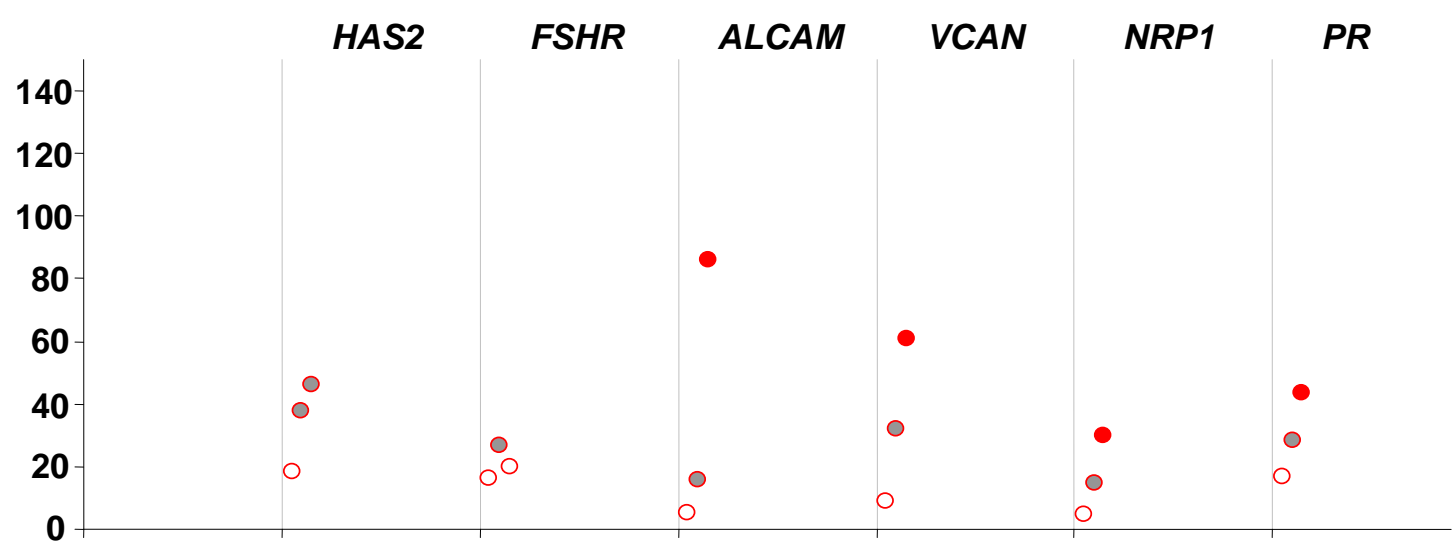

Patient \#1

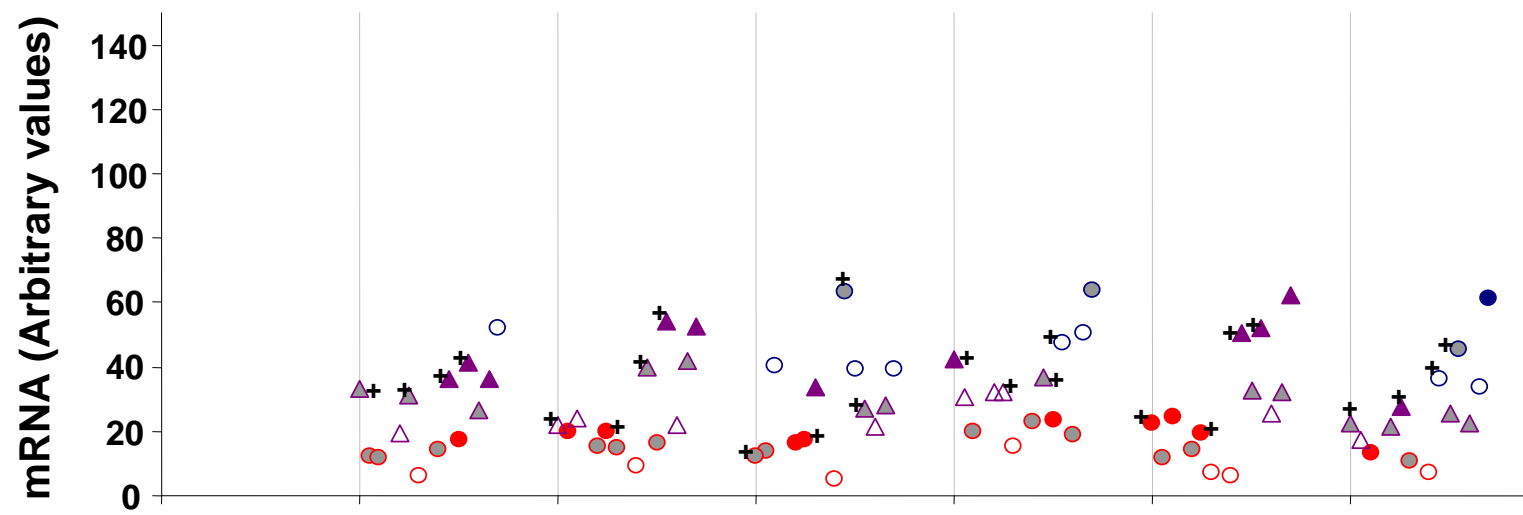

Patient \#2

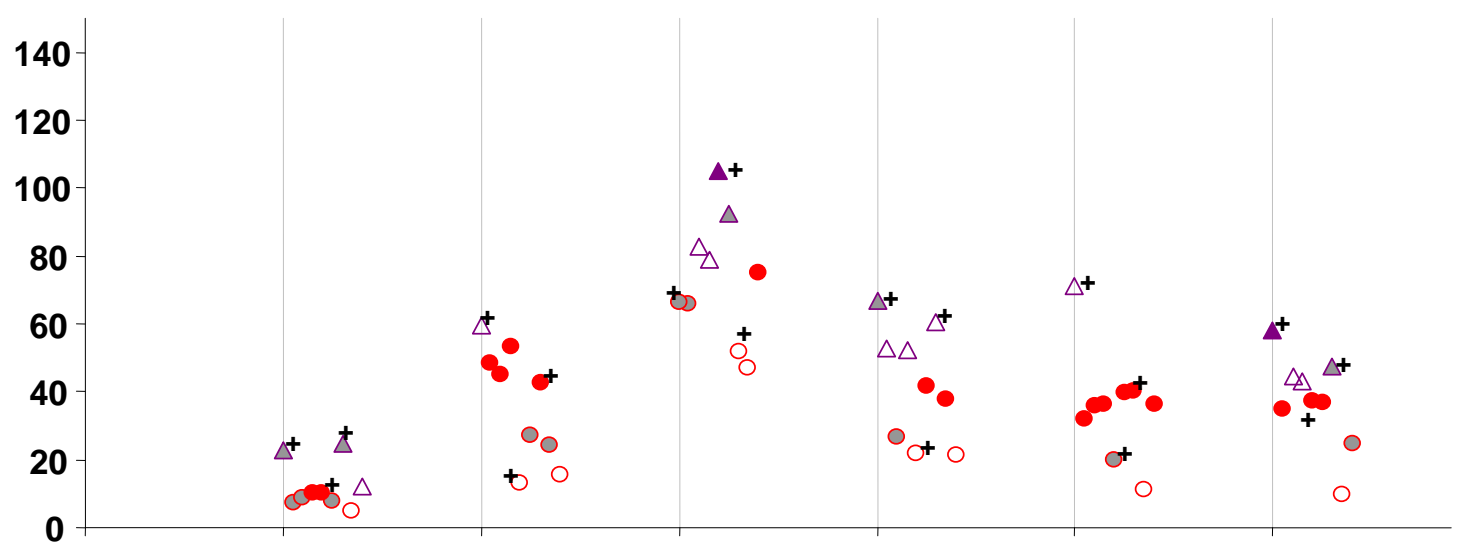

Patient \#3 


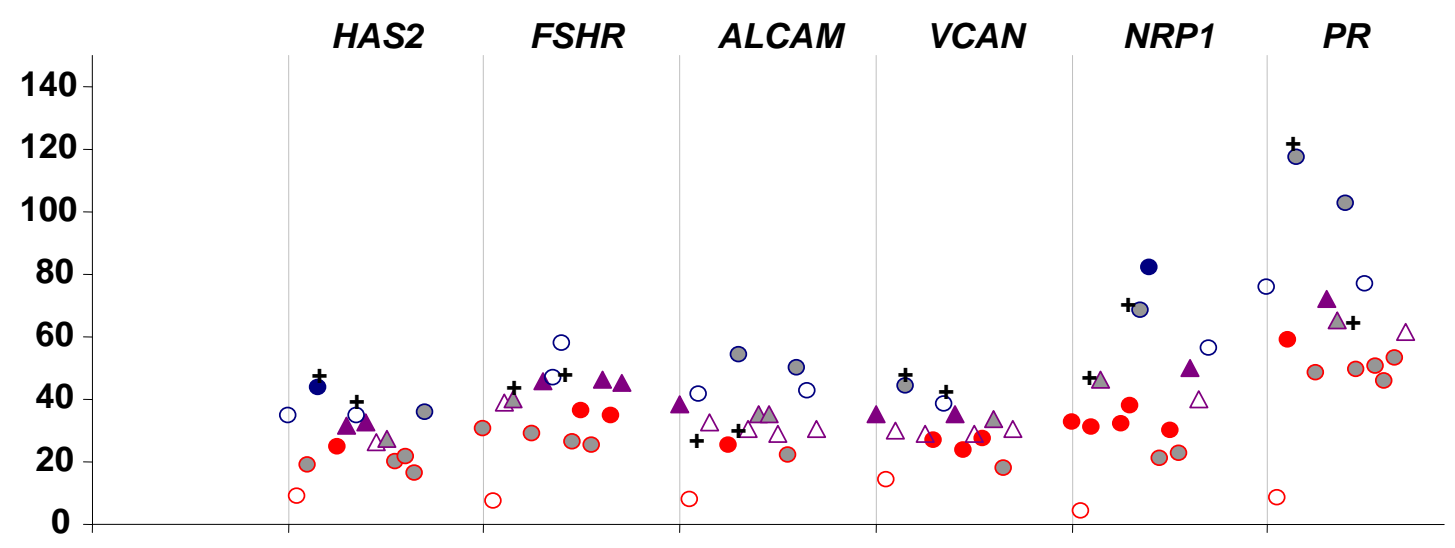

Patient \#4

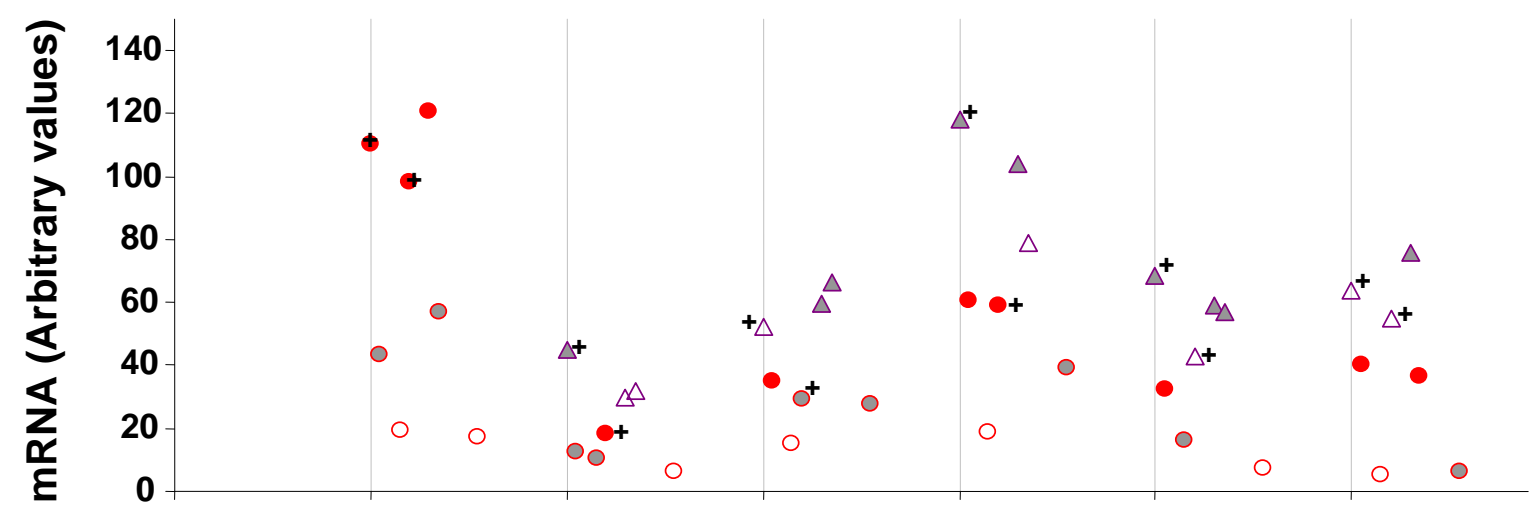

Patient \#5

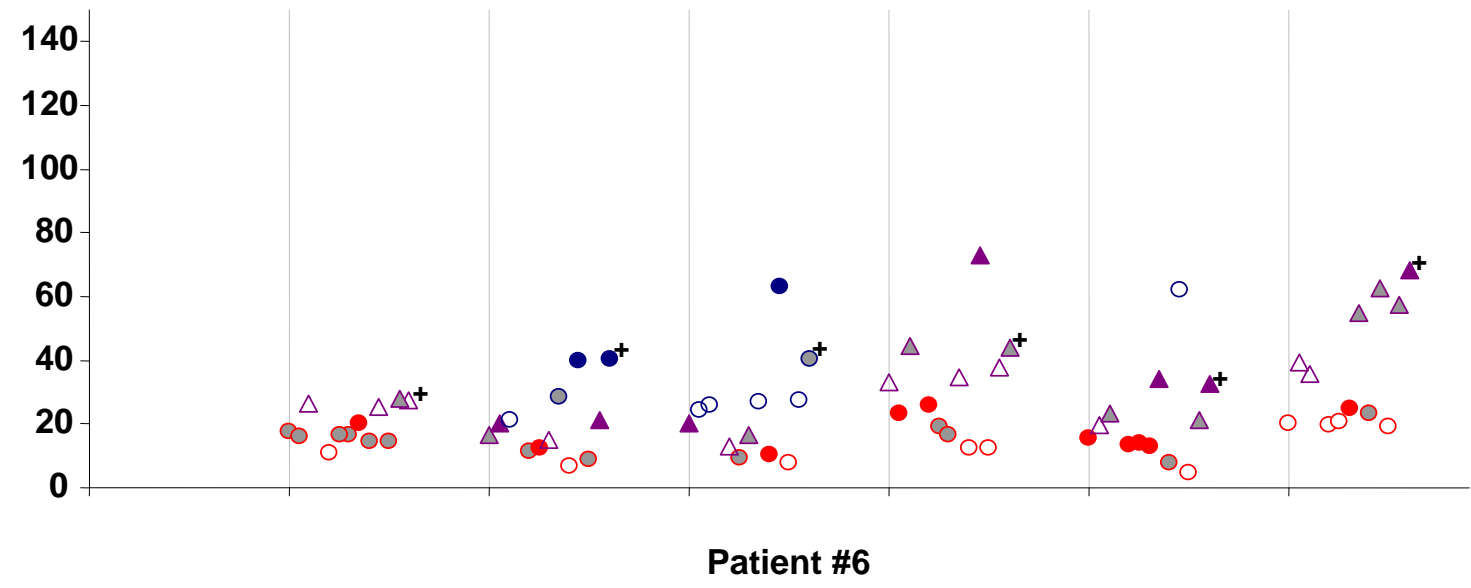




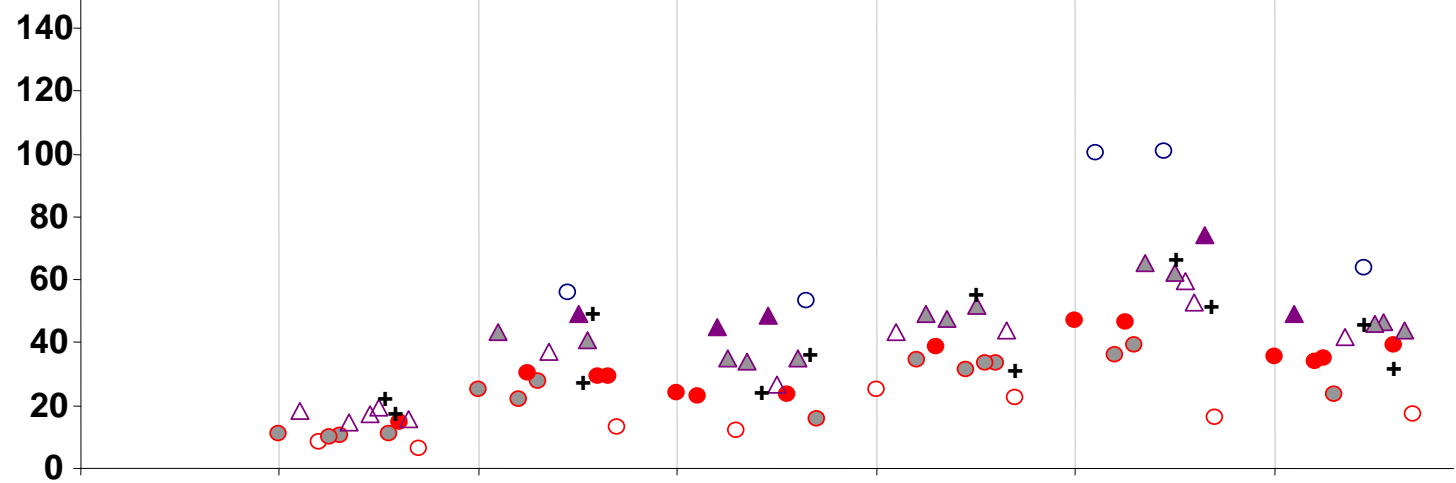

\section{Patient \#7}
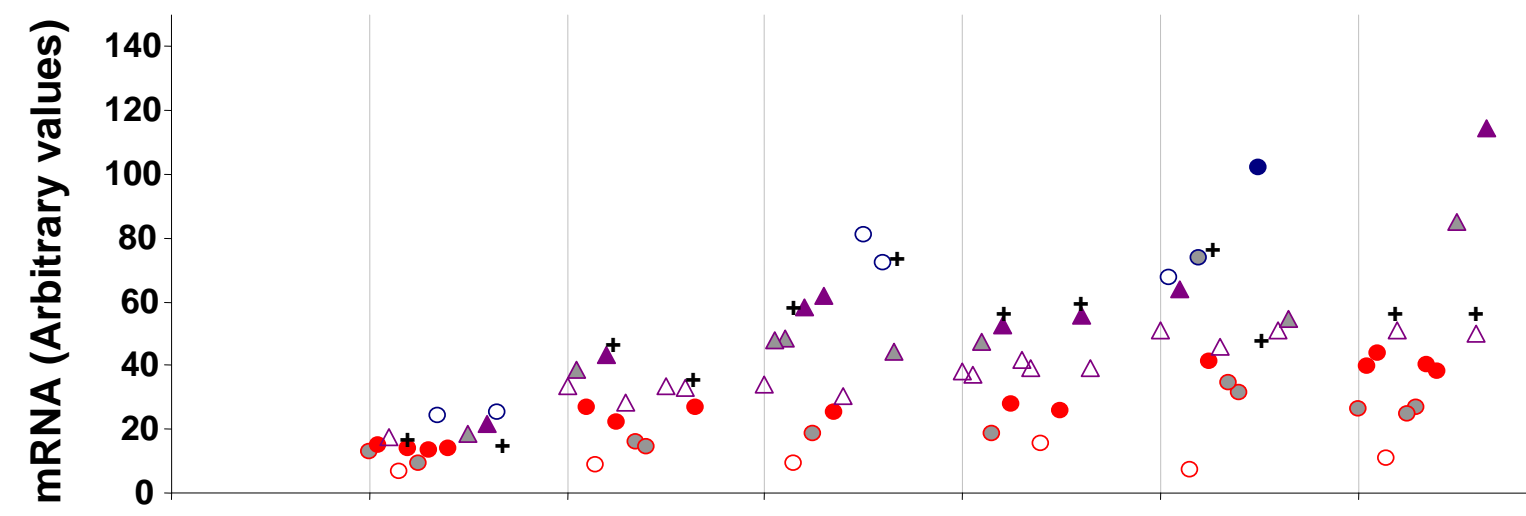

\section{Patient \#8}

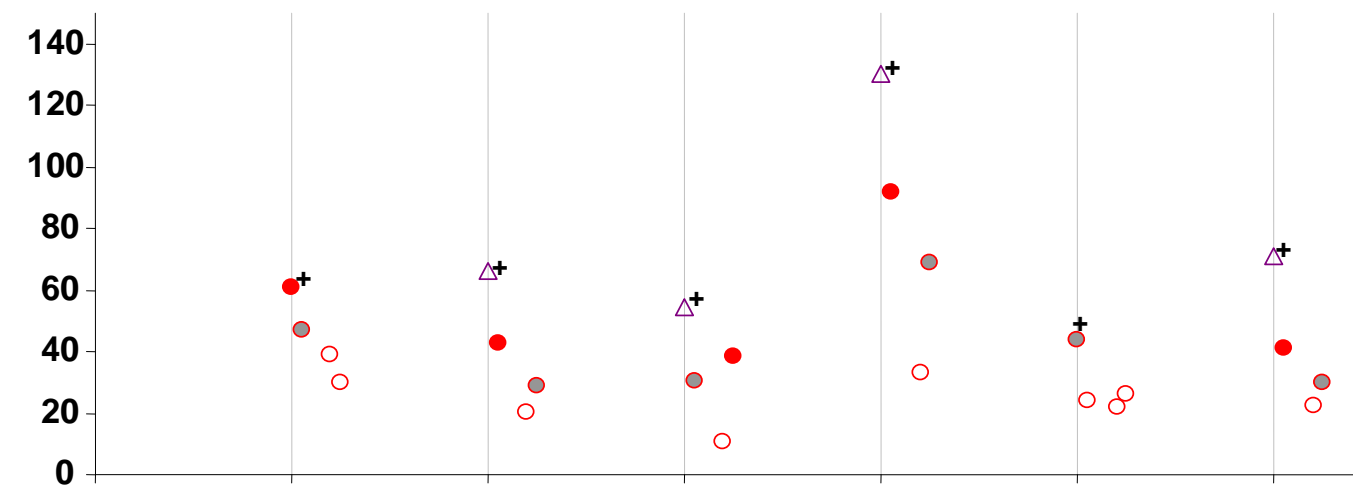

\section{Patient \#9}




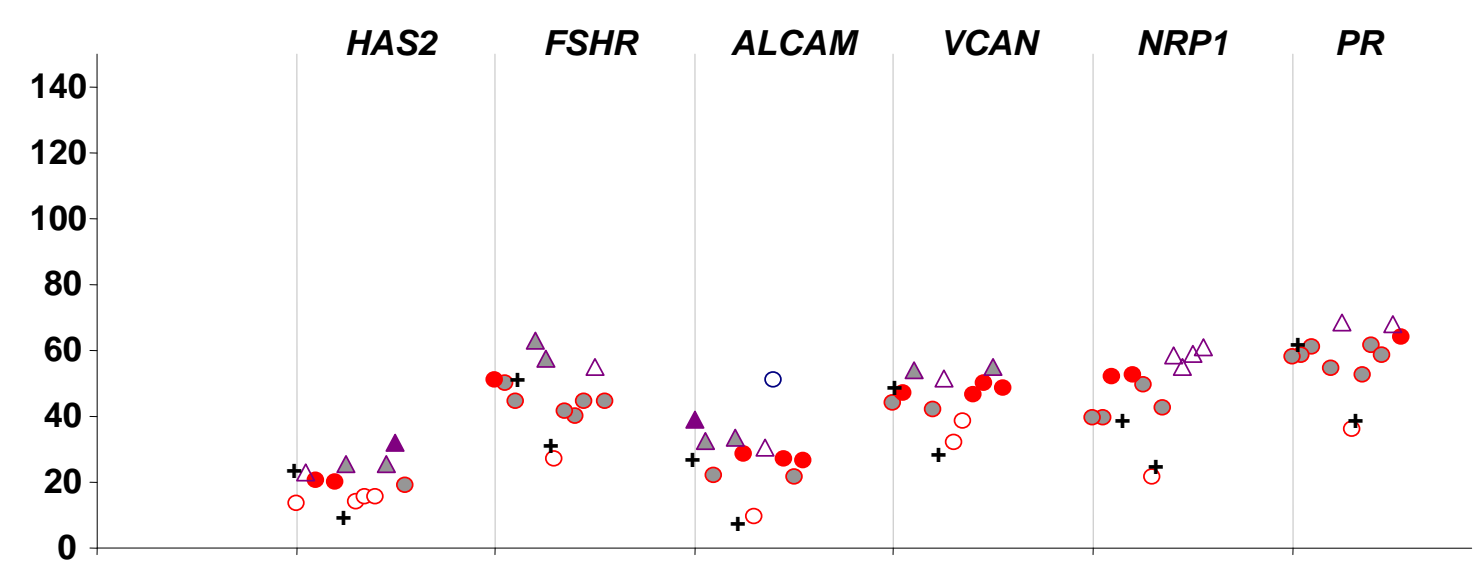

\section{Patient \#10}
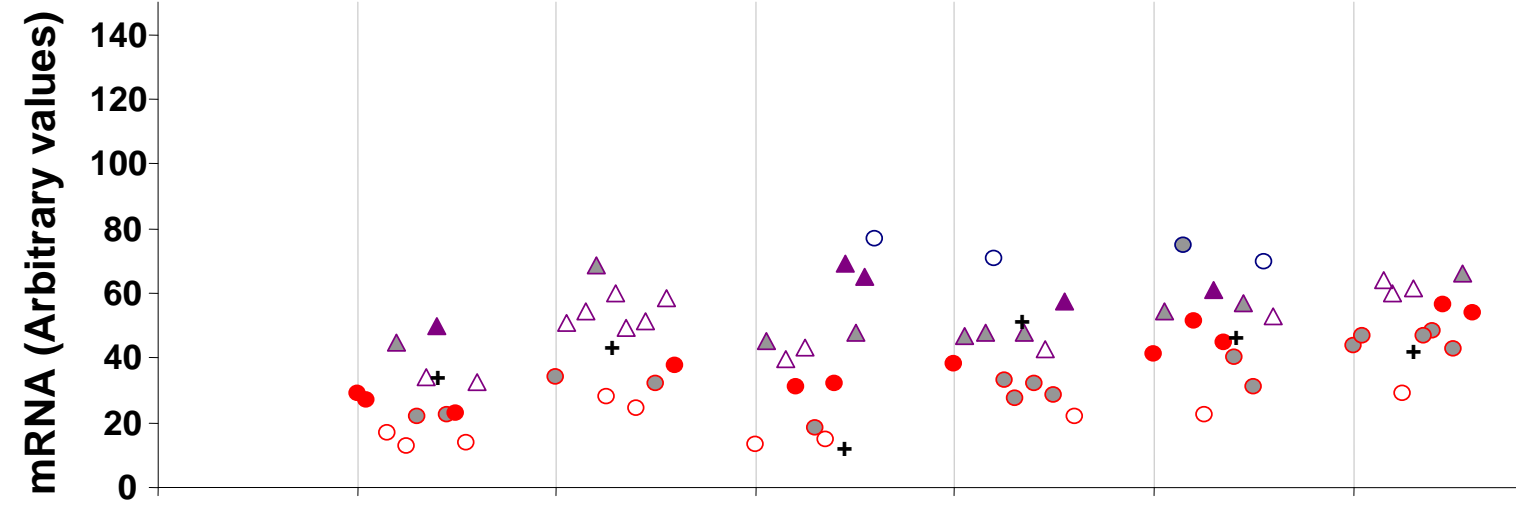

Patient \#11

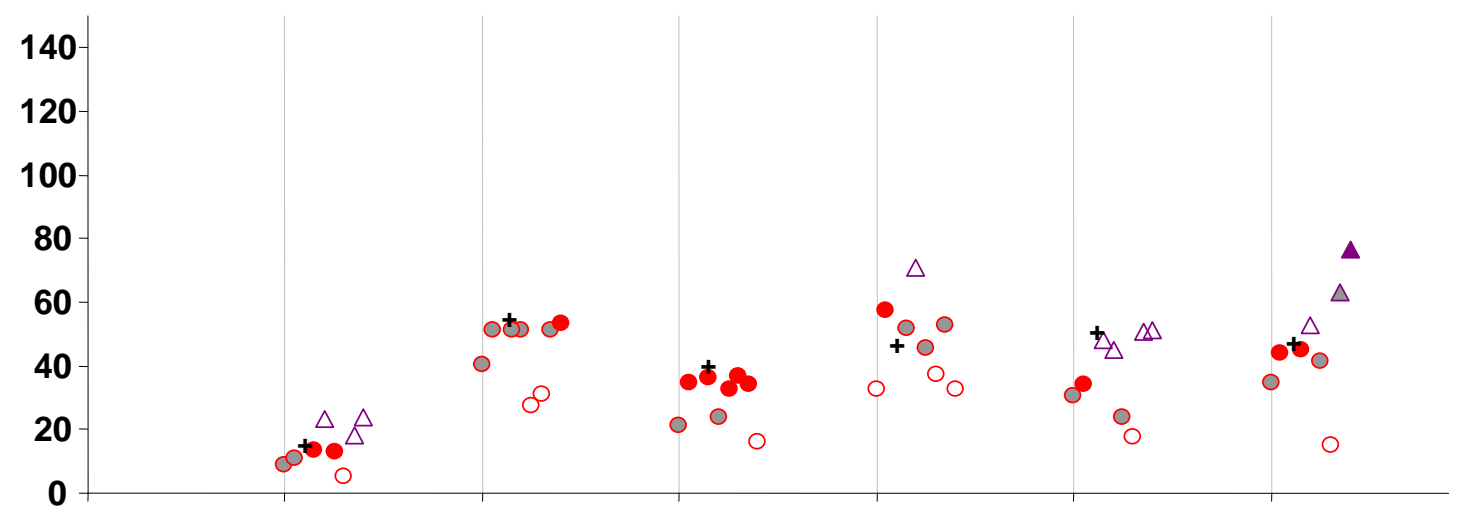

Patient \#12 


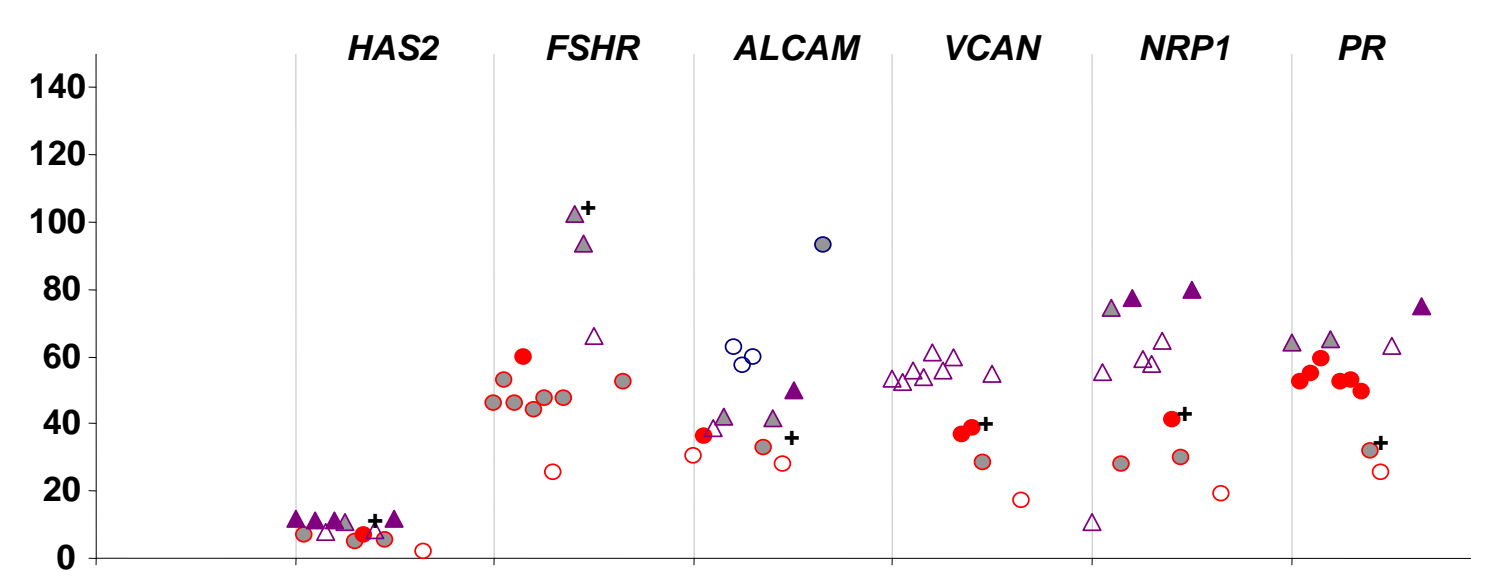

\section{Patient \#13}
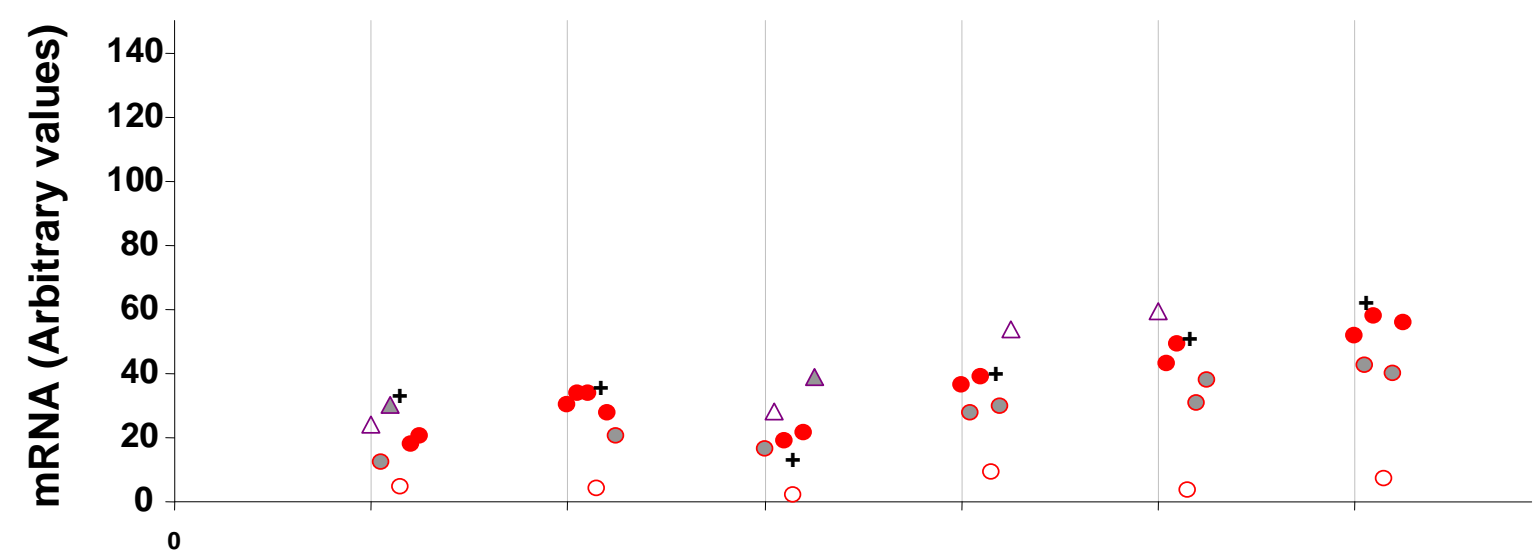

Patient \#14

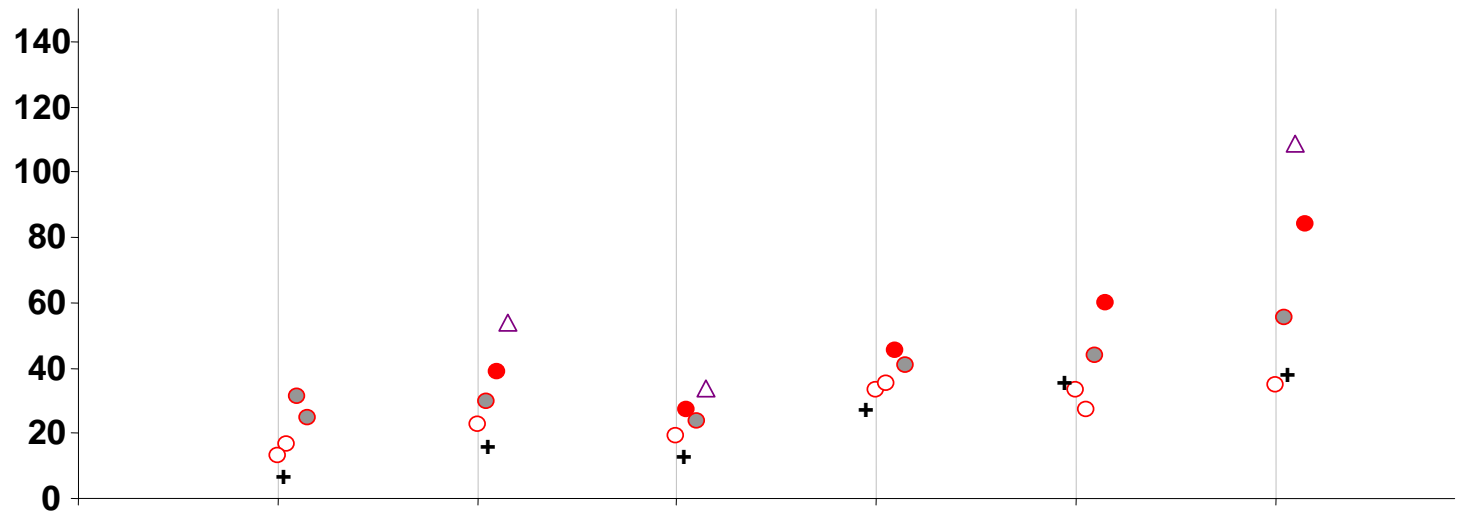

Patient \#15 


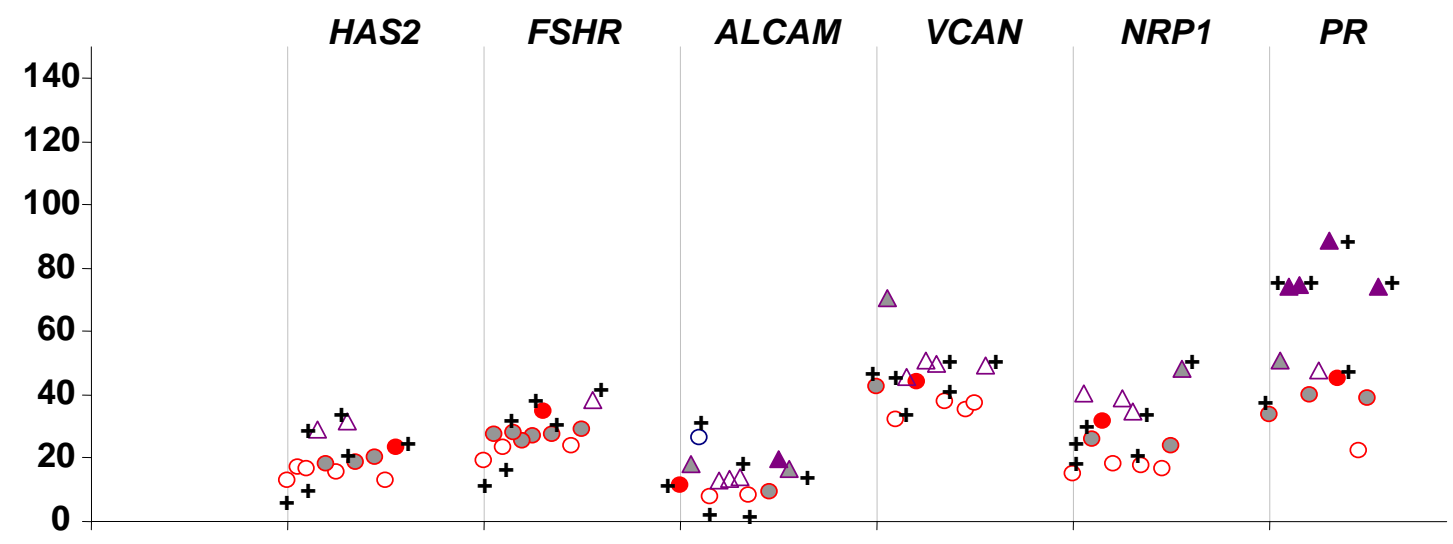

Patient \#16
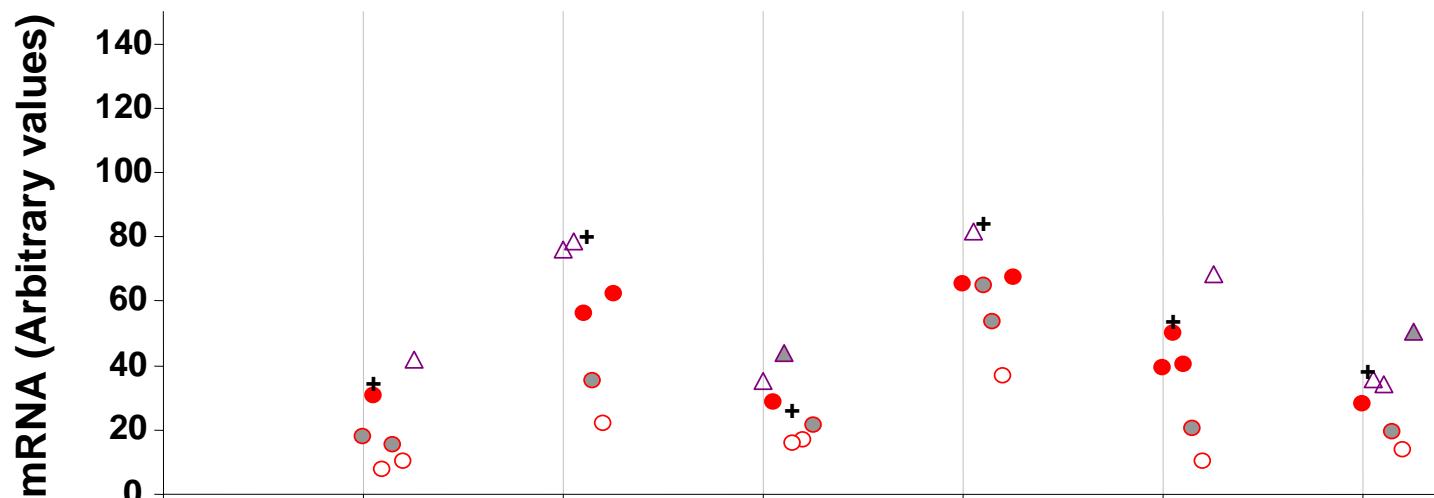

Patient \#17

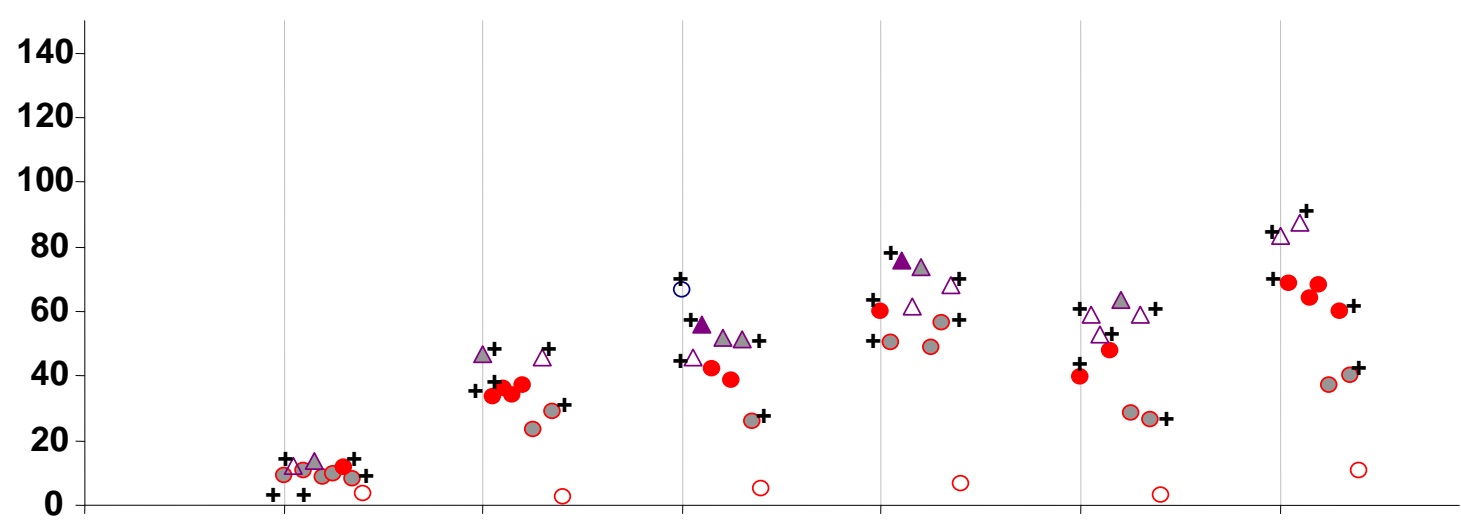

Patient \#18 


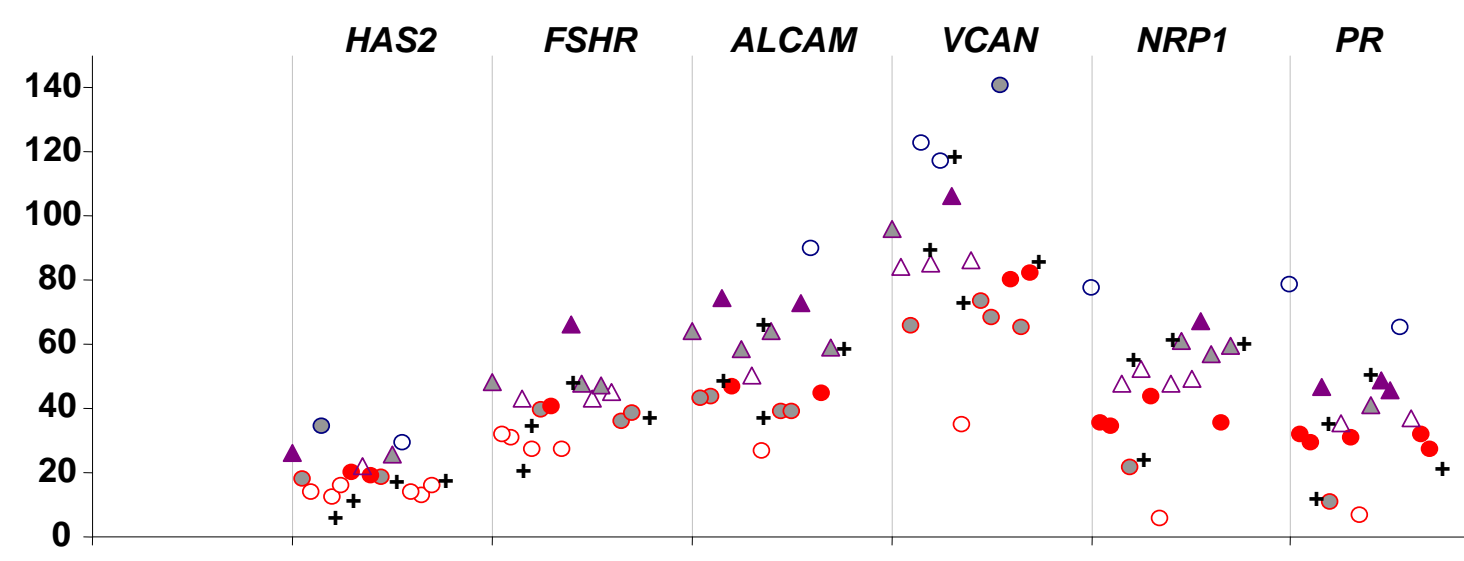

Patient \#19

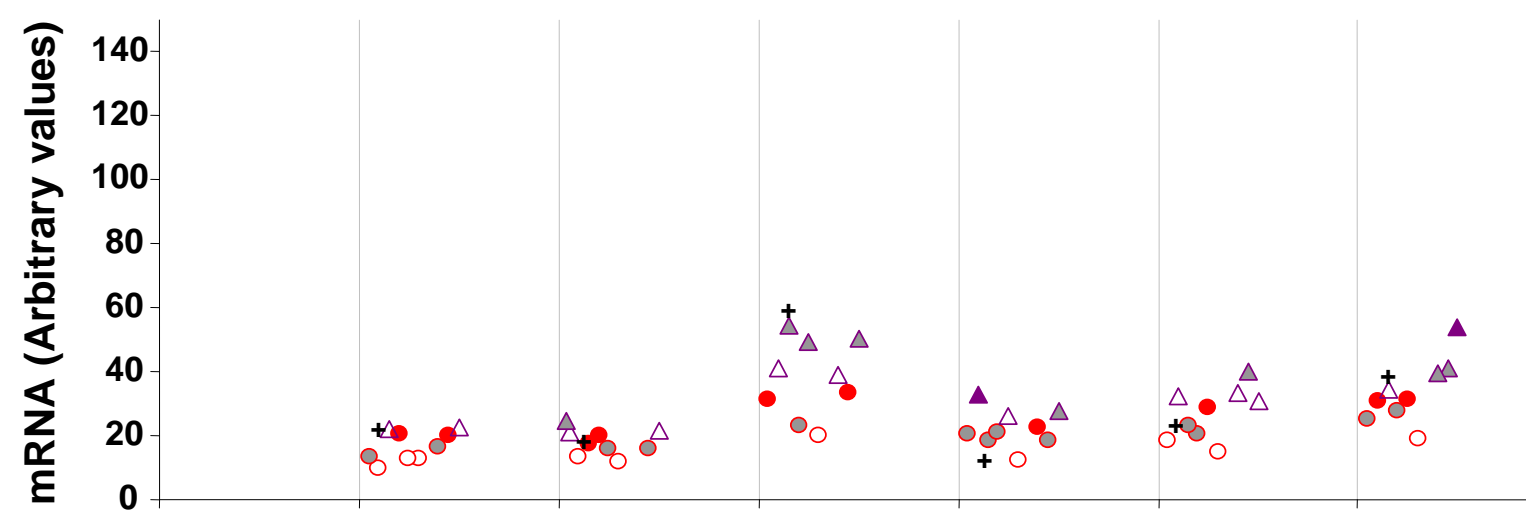

Patient \#20

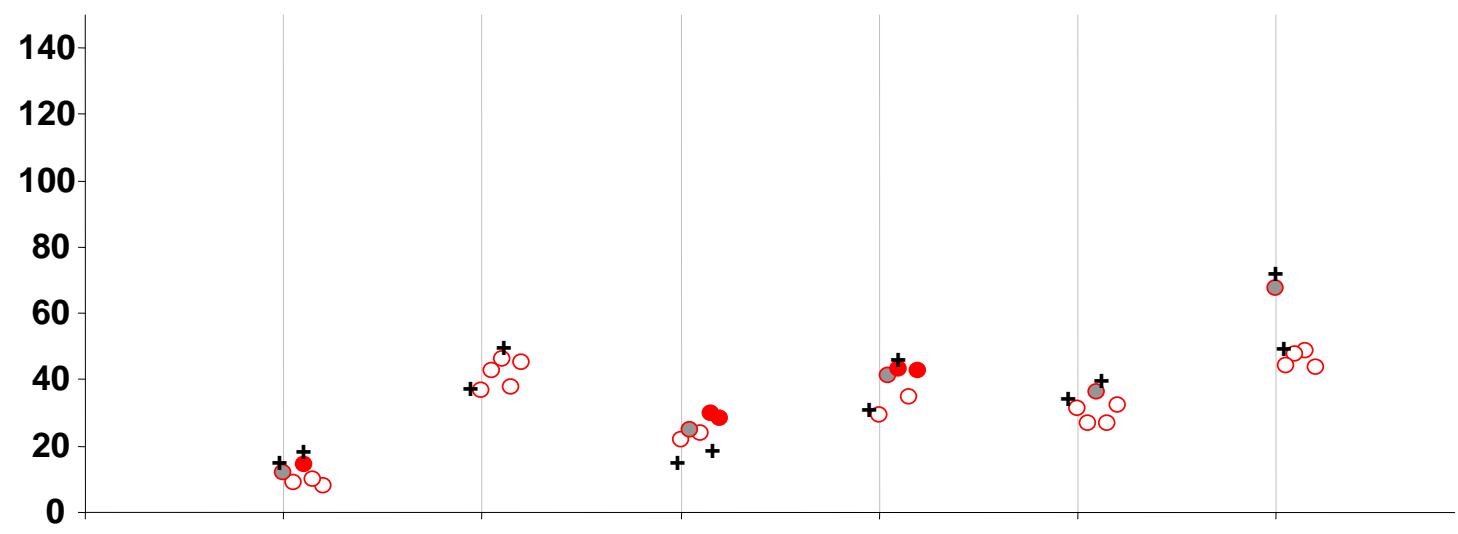

Patient \#21 


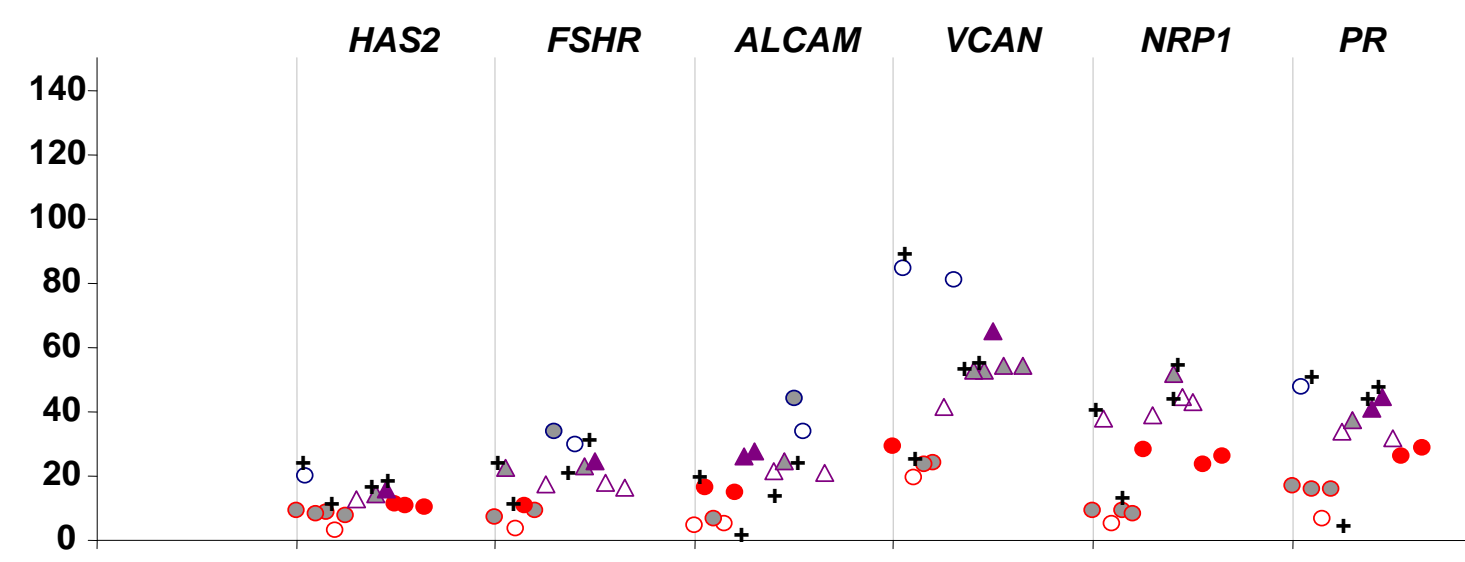

Patient \#22
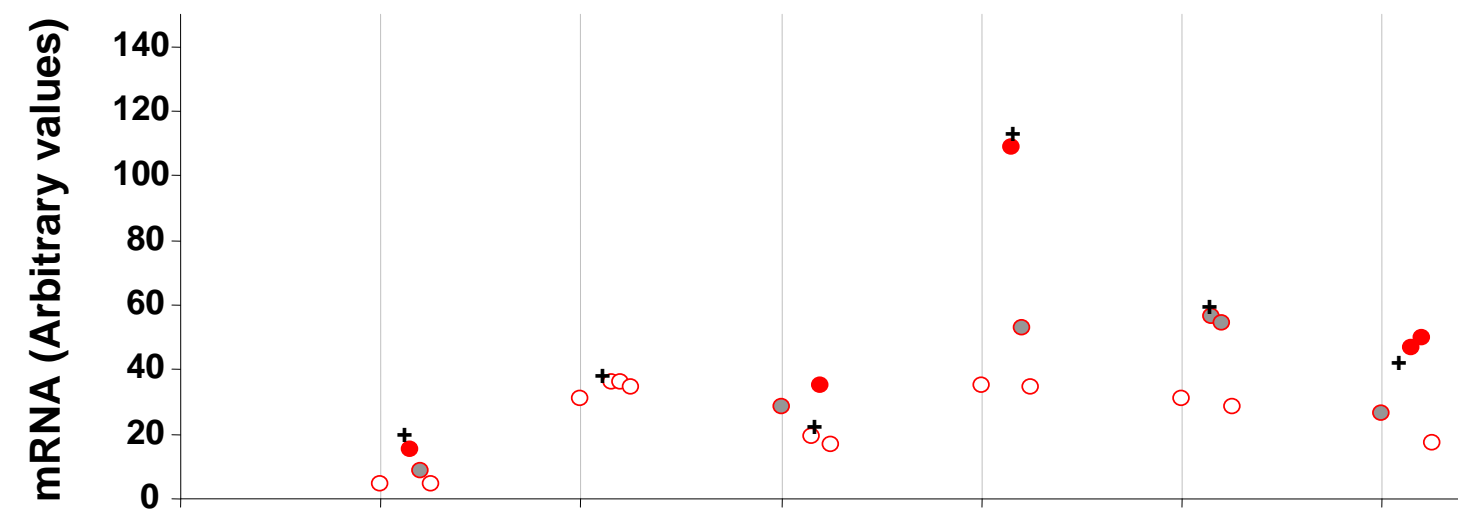

\section{Patient \#23}

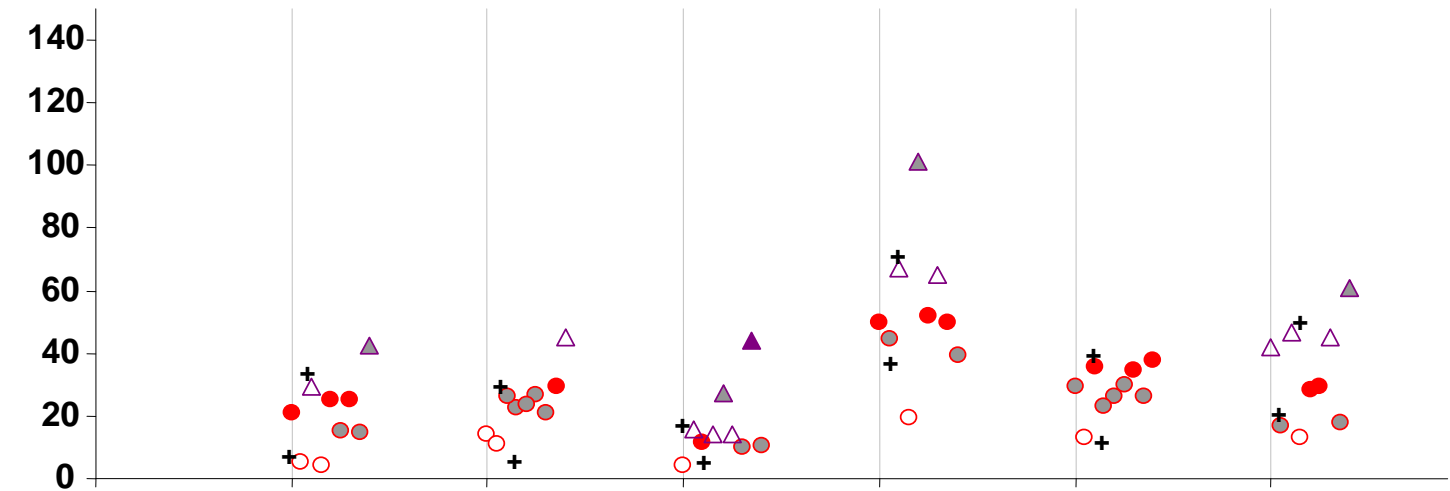

Patient \#24 


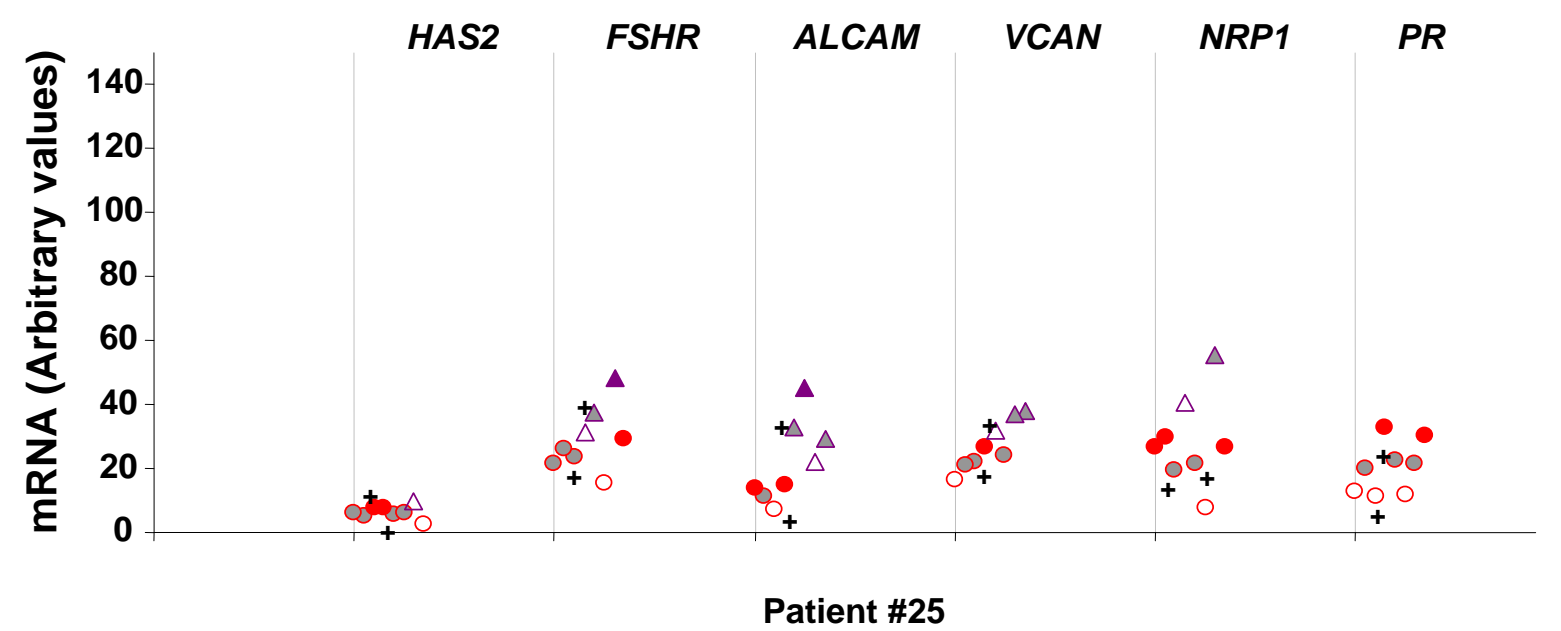

Figure 7C: Rankings of individual COC according to gene expression for patient \# 19. Individual $\mathrm{COC}$ are spread along the $\mathrm{X}$ axis for each candidate gene (HAS2, FSHR, ALCAM, VCAN, NRP1 and $P R$ ) from left to right according to their numerical order assigned at the time of egg collection. Expression results for each gene are separated by grey vertical lines. "+" marks are associated to dots (oocytes) with a positive outcome (blastocyst development and/or positive pregnancy). Different dots denote different ranks $(\circ=1, \diamond=2, \bullet=3, \Delta=4, \Delta=5, \Delta=6, \circ=7, \diamond=8$ and, $\bullet=9$ ). Gene expression is presented in $2^{-\Delta \mathrm{CT}}$ values (HAS2: 2000x $2^{-\Delta \mathrm{CT}}$, FSHR: 10000x $2^{-}$ $\triangle \mathrm{CT}$, ALCAM: 5000x $2^{-\triangle \mathrm{CT}}$, VCAN: 100x $2^{-\triangle \mathrm{CT}}$, NRP1: 500x $2^{-\triangle \mathrm{CT}}$ and, PR: 10000x $2^{-\Delta \mathrm{CT}}$ ) (data presented in this figure originates from figure 7B). 


\section{BIBLIOGRAPHY}

Abdalla, H.I. (2010) Are US results for assisted reproduction better than the rest? Is it a question of competence or policies? Reprod Biomed Online 21(5), 624-30

Aboulghar, M.A., Amin, Y.M., Al-Inany, H.G., Aboulghar, M.M., Mourad, L.M., Serour, G.I., and Mansour, R.T. (2008) Prospective randomized study comparing luteal phase support for ICSI patients up to the first ultrasound compared with an additional three weeks. Hum Reprod 23(4), 857-62

Adriaenssens, T., Segers, I., Wathlet, S., and Smitz, J. (2011) The cumulus cell gene expression profile of oocytes with different nuclear maturity and potential for blastocyst formation. $J$ Assist Reprod Genet 28(1), 31-40

Adriaenssens, T., Wathlet, S., Segers, I., Verheyen, G., De Vos, A., Van der Elst, J., Coucke, W., Devroey, P., and Smitz, J. (2010) Cumulus cell gene expression is associated with oocyte developmental quality and influenced by patient and treatment characteristics. Hum Reprod 25(5), 1259-70

Ahmed Ebbiary, N.A., Lenton, E.A., and Cooke, I.D. (1994) Hypothalamic-pituitary ageing: progressive increase in FSH and LH concentrations throughout the reproductive life in regularly menstruating women. Clin Endocrinol (Oxf) 41(2), 199-206

Al-Inany, H.G., Youssef, M.A., Aboulghar, M., Broekmans, F., Sterrenburg, M., Smit, J., and Abou-Setta, A.M. (2011) Gonadotrophin-releasing hormone antagonists for assisted reproductive technology. Cochrane Database Syst Rev(5), CD001750

Albertini, D.F., Combelles, C.M., Benecchi, E., and Carabatsos, M.J. (2001) Cellular basis for paracrine regulation of ovarian follicle development. Reproduction 121(5), 647-53

Almahbobi, G., Anderiesz, C., Hutchinson, P., McFarlane, J.R., Wood, C., and Trounson, A.O. (1996) Functional integrity of granulosa cells from polycystic ovaries. Clin Endocrinol (Oxf) 44(5), 571-80

ALPHA and ESHRE (2011) The Istanbul consensus workshop on embryo assessment: proceedings of an expert meeting. Hum Reprod 26(6), 1270-83

Anderson, R.A., Sciorio, R., Kinnell, H., Bayne, R.A., Thong, K.J., de Sousa, P.A., and Pickering, S. (2009) Cumulus gene expression as a predictor of human oocyte fertilisation, embryo development and competence to establish a pregnancy. Reproduction 138(4), 629-37 
Armstrong, D.T., and Dorrington, J.H. (1977) Estrogen biosynthesis in the ovaries and testes. Adv Sex Horm Res 3, 217-58

Assidi, M., Montag, M., Van der Ven, K., and Sirard, M.A. (2011) Biomarkers of human oocyte developmental competence expressed in cumulus cells before ICSI: a preliminary study. $J$ Assist Reprod Genet 28(2), 173-88

Assou, S., Haouzi, D., Mahmoud, K., Aouacheria, A., Guillemin, Y., Pantesco, V., Reme, T., Dechaud, H., De Vos, J., and Hamamah, S. (2008) A non-invasive test for assessing embryo potential by gene expression profiles of human cumulus cells: a proof of concept study. Mol Hum Reprod 14(12), 711-9

Asuncion, M., Calvo, R.M., San Millan, J.L., Sancho, J., Avila, S., and Escobar-Morreale, H.F. (2000) A prospective study of the prevalence of the polycystic ovary syndrome in unselected Caucasian women from Spain. J Clin Endocrinol Metab 85(7), 2434-8

Baker, T.G. (1963) A Quantitative and Cytological Study of Germ Cells in Human Ovaries. Proc R Soc Lond B Biol Sci 158, 417-33

Baker, T.G. (1972) Gametogenesis. Acta Endocrinol Suppl (Copenh) 166, 18-41

Balasch, J., Jove, I., Marquez, M., and Vanrell, J.A. (1991) Hormonal and histological evaluation of the luteal phase after combined GnRH-agonist/gonadotrophin treatment for superovulation and luteal phase support in in-vitro fertilization. Hum Reprod 6(7), 914-7

Bankowski, B.J., Lyerly, A.D., Faden, R.R., and Wallach, E.E. (2005) The social implications of embryo cryopreservation. Fertil Steril 84(4), 823-32

Barbieri, R.L., Smith, S., and Ryan, K.J. (1988) The role of hyperinsulinemia in the pathogenesis of ovarian hyperandrogenism. Fertil Steril 50(2), 197-212

Basille, C., Frydman, R., El Aly, A., Hesters, L., Fanchin, R., Tachdjian, G., Steffann, J., LeLorc'h, M., and Achour-Frydman, N. (2009) Preimplantation genetic diagnosis: state of the art. Eur J Obstet Gynecol Reprod Biol 145(1), 9-13

Biggers, J.D., Whittingham, D.G., and Donahue, R.P. (1967) The pattern of energy metabolism in the mouse oocyte and zygote. Proc Natl Acad Sci U S A 58(2), 560-7

Biljan, M.M., Mahutte, N.G., Dean, N., Hemmings, R., Bissonnette, F., and Tan, S.L. (1998) Pretreatment with an oral contraceptive is effective in reducing the incidence of functional ovarian cyst formation during pituitary suppression by gonadotropin-releasing hormone analogues. J Assist Reprod Genet 15(10), 599-604 
Bissonnette, F., Cohen, J., Collins, J., Cowan, L., Dale, S., Dill, S., Greene, C., Gysler, M., Hanck, B., Hughes, E., Leader, A., McDonald, S., Marrin, M., Martin, R., Min, J., Mortimer, D., Mortimer, S., Smith, J., Tsang, B., van Vugt, D., and Yuzpe, A. (2007) Incidence and complications of multiple gestation in Canada: proceedings of an expert meeting. Reprod Biomed Online 14(6), 773-90

Bouwmans, C.A., Lintsen, B.M., Eijkemans, M.J., Habbema, J.D., Braat, D.D., and Hakkaart, L. (2008) A detailed cost analysis of in vitro fertilization and intracytoplasmic sperm injection treatment. Fertil Steril 89(2), 331-41

Bowen, M.A., and Aruffo, A. (1999) Adhesion molecules, their receptors, and their regulation: analysis of CD6-activated leukocyte cell adhesion molecule (ALCAM/CD166) interactions. Transplant Proc 31(1-2), 795-6

Braw-Tal, R. (2002) The initiation of follicle growth: the oocyte or the somatic cells? Mol Cell Endocrinol 187(1-2), 11-8

Bromer, J.G., Sakkas, D., Siano, L.J., Benadiva, C.A., and Patrizio, P. (2009) Reproductive efficiency of women over the age of 40 and the low risk of multiple pregnancies. Reprod Biomed Online 19 Suppl 4, 4316

Brown, J.B. (1978) Pituitary control of ovarian function--concepts derived from gonadotrophin therapy. Aust N Z J Obstet Gynaecol 18(1), 46-54

Buccione, R., Schroeder, A.C., and Eppig, J.J. (1990a) Interactions between somatic cells and germ cells throughout mammalian oogenesis. Biol Reprod 43(4), 543-7

Buccione, R., Vanderhyden, B.C., Caron, P.J., and Eppig, J.J. (1990b) FSH-induced expansion of the mouse cumulus oophorus in vitro is dependent upon a specific factor(s) secreted by the oocyte. Dev Biol 138(1), 16-25

Byskov, A.G., Faddy, M.J., Lemmen, J.G., and Andersen, C.Y. (2005) Eggs forever? Differentiation 73(9-10), 438-46

Cahill, D.J., Prosser, C.J., Wardle, P.G., Ford, W.C., and Hull, M.G. (1994) Relative influence of serum follicle stimulating hormone, age and other factors on ovarian response to gonadotrophin stimulation. Br J Obstet Gynaecol 101(11), 999-1002

Cai, J., Lou, H.Y., Dong, M.Y., Lu, X.E., Zhu, Y.M., Gao, H.J., and Huang, H.F. (2007) Poor ovarian response to gonadotropin stimulation is associated with low expression of folliclestimulating hormone receptor in granulosa cells. Fertil Steril 87(6), 1350-6 
Caixeta, E.S., Ripamonte, P., Franco, M.M., Junior, J.B., and Dode, M.A. (2009) Effect of follicle size on mRNA expression in cumulus cells and oocytes of Bos indicus: an approach to identify marker genes for developmental competence. Reprod Fertil Dev 21(5), 655-64

Calle, A., Fernandez-Gonzalez, R., Ramos-Ibeas, P., Laguna-Barraza, R., Perez-Cerezales, S., Bermejo-Alvarez, P., Ramirez, M.A., and Gutierrez-Adan, A. (2012) Long-term and transgenerational effects of in vitro culture on mouse embryos. Theriogenology 77(4), 785-93

Carmina, E., and Lobo, R.A. (1999) Polycystic ovary syndrome (PCOS): arguably the most common endocrinopathy is associated with significant morbidity in women. $J$ Clin Endocrinol Metab 84(6), 1897-9

Catteau-Jonard, S., Jamin, S.P., Leclerc, A., Gonzales, J., Dewailly, D., and di Clemente, N. (2008) Anti-Mullerian hormone, its receptor, FSH receptor, and androgen receptor genes are overexpressed by granulosa cells from stimulated follicles in women with polycystic ovary syndrome. J Clin Endocrinol Metab 93(11), 4456-61

Chabbert Buffet, N., Djakoure, C., Maitre, S.C., and Bouchard, P. (1998) Regulation of the human menstrual cycle. Front Neuroendocrinol 19(3), 151-86

Chambers, G.M., Sullivan, E.A., Ishihara, O., Chapman, M.G., and Adamson, G.D. (2009) The economic impact of assisted reproductive technology: a review of selected developed countries. Fertil Steril 91(6), 2281-94

Chang, M.Y., Chiang, C.H., Hsieh, T.T., Soong, Y.K., and Hsu, K.H. (1998) Use of the antral follicle count to predict the outcome of assisted reproductive technologies. Fertil Steril 69(3), $505-10$

Chari, R., Lonergan, K.M., Pikor, L.A., Coe, B.P., Zhu, C.Q., Chan, T.H., MacAulay, C.E., Tsao, M.S., Lam, S., Ng, R.T., and Lam, W.L. (2010) A sequence-based approach to identify reference genes for gene expression analysis. BMC Med Genomics 3, 32

Chian, R.C., Huang, J.Y., Tan, S.L., Lucena, E., Saa, A., Rojas, A., Ruvalcaba Castellon, L.A., Garcia Amador, M.I., and Montoya Sarmiento, J.E. (2008) Obstetric and perinatal outcome in 200 infants conceived from vitrified oocytes. Reprod Biomed Online 16(5), 608-10

Cillo, F., Brevini, T.A., Antonini, S., Paffoni, A., Ragni, G., and Gandolfi, F. (2007) Association between human oocyte developmental competence and expression levels of some cumulus genes. Reproduction 134(5), 645-50

Clarke, G.N. (2006) A.R.T. and history, 1678-1978. Hum Reprod 21(7), 1645-50 
Collins, J. (2002) An international survey of the health economics of IVF and ICSI. Hum Reprod Update 8(3), 265-77

Corn, C.M., Hauser-Kronberger, C., Moser, M., Tews, G., and Ebner, T. (2005) Predictive value of cumulus cell apoptosis with regard to blastocyst development of corresponding gametes. Fertil Steril 84(3), 627-33

Coticchio, G., Sereni, E., Serrao, L., Mazzone, S., Iadarola, I., and Borini, A. (2004) What criteria for the definition of oocyte quality? Ann N Y Acad Sci 1034, 132-44

Crawford, J.L., and McNatty, K.P. (2012) The ratio of growth differentiation factor 9: bone morphogenetic protein 15 mRNA expression is tightly co-regulated and differs between species over a wide range of ovulation rates. Mol Cell Endocrinol 348(1), 339-43

Croxatto, H.B. (2002) Physiology of gamete and embryo transport through the fallopian tube. Reprod Biomed Online 4(2), 160-9

Cummins, J.M., Breen, T.M., Harrison, K.L., Shaw, J.M., Wilson, L.M., and Hennessey, J.F. (1986) A formula for scoring human embryo growth rates in in vitro fertilization: its value in predicting pregnancy and in comparison with visual estimates of embryo quality. $J$ In Vitro Fert Embryo Transf 3(5), 284-95

Damario, M.A., Barmat, L., Liu, H.C., Davis, O.K., and Rosenwaks, Z. (1997) Dual suppression with oral contraceptives and gonadotrophin releasing-hormone agonists improves in-vitro fertilization outcome in high responder patients. Hum Reprod 12(11), 2359-65

Daya, S. (2000) Gonadotropin releasing hormone agonist protocols for pituitary desensitization in in vitro fertilization and gamete intrafallopian transfer cycles. Cochrane Database Syst $\operatorname{Rev}(2), \mathrm{CD} 001299$

De Kretzer, D., Dennis, P., Hudson, B., Leeton, J., Lopata, A., Outch, K., Talbot, J., and Wood, C. (1973) Transfer of a human zygote. Lancet 2(7831), 728-9

De Sutter, P. (2006) Rational diagnosis and treatment in infertility. Best Pract Res Clin Obstet Gynaecol 20(5), 647-64

De Sutter, P., and Dhont, M. (2003) Poor response after hormonal stimulation for in vitro fertilization is not related to ovarian aging. Fertil Steril 79(6), 1294-8

Defronzo, R.A. (1979) Glucose intolerance and aging: evidence for tissue insensitivity to insulin. Diabetes 28(12), 1095-101 
Dekel, N., and Kraicer, P.F. (1978) Induction in vitro of mucification of rat cumulus oophorus by gonadotrophins and adenosine 3',5'-monophosphate. Endocrinology 102(6), 1797-802

Devoto, L., Fuentes, A., Kohen, P., Cespedes, P., Palomino, A., Pommer, R., Munoz, A., and Strauss, J.F., 3rd (2009) The human corpus luteum: life cycle and function in natural cycles. Fertil Steril 92(3), 1067-79

Devroey, P., Bourgain, C., Macklon, N.S., and Fauser, B.C. (2004) Reproductive biology and IVF: ovarian stimulation and endometrial receptivity. Trends Endocrinol Metab 15(2), 84-90

Dheda, K., Huggett, J.F., Bustin, S.A., Johnson, M.A., Rook, G., and Zumla, A. (2004) Validation of housekeeping genes for normalizing RNA expression in real-time PCR. Biotechniques 37(1), 112-4, 116, 118-9

Donahue, R.P., and Stern, S. (1968) Follicular cell support of oocyte maturation: production of pyruvate in vitro. J Reprod Fertil 17(2), 395-8

Durlinger, A.L., Visser, J.A., and Themmen, A.P. (2002) Regulation of ovarian function: the role of anti-Mullerian hormone. Reproduction 124(5), 601-9

Ebrahim, A., Rienhardt, G., Morris, S., Kruger, T.F., Lombard, C.J., and Van der Merwe, J.P. (1993) Follicle stimulating hormone levels on cycle day 3 predict ovulation stimulation response. J Assist Reprod Genet 10(2), 130-6

Edwards, R.G., Bavister, B.D., and Steptoe, P.C. (1969) Early stages of fertilization in vitro of human oocytes matured in vitro. Nature 221(5181), 632-5

Edwards, R.G., Lobo, R., and Bouchard, P. (1996) Time to revolutionize ovarian stimulation. Hum Reprod 11(5), 917-9

Edwards, R.G., Steptoe, P.C., and Purdy, J.M. (1970) Fertilization and cleavage in vitro of preovulator human oocytes. Nature 227(5265), 1307-9

Elective Services. The New Zealand Ministry of Health. (2011) www.electiveservices.govt.nz/pdfs/gynaecology-infertility.pdf (2005, date last accessed) el-Fouly, M.A., Cook, B., Nekola, M., and Nalbandov, A.V. (1970) Role of the ovum in follicular luteinization. Endocrinology 87(2), 286-93

Elvin, J.A., Clark, A.T., Wang, P., Wolfman, N.M., and Matzuk, M.M. (1999) Paracrine actions of growth differentiation factor-9 in the mammalian ovary. Mol Endocrinol 13(6), 1035-48

Eppig, J.J. (1979) FSH stimulates hyaluronic acid synthesis by oocyte-cumulus cell complexes from mouse preovulatory follicles. Nature 281(5731), 483-4 
Eppig, J.J. (2001) Oocyte control of ovarian follicular development and function in mammals. Reproduction 122(6), 829-38

Eppig, J.J., Chesnel, F., Hirao, Y., O'Brien, M.J., Pendola, F.L., Watanabe, S., and Wigglesworth, K. (1997a) Oocyte control of granulosa cell development: how and why. Hum Reprod 12(11 Suppl), 127-32

Eppig, J.J., Pendola, F.L., Wigglesworth, K., and Pendola, J.K. (2005) Mouse oocytes regulate metabolic cooperativity between granulosa cells and oocytes: amino acid transport. Biol Reprod 73(2), 351-7

Eppig, J.J., Peters, A.H., Telfer, E.E., and Wigglesworth, K. (1993) Production of cumulus expansion enabling factor by mouse oocytes grown in vitro: preliminary characterization of the factor. Mol Reprod Dev 34(4), 450-6

Eppig, J.J., Wigglesworth, K., Pendola, F., and Hirao, Y. (1997b) Murine oocytes suppress expression of luteinizing hormone receptor messenger ribonucleic acid by granulosa cells. Biol Reprod 56(4), 976-84

Eppig, J.J., Wigglesworth, K., and Pendola, F.L. (2002) The mammalian oocyte orchestrates the rate of ovarian follicular development. Proc Natl Acad Sci U S A 99(5), 2890-4

Esinler, I., Bayar, U., Bozdag, G., and Yarali, H. (2005) Outcome of intracytoplasmic sperm injection in patients with polycystic ovary syndrome or isolated polycystic ovaries. Fertil Steril 84(4), 932-7

Fabbri, R., Porcu, E., Marsella, T., Primavera, M.R., Seracchioli, R., Ciotti, P.M., Magrini, O., Venturoli, S., and Flamigni, C. (1998) Oocyte cryopreservation. Hum Reprod 13 Suppl 4, 98108

Fauser, B.C., and Devroey, P. (2003) Reproductive biology and IVF: ovarian stimulation and luteal phase consequences. Trends Endocrinol Metab 14(5), 236-42

Ferre, F. (1992) Quantitative or semi-quantitative PCR: reality versus myth. PCR Methods Appl 2(1), 1-9

Feuerstein, P., Cadoret, V., Dalbies-Tran, R., Guerif, F., Bidault, R., and Royere, D. (2007) Gene expression in human cumulus cells: one approach to oocyte competence. Hum Reprod 22(12), 3069-77

Fishel, S.B., Edwards, R.G., Purdy, J.M., Steptoe, P.C., Webster, J., Walters, E., Cohen, J., Fehilly, C., Hewitt, J., and Rowland, G. (1985) Implantation, abortion, and birth after in vitro 
fertilization using the natural menstrual cycle or follicular stimulation with clomiphene citrate and human menopausal gonadotropin. J In Vitro Fert Embryo Transf 2(3), 123-31

Forti, G., and Krausz, C. (1998) Clinical review 100: Evaluation and treatment of the infertile couple. J Clin Endocrinol Metab 83(12), 4177-88

Franks, S., Stark, J., and Hardy, K. (2008) Follicle dynamics and anovulation in polycystic ovary syndrome. Hum Reprod Update 14(4), 367-78

Frota, I.M., Leitao, C.C., Costa, J.J., Brito, I.R., van den Hurk, R., and Silva, J.R. (2011) Stability of housekeeping genes and expression of locally produced growth factors and hormone receptors in goat preantral follicles. Zygote 19(1), 71-83

Fu, J., Bian, L., Zhao, L., Dong, Z., Gao, X., Luan, H., Sun, Y., and Song, H. (2010) Identification of genes for normalization of quantitative real-time PCR data in ovarian tissues. Acta Biochim Biophys Sin (Shanghai) 42(8), 568-74

Fujiwara, H., Tatsumi, K., Kosaka, K., Sato, Y., Higuchi, T., Yoshioka, S., Maeda, M., Ueda, M., and Fujii, S. (2003) Human blastocysts and endometrial epithelial cells express activated leukocyte cell adhesion molecule (ALCAM/CD166). J Clin Endocrinol Metab 88(7), 343743

Fulop, C., Salustri, A., and Hascall, V.C. (1997) Coding sequence of a hyaluronan synthase homologue expressed during expansion of the mouse cumulus-oocyte complex. Arch Biochem Biophys 337(2), 261-6

Fulop, C., Szanto, S., Mukhopadhyay, D., Bardos, T., Kamath, R.V., Rugg, M.S., Day, A.J., Salustri, A., Hascall, V.C., Glant, T.T., and Mikecz, K. (2003) Impaired cumulus mucification and female sterility in tumor necrosis factor-induced protein-6 deficient mice. Development 130(10), 2253-61

Galloway, S.M., McNatty, K.P., Cambridge, L.M., Laitinen, M.P., Juengel, J.L., Jokiranta, T.S., McLaren, R.J., Luiro, K., Dodds, K.G., Montgomery, G.W., Beattie, A.E., Davis, G.H., and Ritvos, O. (2000) Mutations in an oocyte-derived growth factor gene (BMP15) cause increased ovulation rate and infertility in a dosage-sensitive manner. Nat Genet 25(3), 279-83

Gardner, D.K., and Sakkas, D. (2003) Assessment of embryo viability: the ability to select a single embryo for transfer--a review. Placenta 24 Suppl B, S5-12 
Gardner, D.K., Schoolcraft, W.B., Wagley, L., Schlenker, T., Stevens, J., and Hesla, J. (1998) A prospective randomized trial of blastocyst culture and transfer in in-vitro fertilization. Hum Reprod 13(12), 3434-40

Gebhardt, K.M., Feil, D.K., Dunning, K.R., Lane, M., and Russell, D.L. (2011) Human cumulus cell gene expression as a biomarker of pregnancy outcome after single embryo transfer. Fertil Steril 96(1), 47-52 e2

Gianaroli, L., Magli, M.C., Ferraretti, A.P., and Munne, S. (1999) Preimplantation diagnosis for aneuploidies in patients undergoing in vitro fertilization with a poor prognosis: identification of the categories for which it should be proposed. Fertil Steril 72(5), 837-44

Gilchrist, R.B., Lane, M., and Thompson, J.G. (2008) Oocyte-secreted factors: regulators of cumulus cell function and oocyte quality. Hum Reprod Update 14(2), 159-77

Gillett, W.R., Putt, T., and Farquhar, C.M. (2006) Prioritising for fertility treatments--the effect of excluding women with a high body mass index. BJOG 113(10), 1218-21

Gilula, N.B., Epstein, M.L., and Beers, W.H. (1978) Cell-to-cell communication and ovulation. A study of the cumulus-oocyte complex. J Cell Biol 78(1), 58-75

Goldzieher, J.W., and Green, J.A. (1962) The polycystic ovary. I. Clinical and histologic features. J Clin Endocrinol Metab 22, 325-38

Gonen, Y., Jacobson, W., and Casper, R.F. (1990) Gonadotropin suppression with oral contraceptives before in vitro fertilization. Fertil Steril 53(2), 282-7

Gonzalez-Fernandez, R., Pena, O., Hernandez, J., Martin-Vasallo, P., Palumbo, A., and Avila, J. (2010) FSH receptor, KL1/2, P450, and PAPP genes in granulosa-lutein cells from in vitro fertilization patients show a different expression pattern depending on the infertility diagnosis. Fertil Steril 94(1), 99-104

Goold, I., and Savulescu, J. (2009) In favour of freezing eggs for non-medical reasons. Bioethics 23(1), 47-58

Gougeon, A. (1989) Some aspects of the dynamics of ovarian follicular growth in the human. Acta Eur Fertil 20(4), 185-92

Gougeon, A. (1998) Ovarian follicular growth in humans: ovarian ageing and population of growing follicles. Maturitas 30(2), 137-42

Gougeon, A. (2010) Human ovarian follicular development: from activation of resting follicles to preovulatory maturation. Ann Endocrinol (Paris) 71(3), 132-43 
Gougeon, A., and Chainy, G.B. (1987) Morphometric studies of small follicles in ovaries of women at different ages. J Reprod Fertil 81(2), 433-42

Greenseid, K., Jindal, S., Hurwitz, J., Santoro, N., and Pal, L. (2011) Differential granulosa cell gene expression in young women with diminished ovarian reserve. Reprod Sci 18(9), 892-9

Griswold, M.D., Heckert, L., and Linder, C. (1995) The molecular biology of the FSH receptor. $J$ Steroid Biochem Mol Biol 53(1-6), 215-8

Grondahl, M.L., Yding Andersen, C., Bogstad, J., Nielsen, F.C., Meinertz, H., and Borup, R. (2010) Gene expression profiles of single human mature oocytes in relation to age. Hum Reprod 25(4), 957-68

Groome, N.P., Illingworth, P.J., O'Brien, M., Pai, R., Rodger, F.E., Mather, J.P., and McNeilly, A.S. (1996) Measurement of dimeric inhibin B throughout the human menstrual cycle. J Clin Endocrinol Metab 81(4), 1401-5

Gudnason, H., Dufva, M., Bang, D.D., and Wolff, A. (2007) Comparison of multiple DNA dyes for real-time PCR: effects of dye concentration and sequence composition on DNA amplification and melting temperature. Nucleic Acids Res 35(19), e127

Gui, L.M., and Joyce, I.M. (2005) RNA interference evidence that growth differentiation factor-9 mediates oocyte regulation of cumulus expansion in mice. Biol Reprod 72(1), 195-9

Guo, G., Huss, M., Tong, G.Q., Wang, C., Li Sun, L., Clarke, N.D., and Robson, P. (2010) Resolution of cell fate decisions revealed by single-cell gene expression analysis from zygote to blastocyst. Dev Cell 18(4), 675-85

Hadorn, D.C., and Holmes, A.C. (1997) The New Zealand priority criteria project. Part 1: Overview. BMJ 314(7074), 131-4

Hamel, M., Dufort, I., Robert, C., Gravel, C., Leveille, M.C., Leader, A., and Sirard, M.A. (2008) Identification of differentially expressed markers in human follicular cells associated with competent oocytes. Hum Reprod 23(5), 1118-27

Hasegawa, J., Yanaihara, A., Iwasaki, S., Otsuka, Y., Negishi, M., Akahane, T., and Okai, T. (2005) Reduction of progesterone receptor expression in human cumulus cells at the time of oocyte collection during IVF is associated with good embryo quality. Hum Reprod 20(8), 2194-200

Hernandez-Gonzalez, I., Gonzalez-Robayna, I., Shimada, M., Wayne, C.M., Ochsner, S.A., White, L., and Richards, J.S. (2006) Gene expression profiles of cumulus cell oocyte 
complexes during ovulation reveal cumulus cells express neuronal and immune-related genes: does this expand their role in the ovulation process? Mol Endocrinol 20(6), 1300-21

Hodgen, G.D. (1991) GnRH analogs in reproductive medicine. Keio J Med 40(1), 25-32

Hohmann, F.P., Laven, J.S., de Jong, F.H., and Fauser, B.C. (2005) Relationship between inhibin $\mathrm{A}$ and $\mathrm{B}$, estradiol and follicle growth dynamics during ovarian stimulation in normoovulatory women. Eur J Endocrinol 152(3), 395-401

Holte, J., Bergh, T., Gennarelli, G., and Wide, L. (1994) The independent effects of polycystic ovary syndrome and obesity on serum concentrations of gonadotrophins and sex steroids in premenopausal women. Clin Endocrinol (Oxf) 41(4), 473-81

Host, E., Gabrielsen, A., Lindenberg, S., and Smidt-Jensen, S. (2002) Apoptosis in human cumulus cells in relation to zona pellucida thickness variation, maturation stage, and cleavage of the corresponding oocyte after intracytoplasmic sperm injection. Fertil Steril 77(3), 511-5

Hudlow, W.R., Chong, M.D., Swango, K.L., Timken, M.D., and Buoncristiani, M.R. (2008) A quadruplex real-time qPCR assay for the simultaneous assessment of total human DNA, human male DNA, DNA degradation and the presence of PCR inhibitors in forensic samples: a diagnostic tool for STR typing. Forensic Sci Int Genet 2(2), 108-25

Huggett, J., Dheda, K., Bustin, S., and Zumla, A. (2005) Real-time RT-PCR normalisation; strategies and considerations. Genes Immun 6(4), 279-84

Huirne, J.A., Homburg, R., and Lambalk, C.B. (2007) Are GnRH antagonists comparable to agonists for use in IVF? Hum Reprod 22(11), 2805-13

Huirne, J.A., Lambalk, C.B., van Loenen, A.C., Schats, R., Hompes, P.G., Fauser, B.C., and Macklon, N.S. (2004) Contemporary pharmacological manipulation in assisted reproduction. Drugs 64(3), 297-322

Huirne, J.A., van Loenen, A.C., Donnez, J., Pirard, C., Homburg, R., Schats, R., McDonnell, J., and Lambalk, C.B. (2006) Effect of an oral contraceptive pill on follicular development in IVF/ICSI patients receiving a GnRH antagonist: a randomized study. Reprod Biomed Online 13(2), 235-45

Hussein, T.S., Froiland, D.A., Amato, F., Thompson, J.G., and Gilchrist, R.B. (2005) Oocytes prevent cumulus cell apoptosis by maintaining a morphogenic paracrine gradient of bone morphogenetic proteins. J Cell Sci 118(Pt 22), 5257-68 
Hussein, T.S., Thompson, J.G., and Gilchrist, R.B. (2006) Oocyte-secreted factors enhance oocyte developmental competence. Dev Biol 296(2), 514-21

Iolyeva, M., Karaman, S., Willrodt, A.H., Weingartner, S., Vigl, B., and Halin, C. (2012) Novel role for ALCAM in lymphatic network formation and function. FASEB $J$

Iwai, T., Nanbu, Y., Iwai, M., Taii, S., Fujii, S., and Mori, T. (1990) Immunohistochemical localization of oestrogen receptors and progesterone receptors in the human ovary throughout the menstrual cycle. Virchows Arch A Pathol Anat Histopathol 417(5), 369-75

Janssens, R.M., Lambalk, C.B., Vermeiden, J.P., Schats, R., Bernards, J.M., Rekers-Mombarg, L.T., and Schoemaker, J. (2000) Dose-finding study of triptorelin acetate for prevention of a premature LH surge in IVF: a prospective, randomized, double-blind, placebo-controlled study. Hum Reprod 15(11), 2333-40

Jindal, S., Greenseid, K., Berger, D., Santoro, N., and Pal, L. (2012) Impaired gremlin 1 (GREM1) expression in cumulus cells in young women with diminished ovarian reserve (DOR). J Assist Reprod Genet 29(2), 159-62

Josso, N. (1990) Anti-mullerian hormone: hormone or growth factor? Prog Growth Factor Res 2(3), 169-79

Joyce, I.M., Pendola, F.L., Wigglesworth, K., and Eppig, J.J. (1999) Oocyte regulation of kit ligand expression in mouse ovarian follicles. Dev Biol 214(2), 342-53

Juengel, J.L., Hudson, N.L., Berg, M., Hamel, K., Smith, P., Lawrence, S.B., Whiting, L., and McNatty, K.P. (2009) Effects of active immunization against growth differentiation factor 9 and/or bone morphogenetic protein 15 on ovarian function in cattle. Reproduction 138(1), 107-14

Juengel, J.L., Hudson, N.L., Heath, D.A., Smith, P., Reader, K.L., Lawrence, S.B., O'Connell, A.R., Laitinen, M.P., Cranfield, M., Groome, N.P., Ritvos, O., and McNatty, K.P. (2002) Growth differentiation factor 9 and bone morphogenetic protein 15 are essential for ovarian follicular development in sheep. Biol Reprod 67(6), 1777-89

Kaestner, K.H., Christy, R.J., and Lane, M.D. (1990) Mouse insulin-responsive glucose transporter gene: characterization of the gene and trans-activation by the CCAAT/enhancer binding protein. Proc Natl Acad Sci U S A 87(1), 251-5 
Kim, J., Sato, M., Li, Q., Lydon, J.P., Demayo, F.J., Bagchi, I.C., and Bagchi, M.K. (2008) Peroxisome proliferator-activated receptor gamma is a target of progesterone regulation in the preovulatory follicles and controls ovulation in mice. Mol Cell Biol 28(5), 1770-82

Kimura, N., Konno, Y., Miyoshi, K., Matsumoto, H., and Sato, E. (2002) Expression of hyaluronan synthases and CD44 messenger RNAs in porcine cumulus-oocyte complexes during in vitro maturation. Biol Reprod 66(3), 707-17

Kirby, T. (2010) Robert Edwards: Nobel Prize for father of in-vitro fertilisation. Lancet 376(9749), 1293

Klipstein, S., Regan, M., Ryley, D.A., Goldman, M.B., Alper, M.M., and Reindollar, R.H. (2005) One last chance for pregnancy: a review of 2,705 in vitro fertilization cycles initiated in women age 40 years and above. Fertil Steril 84(2), 435-45

Knight, P.G., and Glister, C. (2006) TGF-beta superfamily members and ovarian follicle development. Reproduction 132(2), 191-206

Knochenhauer, E.S., Key, T.J., Kahsar-Miller, M., Waggoner, W., Boots, L.R., and Azziz, R. (1998) Prevalence of the polycystic ovary syndrome in unselected black and white women of the southeastern United States: a prospective study. J Clin Endocrinol Metab 83(9), 3078-82

Kolle, S., Reese, S., and Kummer, W. (2010) New aspects of gamete transport, fertilization, and embryonic development in the oviduct gained by means of live cell imaging. Theriogenology 73(6), 786-95

Koppelkamm, A., Vennemann, B., Lutz-Bonengel, S., Fracasso, T., and Vennemann, M. (2011) RNA integrity in post-mortem samples: influencing parameters and implications on RTqPCR assays. Int J Legal Med 125(4), 573-80

Kufner, K., Tonne, M., and Barth, J. (2009) What is to be done with surplus embryos? Attitude formation with ambivalence in German fertility patients. Reprod Biomed Online 18 Suppl 1, 68-77

Kuo, H.C., Wang, T.Y., Chen, P.P., Chen, Y.M., Chuang, H.C., and Chen, T.Y. (2011) Real-time quantitative PCR assay for monitoring of nervous necrosis virus infection in grouper aquaculture. J Clin Microbiol 49(3), 1090-6

Kuroda, K., Utani, A., Hamasaki, Y., and Shinkai, H. (2001) Up-regulation of putative hyaluronan synthase mRNA by basic fibroblast growth factor and insulin-like growth factor-1 in human skin fibroblasts. J Dermatol Sci 26(2), 156-60 
Land, J.A., and Evers, J.L. (2003) Risks and complications in assisted reproduction techniques: Report of an ESHRE consensus meeting. Hum Reprod 18(2), 455-7

Larsen, W.J., Wert, S.E., and Brunner, G.D. (1986) A dramatic loss of cumulus cell gap junctions is correlated with germinal vesicle breakdown in rat oocytes. Dev Biol 113(2), 517-21

Lass, A., Croucher, C., Duffy, S., Dawson, K., Margara, R., and Winston, R.M. (1998) One thousand initiated cycles of in vitro fertilization in women $>$ or $=40$ years of age. Fertil Steril 70(6), 1030-4

Lebaron, R.G. (1996) Versican. Perspect Dev Neurobiol 3(4), 261-71

Le Bouc, Y., Rossignol, S., Azzi, S., Steunou, V., Netchine, I., and Gicquel, C. (2010) Epigenetics, genomic imprinting and assisted reproductive technology. Ann Endocrinol (Paris) 71(3), 237-8

Lee, K.S., Joo, B.S., Na, Y.J., Yoon, M.S., Choi, O.H., and Kim, W.W. (2001) Cumulus cells apoptosis as an indicator to predict the quality of oocytes and the outcome of IVF-ET. $J$ Assist Reprod Genet 18(9), 490-8

Legro, R.S., Kunselman, A.R., Dodson, W.C., and Dunaif, A. (1999) Prevalence and predictors of risk for type 2 diabetes mellitus and impaired glucose tolerance in polycystic ovary syndrome: a prospective, controlled study in 254 affected women. J Clin Endocrinol Metab 84(1), 165-9

Levi Setti, P.E., Albani, E., Cesana, A., Novara, P.V., Zannoni, E., Baggiani, A.M., Morenghi, E., Arfuso, V., and Scaravelli, G. (2011) Italian Constitutional Court modifications of a restrictive assisted reproduction technology law significantly improve pregnancy rate. Hum Reprod 26(2), 376-81

Li, Q., McKenzie, L.J., and Matzuk, M.M. (2008) Revisiting oocyte-somatic cell interactions: in search of novel intrafollicular predictors and regulators of oocyte developmental competence. Mol Hum Reprod 14(12), 673-8

Li, R., Norman, R.J., Armstrong, D.T., and Gilchrist, R.B. (2000) Oocyte-secreted factor(s) determine functional differences between bovine mural granulosa cells and cumulus cells. Biol Reprod 63(3), 839-45

Livak, K.J., and Schmittgen, T.D. (2001) Analysis of relative gene expression data using realtime quantitative PCR and the 2(-Delta Delta C(T)) Method. Methods 25(4), 402-8 
Ludwig, M., Finas, D.F., al-Hasani, S., Diedrich, K., and Ortmann, O. (1999) Oocyte quality and treatment outcome in intracytoplasmic sperm injection cycles of polycystic ovarian syndrome patients. Hum Reprod 14(2), 354-8

Lundy, T., Smith, P., O'Connell, A., Hudson, N.L., and McNatty, K.P. (1999) Populations of granulosa cells in small follicles of the sheep ovary. J Reprod Fertil 115(2), 251-62

Macklon, N.S., and Fauser, B.C. (1998) Follicle development during the normal menstrual cycle. Maturitas 30(2), 181-8

Maheshwari, A., Gibreel, A., Siristatidis, C.S., and Bhattacharya, S. (2011) Gonadotrophinreleasing hormone agonist protocols for pituitary suppression in assisted reproduction. Cochrane Database Syst Rev(8), CD006919

Maltaris, T., Beckmann, M.W., and Dittrich, R. (2009) Review. Fertility preservation for young female cancer patients. In Vivo 23(1), 123-30

Mandelbaum, J. (2000) Oocytes. Hum Reprod 15 Suppl 4, 11-8

McIntosh, C.J., Lawrence, S., Smith, P., Juengel, J.L., and McNatty, K.P. (2012) Active immunization against the proregions of GDF9 or BMP15 alters ovulation rate and litter size in mice. Reproduction 143(2), 195-201

McKenzie, L.J., Pangas, S.A., Carson, S.A., Kovanci, E., Cisneros, P., Buster, J.E., Amato, P., and Matzuk, M.M. (2004) Human cumulus granulosa cell gene expression: a predictor of fertilization and embryo selection in women undergoing IVF. Hum Reprod 19(12), 2869-74

McNatty, K.P. (1978) Cyclic changes in antral fluid hormone concentrations in humans. Clin Endocrinol Metab 7(3), 577-600

McNatty, K.P. (1978c) Follicular fluid. In The Vertebrate Ovary, 215-259. Ed. RE Jones. London: Plenum Press.

McNatty, K.P., and Baird, D.T. (1978) Relationship between follicle-stimulating hormone, androstenedione and oestradiol in human follicular fluid. J Endocrinol 76(3), 527-31

McNatty, K.P., Dobson, C., Gibb, M., Kieboom, L., and Thurley, D.C. (1981a) Accumulation of luteinizing hormone, oestradiol and androstenedione by sheep ovarian follicles in vivo. $J$ Endocrinol 91(1), 99-109

McNatty, K.P., Fidler, A.E., Juengel, J.L., Quirke, L.D., Smith, P.R., Heath, D.A., Lundy, T., O'Connell, A., and Tisdall, D.J. (2000) Growth and paracrine factors regulating follicular formation and cellular function. Mol Cell Endocrinol 163(1-2), 11-20 
McNatty, K.P., Gibb, M., Dobson, C., Thurley, D.C., and Findlay, J.K. (1981b) Changes in the concentration of gonadotropic and steroidal hormones in the antral fluid of ovarian follicle throughout the oestrous cycle of the sheep. Aust J Biol Sci 34(1), 67-80

McNatty, K.P., Heath, D.A., Henderson, K.M., Lun, S., Hurst, P.R., Ellis, L.M., Montgomery, G.W., Morrison, L., and Thurley, D.C. (1984) Some aspects of thecal and granulosa cell function during follicular development in the bovine ovary. $J$ Reprod Fertil 72(1), 39-53

McNatty, K.P., Heath, D.A., Hudson, N., and Clarke, I.J. (1990) Effect of long-term hypophysectomy on ovarian follicle populations and gonadotrophin-induced adenosine cyclic 3',5'-monophosphate output by follicles from Booroola ewes with or without the F gene. $J$ Reprod Fertil 90(2), 515-22

McNatty, K.P., Heath, D.A., Hudson, N.L., Reader, K.L., Quirke, L., Lun, S., and Juengel, J.L. (2010) The conflict between hierarchical ovarian follicular development and superovulation treatment. Reproduction 140(2), 287-94

McNatty, K.P., Heath, D.A., Lundy, T., Fidler, A.E., Quirke, L., O'Connell, A., Smith, P., Groome, N., and Tisdall, D.J. (1999) Control of early ovarian follicular development. $J$ Reprod Fertil Suppl 54, 3-16

McNatty, K.P., Hillier, S.G., van den Boogaard, A.M., Trimbos-Kemper, T.C., Reichert, L.E., Jr., and van Hall, E.V. (1983) Follicular development during the luteal phase of the human menstrual cycle. J Clin Endocrinol Metab 56(5), 1022-31

McNatty, K.P., Juengel, J.L., Wilson, T., Galloway, S.M., Davis, G.H., Hudson, N.L., Moeller, C.L., Cranfield, M., Reader, K.L., Laitinen, M.P., Groome, N.P., Sawyer, H.R., and Ritvos, O. (2003) Oocyte-derived growth factors and ovulation rate in sheep. Reprod Suppl 61, 33951

McNatty, K.P., Lawrence, S., Groome, N.P., Meerasahib, M.F., Hudson, N.L., Whiting, L., Heath, D.A., and Juengel, J.L. (2006) Meat and Livestock Association Plenary Lecture 2005. Oocyte signalling molecules and their effects on reproduction in ruminants. Reprod Fertil Dev 18(4), 403-12

McNatty, K.P., Moore, L.G., Hudson, N.L., Quirke, L.D., Lawrence, S.B., Reader, K., Hanrahan, J.P., Smith, P., Groome, N.P., Laitinen, M., Ritvos, O., and Juengel, J.L. (2004) The oocyte and its role in regulating ovulation rate: a new paradigm in reproductive biology. Reproduction 128(4), 379-86 
Meldrum, D.R., Wisot, A., Hamilton, F., Gutlay, A.L., Kempton, W.F., and Huynh, D. (1989) Routine pituitary suppression with leuprolide before ovarian stimulation for oocyte retrieval. Fertil Steril 51(3), 455-9

Mellembakken, J.R., Berga, S.L., Kilen, M., Tanbo, T.G., Abyholm, T., and Fedorcsak, P. (2011) Sustained fertility from 22 to 41 years of age in women with polycystic ovarian syndrome. Hum Reprod 26(9), 2499-504

Merton, J.S., de Roos, A.P., Mullaart, E., de Ruigh, L., Kaal, L., Vos, P.L., and Dieleman, S.J. (2003) Factors affecting oocyte quality and quantity in commercial application of embryo technologies in the cattle breeding industry. Theriogenology 59(2), 651-74

Moffatt, O., Drury, S., Tomlinson, M., Afnan, M., and Sakkas, D. (2002) The apoptotic profile of human cumulus cells changes with patient age and after exposure to sperm but not in relation to oocyte maturity. Fertil Steril 77(5), 1006-11

Mohler-Kuo, M., Zellweger, U., Duran, A., Hohl, M.K., Gutzwiller, F., and Mutsch, M. (2009) Attitudes of couples towards the destination of surplus embryos: results among couples with cryopreserved embryos in Switzerland. Hum Reprod 24(8), 1930-8

Muasher, S.J. (1992) Use of gonadotrophin-releasing hormone agonists in controlled ovarian hyperstimulation for in vitro fertilization. Clin Ther 14 Suppl A, 74-86

Murphy, L.D., Herzog, C.E., Rudick, J.B., Fojo, A.T., and Bates, S.E. (1990) Use of the polymerase chain reaction in the quantitation of mdr-1 gene expression. Biochemistry 29(45), $10351-6$

Naso, M.F., Zimmermann, D.R., and Iozzo, R.V. (1994) Characterization of the complete genomic structure of the human versican gene and functional analysis of its promoter. $J$ Biol Chem 269(52), 32999-3008

Nel-Themaat, L., and Nagy, Z.P. (2011) A review of the promises and pitfalls of oocyte and embryo metabolomics. Placenta 32 Suppl 3, S257-63

Nelissen, E.C., Van Montfoort, A.P., Coonen, E., Derhaag, J.G., Geraedts, J.P., Smits, L.J., Land, J.A., Evers, J.L., and Dumoulin, J.C. (2012) Further evidence that culture media affect perinatal outcome: findings after transfer of fresh and cryopreserved embryos. Hum Reprod 27(7), 1966-76 
Ng, S.T., Chang, T.H., and Wu, T.C. (1999) Prediction of the rates of fertilization, cleavage, and pregnancy success by cumulus-coronal morphology in an in vitro fertilization program. Fertil Steril 72(3), 412-7

Nishimoto, H., Matsutani, R., Yamamoto, S., Takahashi, T., Hayashi, K.G., Miyamoto, A., Hamano, S., and Tetsuka, M. (2006) Gene expression of glucose transporter (GLUT) 1, 3 and 4 in bovine follicle and corpus luteum. J Endocrinol 188(1), 111-9

Noonan, K.E., Beck, C., Holzmayer, T.A., Chin, J.E., Wunder, J.S., Andrulis, I.L., Gazdar, A.F., Willman, C.L., Griffith, B., Von Hoff, D.D., and et al. (1990) Quantitative analysis of MDR1 (multidrug resistance) gene expression in human tumors by polymerase chain reaction. Proc Natl Acad Sci U S A 87(18), 7160-4

Noyes, N., Boldt, J., and Nagy, Z.P. (2010) Oocyte cryopreservation: is it time to remove its experimental label? J Assist Reprod Genet 27(2-3), 69-74

Ochsner, S.A., Russell, D.L., Day, A.J., Breyer, R.M., and Richards, J.S. (2003) Decreased expression of tumor necrosis factor-alpha-stimulated gene 6 in cumulus cells of the cyclooxygenase-2 and EP2 null mice. Endocrinology 144(3), 1008-19

Ohno, S., Klinger, H.P., and Atkin, N.B. (1962) Human oogenesis. Cytogenetics 1, 42-51

Oktem, O., and Oktay, K. (2008) The ovary: anatomy and function throughout human life. Ann N Y Acad Sci 1127, 1-9

Oktem, O., and Urman, B. (2010) Understanding follicle growth in vivo. Hum Reprod 25(12), 2944-54

Orvieto, R., Meltcer, S., Nahum, R., Rabinson, J., Anteby, E.Y., and Ashkenazi, J. (2009) The influence of body mass index on in vitro fertilization outcome. Int J Gynaecol Obstet 104(1), 53-5

Otsuka, F., Moore, R.K., Wang, X., Sharma, S., Miyoshi, T., and Shimasaki, S. (2005) Essential role of the oocyte in estrogen amplification of follicle-stimulating hormone signaling in granulosa cells. Endocrinology 146(8), 3362-7

Packer, A.I., Hsu, Y.C., Besmer, P., and Bachvarova, R.F. (1994) The ligand of the c-kit receptor promotes oocyte growth. Dev Biol 161(1), 194-205

Palermo, R. (2007) Differential actions of FSH and LH during folliculogenesis. Reprod Biomed Online 15(3), 326-37 
Park, O.K., and Mayo, K.E. (1991) Transient expression of progesterone receptor messenger RNA in ovarian granulosa cells after the preovulatory luteinizing hormone surge. Mol Endocrinol 5(7), 967-78

Patrizio, P., and Sakkas, D. (2009) From oocyte to baby: a clinical evaluation of the biological efficiency of in vitro fertilization. Fertil Steril 91(4), 1061-6

Paynter, S.J. (2000) Current status of the cryopreservation of human unfertilized oocytes. Hum Reprod Update 6(5), 449-56

Pelinck, M.J., Hoek, A., Simons, A.H., and Heineman, M.J. (2002) Efficacy of natural cycle IVF: a review of the literature. Hum Reprod Update 8(2), 129-39

Peters, H. (1976) Intrauterine gonadal development. Fertil Steril 27(5), 493-500

Peters, H and McNatty K.P. (1980) 'The Ovary' The Female Germ Cells and Oogenesis, Granada Publishing Ltd.

Peters, H., Byskov, A.G., Himelstein-Braw, R., and Faber, M. (1975) Follicular growth: the basic event in the mouse and human ovary. J Reprod Fertil 45(3), 559-66

Petersen, C.G., Oliveira, J.B., Mauri, A.L., Massaro, F.C., Baruffi, R.L., Pontes, A., and Franco, J.G., Jr. (2009) Relationship between visualization of meiotic spindle in human oocytes and ICSI outcomes: a meta-analysis. Reprod Biomed Online 18(2), 235-43

Phillips, S.J., Kadoch, I.J., Lapensee, L., Couturier, B., Hemmings, R., and Bissonnette, F. (2007) Controlled natural cycle IVF: experience in a world of stimulation. Reprod Biomed Online 14(3), 356-9

Pienimaki, J.P., Rilla, K., Fulop, C., Sironen, R.K., Karvinen, S., Pasonen, S., Lammi, M.J., Tammi, R., Hascall, V.C., and Tammi, M.I. (2001) Epidermal growth factor activates hyaluronan synthase 2 in epidermal keratinocytes and increases pericellular and intracellular hyaluronan. J Biol Chem 276(23), 20428-35

Pigny, P., Merlen, E., Robert, Y., Cortet-Rudelli, C., Decanter, C., Jonard, S., and Dewailly, D. (2003) Elevated serum level of anti-mullerian hormone in patients with polycystic ovary syndrome: relationship to the ovarian follicle excess and to the follicular arrest. J Clin Endocrinol Metab 88(12), 5957-62

Pinborg, A., Gaarslev, C., Hougaard, C.O., Nyboe Andersen, A., Andersen, P.K., Boivin, J., and Schmidt, L. (2011) Influence of female bodyweight on IVF outcome: a longitudinal multicentre cohort study of 487 infertile couples. Reprod Biomed Online 23(4), 490-9 
Princivalle, M., Hasan, S., Hosseini, G., and de Agostini, A.I. (2001) Anticoagulant heparan sulfate proteoglycans expression in the rat ovary peaks in preovulatory granulosa cells. Glycobiology 11(3), 183-94

Quackenbush, J. (2002) Microarray data normalization and transformation. Nat Genet 32 Suppl, 496-501

Rabinovici, J., and Jaffe, R.B. (1990) Development and regulation of growth and differentiated function in human and subhuman primate fetal gonads. Endocr Rev 11(4), 532-57

Rai, A.J., Kamath, R.M., Gerald, W., and Fleisher, M. (2009) Analytical validation of the GeXP analyzer and design of a workflow for cancer-biomarker discovery using multiplexed geneexpression profiling. Anal Bioanal Chem 393(5), 1505-11

Rattanachaiyanont, M., Leader, A., and Leveille, M.C. (1999) Lack of correlation between oocyte-corona-cumulus complex morphology and nuclear maturity of oocytes collected in stimulated cycles for intracytoplasmic sperm injection. Fertil Steril 71(5), 937-40

Reindollar, R.H., Regan, M.M., Neumann, P.J., Levine, B.S., Thornton, K.L., Alper, M.M., and Goldman, M.B. (2010) A randomized clinical trial to evaluate optimal treatment for unexplained infertility: the fast track and standard treatment (FASTT) trial. Fertil Steril 94(3), 888-99

Rice, S., Christoforidis, N., Gadd, C., Nikolaou, D., Seyani, L., Donaldson, A., Margara, R., Hardy, K., and Franks, S. (2005) Impaired insulin-dependent glucose metabolism in granulosa-lutein cells from anovulatory women with polycystic ovaries. Hum Reprod 20(2), 373-81

Rice, S., Pellatt, L.J., Bryan, S.J., Whitehead, S.A., and Mason, H.D. (2011) Action of metformin on the insulin-signaling pathway and on glucose transport in human granulosa cells. J Clin Endocrinol Metab 96(3), E427-35

Rienzi, L., Ubaldi, F., Martinez, F., Iacobelli, M., Minasi, M.G., Ferrero, S., Tesarik, J., and Greco, E. (2003) Relationship between meiotic spindle location with regard to the polar body position and oocyte developmental potential after ICSI. Hum Reprod 18(6), 1289-93

Rienzi, L., Vajta, G., and Ubaldi, F. (2011) Predictive value of oocyte morphology in human IVF: a systematic review of the literature. Hum Reprod Update 17(1), 34-45

Roberts, R., Stark, J., Iatropoulou, A., Becker, D.L., Franks, S., and Hardy, K. (2004) Energy substrate metabolism of mouse cumulus-oocyte complexes: response to follicle-stimulating 
hormone is mediated by the phosphatidylinositol 3-kinase pathway and is associated with oocyte maturation. Biol Reprod 71(1), 199-209

Robker, R.L., Akison, L.K., and Russell, D.L. (2009) Control of oocyte release by progesterone receptor-regulated gene expression. Nucl Recept Signal 7, e012

Romero, S., Sanchez, F., Adriaenssens, T., and Smitz, J. (2011) Mouse cumulus-oocyte complexes from in vitro-cultured preantral follicles suggest an anti-luteinizing role for the EGF cascade in the cumulus cells. Biol Reprod 84(6), 1164-70

Rosenbaum, D., Haber, R.S., and Dunaif, A. (1993) Insulin resistance in polycystic ovary syndrome: decreased expression of GLUT-4 glucose transporters in adipocytes. Am J Physiol 264(2 Pt 1), E197-202

Ross, G.T., Cargille, C.M., Lipsett, M.B., Rayford, P.L., Marshall, J.R., Strott, C.A., and Rodbard, D. (1970) Pituitary and gonadal hormones in women during spontaneous and induced ovulatory cycles. Recent Prog Horm Res 26, 1-62

Rotterdam ESHRE/ASRM PCOS consensus (2004) Revised 2003 consensus on diagnostic criteria and long-term health risks related to polycystic ovary syndrome (PCOS). Hum Reprod 19(1), 41-7

Russell, D.L., Ochsner, S.A., Hsieh, M., Mulders, S., and Richards, J.S. (2003) Hormoneregulated expression and localization of versican in the rodent ovary. Endocrinology 144(3), 1020-31

Russell, D.L., and Robker, R.L. (2007) Molecular mechanisms of ovulation: co-ordination through the cumulus complex. Hum Reprod Update 13(3), 289-312

Sagle, M., Bishop, K., Ridley, N., Alexander, F.M., Michel, M., Bonney, R.C., Beard, R.W., and Franks, S. (1988) Recurrent early miscarriage and polycystic ovaries. BMJ 297(6655), 1027-8

Sahu, B., Ozturk, O., Ranierri, M., and Serhal, P. (2008) Comparison of oocyte quality and intracytoplasmic sperm injection outcome in women with isolated polycystic ovaries or polycystic ovarian syndrome. Arch Gynecol Obstet 277(3), 239-44

Sakkas, D., Shoukir, Y., Chardonnens, D., Bianchi, P.G., and Campana, A. (1998) Early cleavage of human embryos to the two-cell stage after intracytoplasmic sperm injection as an indicator of embryo viability. Hum Reprod 13(1), 182-7

Saldeen, P., Kallen, K., and Sundstrom, P. (2007) The probability of successful IVF outcome after poor ovarian response. Acta Obstet Gynecol Scand 86(4), 457-61 
Salustri, A., Camaioni, A., Di Giacomo, M., Fulop, C., and Hascall, V.C. (1999) Hyaluronan and proteoglycans in ovarian follicles. Hum Reprod Update 5(4), 293-301

Salustri, A., Garlanda, C., Hirsch, E., De Acetis, M., Maccagno, A., Bottazzi, B., Doni, A., Bastone, A., Mantovani, G., Beck Peccoz, P., Salvatori, G., Mahoney, D.J., Day, A.J., Siracusa, G., Romani, L., and Mantovani, A. (2004) PTX3 plays a key role in the organization of the cumulus oophorus extracellular matrix and in in vivo fertilization. Development 131(7), 1577-86

Salustri, A., Yanagishita, M., and Hascall, V.C. (1989) Synthesis and accumulation of hyaluronic acid and proteoglycans in the mouse cumulus cell-oocyte complex during follicle-stimulating hormone-induced mucification. J Biol Chem 264(23), 13840-7

Sanders, A.J., Jiang, D.G., Jiang, W.G., Harding, K.G., and Patel, G.K. (2011) Activated leukocyte cell adhesion molecule impacts on clinical wound healing and inhibits $\mathrm{HaCaT}$ migration. Int Wound J 8(5), 500-7

Scaramuzzi, R.J., Adams, N.R., Baird, D.T., Campbell, B.K., Downing, J.A., Findlay, J.K., Henderson, K.M., Martin, G.B., McNatty, K.P., McNeilly, A.S., and et al. (1993) A model for follicle selection and the determination of ovulation rate in the ewe. Reprod Fertil Dev 5(5), $459-78$

Scaramuzzi, R.J., Baird, D.T., Campbell, B.K., Driancourt, M.A., Dupont, J., Fortune, J.E., Gilchrist, R.B., Martin, G.B., McNatty, K.P., McNeilly, A.S., Monget, P., Monniaux, D., Vinoles, C., and Webb, R. (2011) Regulation of folliculogenesis and the determination of ovulation rate in ruminants. Reprod Fertil Dev 23(3), 444-67

Schmidt, K.L., Kryger-Baggesen, N., Byskov, A.G., and Andersen, C.Y. (2005) Anti-Mullerian hormone initiates growth of human primordial follicles in vitro. Mol Cell Endocrinol 234(12), 87-93

Schoenfelder, M., and Einspanier, R. (2003) Expression of hyaluronan synthases and corresponding hyaluronan receptors is differentially regulated during oocyte maturation in cattle. Biol Reprod 69(1), 269-77

Schotsmans, P.T. (1998) In vitro fertilisation: the ethics of illicitness? A personalist Catholic approach. Eur J Obstet Gynecol Reprod Biol 81(2), 235-41 
Scott, R.T., and Navot, D. (1994) Enhancement of ovarian responsiveness with microdoses of gonadotropin-releasing hormone agonist during ovulation induction for in vitro fertilization. Fertil Steril 61(5), 880-5

Scott, R.T., Toner, J.P., Muasher, S.J., Oehninger, S., Robinson, S., and Rosenwaks, Z. (1989) Follicle-stimulating hormone levels on cycle day 3 are predictive of in vitro fertilization outcome. Fertil Steril 51(4), 651-4

Sharif, K., Elgendy, M., Lashen, H., and Afnan, M. (1998) Age and basal follicle stimulating hormone as predictors of in vitro fertilisation outcome. Br J Obstet Gynaecol 105(1), 107-12

Shoham, Z., Schacter, M., Loumaye, E., Weissman, A., MacNamee, M., and Insler, V. (1995) The luteinizing hormone surge--the final stage in ovulation induction: modern aspects of ovulation triggering. Fertil Steril 64(2), 237-51

Skinner, M.K. (2005) Regulation of primordial follicle assembly and development. Hum Reprod Update 11(5), 461-71

Smitz, J., Devroey, P., Camus, M., Deschacht, J., Khan, I., Staessen, C., Van Waesberghe, L., Wisanto, A., and Van Steirteghem, A.C. (1988) The luteal phase and early pregnancy after combined GnRH-agonist/HMG treatment for superovulation in IVF or GIFT. Hum Reprod 3(5), 585-90

Smulders, B., van Oirschot, S.M., Farquhar, C., Rombauts, L., and Kremer, J.A. (2010) Oral contraceptive pill, progestogen or estrogen pre-treatment for ovarian stimulation protocols for women undergoing assisted reproductive techniques. Cochrane Database Syst Rev(1), CD006109

Steptoe, P.C., and Edwards, R.G. (1978) Birth after the reimplantation of a human embryo. Lancet 2(8085), 366

Su, Y.Q., Denegre, J.M., Wigglesworth, K., Pendola, F.L., O'Brien, M.J., and Eppig, J.J. (2003) Oocyte-dependent activation of mitogen-activated protein kinase (ERK1/2) in cumulus cells is required for the maturation of the mouse oocyte-cumulus cell complex. Dev Biol 263(1), 126-38

Su, Y.Q., Wu, X., O'Brien, M.J., Pendola, F.L., Denegre, J.N., Matzuk, M.M., and Eppig, J.J. (2004) Synergistic roles of BMP15 and GDF9 in the development and function of the oocytecumulus cell complex in mice: genetic evidence for an oocyte-granulosa cell regulatory loop. Dev Biol 276(1), 64-73 
Sugiura, K., Pendola, F.L., and Eppig, J.J. (2005) Oocyte control of metabolic cooperativity between oocytes and companion granulosa cells: energy metabolism. Dev Biol 279(1), 20-30

Sugiura, K., Su, Y.Q., Diaz, F.J., Pangas, S.A., Sharma, S., Wigglesworth, K., O'Brien, M.J., Matzuk, M.M., Shimasaki, S., and Eppig, J.J. (2007) Oocyte-derived BMP15 and FGFs cooperate to promote glycolysis in cumulus cells. Development 134(14), 2593-603

Sugiura, K., Su, Y.Q., Li, Q., Wigglesworth, K., Matzuk, M.M., and Eppig, J.J. (2010) Estrogen promotes the development of mouse cumulus cells in coordination with oocyte-derived GDF9 and BMP15. Mol Endocrinol 24(12), 2303-14

Sutton, M.L., Gilchrist, R.B., and Thompson, J.G. (2003) Effects of in-vivo and in-vitro environments on the metabolism of the cumulus-oocyte complex and its influence on oocyte developmental capacity. Hum Reprod Update 9(1), 35-48

Swango, K.L., Hudlow, W.R., Timken, M.D., and Buoncristiani, M.R. (2007) Developmental validation of a multiplex qPCR assay for assessing the quantity and quality of nuclear DNA in forensic samples. Forensic Sci Int 170(1), 35-45

Swanton, A., Storey, L., McVeigh, E., and Child, T. (2010) IVF outcome in women with PCOS, PCO and normal ovarian morphology. Eur J Obstet Gynecol Reprod Biol 149(1), 68-71

Tarlatzis, B.C., Laufer, N., and Decherney, A.H. (1984) The use of ovarian ultrasonography in monitoring ovulation induction. J In Vitro Fert Embryo Transf 1(4), 226-32

Thomas, F.H., and Vanderhyden, B.C. (2006) Oocyte-granulosa cell interactions during mouse follicular development: regulation of kit ligand expression and its role in oocyte growth. Reprod Biol Endocrinol 4, 19

Toner, J.P., Philput, C.B., Jones, G.S., and Muasher, S.J. (1991) Basal follicle-stimulating hormone level is a better predictor of in vitro fertilization performance than age. Fertil Steril 55(4), 784-91

Toole, B.P. (1997) Hyaluronan in morphogenesis. J Intern Med 242(1), 35-40

Turley, E.A. (1992) Hyaluronan and cell locomotion. Cancer Metastasis Rev 11(1), 21-30

Ubaldi, F., Anniballo, R., Romano, S., Baroni, E., Albricci, L., Colamaria, S., Capalbo, A., Sapienza, F., Vajta, G., and Rienzi, L. (2010) Cumulative ongoing pregnancy rate achieved with oocyte vitrification and cleavage stage transfer without embryo selection in a standard infertility program. Hum Reprod 25(5), 1199-205 
University of Otago and Ministry of Health. (2011) A Focus on Nutrition: Key findings of the 2008/09 New Zealand Adult Nutrition Survey. Wellington: Ministry of Health.

van der Linden, M., Buckingham, K., Farquhar, C., Kremer, J.A., and Metwally, M. (2011) Luteal phase support for assisted reproduction cycles. Cochrane Database Syst Rev(10), CD009154

van Montfoort, A.P., Geraedts, J.P., Dumoulin, J.C., Stassen, A.P., Evers, J.L., and Ayoubi, T.A. (2008) Differential gene expression in cumulus cells as a prognostic indicator of embryo viability: a microarray analysis. Mol Hum Reprod 14(3), 157-68

van Rooij, I.A., Broekmans, F.J., te Velde, E.R., Fauser, B.C., Bancsi, L.F., de Jong, F.H., and Themmen, A.P. (2002) Serum anti-Mullerian hormone levels: a novel measure of ovarian reserve. Hum Reprod 17(12), 3065-71

van Wagenen, G. and Simpson, M.E. (1965) Embryology of the Ovary and Testis; Homo sapiens and Macaca mulatta. Yale University Press.

Vanderhyden, B.C., Cohen, J.N., and Morley, P. (1993) Mouse oocytes regulate granulosa cell steroidogenesis. Endocrinology 133(1), 423-6

Vanderhyden, B.C., and Tonary, A.M. (1995) Differential regulation of progesterone and estradiol production by mouse cumulus and mural granulosa cells by A factor(s) secreted by the oocyte. Biol Reprod 53(6), 1243-50

Vigier, B., Picard, J.Y., Tran, D., Legeai, L., and Josso, N. (1984) Production of anti-Mullerian hormone: another homology between Sertoli and granulosa cells. Endocrinology 114(4), $1315-20$

Wang, A.M., Doyle, M.V., and Mark, D.F. (1989) Quantitation of mRNA by the polymerase chain reaction. Proc Natl Acad Sci U S A 86(24), 9717-21

Wathlet, S., Adriaenssens, T., Segers, I., Verheyen, G., Van de Velde, H., Coucke, W., Ron El, R., Devroey, P., and Smitz, J. (2011) Cumulus cell gene expression predicts better cleavagestage embryo or blastocyst development and pregnancy for ICSI patients. Hum Reprod 26(5), $1035-51$

Watts, G. (2010) IVF pioneer Robert Edwards wins Nobel prize. BMJ 341, c5533

Weenen, C., Laven, J.S., Von Bergh, A.R., Cranfield, M., Groome, N.P., Visser, J.A., Kramer, P., Fauser, B.C., and Themmen, A.P. (2004) Anti-Mullerian hormone expression pattern in 
the human ovary: potential implications for initial and cyclic follicle recruitment. Mol Hum Reprod 10(2), 77-83

Weghofer, A., Munne, S., Chen, S., Barad, D., and Gleicher, N. (2007) Lack of association between polycystic ovary syndrome and embryonic aneuploidy. Fertil Steril 88(4), 900-5

Wells, D., and Delhanty, J.D. (2000) Comprehensive chromosomal analysis of human preimplantation embryos using whole genome amplification and single cell comparative genomic hybridization. Mol Hum Reprod 6(11), 1055-62

Willis, D.S., Watson, H., Mason, H.D., Galea, R., Brincat, M., and Franks, S. (1998) Premature response to luteinizing hormone of granulosa cells from anovulatory women with polycystic ovary syndrome: relevance to mechanism of anovulation. J Clin Endocrinol Metab 83(11), 3984-91

Wong, C.C., Loewke, K.E., Bossert, N.L., Behr, B., De Jonge, C.J., Baer, T.M., and Reijo Pera, R.A. (2010) Non-invasive imaging of human embryos before embryonic genome activation predicts development to the blastocyst stage. Nat Biotechnol 28(10), 1115-21

Wood, J.R., Dumesic, D.A., Abbott, D.H., and Strauss, J.F., 3rd (2007) Molecular abnormalities in oocytes from women with polycystic ovary syndrome revealed by microarray analysis. $J$ Clin Endocrinol Metab 92(2), 705-13

Yan, M., Wang, J., Wu, X., Hou, L., Kuang, H., and Wang, Y. (2009) Induction of insulin resistance by phosphatidylinositol-3-kinase inhibitor in porcine granulosa cells. Fertil Steril 92(6), 2119-21

Yang, M.Y., and Rajamahendran, R. (2000) Morphological and biochemical identification of apoptosis in small, medium, and large bovine follicles and the effects of follicle-stimulating hormone and insulin-like growth factor-I on spontaneous apoptosis in cultured bovine granulosa cells. Biol Reprod 62(5), 1209-17

Yen, S.S., Vela, P., and Rankin, J. (1970) Inappropriate secretion of follicle-stimulating hormone and luteinizing hormone in polycystic ovarian disease. J Clin Endocrinol Metab 30(4), 43542

Yeo, C.X., Gilchrist, R.B., Thompson, J.G., and Lane, M. (2008) Exogenous growth differentiation factor 9 in oocyte maturation media enhances subsequent embryo development and fetal viability in mice. Hum Reprod 23(1), 67-73 
Zander-Fox, D.L., Tremellen, K., and Lane, M. (2011) Single blastocyst embryo transfer maintains comparable pregnancy rates to double cleavage-stage embryo transfer but results in healthier pregnancy outcomes. Aust N Z J Obstet Gynaecol 51(5), 406-10

Zhang, M., Su, Y.Q., Sugiura, K., Wigglesworth, K., Xia, G., and Eppig, J.J. (2011) Estradiol promotes and maintains cumulus cell expression of natriuretic peptide receptor 2 (NPR2) and meiotic arrest in mouse oocytes in vitro. Endocrinology 152(11), 4377-85

Zhang, X., Jafari, N., Barnes, R.B., Confino, E., Milad, M., and Kazer, R.R. (2005) Studies of gene expression in human cumulus cells indicate pentraxin 3 as a possible marker for oocyte quality. Fertil Steril 83 Suppl 1, 1169-79

Ziebe, S., and Devroey, P. (2008) Assisted reproductive technologies are an integrated part of national strategies addressing demographic and reproductive challenges. Hum Reprod Update 14(6), 583-92 